\title{
ESTIMATION FOR CHANGE POINT OF DISCRETELY OBSERVED ERGODIC DIFFUSION PROCESSES
}

\author{
YOZO TONAKI, YUSUKE KAINO, AND MASAYUKI UCHIDA
}

\begin{abstract}
We treat the change point problem in ergodic diffusion processes from discrete observations. Tonaki et al. (2020) proposed adaptive tests for detecting changes in the diffusion and drift parameters in ergodic diffusion models. When any changes are detected by this method, the next question to be considered is where the change point is. Therefore, we propose the method to estimate the change point of the parameter for two cases: the case where there is a change in the diffusion parameter, and the case where there is no change in the diffusion parameter but a change in the drift parameter. Furthermore, we present rates of convergence and distributional results of the change point estimators. Some examples and simulation results are also given.
\end{abstract}

\section{INTRODUCTION}

We consider a $d$-dimensional diffusion process $\left\{X_{t}\right\}_{t \geq 0}$ satisfying the stochastic differential equation:

$$
\left\{\begin{array}{l}
\mathrm{d} X_{t}=b\left(X_{t}, \beta\right) \mathrm{d} t+a\left(X_{t}, \alpha\right) \mathrm{d} W_{t}, \\
X_{0}=x_{0},
\end{array}\right.
$$

where parameter space $\Theta=\Theta_{A} \times \Theta_{B}$, which is a compact convex subset of $\mathbb{R}^{p} \times \mathbb{R}^{q}, \theta=(\alpha, \beta) \in \Theta$ is an unknown parameter and $\left\{W_{t}\right\}_{t \geq 0}$ is a $d$-dimensional standard Wiener process. The diffusion coefficient $a: \mathbb{R}^{d} \times \Theta_{A} \longrightarrow \mathbb{R}^{d} \otimes \mathbb{R}^{d}$ and the drift coefficient $b: \mathbb{R}^{d} \times \Theta_{B} \longrightarrow \mathbb{R}^{d}$ are known except for the parameter $\theta$, and the true parameter $\theta^{*}=\left(\alpha^{*}, \beta^{*}\right)$ belongs to Int $\Theta$. We assume that the solution of (1.1) exists, and $P_{\theta}$ and $\mathbb{E}_{\theta}$ denote the law of the solution and the expectation with respect to $P_{\theta}$, respectively. Let $\left\{X_{t_{i}}\right\}_{i=0}^{n}$ be discrete observations, where $t_{i}=t_{i}^{n}=i h_{n}$, and $\left\{h_{n}\right\}$ is a positive sequence with $h=h_{n} \longrightarrow 0, T=n h \longrightarrow \infty$ and $n h^{2} \longrightarrow 0$ as $n \rightarrow \infty$.

We deal with the change point problem of parameters in diffusion process models. The change point problem consists of two parts: detection of the parameter change and estimation of the change point. For example, suppose that we obtain the paths shown in Figure 1. Given such data, the first thing we would consider is whether any changes occur in that path. The paths in Figure 1 clearly shows that some change occurs, but the paths in Figure 2 may be difficult to find a change. The first step of the change point problem is to investigate whether there is a change in the data or not, which is "detection of the parameter change". Suppose that we can detect a change in the paths in Figure 1 or 2 In Figure 1 we can visually see that the change occurs at $t=60$ in the left figure and at $t=40$ in the right figure, but the paths in Figure 2 are not even visually recognizable, so we have no idea where the change occurs. The goal of the change point problem is to investigate the change point when we see that there is a change in the data, which is "estimation of the change point".

The change point problem for diffusion processes from discrete observations has been studied by many researchers. For non-ergodic diffusion processes, see De Gregorio and Iacus (2008) and Iacus and Yoshida (2012). For ergodic diffusion processes, we can refer to Song and Lee (2009), Lee (2011), Negri and Nishiyama (2017), Song (2020), and Tonaki et al. (2020). De Gregorio and Iacus (2008) considered the detection of the parameter change in diffusion parameter and the estimation of the change point, and Iacus and Yoshida

(Y. Tonaki) Graduate School of Engineering Science, Osaka University, 1-3, Machikaneyama, Toyonaka, Osaka, 560-8531, JAPAN

(Y. Kaino) Graduate School of Engineering Science, Osaka University, 1-3, Machikaneyama, Toyonaka, Osaka, 560-8531, JAPAN

(M. Uchida) Graduate School of Engineering Science, and Center for Mathematical Modeling and Date Science, Osaka University, 1-3, Machikaneyama, Toyonaka, Osaka, 560-8531, Japan

Key words and phrases. adaptive tests, change point estimation, diffusion processes, discrete observations, test for parameter change. 

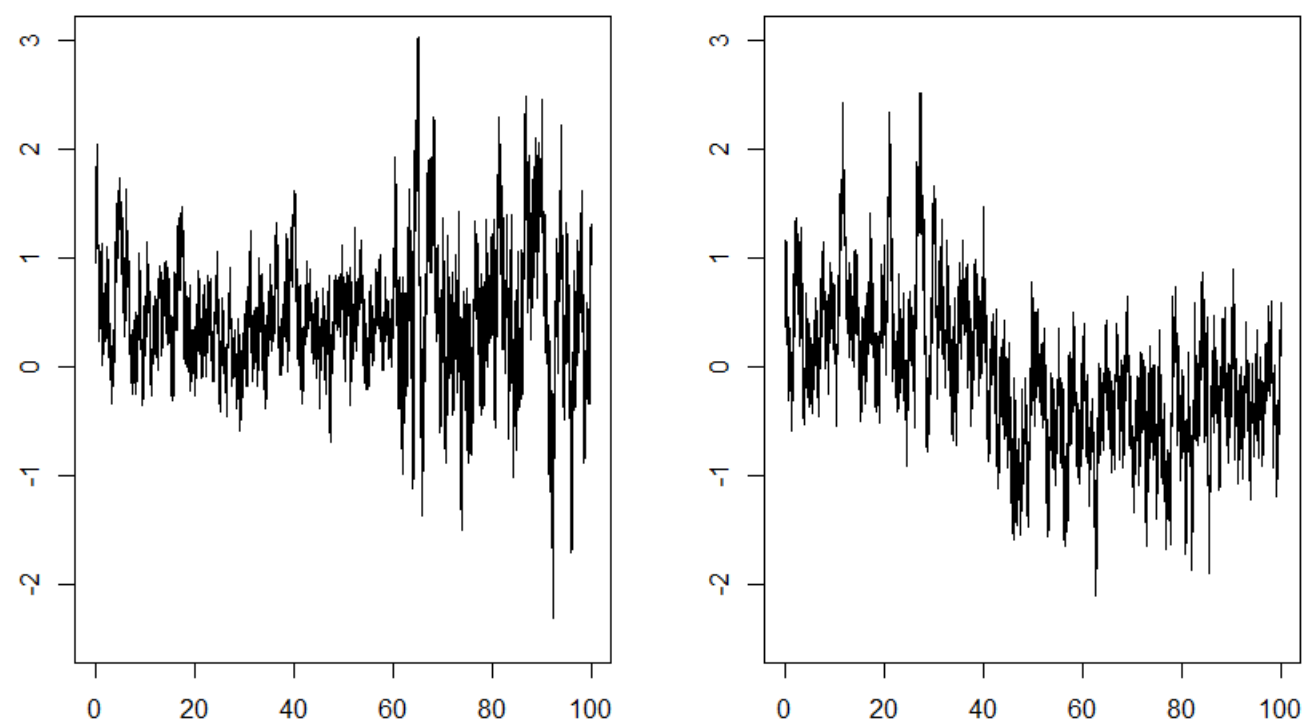

Figure 1. Sample paths of the hyperbolic diffusion model $\mathrm{d} X_{t}=\left(\beta+\frac{\gamma X_{t}}{\sqrt{1+X_{t}^{2}}}\right) \mathrm{d} t+\alpha \mathrm{d} W_{t}$ whose parameter changes form $(\alpha, \beta, \gamma)=(0.75,1,3)$ to $(1.5,1,3)$ at $t=60$ (left), and changes from $(\alpha, \beta, \gamma)=(1,1,3)$ to $(1,-1,3)$ at $t=40$ (right).
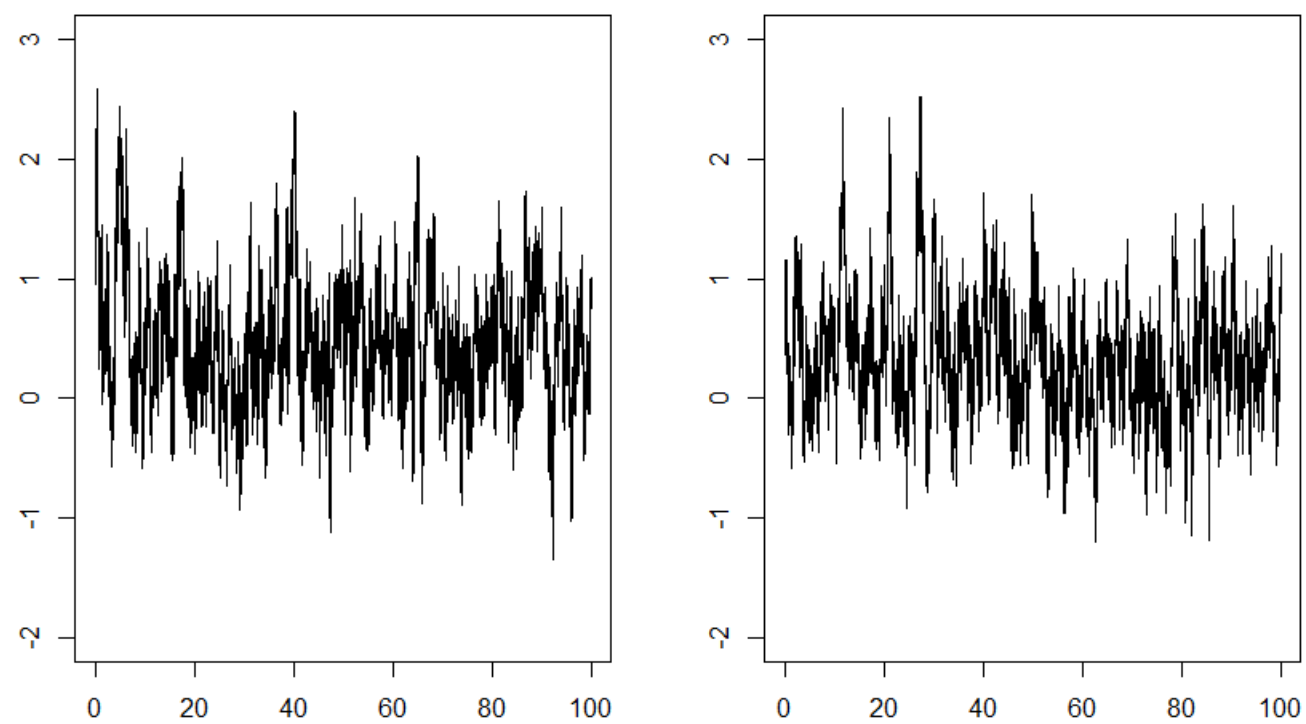

Figure 2. Sample paths of the hyperbolic diffusion model $\mathrm{d} X_{t}=\left(\beta+\frac{\gamma X_{t}}{\sqrt{1+X_{t}^{2}}}\right) \mathrm{d} t+\alpha \mathrm{d} W_{t}$ whose parameter changes form $(\alpha, \beta, \gamma)=(1,1,3)$ to $(1.05,1,3)$ at $t=30$ (left), and changes from $(\alpha, \beta, \gamma)=(1,1,3)$ to $(1,0.5,3)$ at $t=50$ (right). 
(2012) studied the estimating problem of the change point in diffusion parameter. In contrast, Song and Lee (2009), Lee (2011) and Song (2020) considered the detection of the parameter change in diffusion parameter, and Negri and Nishiyama (2017) and Tonaki et al. (2020) considered the detection of the parameter change in diffusion and drift parameters. Specifically, Negri and Nishiyama (2017) proposed simultaneous test for changes in diffusion and drift parameters, and Tonaki et al. (2020) proposed adaptive tests for those changes.

When we consider the change point problem of the diffusion processes, we first need to investigate whether there are any change points in the given data. Negri and Nishiyama (2017) considered the following hypothesis testing problem:

$$
H_{0}^{\theta}: \theta=(\alpha, \beta) \text { does not change over } 0 \leq t \leq T \quad \text { v.s. } \quad H_{1}^{\theta}: \text { not } H_{0}^{\theta} .
$$

They considered the simultaneous test for changes in diffusion and drift parameters, but even if any changes are detected, we can not determine which parameter changed. In order to solve this problem, Tonaki et al. (2020) considered the adaptive tests for changes in diffusion and drift parameters. Specifically, consider the following steps. First, consider the following hypothesis testing problem.

$$
H_{0}^{\alpha}: \alpha \text { does not change over } 0 \leq t \leq T \text { v.s. } H_{1}^{\alpha}: \text { not } H_{0}^{\alpha} .
$$

If $H_{0}^{\alpha}$ is not rejected, then consider the following hypothesis testing problem.

$$
H_{0}^{\beta}: \beta \text { does not change over } 0 \leq t \leq T \text { v.s. } H_{1}^{\beta}: \operatorname{not} H_{0}^{\beta} .
$$

When we find that there is a change, the next task is to estimate the change point. Since the study of the estimation of the change point for ergodic diffusion processes is still in its infancy, we consider the estimation of the change point for ergodic diffusion processes. With the adaptive detection method, when $H_{0}^{\alpha}$ is rejected, we can consider the estimation of the change point of the diffusion parameter $\alpha$, and when $H_{0}^{\alpha}$ is not rejected and $H_{0}^{\beta}$ is rejected, we can consider the estimation of the change point of the drift parameter $\beta$, which brings us one step closer to our goal.

This paper is organized as follows. In Section 2, we state the main results. We propose change point estimators for diffusion and drift parameters and present rates of convergence and distributional results of change point estimators. Section 3 discusses the nuisance parameters of the proposed estimators. Section 4 presents the powers of tests proposed by Tonaki et al. (2020). In Sections 5 and 6 , we give some examples and simulation studies. Finally, we provide the proofs in Section 7 .

\section{Main Results}

We consider the following two situations:

Situation I. $\alpha^{*}$ changes in $0 \leq t \leq T$, that is, there exists $\tau_{*}^{\alpha} \in(0,1)$ such that

$$
\alpha^{*}= \begin{cases}\alpha_{1}^{*}, & t \in\left[0, \tau_{*}^{\alpha} T\right), \\ \alpha_{2}^{*}, & t \in\left[\tau_{*}^{\alpha} T, T\right],\end{cases}
$$

where $\alpha_{1}^{*}, \alpha_{2}^{*} \in \operatorname{Int} \Theta_{A}, \alpha_{1}^{*} \neq \alpha_{2}^{*}$. Now 1.1 can be expressed as follows.

$$
X_{t}=\left\{\begin{array}{l}
X_{0}+\int_{0}^{t} b\left(X_{s}, \beta\right) \mathrm{d} s+\int_{0}^{t} a\left(X_{s}, \alpha_{1}^{*}\right) \mathrm{d} W_{s}, \quad t \in\left[0, \tau_{*}^{\alpha} T\right) \\
X_{\tau_{*}^{\alpha} T}+\int_{\tau_{*}^{\alpha} T}^{t} b\left(X_{s}, \beta\right) \mathrm{d} s+\int_{\tau_{*}^{\alpha} T}^{t} a\left(X_{s}, \alpha_{2}^{*}\right) \mathrm{d} W_{s} . \quad t \in\left[\tau_{*}^{\alpha} T, T\right]
\end{array}\right.
$$

Situation II. $\alpha^{*}$ does not change and $\beta^{*}$ changes in $0 \leq t \leq T$, that is, there exists $\tau_{*}^{\beta} \in(0,1)$ such that

$$
\beta^{*}= \begin{cases}\beta_{1}^{*}, & t \in\left[0, \tau_{*}^{\beta} T\right), \\ \beta_{2}^{*}, & t \in\left[\tau_{*}^{\beta} T, T\right],\end{cases}
$$

where $\beta_{1}^{*}, \beta_{2}^{*} \in \operatorname{Int} \Theta_{B}, \beta_{1}^{*} \neq \beta_{2}^{*}$. Now 1.1 can be expressed as follows.

$$
X_{t}=\left\{\begin{array}{l}
X_{0}+\int_{0}^{t} b\left(X_{s}, \beta_{1}^{*}\right) \mathrm{d} s+\int_{0}^{t} a\left(X_{s}, \alpha^{*}\right) \mathrm{d} W_{s}, \quad t \in\left[0, \tau_{*}^{\beta} T\right) \\
X_{\tau_{*}^{\beta} T}+\int_{\tau_{*}^{\beta} T}^{t} b\left(X_{s}, \beta_{2}^{*}\right) \mathrm{d} s+\int_{\tau_{*}^{\beta} T}^{t} a\left(X_{s}, \alpha^{*}\right) \mathrm{d} W_{s} . \quad t \in\left[\tau_{*}^{\beta} T, T\right]
\end{array}\right.
$$

We consider the following two cases:

Case A. The parameters $\alpha_{1}^{*}$ and $\alpha_{2}^{*}$ (resp. $\beta_{1}^{*}$ and $\beta_{2}^{*}$ ) depend on $n$ in Situation I (resp. II), 
Case B. The parameters $\alpha_{1}^{*}$ and $\alpha_{2}^{*}$ (resp. $\beta_{1}^{*}$ and $\beta_{2}^{*}$ ) are fixed and not depend on $n$ in Situation I (resp. II).

For matrices $c \in \mathbb{R}^{d_{1}} \otimes \mathbb{R}^{d_{2}}$, we write $c^{\otimes 2}=c c^{\top}$, where $c^{\top}$ is the transpose of $c$. Let $A(x, \alpha)=a(x, \alpha)^{\otimes 2}$ and $\Delta X_{i}=X_{t_{i}}-X_{t_{i-1}}$. Let $C_{\uparrow}^{k, \ell}\left(\mathbb{R}^{d} \times \Theta\right)$ be the space of all functions $f$ satisfying the following conditions:

(i) $f$ is continuously differentiable with respect to $x \in \mathbb{R}^{d}$ up to order $k$ for all $\theta \in \Theta$;

(ii) $f$ and all its $x$-derivatives up to order $k$ are $\ell$ times continuously differentiable with respect to $\theta \in \Theta$;

(iii) $f$ and all derivatives are of polynomial growth in $x \in \mathbb{R}^{d}$ uniformly in $\theta \in \Theta$, i.e., $g$ is of polynomial growth in $x \in \mathbb{R}^{d}$ uniformly in $\theta \in \Theta$ if, for some $C>0$, we have

$$
\sup _{\theta \in \Theta}\|g(x, \theta)\| \leq C(1+\|x\|)^{C} .
$$

We assume the following conditions:

[C1] There exists a constant $C$ such that for any $x, y \in \mathbb{R}^{d}$,

$$
\sup _{\alpha \in \Theta_{A}}\|a(x, \alpha)-a(y, \alpha)\|+\sup _{\beta \in \Theta_{B}}\|b(x, \beta)-b(y, \beta)\| \leq C\|x-y\| .
$$

[C2] $\sup _{t} \mathbb{E}_{\theta}\left[\left\|X_{t}\right\|^{k}\right]<\infty$ for all $k \geq 0$ and $\theta \in \Theta$.

[C3] $\inf _{x, \alpha} \operatorname{det}(A(x, \alpha))>0$.

[C4] $a \in C_{\uparrow}^{4,4}\left(\mathbb{R}^{d} \times \Theta_{A}\right)$ and $b \in C_{\uparrow}^{4,4}\left(\mathbb{R}^{d} \times \Theta_{B}\right)$.

[C5] The solution of (1.1) is ergodic with its invariant measure $\mu_{\theta}$ such that

$$
\int_{\mathbb{R}^{d}}\|x\|^{k} \mathrm{~d} \mu_{\theta}(x)<\infty \text { for all } k \geq 0 \text { and } \theta \in \Theta
$$

and

$$
\int_{\mathbb{R}^{d}} f(x) \mathrm{d} \mu_{\theta_{n}}(x) \longrightarrow \int_{\mathbb{R}^{d}} f(x) \mathrm{d} \mu_{\theta_{0}}(x) \quad \text { for all measurable function } f
$$

as $\theta_{n} \longrightarrow \theta_{0}$.

2.1. Estimation for the diffusion parameter. First, we consider Situation I, that is, the estimation for the diffusion parameter. Write $\vartheta_{\alpha}=\left|\alpha_{1}^{*}-\alpha_{2}^{*}\right|$ and let

$$
\begin{aligned}
\Xi^{\alpha}(x, \alpha) & =\left[\operatorname{tr}\left(A^{-1} \partial_{\alpha^{\ell_{1}}} A A^{-1} \partial_{\alpha^{\ell_{2}}} A(x, \alpha)\right)\right]_{\ell_{1}, \ell_{2}=1}^{p}, \\
\Gamma^{\alpha}\left(x, \alpha_{1}, \alpha_{2}\right) & =\operatorname{tr}\left(A^{-1}\left(x, \alpha_{1}\right) A\left(x, \alpha_{2}\right)-I_{d}\right)-\log \operatorname{det} A^{-1}\left(x, \alpha_{1}\right) A\left(x, \alpha_{2}\right) .
\end{aligned}
$$

Now we additionally assume the following conditions:

[C6-I] There exist estimators $\hat{\alpha}_{k}=\hat{\alpha}_{k, n}(k=1,2)$ such that

$$
\sqrt{n}\left(\hat{\alpha}_{k}-\alpha_{k}^{*}\right)=O_{p}(1) .
$$

[A1-I] $\alpha_{1}^{*}$ and $\alpha_{2}^{*}$ depend on $n$, and $\vartheta_{\alpha}=\vartheta_{\alpha, n}$ satisfies the following.

$$
\vartheta_{\alpha} \longrightarrow 0, \quad n \vartheta_{\alpha}^{2} \longrightarrow \infty, \quad \frac{h}{\vartheta_{\alpha}^{2}} \longrightarrow \infty, \quad T \vartheta_{\alpha} \longrightarrow 0
$$

as $n \rightarrow \infty$, and $\vartheta_{\alpha}^{-1}\left(\alpha_{k}^{*}-\alpha_{0}\right)=O(1)$, where $\alpha_{0} \in \operatorname{Int} \Theta_{A}$,

[A2-I] For the following three functions and for any $r \in(1,2)$ such that $n h^{r} \longrightarrow \infty$,

$$
\max _{\left[n^{1 / r}\right] \leq k \leq n}\left|\frac{1}{k} \sum_{i=\left[n \tau_{*}^{\alpha}\right]+1}^{\left[n \tau_{*}^{\alpha}\right]+k} f\left(X_{t_{i-1}}\right)-\int_{\mathbb{R}^{d}} f(x) \mathrm{d} \mu_{\alpha_{0}}(x)\right| \stackrel{p}{\longrightarrow} 0 .
$$

(a) $\Xi^{\alpha}\left(x, \alpha_{0}\right)$,

(b) $\partial_{\alpha} \Xi^{\alpha}\left(x, \alpha_{0}\right)$,

(c) $\left.\partial_{\alpha}^{3}\left(\operatorname{tr}\left(A^{-1}(x, \alpha) A\left(x, \alpha_{0}\right)\right)-\log \operatorname{det} A^{-1}(x, \alpha) A\left(x, \alpha_{0}\right)\right)\right|_{\alpha=\alpha_{0}}$.

$[\mathbf{B} 1-\mathbf{I}] \inf _{x} \Gamma^{\alpha}\left(x, \alpha_{1}^{*}, \alpha_{2}^{*}\right)>0$.

[B2-I] $\stackrel{x}{\text { There exists a constant } C>0 \text { such that }}$
(a) $\sup _{x, \alpha_{k}}\left|\partial_{\left(\alpha_{1}, \alpha_{2}\right)} \Gamma^{\alpha}\left(x, \alpha_{1}, \alpha_{2}\right)\right|<C$, 
(b) $\sup _{x, \alpha_{k}}\left|\left[\operatorname{tr}\left(\left(A^{-1}\left(x, \alpha_{1}\right)-A^{-1}\left(x, \alpha_{2}\right)\right) \partial_{\alpha^{\ell}} A\left(x, \alpha_{3}\right)\right)\right]_{\ell=1}^{p}\right|<C$

(c) $\sup _{x, \theta}|Q(x, \theta)|<C$,

where $Q(x, \theta)$ is the coefficient of $h^{2}$ of $\mathbb{E}_{\theta}\left[\left(X_{t_{i}}-X_{t_{i-1}}\right)^{\otimes 2} \mid \mathscr{G}_{i-1}^{n}\right]$, that is, $\mathbb{E}_{\theta}\left[\left(X_{t_{i}}-X_{t_{i-1}}\right)^{\otimes 2} \mid \mathscr{G}_{i-1}^{n}\right]=$ $h A\left(X_{t_{i-1}}, \alpha\right)+h^{2} Q\left(X_{t_{i-1}}, \theta\right)+\cdots$. Here, $\mathscr{G}_{i-1}^{n}=\sigma\left[\left\{W_{s}\right\}_{s \leq t_{i}^{n}}\right]$.

Remark 1 See Section 3 for how to construct the estimators $\hat{\alpha}_{k}$ that satisfy [C6-I]. If $a(x, \alpha)=\sigma(x) c(\alpha)$ for $\sigma: \mathbb{R}^{d} \longrightarrow \mathbb{R}^{d} \otimes \mathbb{R}^{d}, c: \mathbb{R}^{p} \longrightarrow \mathbb{R}^{d} \otimes \mathbb{R}^{d}$, then [A2-I], [B1-I] and [B2-I](a),(b) hold because the functions of (a)-(c) of [A2-I], $\Gamma^{\alpha}\left(x, \alpha_{1}, \alpha_{2}\right), \partial_{\left(\alpha_{1}, \alpha_{2}\right)} \Gamma^{\alpha}\left(x, \alpha_{1}, \alpha_{2}\right)$ and

$$
\left[\operatorname{tr}\left(\left(A^{-1}\left(x, \alpha_{1}\right)-A^{-1}\left(x, \alpha_{2}\right)\right) \partial_{\alpha^{\ell}} A\left(x, \alpha_{3}\right)\right)\right]_{\ell=1}^{p}
$$

do not depend on $x$. Therefore, the Ornstein-Uhlenbeck process and hyperbolic diffusion model, which will be described later in Section 5, are models that satisfy [A2-I], and these models are examples in Case A. Hyperbolic diffusion model also satisfies [B2-I](c), and this model is an example in Case B, see Section 5.5. If the diffusion coefficient is $a(x, \alpha)=\alpha$, then $[\mathbf{A 1 - I}]$ and $[\mathbf{A 2 - I}]$ can be reduced to the following condition: [A1'-I] $\alpha_{1}^{*}$ and $\alpha_{2}^{*}$ depend on $n$, and $\vartheta_{\alpha}=\vartheta_{\alpha, n}$ satisfies the following.

$$
\vartheta_{\alpha} \longrightarrow 0, \quad n \vartheta_{\alpha}^{2} \longrightarrow \infty, \quad T \vartheta_{\alpha} \longrightarrow 0
$$

as $n \rightarrow \infty$, and $\vartheta_{\alpha}^{-1}\left(\alpha_{k}^{*}-\alpha_{0}\right)=O(1)$, where $\alpha_{0} \in \operatorname{Int} \Theta_{A}$.

Therefore, if $\left\{X_{t}\right\}_{t \geq 0}$ after the parameter change is stationary, then [A1-I] and [A2-I] can be reduced to the following condition:

[A1"-I] $\alpha_{1}^{*}$ and $\alpha_{2}^{*}$ depend on $n$, and $\vartheta_{\alpha}=\vartheta_{\alpha, n}$ satisfies the following.

$$
\vartheta_{\alpha} \longrightarrow 0, \quad n \vartheta_{\alpha}^{2} \longrightarrow \infty, \quad \frac{h}{\vartheta_{\alpha}^{2}} \longrightarrow \infty
$$

as $n \rightarrow \infty$, and $\vartheta_{\alpha}^{-1}\left(\alpha_{k}^{*}-\alpha_{0}\right)=O(1)$, where $\alpha_{0} \in \operatorname{Int} \Theta_{A}$,

That is, if $\left\{X_{t}\right\}_{t \geq 0}$ after the parameter change is stationary and we assume [A1'-I], then [A1-I] and [A2-I] hold, see Section 5.1 .

In Situation I, we set

$$
\begin{aligned}
& F_{i}(\alpha)=\operatorname{tr}\left(A^{-1}\left(X_{t_{i-1}}, \alpha\right) \frac{\left(\Delta X_{i}\right)^{\otimes 2}}{h}\right)+\log \operatorname{det} A\left(X_{t_{i-1}}, \alpha\right), \\
& \Phi_{n}\left(\tau: \alpha_{1}, \alpha_{2}\right)=\sum_{i=1}^{[n \tau]} F_{i}\left(\alpha_{1}\right)+\sum_{i=[n \tau]+1}^{n} F_{i}\left(\alpha_{2}\right)
\end{aligned}
$$

and propose

$$
\hat{\tau}_{n}^{\alpha}=\underset{\tau \in[0,1]}{\operatorname{argmin}} \Phi_{n}\left(\tau: \hat{\alpha}_{1}, \hat{\alpha}_{2}\right)
$$

as an estimator of $\tau_{*}^{\alpha}$.

In Case $\mathrm{A}$, we set for $v \in \mathbb{R}$,

$$
\begin{aligned}
e_{\alpha} & =\lim _{n \rightarrow \infty} \vartheta_{\alpha}^{-1}\left(\alpha_{1}^{*}-\alpha_{2}^{*}\right), \\
\mathcal{J}_{\alpha} & =\frac{1}{2} e_{\alpha}^{\top} \int_{\mathbb{R}^{d}} \Xi^{\alpha}\left(x, \alpha_{0}\right) \mathrm{d} \mu_{\alpha_{0}}(x) e_{\alpha}, \\
\mathbb{F}(v) & =-2 \mathcal{J}_{\alpha}^{1 / 2} \mathcal{W}(v)+\mathcal{J}_{\alpha}|v|,
\end{aligned}
$$

where $\mathcal{W}$ is a two sided standard Wiener process.

Theorem 1 Suppose that $[\mathbf{C 1}]-[\mathbf{C 5}]$ and [C6-I] hold in Situation I. Then, under [A1-I] and [A2-I] in Case A,

$$
n \vartheta_{\alpha}^{2}\left(\hat{\tau}_{n}^{\alpha}-\tau_{*}^{\alpha}\right) \stackrel{d}{\longrightarrow} \underset{v \in \mathbb{R}}{\operatorname{argmin}} \mathbb{F}(v)
$$


Theorem 2 Suppose that [C1]-[C5] and [C6-I] hold in Situation I. Then, under [B1-I] and [B2-I] in Case B,

$$
n\left(\hat{\tau}_{n}^{\alpha}-\tau_{*}^{\alpha}\right)=O_{p}(1)
$$

Corollary 1 Suppose that [C1]-[C5] and [C6-I] hold in Situation I. If for $\epsilon_{1} \in[0,1)$ there exists $\delta_{1} \in$ $\left(0, \frac{1-\epsilon_{1}}{2}\right)$ such that $n h^{1 /\left(\epsilon_{1}+\delta_{1}\right)} \longrightarrow 0$, then, under [B1-I], [B2-I] (a) and (b) in Case B,

$$
n^{\epsilon_{1}}\left(\hat{\tau}_{n}^{\alpha}-\tau_{*}^{\alpha}\right)=o_{p}(1) .
$$

Particularly, for $\epsilon_{1} \in\left[0, \frac{1}{2}\right)$ there exists always $\delta_{1}$ regardless of $h$.

Remark 2 Although we could not specify the distribution of $n\left(\hat{\tau}_{n}^{\alpha}-\tau_{*}^{\alpha}\right)$ in Case B, we have strong results in this case. Actually, in Case B, we see $\hat{\tau}_{n}^{\alpha}-\tau_{*}^{\alpha}=O_{p}\left(n^{-1}\right)$. However, if we choose $\vartheta_{\alpha}=n^{-\nu}, 0<\nu<\frac{1}{2}$ that satisfies [A1-I], we have $n^{1-2 \nu}\left(\hat{\tau}_{n}^{\alpha}-\tau_{*}^{\alpha}\right)=O_{p}(1)$ in Case A, but $n^{1-2 \nu}\left(\hat{\tau}_{n}^{\alpha}-\tau_{*}^{\alpha}\right)=o_{p}(1)$ in Case B.

2.2. Estimation for the drift parameter. Next, we consider Situation II, that is, the estimation for the drift parameter. Write $\vartheta_{\beta}=\left|\beta_{1}^{*}-\beta_{2}^{*}\right|$ and let

$$
\begin{aligned}
\Xi^{\beta}(x, \alpha, \beta) & =\left[\partial_{\beta^{\ell_{1}}} b(x, \beta)^{\top} A^{-1}(x, \alpha) \partial_{\beta^{\ell_{2}}} b(x, \beta)\right]_{\ell_{1}, \ell_{2}=1}^{q}, \\
\Gamma^{\beta}\left(x, \alpha, \beta_{1}, \beta_{2}\right) & =\operatorname{tr}\left[A^{-1}(x, \alpha)\left(b\left(x, \beta_{1}\right)-b\left(x, \beta_{2}\right)\right)^{\otimes 2}\right] .
\end{aligned}
$$

Now we additionally assume the following conditions:

[C6-II] There exist estimators $\hat{\alpha}=\hat{\alpha}_{n}, \hat{\beta}_{k}=\hat{\beta}_{k, n}(k=1,2)$ such that

$$
\sqrt{n}\left(\hat{\alpha}-\alpha^{*}\right)=O_{p}(1), \quad \sqrt{T}\left(\hat{\beta}_{k}-\beta_{k}^{*}\right)=O_{p}(1) .
$$

[A1-II] $\beta_{1}^{*}$ and $\beta_{2}^{*}$ depend on $n$ and $\vartheta_{\beta}=\vartheta_{\beta, n}$ satisfies the following.

$$
\vartheta_{\beta} \longrightarrow 0, \quad T \vartheta_{\beta}^{2} \longrightarrow \infty, \quad T \vartheta_{\beta}^{4} \longrightarrow 0
$$

as $n \rightarrow \infty$, and $\vartheta_{\beta}^{-1}\left(\beta_{k}^{*}-\beta_{0}\right)=O(1)$, where $\beta_{0} \in \operatorname{Int} \Theta_{B}$.

[A2-II] For the following three functions and for any $r \in(1,2)$ such that $n h^{r} \longrightarrow \infty$,

$$
\max _{\left[n^{1 / r}\right] \leq k \leq n}\left|\frac{1}{k} \sum_{i=\left[n \tau_{*}^{\beta}\right]+1}^{\left[n \tau_{*}^{\beta}\right]+k} f\left(X_{t_{i-1}}\right)-\int_{\mathbb{R}^{d}} f(x) \mathrm{d} \mu_{\left(\alpha^{*}, \beta_{0}\right)}(x)\right| \stackrel{p}{\longrightarrow} 0 .
$$
(a) $\Xi^{\beta}\left(x, \alpha^{*}, \beta_{0}\right)$,
(b) $\partial_{\beta} \Xi^{\beta}\left(x, \alpha^{*}, \beta_{0}\right)$,
(c) $\partial_{\beta}^{3}\left(\left.\operatorname{tr}\left(A^{-1}\left(x, \alpha^{*}\right)\left(b(x, \beta)-b\left(x, \beta_{0}\right)\right)^{\otimes 2}\right)\right|_{\beta=\beta_{0}}\right.$.

[A3-II] There exists an integer $m \geq 4$ such that $n^{m / 2-1} h^{(m-1) / 2} \longrightarrow \infty, h^{-1 / 2} \vartheta_{\beta}^{m-2} \longrightarrow 0$ and $b \in C_{\uparrow}^{4, m}\left(\mathbb{R}^{d} \times\right.$ $\left.\Theta_{B}\right)$.

[B1-II] $\inf _{x} \Gamma^{\beta}\left(x, \alpha^{*}, \beta_{1}^{*}, \beta_{2}^{*}\right)>0$.

[B2-II] There exists a constant $C>0$ such that
(a) $\sup _{x, \alpha, \beta_{k}}\left|\partial_{\left(\alpha, \beta_{1}, \beta_{2}\right)} \Gamma^{\beta}\left(x, \alpha, \beta_{1}, \beta_{2}\right)\right|<C$,
(b) $\sup _{x, \alpha, \beta_{k}}\left|\left[\operatorname{tr}\left(A^{-1}(x, \alpha) \partial_{\beta^{\ell}} b\left(x, \beta_{1}\right)\left(b\left(x, \beta_{2}\right)-b\left(x, \beta_{3}\right)\right)^{\top}\right)\right]_{\ell=1}^{q}\right|<C$.

Remark 3 See Section 3 for how to construct the estimators $\hat{\beta}_{k}$ that satisfy [C6-II]. If $\left\{X_{t}\right\}_{t \geq 0}$ after the parameter change is stationary, then $[\mathbf{A} 1-\mathbf{I I}]$ and $[\mathbf{A 2}-\mathbf{I I}]$ can be reduced to the following condition:

[A1'-II $] \beta_{1}^{*}$ and $\beta_{2}^{*}$ depend on $n$ and $\vartheta_{\beta}=\vartheta_{\beta, n}$ satisfies the following.

$$
\vartheta_{\beta} \longrightarrow 0, \quad T \vartheta_{\beta}^{2} \longrightarrow \infty
$$

as $n \rightarrow \infty$, and $\vartheta_{\beta}^{-1}\left(\beta_{k}^{*}-\beta_{0}\right)=O(1)$, where $\beta_{0} \in \operatorname{Int} \Theta_{B}$.

That is, if $\left\{X_{t}\right\}_{t \geq 0}$ after the parameter change is stationary and we assume [A1'-II], then [A1-II] and [A2-II] hold, see Section 5.1. The hyperbolic diffusion model is one of the models that satisfy [B1-II] and [B2-II], see Section 5.5 . 
In Situation II, we set

$$
\begin{aligned}
& G_{i}(\beta \mid \alpha)=\operatorname{tr}\left(A^{-1}\left(X_{t_{i-1}}, \alpha\right) \frac{\left(\Delta X_{i}-h b\left(X_{t_{i-1}}, \beta\right)\right)^{\otimes 2}}{h}\right) \\
& \Psi_{n}\left(\tau: \beta_{1}, \beta_{2} \mid \alpha\right)=\sum_{i=1}^{[n \tau]} G_{i}\left(\beta_{1} \mid \alpha\right)+\sum_{i=[n \tau]+1}^{n} G_{i}\left(\beta_{2} \mid \alpha\right)
\end{aligned}
$$

and propose

$$
\hat{\tau}_{n}^{\beta}=\underset{\tau \in[0,1]}{\operatorname{argmin}} \Psi_{n}\left(\tau: \hat{\beta}_{1}, \hat{\beta}_{2} \mid \hat{\alpha}\right)
$$

as an estimator of $\tau_{*}^{\beta}$.

In Case $\mathrm{A}$, we set for $v \in \mathbb{R}$,

$$
\begin{aligned}
e_{\beta} & =\lim _{n \rightarrow \infty} \vartheta_{\beta}^{-1}\left(\beta_{1}^{*}-\beta_{2}^{*}\right), \\
\mathcal{J}_{\beta} & =e_{\beta}^{\top} \int_{\mathbb{R}^{d}} \Xi^{\beta}\left(x, \alpha^{*}, \beta_{0}\right) \mathrm{d} \mu_{\left(\alpha^{*}, \beta_{0}\right)}(x) e_{\beta}, \\
\mathbb{G}(v) & =-2 \mathcal{J}_{\beta}^{1 / 2} \mathcal{W}(v)+\mathcal{J}_{\beta}|v| .
\end{aligned}
$$

Theorem 3 Suppose that [C1]-[C5] and [C6-II] hold in Situation II. Then, under [A1-II]-[A3-II] in Case A,

$$
T \vartheta_{\beta}^{2}\left(\hat{\tau}_{n}^{\beta}-\tau_{*}^{\beta}\right) \stackrel{d}{\longrightarrow} \underset{v \in \mathbb{R}}{\operatorname{argmin}} \mathbb{G}(v) .
$$

Theorem 4 Suppose that [C1]-[C5] and [C6-II] hold in Situation II. Then, under [B1-II], [B2-II] in Case B,

$$
T\left(\hat{\tau}_{n}^{\beta}-\tau_{*}^{\beta}\right)=O_{p}(1)
$$

Remark 4 Although we could not specify the distribution of $T\left(\hat{\tau}_{n}^{\beta}-\tau_{*}^{\beta}\right)$ in Case B, we have strong results in this case. Actually, in Case B, we see $\hat{\tau}_{n}^{\beta}-\tau_{*}^{\beta}=O_{p}\left(T^{-1}\right)$. However, if we choose $\vartheta_{\beta}=T^{-\nu}, 0<\nu<\frac{1}{2}$ that satisfies $[\mathbf{A 1 - I I}]$, we have $T^{1-2 \nu}\left(\hat{\tau}_{n}^{\beta}-\tau_{*}^{\beta}\right)=O_{p}(1)$ in Case A, but $T^{1-2 \nu}\left(\hat{\tau}_{n}^{\beta}-\tau_{*}^{\beta}\right)=o_{p}(1)$ in Case B.

\section{Estimation of the nuisance parameters $\alpha_{k}^{*}, \beta_{k}^{*}$}

When the values of the parameters $\alpha_{k}^{*}, \beta_{k}^{*}$ are unknown, it is necessary to estimate the parameters $\alpha_{k}^{*}, \beta_{k}^{*}$ for the change point estimation. In this section, we will discuss estimation of nuisance parameters $\alpha_{k}^{*}, \beta_{k}^{*}$.

First, we need the following information to estimate $\alpha_{k}^{*}$ or $\beta_{k}^{*}$ :

[D1] There exist $\underline{\tau}^{\alpha}, \bar{\tau}^{\alpha} \in(0,1)$ such that $\tau_{*}^{\alpha} \in\left[\underline{\tau}^{\alpha}, \bar{\tau}^{\alpha}\right]$.

[D2] There exist $\underline{\tau}^{\beta}, \bar{\tau}^{\beta} \in(0,1)$ such that $\tau_{*}^{\beta} \in\left[\underline{\tau}^{\beta}, \bar{\tau}^{\beta}\right]$.

If this information is obtained, since there are no change points in the intervals $\left[0, \tau^{\alpha} T\right]$ and $\left[\bar{\tau}^{\alpha} T, T\right]$, we can estimate $\alpha_{1}^{*}$ from the data of $\left[0, \tau^{\alpha} T\right]$ and $\alpha_{2}^{*}$ from the data of $\left[\bar{\tau}^{\alpha} T, T\right]$. For example, we can construct estimators that satisfy $[\mathbf{C 6 - I}]$ or $[\mathbf{C 6 - I I}]$ by the following procedures. In Situation I, set

$$
\begin{aligned}
& \underline{U}_{n}^{(1)}(\alpha)=\sum_{i=1}^{\left[n \underline{\tau}^{\alpha}\right]}\left(\operatorname{tr}\left(A^{-1}\left(X_{t_{i-1}}, \alpha\right) \frac{\left(\Delta X_{i}\right)^{\otimes 2}}{h}\right)+\log \operatorname{det} A\left(X_{t_{i-1}}, \alpha\right)\right) \\
& \bar{U}_{n}^{(1)}(\alpha)=\sum_{i=\left[n \bar{\tau}^{\alpha}\right]+1}^{n}\left(\operatorname{tr}\left(A^{-1}\left(X_{t_{i-1}}, \alpha\right) \frac{\left(\Delta X_{i}\right)^{\otimes 2}}{h}\right)+\log \operatorname{det} A\left(X_{t_{i-1}}, \alpha\right)\right),
\end{aligned}
$$

and let $\hat{\alpha}_{1}=\operatorname{arginf}_{\alpha} \underline{U}_{n}^{(1)}(\alpha)$, and $\hat{\alpha}_{2}=\operatorname{arginf}_{\alpha} \bar{U}_{n}^{(1)}(\alpha)$ as estimators of $\alpha_{1}^{*}$ and $\alpha_{2}^{*}$, respectively. Then, we have

$$
\sqrt{n}\left(\hat{\alpha}_{1}-\alpha_{1}^{*}\right)=O_{p}(1), \quad \sqrt{n}\left(\hat{\alpha}_{2}-\alpha_{2}^{*}\right)=O_{p}(1)
$$


Similarly in Situation II, set

$$
\begin{aligned}
U_{n}^{(1)}(\alpha) & =\sum_{i=1}^{n}\left(\operatorname{tr}\left(A_{i-1}^{-1}(\alpha) \frac{\left(\Delta X_{i}\right)^{\otimes 2}}{h}\right)+\log \operatorname{det} A_{i-1}(\alpha)\right), \\
\underline{U}_{n}^{(2)}(\beta \mid \alpha) & =\sum_{i=1}^{\left[n \underline{\tau}^{\beta}\right]} \operatorname{tr}\left(A_{i-1}^{-1}(\alpha) \frac{\left(\Delta X_{i}-h b_{i-1}(\beta)\right)^{\otimes 2}}{h}\right), \\
\bar{U}_{n}^{(2)}(\beta \mid \alpha) & =\sum_{i=\left[n \bar{\tau}^{\beta}\right]+1}^{n} \operatorname{tr}\left(A_{i-1}^{-1}(\alpha) \frac{\left(\Delta X_{i}-h b_{i-1}(\beta)\right)^{\otimes 2}}{h}\right),
\end{aligned}
$$

and let $\hat{\alpha}=\operatorname{arginf}_{\alpha} U_{n}^{(1)}(\alpha), \hat{\beta}_{1}=\operatorname{arginf}_{\beta} \underline{U}_{n}^{(2)}(\beta \mid \hat{\alpha})$, and $\hat{\beta}_{2}=\operatorname{arginf}_{\beta} \bar{U}_{n}^{(2)}(\beta \mid \hat{\alpha})$ as estimators of $\alpha^{*}, \beta_{1}^{*}$ and $\beta_{2}^{*}$, respectively. Then, we have

$$
\sqrt{T}\left(\hat{\beta}_{1}-\beta_{1}^{*}\right)=O_{p}(1), \quad \sqrt{T}\left(\hat{\beta}_{2}-\beta_{2}^{*}\right)=O_{p}(1) .
$$

Thus, if [D1] or [D2] is satisfied, then we can construct estimators that satisfy [C6-I] or [C6-II]. Next, let us discuss how to find $\underline{\tau}$ and $\bar{\tau}$ that satisfy [D1] or [D2]. To find these, we need some method to detect changes in the diffusion or drift parameters. However, in Situations I and II, the adaptive tests for changes in diffusion and drift parameters proposed by Tonaki et al. (2020) makes it possible. Namely, we can detect a change in the diffusion or drift parameters in the interval $\left[\tau_{1} T, \tau_{2} T\right]$ by the following test statistics $\mathcal{T}_{n}^{\alpha}\left(\tau_{1}, \tau_{2}\right)$, $\mathcal{T}_{1, n}^{\beta}\left(\tau_{1}, \tau_{2}\right)$ and $\mathcal{T}_{2, n}^{\beta}\left(\tau_{1}, \tau_{2}\right)$.

$$
\begin{aligned}
\mathcal{T}_{n}^{\alpha}\left(\tau_{1}, \tau_{2}\right) & =\frac{1}{\sqrt{2 d\left(\left[n \tau_{2}\right]-\left[n \tau_{1}\right]\right)}} \max _{1 \leq k \leq\left[n \tau_{2}\right]-\left[n \tau_{1}\right]}\left|\sum_{i=\left[n \tau_{1}\right]+1}^{\left[n \tau_{1}\right]+k} \hat{\eta}_{i}-\frac{k}{\left[n \tau_{2}\right]-\left[n \tau_{1}\right]} \sum_{i=\left[n \tau_{1}\right]+1}^{\left[n \tau_{2}\right]} \hat{\eta}_{i}\right|, \\
\hat{\eta}_{i} & =\operatorname{tr}\left(A^{-1}\left(X_{t_{i-1}}, \hat{\alpha}\right) \frac{\left(\Delta X_{i}\right)^{\otimes 2}}{h}\right), \\
\mathcal{T}_{1, n}^{\beta}\left(\tau_{1}, \tau_{2}\right) & =\frac{1}{\sqrt{d T\left(\tau_{2}-\tau_{1}\right)}} \max _{1 \leq k \leq\left[n \tau_{2}\right]-\left[n \tau_{1}\right]}\left|\sum_{i=\left[n \tau_{1}\right]+1}^{\left[n \tau_{1}\right]+k} \hat{\xi}_{i}-\frac{k}{\left[n \tau_{2}\right]-\left[n \tau_{1}\right]} \sum_{i=\left[n \tau_{1}\right]+1}^{\left[n \tau_{2}\right]} \hat{\xi}_{i}\right|, \\
\hat{\xi}_{i} & =1_{d}^{\top} a^{-1}\left(X_{t_{i-1}}, \hat{\alpha}\right)\left(X_{t_{i}}-X_{t_{i-1}}-h b\left(X_{t_{i-1}}, \hat{\beta}\right)\right), \\
\mathcal{T}_{2, n}^{\beta}\left(\tau_{1}, \tau_{2}\right) & =\frac{1}{\sqrt{T\left(\tau_{2}-\tau_{1}\right)}} \max _{1 \leq k \leq\left[n \tau_{2}\right]-\left[n \tau_{1}\right]} \mid \mathcal{I}_{n}^{-1 / 2}\left(\tau_{1}, \tau_{2}\right)\left(\sum_{i=\left[n \tau_{1}\right]+1}^{\left[n \tau_{1}\right]+k} \hat{\zeta}_{i}-\frac{k}{\left[n \tau_{2}\right]-\left[n \tau_{1}\right]} \sum_{i=\left[n \tau_{1}\right]+1}^{\left[n \tau_{2}\right]} \hat{\zeta}_{i}\right) \|, \\
\hat{\zeta}_{i} & =\partial_{\beta} b\left(X_{t_{i-1},}, \hat{\beta}\right)^{\top} A^{-1}\left(X_{t_{i-1}}, \hat{\alpha}\right)\left(X_{t_{i}}-X_{t_{i-1}}-h b\left(X_{t_{i-1}}, \hat{\beta}\right)\right), \\
\mathcal{I}_{n}\left(\tau_{1}, \tau_{2}\right) & =\frac{1}{\left[n \tau_{2}\right]-\left[n \tau_{1}\right]} \sum_{i=\left[n \tau_{1}\right]+1}^{\left[n \tau_{2}\right]} \partial_{\beta} b\left(X_{t_{i-1}}, \hat{\beta}\right)^{\top} A^{-1}\left(X_{t_{i-1}}, \hat{\alpha}\right) \partial_{\beta} b\left(X_{t_{i-1}}, \hat{\beta}\right) .
\end{aligned}
$$

Below we describe how to find $\underline{\tau}$ and $\bar{\tau}$.

First, the assumption is that a change is detected in the interval $[0, T]$.

U-1) Choose $\tau_{1}^{U} \in(0,1)$ (e.g., $\tau_{1}^{U}=3 / 4$ ), and investigate the change point in the interval $\left[0, \tau_{1}^{U} T\right]$.

(a) If a change is detected, set $\bar{\tau}=\tau_{1}^{U}$ and go to L-1).

(b) If not detected, go to U-2).

U- $k$ ) Choose $\tau_{k}^{U} \in\left(\tau_{k-1}^{U}, 1\right)$ (e.g., $\left.\tau_{k}^{U}=1-2^{-(k+1)}\right)$, and investigate the change point in the interval $\left[0, \tau_{k}^{U} T\right]$.

(a) If a change is detected, set $\bar{\tau}=\tau_{k}^{U}$ and go to L-1).

(b) If not detected, go to $\mathrm{U}-(k+1))$.

Assume that we chose $\tau_{k}^{U}$ as $\bar{\tau}$ in U-k).

L-1) Choose $\tau_{1}^{L} \in\left(0, \tau_{k}^{U}\right.$ ) (e.g., $\tau_{1}^{L}=1 / 4$ or $\tau_{k-1}^{U}$ ), and investigate the change point in the interval $\left[\tau_{1}^{L} T, T\right]$.

(a) If a change is detected, set $\underline{\tau}=\tau_{1}^{L}$.

(b) If not detected, go to L-2). 
L- $\boldsymbol{m}$ ) Choose $\tau_{m}^{L} \in\left(0, \tau_{m-1}^{L}\right)$ (e.g., $\tau_{m}^{L}=2^{-(m+1)}$ or $\tau_{k-m}^{L}$ (if $\left.k>m\right)$ ), and investigate the change point in the interval $\left[\tau_{m}^{L} T, T\right]$.

(a) If a change is detected, set $\underline{\tau}=\tau_{m}^{L}$.

(b) If not detected, go to L- $(m+1))$.

We may also choose $\underline{\tau}$ and $\bar{\tau}$ at the same time, that is,

1) Choose $\tau_{1} \in(0,1 / 2)$ (e.g., $\left.\tau_{1}=1 / 4\right)$, and investigate the change point in the interval $\left[\tau_{1} T,\left(1-\tau_{1}\right) T\right]$.

(a) If a change is detected, set $\underline{\tau}=\tau_{1}$ and $\bar{\tau}=1-\tau_{1}$.

(b) If not detected, go to 2$)$.

k) Choose $\tau_{k} \in\left(0, \tau_{k-1}\right)$ (e.g., $\left.\tau_{k}=2^{-(k+1)}\right)$, and investigate the change point in the interval $\left[\tau_{k} T,(1-\right.$ $\left.\left.\tau_{k}\right) T\right]$.

(a) If a change is detected, set $\underline{\tau}=\tau_{k}$ and $\bar{\tau}=1-\tau_{k}$.

(b) If not detected, go to $k+1$ ).

We can choose $\underline{\tau}$ and $\bar{\tau}$ in the above manner.

\section{The powers of tests in CAse A}

As we saw in Section 3 we need estimators of the nuisance parameters $\alpha_{k}^{*}$ and $\beta_{k}^{*}$ when estimating the change point, and have to find $\underline{\tau}$ and $\bar{\tau}$ that satisfy [D1] or [D2] in order to construct the estimators. In Section 3 , we tried to find them using the adaptive tests proposed by Tonaki et al. (2020). When using those tests, it is necessary to check whether the consistency holds. However, the consistency of the tests in Case B was mentioned, but not in Case A. Thus, in this section, we discuss the consistency of the following tests $\mathcal{T}_{n}^{\alpha}, \mathcal{T}_{1, n}^{\beta}$ and $\mathcal{T}_{2, n}^{\beta}$ in Case A.

$$
\begin{aligned}
\mathcal{T}_{n}^{\alpha}=\frac{1}{\sqrt{2 d n}} \max _{1 \leq k \leq n}\left|\sum_{i=1}^{k} \hat{\eta}_{i}-\frac{k}{n} \sum_{i=1}^{n} \hat{\eta}_{i}\right|, \quad \hat{\eta}_{i}=\operatorname{tr}\left(A^{-1}\left(X_{t_{i-1}}, \hat{\alpha}\right) \frac{\left(\Delta X_{i}\right)^{\otimes 2}}{h}\right), \\
\mathcal{T}_{1, n}^{\beta}=\frac{1}{\sqrt{d T}} \max _{1 \leq k \leq n}\left|\sum_{i=1}^{k} \hat{\xi}_{i}-\frac{k}{n} \sum_{i=1}^{n} \hat{\xi}_{i}\right|, \quad \hat{\xi}_{i}=1_{d}^{\top} a^{-1}\left(X_{t_{i-1}}, \hat{\alpha}\right)\left(X_{t_{i}}-X_{t_{i-1}}-h b\left(X_{t_{i-1}}, \hat{\beta}\right)\right), \\
\mathcal{T}_{2, n}^{\beta}=\frac{1}{\sqrt{T}} \max _{1 \leq k \leq n}\left\|\mathcal{I}_{n}^{-1 / 2}\left(\sum_{i=1}^{k} \hat{\zeta}_{i}-\frac{k}{n} \sum_{i=1}^{n} \hat{\zeta}_{i}\right)\right\|, \\
\hat{\zeta}_{i}=\partial_{\beta} b\left(X_{t_{i-1}}, \hat{\beta}\right)^{\top} A^{-1}\left(X_{t_{i-1}}, \hat{\alpha}\right)\left(X_{t_{i}}-X_{t_{i-1}}-h b\left(X_{t_{i-1}}, \hat{\beta}\right)\right), \\
\mathcal{I}_{n}=\frac{1}{n} \sum_{i=1}^{n} \partial_{\beta} b\left(X_{t_{i-1}}, \hat{\beta}\right)^{\top} A^{-1}\left(X_{t_{i-1}}, \hat{\alpha}\right) \partial_{\beta} b\left(X_{t_{i-1}}, \hat{\beta}\right) .
\end{aligned}
$$

First, we consider the power of the test for the diffusion parameter $\alpha$, that is, the following hypothesis testing problem: $H_{0}^{\alpha}: \alpha^{*}$ does not change over $0 \leq t \leq T, H_{1}^{\alpha}$ : There exists $\tau_{*}^{\alpha} \in(0,1)$ such that

$$
\alpha^{*}= \begin{cases}\alpha_{1}^{*}, & t \in\left[0, \tau_{*}^{\alpha} T\right), \\ \alpha_{2}^{*}, & t \in\left[\tau_{*}^{\alpha} T, T\right],\end{cases}
$$

where $\alpha_{1}^{*}$ and $\alpha_{2}^{*}$ depend on $n$, and $\alpha_{1}^{*} \neq \alpha_{2}^{*}$. Let $\vartheta_{\alpha}=\left|\alpha_{1}^{*}-\alpha_{2}^{*}\right|$. Now, we assume the following conditions:

[E1] Under $H_{1}^{\alpha}, \vartheta_{\alpha} \longrightarrow 0$ and $n \vartheta_{\alpha}^{2} \longrightarrow \infty$ as $n \rightarrow \infty$, and there exist $\alpha_{0} \in \operatorname{Int} \Theta_{A}$ and $c_{k} \in \mathbb{R}^{p}$ such that $\vartheta_{\alpha}^{-1}\left(\alpha_{k}^{*}-\alpha_{0}\right) \longrightarrow c_{k}$ for $k=1,2$.

[E2] Under $H_{1}^{\alpha}$, there exists an estimator $\hat{\alpha}$ of $\alpha_{0}$ such that $\vartheta_{\alpha}^{-1}\left(\hat{\alpha}-\alpha_{0}\right)=O_{p}(1)$.

[E3] $\int_{\mathbb{R}^{d}}\left[\operatorname{tr}\left(A^{-1} \partial_{\alpha^{\ell}} A\left(x, \alpha_{0}\right)\right)\right]_{\ell} \mathrm{d} \mu_{\alpha_{0}}(x)\left(c_{1}-c_{2}\right) \neq 0$ under $H_{1}^{\alpha}$.

Remark 5 1-dimensional Ornstein-Ulenbeck process and hyperbolic diffusion model are examples of models which satisfy [E2] and [E3]. See Section 5.2 for some discussion of estimator $\hat{\alpha}$ that satisfies [E2]. See Section 5.3 for models that satisfy [E3].

Let $\epsilon \in(0,1)$ and $w_{k}(\epsilon)$ denote the upper- $\epsilon$ point of $\sup _{0 \leq s \leq 1}\left\|\boldsymbol{B}_{k}^{0}(s)\right\|$, that is, $P\left(\sup _{0 \leq s \leq 1}\left\|\boldsymbol{B}_{k}^{0}(s)\right\|>w_{k}(\epsilon)\right)=\epsilon$. 
Proposition 1 Assume [C1]-[C5], [E1]-[E3]. Then, under $H_{1}^{\alpha}$,

$$
P\left(\mathcal{T}_{n}^{\alpha}>w_{1}(\epsilon)\right) \longrightarrow 1,
$$

that is, the test $\mathcal{T}_{n}^{\alpha}$ is consistent.

In the following, we assume that $\alpha^{*}$ does not change over $0 \leq t \leq T$. We consider the power of the test for the drift parameter $\beta$, that is, the following hypothesis testing problem: $H_{0}^{\beta}: \beta^{*}$ does not change over $0 \leq t \leq T, H_{1}^{\beta}$ : There exists $\tau_{*}^{\beta} \in(0,1)$ such that

$$
\beta^{*}= \begin{cases}\beta_{1}^{*}, & t \in\left[0, \tau_{*}^{\beta} T\right), \\ \beta_{2}^{*}, & t \in\left[\tau_{*}^{\beta} T, T\right],\end{cases}
$$

where $\beta_{1}^{*}$ and $\beta_{2}^{*}$ depend on $n$, and $\beta_{1}^{*} \neq \beta_{2}^{*}$. Let $\vartheta_{\beta}=\left|\beta_{1}^{*}-\beta_{2}^{*}\right|$. Now, we assume the following conditions:

[F1] Under $H_{1}^{\beta}, \vartheta_{\beta} \longrightarrow 0$ and $T \vartheta_{\beta}^{2} \longrightarrow \infty$ as $n \rightarrow \infty$, and there exist $\beta_{0} \in \operatorname{Int} \Theta_{B}$ and $d_{k} \in \mathbb{R}^{q}$ such that $\vartheta_{\beta}^{-1}\left(\beta_{k}^{*}-\beta_{0}\right) \longrightarrow d_{k}$ for $k=1,2$.

[F2] Under $H_{1}^{\beta}$, there exist $\bar{\beta}^{*}$ with $\bar{\beta}^{*}-\beta_{0}=O\left(\vartheta_{\beta}\right)$ and estimators $\hat{\alpha}, \hat{\beta}$ such that

$$
\sqrt{n}\left(\hat{\alpha}-\alpha^{*}\right)=O_{p}(1), \quad \sqrt{T}\left(\hat{\beta}-\bar{\beta}^{*}\right)=O_{p}(1) .
$$

[F3] $\int_{\mathbb{R}^{d}} 1_{d}^{\top} a^{-1}\left(x, \alpha^{*}\right) \partial_{\beta} b\left(x, \beta_{0}\right) \mathrm{d} \mu_{\left(\alpha^{*}, \beta_{0}\right)}(x)\left(d_{1}-d_{2}\right) \neq 0$ under $H_{1}^{\beta}$.

Remark 6 See Kessler $(1995,1997)$, Uchida and Yoshida (2011, 2012), Tonaki et al. (2020) for how to construct the estimators $\hat{\alpha}$ and $\hat{\beta}$ that satisfy [F2]. See Section 5.4 and 5.5 for models that satisfy [F3].

Proposition 2 Assume [C1]-[C5], [F1]-[F3]. Then, under $H_{1}^{\beta}$,

$$
P\left(\mathcal{T}_{1, n}^{\beta}>w_{1}(\epsilon)\right) \longrightarrow 1 .
$$

We additionally assume the following condition:

[F4] There exists an integer $M \geq 3$ such that $h^{-1 / 2} \vartheta_{\beta}^{M-1} \longrightarrow 0$ and $b \in C_{\uparrow}^{4, M}\left(\mathbb{R}^{d} \times \Theta_{B}\right)$.

Proposition 3 Assume [C1]-[C5], [F1], [F2] and [F4]. Then, under $H_{1}^{\beta}$,

$$
P\left(\mathcal{T}_{2, n}^{\beta}>w_{q}(\epsilon)\right) \longrightarrow 1 \text {. }
$$

Remark 7 For the consistency of the test $\mathcal{T}_{2, n}^{\beta}$, only [F1], [F2] and [F4] are sufficient. This is because

[F3'] $\int_{\mathbb{R}^{d}} \partial_{\beta} b\left(x, \beta_{0}\right)^{\top} A^{-1}\left(x, \alpha^{*}\right) \partial_{\beta} b\left(x, \beta_{0}\right) \mathrm{d} \mu_{\beta_{0}}(x)\left(d_{1}-d_{2}\right) \neq 0$,

which corresponds to [F3], is always valid since $\int_{\mathbb{R}^{d}} \partial_{\beta} b\left(x, \beta_{0}\right)^{\mathrm{T}} A^{-1}\left(x, \alpha^{*}\right) \partial_{\beta} b\left(x, \beta_{0}\right) \mathrm{d} \mu_{\beta_{0}}(x)$ is regular and

$$
\left|d_{1}-d_{2}\right|=\lim _{n \rightarrow \infty}\left|\vartheta_{\beta}^{-1}\left(\beta_{1}^{*}-\beta_{0}\right)-\vartheta_{\beta}^{-1}\left(\beta_{2}^{*}-\beta_{0}\right)\right|=\lim _{n \rightarrow \infty}\left|\vartheta_{\beta}^{-1}\left(\beta_{1}^{*}-\beta_{2}^{*}\right)\right|=1 \neq 0 .
$$

\section{EXAMPLES}

5.1. Sufficient condition of assumptions $[\mathbf{A 2}-\mathbf{I}]$ and $[\mathbf{A 2}-\mathbf{I I}]$. A process $\left\{X_{t}\right\}_{t \geq 0}$ with a single change point can be expressed as follows. There exists a process $\left\{\tilde{X}_{t}\right\}_{t \geq 0}$ such that $X_{t}^{1}=\tilde{X}_{t}\left(\theta_{1}^{*}\right), X_{0}^{1}=x_{0}^{1}$, $X_{t}^{2}=\tilde{X}_{t}\left(\theta_{2}^{*}\right), X_{0}^{2}=x_{0}^{2}, X_{\tau T}^{1}=X_{\tau T}^{2}$ and

$$
X_{t}= \begin{cases}X_{t}^{1}, & 0 \leq t<\tau T, \\ X_{t}^{2}, & \tau T \leq t \leq T .\end{cases}
$$

If $\left\{X_{t}^{2}\right\}_{t \geq 0}$ is stationary and $\theta_{2}^{*} \longrightarrow \theta_{0}$, then we have, for $f \in C_{\uparrow}^{1}\left(\mathbb{R}^{d}\right)$,

$$
\begin{aligned}
& \max _{\left[n^{1 / r}\right] \leq k \leq n}\left|\frac{1}{k} \sum_{i=[n \tau]+1}^{[n \tau]+k} f\left(X_{t_{i-1}}\right)-\int_{\mathbb{R}^{d}} f(x) \mathrm{d} \mu_{\theta_{0}}(x)\right| \\
& =\max _{\left[n^{1 / r}\right] \leq k \leq n}\left|\frac{1}{k} \sum_{i=[n \tau]+1}^{[n \tau]+k} f\left(X_{t_{i-1}}^{2}\right)-\int_{\mathbb{R}^{d}} f(x) \mathrm{d} \mu_{\theta_{0}}(x)\right|
\end{aligned}
$$




$$
\stackrel{d}{=} \max _{\left[n^{1 / r}\right] \leq k \leq n}\left|\frac{1}{k} \sum_{i=1}^{k} f\left(X_{t_{i-1}}^{2}\right)-\int_{\mathbb{R}^{d}} f(x) \mathrm{d} \mu_{\theta_{0}}(x)\right| \stackrel{p}{\longrightarrow} 0
$$

and $[\mathbf{A 2} 2-\mathbf{I}]$ or $[\mathbf{A} 2-\mathbf{I I}]$ holds.

5.2. Model that satisfies the assumption [E2]. As an example of the model that satisfies [E2], we consider the $d$-dimensional diffusion process

$$
X_{t}=\left\{\begin{array}{l}
X_{0}+\int_{0}^{t} b\left(X_{s}, \beta\right) \mathrm{d} s+\int_{0}^{t} \sigma\left(X_{s}\right) \delta\left(\boldsymbol{\alpha}_{1}^{*}\right) \mathrm{d} W_{s}, \quad t \in\left[0, \tau_{*}^{\alpha} T\right) \\
X_{\tau_{*}^{\alpha} T}+\int_{\tau_{*}^{\alpha} T}^{t} b\left(X_{s}, \beta\right) \mathrm{d} s+\int_{\tau_{*}^{\alpha} T}^{t} \sigma\left(X_{s}\right) \delta\left(\boldsymbol{\alpha}_{2}^{*}\right) \mathrm{d} W_{s}, \quad t \in\left[\tau_{*}^{\alpha} T, T\right]
\end{array}\right.
$$

where $\sigma: \mathbb{R}^{d} \longrightarrow \mathbb{R}^{d} \otimes \mathbb{R}^{d}, \delta(\boldsymbol{\alpha})=\operatorname{diag}\left(\alpha_{1}, \ldots, \alpha_{d}\right), \boldsymbol{\alpha}=\left(\alpha_{1}, \ldots, \alpha_{d}\right)^{\top}, \alpha_{1}, \ldots, \alpha_{d}>0$. The true value of the parameters are

$$
\boldsymbol{\alpha}_{1}^{*}=\left(\begin{array}{c}
\alpha_{1,1}^{*} \\
\vdots \\
\alpha_{1, d}^{*}
\end{array}\right), \boldsymbol{\alpha}_{2}^{*}=\left(\begin{array}{c}
\alpha_{2,1}^{*} \\
\vdots \\
\alpha_{2, d}^{*}
\end{array}\right)
$$

which convergence to $\boldsymbol{\alpha}_{0}=\left(\alpha_{0,1}, \ldots, \alpha_{0, d}\right)^{\top}$. We define

$$
U_{n}^{(1)}(\boldsymbol{\alpha})=\sum_{i=1}^{n}\left(\operatorname{tr}\left(A_{i-1}^{-1}(\boldsymbol{\alpha}) \frac{\left(\Delta X_{i}\right)^{\otimes 2}}{h}\right)+\log \operatorname{det} A_{i-1}(\boldsymbol{\alpha})\right)
$$

and set the estimator $\hat{\boldsymbol{\alpha}}=\operatorname{arginf}_{\boldsymbol{\alpha}} U_{n}^{(1)}(\boldsymbol{\alpha})$. Then, we have

$$
\vartheta_{\alpha}^{-1}\left(\hat{\boldsymbol{\alpha}}-\boldsymbol{\alpha}_{0}\right)=O_{p}(1) \text {. }
$$

Proof of 5.1. We see

$$
\partial_{\alpha_{j}} U_{n}^{(1)}(\boldsymbol{\alpha})=\frac{2}{\alpha_{j}}\left(\frac{-1}{\alpha_{j}^{2}} \sum_{i=1}^{n} \operatorname{tr}\left(\left[\sigma\left(X_{t_{i-1}}\right)^{\top}\right]^{-1} \delta\left(\mathrm{e}_{j}\right) \sigma\left(X_{t_{i-1}}\right)^{-1} \frac{\left(\Delta X_{i}\right)^{\otimes 2}}{h}\right)+n\right),
$$

where $\mathrm{e}_{j}=(0, \ldots, 1, \ldots, 0)^{\mathrm{\top}}$. Then, we have

$$
\hat{\alpha}_{j}=\sqrt{\frac{1}{n} \sum_{i=1}^{n} \operatorname{tr}\left(\left[\sigma\left(X_{t_{i-1}}\right)^{\top}\right]^{-1} \delta\left(\mathrm{e}_{j}\right) \sigma\left(X_{t_{i-1}}\right)^{-1} \frac{\left(\Delta X_{i}\right)^{\otimes 2}}{h}\right)}
$$

On the other hand, we define

$$
\begin{aligned}
& \underline{U}_{n}^{(1)}(\boldsymbol{\alpha})=\sum_{i=1}^{\left[n \tau_{*}^{\alpha}\right]}\left(\operatorname{tr}\left(A_{i-1}^{-1}(\boldsymbol{\alpha}) \frac{\left(\Delta X_{i}\right)^{\otimes 2}}{h}\right)+\log \operatorname{det} A_{i-1}(\boldsymbol{\alpha})\right), \\
& \bar{U}_{n}^{(1)}(\boldsymbol{\alpha})=\sum_{i=\left[n \tau_{*}^{\alpha}\right]+1}^{n}\left(\operatorname{tr}\left(A_{i-1}^{-1}(\boldsymbol{\alpha}) \frac{\left(\Delta X_{i}\right)^{\otimes 2}}{h}\right)+\log \operatorname{det} A_{i-1}(\boldsymbol{\alpha})\right)
\end{aligned}
$$

and set $\hat{\boldsymbol{\alpha}}_{1}=\operatorname{arginf}_{\boldsymbol{\alpha}} \underline{U}_{n}^{(1)}(\boldsymbol{\alpha}), \hat{\boldsymbol{\alpha}}_{2}=\operatorname{arginf}_{\boldsymbol{\alpha}} \bar{U}_{n}^{(1)}(\boldsymbol{\alpha})$. In the same way, we have

$$
\begin{aligned}
& \hat{\alpha}_{1, j}=\sqrt{\frac{1}{\left[n \tau_{*}^{\alpha}\right]} \sum_{i=1}^{\left[n \tau_{*}^{\alpha}\right]} \operatorname{tr}\left(\left[\sigma\left(X_{t_{i-1}}\right)^{\top}\right]^{-1} \delta\left(\mathrm{e}_{j}\right) \sigma\left(X_{t_{i-1}}\right)^{-1} \frac{\left(\Delta X_{i}\right)^{\otimes 2}}{h}\right)}, \\
& \hat{\alpha}_{2, j}=\sqrt{\frac{1}{n-\left[n \tau_{*}^{\alpha}\right]} \sum_{i=\left[n \tau_{*}^{\alpha}\right]+1}^{n} \operatorname{tr}\left(\left[\sigma\left(X_{t_{i-1}}\right)^{\top}\right]^{-1} \delta\left(\mathrm{e}_{j}\right) \sigma\left(X_{t_{i-1}}\right)^{-1} \frac{\left(\Delta X_{i}\right)^{\otimes 2}}{h}\right)} .
\end{aligned}
$$

Noting that $\vartheta_{\alpha}^{-1}\left(\hat{\boldsymbol{\alpha}}_{k}-\boldsymbol{\alpha}_{0}\right)=O_{p}(1)$ and

$$
\hat{\alpha}_{j}=\sqrt{\frac{\left[n \tau_{*}^{\alpha}\right]}{n} \hat{\alpha}_{1, j}^{2}+\frac{n-\left[n \tau_{*}^{\alpha}\right]}{n} \hat{\alpha}_{2, j}^{2}},
$$


we obtain

$$
\begin{aligned}
\left\|\hat{\boldsymbol{\alpha}}-\boldsymbol{\alpha}_{0}\right\| \leq \sum_{j=1}^{d}\left|\hat{\alpha}_{j}-\alpha_{0, j}\right| & =\sum_{j=1}^{d} \frac{\left|\hat{\alpha}_{j}^{2}-\alpha_{0, j}^{2}\right|}{\left|\hat{\alpha}_{j}+\alpha_{0, j}\right|} \\
& =\sum_{j=1}^{d} \frac{1}{\left|\hat{\alpha}_{j}+\alpha_{0, j}\right|}\left|\frac{\left[n \tau_{*}^{\alpha}\right]}{n}\left(\hat{\alpha}_{1, j}^{2}-\alpha_{0, j}^{2}\right)+\frac{n-\left[n \tau_{*}^{\alpha}\right]}{n}\left(\hat{\alpha}_{2, j}^{2}-\alpha_{0, j}^{2}\right)\right| \\
& \leq \sum_{j=1}^{d} \frac{1}{\left|\hat{\alpha}_{j}+\alpha_{0, j}\right|}\left(\left|\hat{\alpha}_{1, j}-\alpha_{0, j}\right|\left|\hat{\alpha}_{1, j}+\alpha_{0, j}\right|+\left|\hat{\alpha}_{2, j}-\alpha_{0, j}\right|\left|\hat{\alpha}_{2, j}+\alpha_{0, j}\right|\right) \\
& \leq \sum_{j=1}^{d} \vartheta_{\alpha}\left(\frac{\left|\hat{\alpha}_{1, j}+\alpha_{0, j}\right|}{\left|\hat{\alpha}_{j}+\alpha_{0, j}\right|} \vartheta_{\alpha}^{-1}\left|\hat{\alpha}_{1, j}-\alpha_{0, j}\right|+\frac{\left|\hat{\alpha}_{2, j}+\alpha_{0, j}\right|}{\left|\hat{\alpha}_{j}+\alpha_{0, j}\right|} \vartheta_{\alpha}^{-1}\left|\hat{\alpha}_{2, j}-\alpha_{0, j}\right|\right) .
\end{aligned}
$$

From $\hat{\boldsymbol{\alpha}}_{k} \stackrel{p}{\longrightarrow} \boldsymbol{\alpha}_{0}$ and $\hat{\boldsymbol{\alpha}} \stackrel{p}{\longrightarrow} \boldsymbol{\alpha}_{0}$, we have $\frac{\left|\hat{\alpha}_{k, j}+\alpha_{0, j}\right|}{\left|\hat{\alpha}_{j}+\alpha_{0, j}\right|}=O_{p}(1)$ and $\vartheta_{\alpha}^{-1}\left(\hat{\boldsymbol{\alpha}}-\boldsymbol{\alpha}_{0}\right)=O_{p}(1)$.

From the above, 1-dimensional Ornstein-Uhlenbeck process and hyperbolic diffusion model are models which satisfy [E2] because the diffusion coefficient is $a(x, \alpha)=\alpha$.

5.3. Model that satisfies the assumption [E3] and model that does not. First, as an example of a model that satisfies [E3], we consider the $d$-dimensional diffusion process with the diffusion coefficient

$$
a(x, \boldsymbol{\alpha})=\sigma(x) \operatorname{diag}\left(\alpha_{1}, \ldots, \alpha_{d}\right),
$$

where $\sigma: \mathbb{R}^{d} \longrightarrow \mathbb{R}^{d} \otimes \mathbb{R}^{d}, \boldsymbol{\alpha}=\left(\alpha_{1}, \ldots, \alpha_{d}\right)^{\top}, \alpha_{1}, \ldots, \alpha_{d}>0$. The true value of the parameters are $\boldsymbol{\alpha}_{1}^{*}=$ $\boldsymbol{\alpha}_{0}+\vartheta_{\alpha} \boldsymbol{c}_{1}$ and $\boldsymbol{\alpha}_{2}^{*}=\boldsymbol{\alpha}_{0}+\vartheta_{\alpha} \boldsymbol{c}_{2}$, where $\boldsymbol{\alpha}_{0}=\left(\alpha_{0,1}, \ldots, \alpha_{0,3}\right)^{\top}, \boldsymbol{c}_{1}=\left(c_{1,1}, \ldots, c_{1, d}\right)^{\top}, \boldsymbol{c}_{2}=\left(c_{2,1}, \ldots, c_{2, d}\right)^{\top}$. Now we have

$$
\begin{aligned}
& A(x, \boldsymbol{\alpha})=\sigma(x) \operatorname{diag}\left(\alpha_{1}^{2}, \ldots, \alpha_{d}^{2}\right) \sigma(x)^{\top}, \\
& A^{-1}(x, \boldsymbol{\alpha})=\left[\sigma(x)^{\top}\right]^{-1} \operatorname{diag}\left(1 / \alpha_{1}^{2}, \ldots, 1 / \alpha_{d}^{2}\right) \sigma(x)^{-1}, \\
& \partial_{\alpha_{j}} A(x, \boldsymbol{\alpha})=\sigma(x) \operatorname{diag}\left(0, \ldots, 2 \alpha_{j}, \ldots, 0\right) \sigma(x)^{\top}, \\
& \operatorname{tr}\left(A^{-1} \partial_{\alpha_{j}} A(x, \boldsymbol{\alpha})\right)=\operatorname{tr}\left(\left[\sigma(x)^{\top}\right]^{-1} \operatorname{diag}\left(0, \ldots, 2 / \alpha_{j}, \ldots, 0\right) \sigma(x)^{\top}\right)=\frac{2}{\alpha_{j}}, \\
& \int_{\mathbb{R}^{d}}\left[\operatorname{tr}\left(A^{-1} \partial_{\alpha^{\ell}} A\left(x, \boldsymbol{\alpha}_{0}\right)\right)\right]_{\ell} \mathrm{d} \mu_{\alpha_{0}}(x)\left(\boldsymbol{c}_{1}-\boldsymbol{c}_{2}\right)=\sum_{j=1}^{d} \frac{2\left(c_{1, j}-c_{2, j}\right)}{\alpha_{0, j}} .
\end{aligned}
$$

Therefore if

$$
\sum_{j=1}^{d} \frac{c_{1, j}-c_{2, j}}{\alpha_{j}} \neq 0,
$$

then [E3] holds. Especially, we have (5.2) if any of the following cases:

(1) $c_{1, j}-c_{2, j} \geq 0$ for all $1 \leq j \leq d$, and $c_{1, j}-c_{2, j}>0$ for some $1 \leq j \leq d$.

(2) $c_{1, j}-c_{2, j} \leq 0$ for all $1 \leq j \leq d$, and $c_{1, j}-c_{2, j}<0$ for some $1 \leq j \leq d$.

That is, [E3] holds when only $\alpha_{j}(1 \leq j \leq d)$ changes. From the above, 1-dimensional Ornstein-Uhlenbeck process and hyperbolic diffusion model are models which satisfy [E3] because the diffusion coefficient is $a(x, \alpha)=\alpha$.

Next, as an example of a model that does not satisfy [E3], we consider the 2-dimensional diffusion process with diffusion coefficient

$$
a(x, \boldsymbol{\alpha})=\left(\begin{array}{cc}
\alpha_{1} & \alpha_{2} \\
0 & \alpha_{3}
\end{array}\right), \quad\left(\alpha_{1}, \alpha_{3}>0\right)
$$


The true value of the parameters are

$$
\boldsymbol{\alpha}_{0}=\left(\begin{array}{c}
\alpha_{0,1} \\
\alpha_{0,2} \\
\alpha_{0,3}
\end{array}\right), \boldsymbol{\alpha}_{1}^{*}=\boldsymbol{\alpha}_{0}+\vartheta_{\alpha}\left(\begin{array}{c}
c_{1,1} \\
c_{1,2} \\
c_{1,3}
\end{array}\right), \boldsymbol{\alpha}_{2}^{*}=\boldsymbol{\alpha}_{0}+\vartheta_{\alpha}\left(\begin{array}{c}
c_{2,1} \\
c_{2,2} \\
c_{2,3}
\end{array}\right) .
$$

Now we have

$$
\begin{gathered}
A(x, \boldsymbol{\alpha})=\left(\begin{array}{cc}
\alpha_{1} & \alpha_{2} \\
0 & \alpha_{3}
\end{array}\right)\left(\begin{array}{cc}
\alpha_{1} & 0 \\
\alpha_{2} & \alpha_{3}
\end{array}\right)=\left(\begin{array}{cc}
\alpha_{1}^{2}+\alpha_{2}^{2} & \alpha_{2} \alpha_{3} \\
\alpha_{2} \alpha_{3} & \alpha_{3}^{2}
\end{array}\right), \\
A^{-1}(x, \boldsymbol{\alpha})=\frac{1}{\alpha_{1}^{2} \alpha_{3}^{2}}\left(\begin{array}{cc}
\alpha_{3}^{2} & -\alpha_{2} \alpha_{3} \\
-\alpha_{2} \alpha_{3} & \alpha_{1}^{2}+\alpha_{2}^{2}
\end{array}\right), \\
\partial_{\alpha_{1}} A(x, \boldsymbol{\alpha})=\left(\begin{array}{cc}
2 \alpha_{1} & 0 \\
0 & 0
\end{array}\right), \partial_{\alpha_{2}} A(x, \boldsymbol{\alpha})=\left(\begin{array}{cc}
2 \alpha_{2} & \alpha_{3} \\
\alpha_{3} & 0
\end{array}\right), \partial_{\alpha_{3}} A(x, \boldsymbol{\alpha})=\left(\begin{array}{cc}
0 & \alpha_{2} \\
\alpha_{2} & 2 \alpha_{3}
\end{array}\right), \\
\operatorname{tr}\left(A^{-1} \partial_{\alpha_{1}} A(x, \boldsymbol{\alpha})\right)=\frac{2 \alpha_{1} \alpha_{3}^{2}}{\alpha_{1}^{2} \alpha_{3}^{2}}=\frac{2}{\alpha_{1}}, \\
\operatorname{tr}\left(A^{-1} \partial_{\alpha_{2}} A(x, \boldsymbol{\alpha})\right)=\frac{\alpha_{2} \alpha_{3}^{2}-\alpha_{2} \alpha_{3}^{2}}{\alpha_{1}^{2} \alpha_{3}^{2}}=0, \\
\operatorname{tr}\left(A^{-1} \partial_{\alpha_{3}} A(x, \boldsymbol{\alpha})\right)=\frac{-\alpha_{2}^{2} \alpha_{3}+2 \alpha_{1}^{2} \alpha_{3}+\alpha_{2}^{2} \alpha_{3}}{\alpha_{1}^{2} \alpha_{3}^{2}}=\frac{2}{\alpha_{3}}, \\
\int_{\mathbb{R}^{2}}\left(\frac{2}{\alpha_{0,1}}, 0, \frac{2}{\alpha_{0,3}}\right) \mathrm{d} \mu_{\boldsymbol{\alpha}_{0}}(x)\left(\begin{array}{c}
c_{1,1}-c_{2,1} \\
c_{1,2}-c_{2,2} \\
c_{1,3}-c_{2,3}
\end{array}\right)=\frac{2\left(c_{1,1}-c_{2,1}\right)}{\alpha_{0,1}}+\frac{2\left(c_{1,3}-c_{2,3}\right)}{\alpha_{0,3}} .
\end{gathered}
$$

Therefore, (5.3) does not equal 0 in the following cases:

(1) $c_{1, j}-c_{2, j} \geq 0$ for all $j=1,3$, and $c_{1, j}-c_{2, j}>0$ for some $j=1,3$.

(2) $c_{1, j}-c_{2, j} \leq 0$ for all $j=1,3$, and $c_{1, j}-c_{2, j}<0$ for some $j=1,3$.

However (5.3) equals 0 when $c_{1,1}-c_{2,1}=c_{1,3}-c_{2,3}=0$, hence when only $\alpha_{2}$ changes, [E3] does not hold.

5.4. Ornstein-Uhlenbeck process. We consider the 1-dimensional Ornstein-Uhlenbeck process

$$
\mathrm{d} X_{t}=-\beta\left(X_{t}-\gamma\right) \mathrm{d} t+\alpha \mathrm{d} W_{t}, \quad X_{0}=x_{0} . \quad(\alpha, \beta>0, \gamma \in \mathbb{R}) .
$$

In this subsection, we refer to the consistency of tests $\mathcal{T}_{n}^{\alpha}$ and $\mathcal{T}_{1, n}^{\beta}$ in Case A.

First, let us consider the consistency of the test $\mathcal{T}_{n}^{\alpha}$ in Case A. Thus, we consider the following stochastic differential equation

$$
X_{t}=\left\{\begin{array}{l}
X_{0}-\int_{0}^{t} \beta\left(X_{s}-\gamma\right) \mathrm{d} s+\alpha_{1}^{*} W_{t}, \quad t \in\left[0, \tau_{*}^{\alpha} T\right), \\
X_{\tau_{*}^{\alpha} T}-\int_{\tau_{*}^{\alpha} T}^{t} \beta\left(X_{s}-\gamma\right) \mathrm{d} s+\alpha_{2}^{*}\left(W_{t}-W_{\tau_{*}^{\alpha} T}\right), \quad t \in\left[\tau_{*}^{\alpha} T, T\right],
\end{array}\right.
$$

where $\alpha_{1}^{*}=\alpha_{0}+\vartheta_{\alpha} c_{1}$ and $\alpha_{2}^{*}=\alpha_{0}+\vartheta_{\alpha} c_{2}$, which hold [E1]. Further, from Section 5.2 and 5.3. [E2] and [E3] hold. Therefore the test $\mathcal{T}_{n}^{\alpha}$ is consistent by Proposition 1.

Next, we investigate the consistency of the test $\mathcal{T}_{1, n}^{\beta}$ in Case A. Thus, we consider the following stochastic differential equation

$$
X_{t}=\left\{\begin{array}{l}
X_{0}-\int_{0}^{t} \beta_{1}^{*}\left(X_{s}-\gamma_{1}^{*}\right) \mathrm{d} s+\alpha^{*} W_{t}, \quad t \in\left[0, \tau_{*}^{\beta} T\right), \\
X_{\tau_{*}^{\beta} T}-\int_{\tau_{*}^{\beta} T}^{t} \beta_{2}^{*}\left(X_{s}-\gamma_{2}^{*}\right) \mathrm{d} s+\alpha^{*}\left(W_{t}-W_{\tau_{*}^{\beta} T}\right), \quad t \in\left[\tau_{*}^{\beta} T, T\right],
\end{array}\right.
$$

where

$$
\boldsymbol{\beta}_{0}=\left(\begin{array}{c}
\beta_{0} \\
\gamma_{0}
\end{array}\right), \boldsymbol{d}_{k}=\left(\begin{array}{c}
d_{k, 1} \\
d_{k, 2}
\end{array}\right), \boldsymbol{\beta}_{k}^{*}=\left(\begin{array}{c}
\beta_{k}^{*} \\
\gamma_{k}^{*}
\end{array}\right)=\boldsymbol{\beta}_{0}+\vartheta_{\beta} \boldsymbol{d}_{k}
$$


which hold [F1]. Further,

$$
\int_{\mathbb{R}} \frac{1}{\alpha^{*}}\left(-x+\gamma_{0}, \beta_{0}\right) \mathrm{d} \mu_{\left(\beta_{0}, \gamma_{0}\right)}(x)\left(\boldsymbol{d}_{1}-\boldsymbol{d}_{2}\right)=\left(0, \frac{\beta_{0}}{\alpha^{*}}\right)\left(\begin{array}{c}
d_{1,1}-d_{2,1} \\
d_{1,2}-d_{2,2}
\end{array}\right)=\frac{\beta_{0}}{\alpha^{*}}\left(d_{1,2}-d_{2,2}\right) .
$$

Therefore, if $d_{1,2}-d_{2,2} \neq 0$, that is, if $\gamma$ changes and $\beta$ does not change, then [F3] holds, and the test $\mathcal{T}_{1, n}^{\beta}$ is consistent by Proposition 2. However, when $\beta$ changes and $\gamma$ does not change, [F3] does not hold.

5.5. Hyperbolic diffusion model. We consider the hyperbolic diffusion model

$$
\mathrm{d} X_{t}=\left(\beta-\frac{\gamma X_{t}}{\sqrt{1+X_{t}^{2}}}\right) \mathrm{d} t+\alpha \mathrm{d} W_{t}, \quad X_{0}=x_{0} . \quad(\alpha>0, \beta \in \mathbb{R}, \gamma>|\beta|) .
$$

In this subsection, we mention the consistency of the tests $\mathcal{T}_{n}^{\alpha}$ and $\mathcal{T}_{1, n}^{\beta}$ in Case $\mathrm{A}$ and the fact that this model is an example in Case B.

Let $b(x, \boldsymbol{\beta})=\beta-\frac{\gamma x}{\sqrt{1+x^{2}}}$ and $a(x, \alpha)=\alpha$. By the same discussion as Ornstein-Uhlenbeck process (Section 5.4, the test $\mathcal{T}_{n}^{\alpha}$ is consistent in Case A. Next, we investigate the consistency of the test $\mathcal{T}_{1, n}^{\beta}$ in Case A. Thus, we consider the following stochastic differential equation

$$
X_{t}=\left\{\begin{array}{l}
X_{0}+\int_{0}^{t}\left(\beta_{1}^{*}-\frac{\gamma_{1}^{*} X_{s}}{\sqrt{1+X_{s}^{2}}}\right) \mathrm{d} s+\alpha^{*} W_{t}, \quad t \in\left[0, \tau_{*}^{\beta} T\right), \\
X_{\tau_{*}^{\beta} T}+\int_{\tau_{*}^{\beta} T}^{t}\left(\beta_{2}^{*}-\frac{\gamma_{2}^{*} X_{s}}{\sqrt{1+X_{s}^{2}}}\right) \mathrm{d} s+\alpha^{*}\left(W_{t}-W_{\tau_{*}^{\beta} T}\right), \quad t \in\left[\tau_{*}^{\beta} T, T\right],
\end{array}\right.
$$

where

$$
\boldsymbol{\beta}_{0}=\left(\begin{array}{c}
\beta_{0} \\
\gamma_{0}
\end{array}\right), \boldsymbol{d}_{k}=\left(\begin{array}{c}
d_{k, 1} \\
d_{k, 2}
\end{array}\right), \boldsymbol{\beta}_{k}^{*}=\left(\begin{array}{c}
\beta_{k}^{*} \\
\gamma_{k}^{*}
\end{array}\right)=\boldsymbol{\beta}_{0}+\vartheta_{\beta} \boldsymbol{d}_{k},
$$

which hold [F1]. The invariant density of the solution in 5.4 is

where

$$
\pi(x)=\frac{m(x)}{M},
$$

$$
m(x)=\exp \left(\frac{2}{\alpha^{2}}\left(\beta x-\gamma \sqrt{1+x^{2}}\right)\right), M=\int_{\mathbb{R}} m(x) \mathrm{d} x
$$

Now, we have

$$
\begin{gathered}
\int_{\mathbb{R}} \partial_{\beta} b(x, \boldsymbol{\beta}) \pi(x) \mathrm{d} x=\int_{\mathbb{R}} \pi(x) \mathrm{d} x=1 \\
\int_{\mathbb{R}} \partial_{\gamma} b(x, \boldsymbol{\beta}) \pi(x) \mathrm{d} x=-\frac{1}{M} \int_{\mathbb{R}} \frac{x}{\sqrt{1+x^{2}}} \exp \left(\frac{2}{\alpha^{2}}\left(\beta x-\gamma \sqrt{1+x^{2}}\right)\right) \mathrm{d} x \\
=\frac{\alpha^{2}}{2 \gamma}\left(\frac{1}{M} \int_{\mathbb{R}} \frac{2}{\alpha^{2}}\left(\beta-\frac{\gamma x}{\sqrt{1+x^{2}}}\right) \exp \left(\frac{2}{\alpha^{2}}\left(\beta x-\gamma \sqrt{1+x^{2}}\right)\right) \mathrm{d} x-\frac{2 \beta}{\alpha^{2}}\right)
\end{gathered}
$$

and

$$
\begin{aligned}
& \int_{\mathbb{R}} \frac{2}{\alpha^{2}}\left(\beta-\frac{\gamma x}{\sqrt{1+x^{2}}}\right) \exp \left(\frac{2}{\alpha^{2}}\left(\beta x-\gamma \sqrt{1+x^{2}}\right)\right) \mathrm{d} x \\
& =\left[\exp \left(\frac{2}{\alpha^{2}}\left(\beta x-\gamma \sqrt{1+x^{2}}\right)\right)\right]_{x=-\infty}^{x=\infty} \\
& =\left[\exp \left(-\frac{2 \gamma}{\alpha^{2}} \sqrt{1+x^{2}}\left(1-\frac{\beta}{\gamma} \frac{x}{\sqrt{1+x^{2}}}\right)\right)\right]_{x=-\infty}^{x=\infty} \\
& =0 .
\end{aligned}
$$

Hence, we have

$$
\int_{\mathbb{R}} \partial_{\gamma} b(x, \boldsymbol{\beta}) \pi(x) \mathrm{d} x=-\frac{\beta}{\gamma} .
$$


From the above, we obtain

$$
\begin{aligned}
\int_{\mathbb{R}} \frac{1}{\alpha^{*}}\left(1,-\frac{\beta_{0}}{\gamma_{0}}\right) \mathrm{d} \mu_{\left(\beta_{0}, \gamma_{0}\right)}(x)\left(\boldsymbol{d}_{1}-\boldsymbol{d}_{2}\right) & =\frac{1}{\alpha^{*}}\left(1,-\frac{\beta_{0}}{\gamma_{0}}\right)\left(\begin{array}{c}
d_{1,1}-d_{2,1} \\
d_{1,2}-d_{2,2}
\end{array}\right) \\
& =\frac{1}{\alpha^{*}}\left(\left(d_{1,1}-d_{2,1}\right)-\frac{\beta_{0}}{\gamma_{0}}\left(d_{1,2}-d_{2,2}\right)\right) .
\end{aligned}
$$

In the following cases, [F3] holds because equation (5.5) does not equal 0:

(1) $\beta$ changes and $\gamma$ does not change,

(2) $\beta_{0} \neq 0, \gamma$ changes and $\beta$ does not change,

(3) $\beta_{0}>0, d_{1,1}-d_{2,1}<0$ (resp. $>0$ ) and $d_{1,2}-d_{2,2}>0$ (resp. $\left.<0\right)$,

(4) $\beta_{0}<0, d_{1,1}-d_{2,1}<0$ (resp. $>0$ ) and $d_{1,2}-d_{2,2}<0$ (resp. $>0$ ).

Finally, in Case B, we confirm that the hyperbolic diffusion model satisfies the assumptions [B1-I], [B2-I], [B1-II] and [B2-II]. Note that it was mentioned in Remark 1 that [B1-I] and [B2-I](a), (b) hold. Thus, in the following, we verify that [B2-I] $(\mathrm{c}),[\mathbf{B 1}-\mathbf{I I}]$ and [B2-II] hold. From the proof of Lemma 1 of Kessler (1997), we can express $Q(x, \theta)=\left.L_{\theta}^{2} f(y \mid x)\right|_{y=x}$, where $f(y \mid x)=(y-x)^{2}$,

$$
\begin{aligned}
& L_{\theta} f(y \mid x)=b(y, \boldsymbol{\beta}) \partial_{y} f(y \mid x)+\frac{A(y, \alpha)}{2} \partial_{y}^{2} f(y \mid x), \\
& L_{\theta}^{2} f(y \mid x)=L_{\theta}\left[L_{\theta} f\right](y \mid x) .
\end{aligned}
$$

The specific calculation is as follows.

$$
\begin{aligned}
L_{\theta} f(y \mid x) & =2 b(y, \boldsymbol{\beta})(y-x)+\alpha^{2}, \\
\partial_{y}\left[L_{\theta} f\right](y \mid x) & =2\left(\partial_{y} b(y, \boldsymbol{\beta})(y-x)+b(y, \boldsymbol{\beta})\right), \\
\partial_{y}^{2}\left[L_{\theta} f\right](y \mid x) & =2\left(\partial_{y}^{2} b(y, \boldsymbol{\beta})(y-x)+2 \partial_{y} b(y, \boldsymbol{\beta})\right), \\
L_{\theta}^{2} f(y \mid x) & =2 b(y, \beta)\left(\partial_{y} b(y, \boldsymbol{\beta})(y-x)+b(y, \boldsymbol{\beta})\right)+\alpha^{2}\left(\partial_{y}^{2} b(y, \boldsymbol{\beta})(y-x)+2 \partial_{y} b(y, \boldsymbol{\beta})\right),
\end{aligned}
$$

and $Q(x, \theta)=2\left(b(x, \boldsymbol{\beta})^{2}+\alpha^{2} \partial_{x} b(x, \boldsymbol{\beta})\right) . b(x, \boldsymbol{\beta})$ and $\partial_{x} b(x, \boldsymbol{\beta})$ are bounded, so we have $\sup _{x, \theta}|Q(x, \theta)|<C$. Thus, [B2-I](c) holds. Moreover, we have

$$
\begin{aligned}
\Gamma^{\beta}\left(x, \alpha, \boldsymbol{\beta}_{1}, \boldsymbol{\beta}_{2}\right) & =\frac{1}{\alpha^{2}}\left[\left(\beta_{1}-\frac{\gamma_{1} x}{\sqrt{1+x^{2}}}\right)-\left(\beta_{2}-\frac{\gamma_{2} x}{\sqrt{1+x^{2}}}\right)\right]^{2} \\
& =\frac{1}{\alpha^{2}}\left(\left(\beta_{1}-\beta_{2}\right)-\left(\gamma_{1}-\gamma_{2}\right) \frac{x}{\sqrt{1+x^{2}}}\right)^{2}
\end{aligned}
$$

where $\boldsymbol{\beta}_{k}=\left(\beta_{k}, \gamma_{k}\right)^{\top}$. Therefore, since $-1<\frac{x}{\sqrt{1+x^{2}}}<1$ for $x \in \mathbb{R}$, we have $\sup _{x}\left|\Gamma^{\beta}\left(x, \alpha^{*}, \boldsymbol{\beta}_{1}^{*}, \boldsymbol{\beta}_{2}^{*}\right)\right|>0$ in the following cases, and [B1-II] holds.

(1) $\gamma_{1}^{*}=\gamma_{2}^{*}$,

(2) $\gamma_{1}^{*} \neq \gamma_{2}^{*}$ and $\beta_{1}^{*}-\beta_{2}^{*}<-\left(\gamma_{1}^{*}-\gamma_{2}^{*}\right)$,

(3) $\gamma_{1}^{*} \neq \gamma_{2}^{*}$ and $\beta_{1}^{*}-\beta_{2}^{*}>\gamma_{1}^{*}-\gamma_{2}^{*}$.

Furthermore, we see, from

$$
\begin{gathered}
\partial_{\alpha} \Gamma^{\beta}\left(x, \alpha, \boldsymbol{\beta}_{1}, \boldsymbol{\beta}_{2}\right)=\frac{-2}{\alpha^{3}}\left(\left(\beta_{1}-\beta_{2}\right)-\left(\gamma_{1}-\gamma_{2}\right) \frac{x}{\sqrt{1+x^{2}}}\right)^{2}, \\
\partial_{\beta_{1}} \Gamma^{\beta}\left(x, \alpha, \boldsymbol{\beta}_{1}, \boldsymbol{\beta}_{2}\right)=\frac{2}{\alpha^{2}}\left(\left(\beta_{1}-\beta_{2}\right)-\left(\gamma_{1}-\gamma_{2}\right) \frac{x}{\sqrt{1+x^{2}}}\right), \\
\partial_{\gamma_{1}} \Gamma^{\beta}\left(x, \alpha, \boldsymbol{\beta}_{1}, \boldsymbol{\beta}_{2}\right)=\frac{-2 x}{\alpha^{2} \sqrt{1+x^{2}}}\left(\left(\beta_{1}-\beta_{2}\right)-\left(\gamma_{1}-\gamma_{2}\right) \frac{x}{\sqrt{1+x^{2}}}\right), \\
\partial_{\beta_{2}} \Gamma^{\beta}\left(x, \alpha, \boldsymbol{\beta}_{1}, \boldsymbol{\beta}_{2}\right)=-\partial_{\beta_{1}} \Gamma^{\beta}\left(x, \alpha, \boldsymbol{\beta}_{1}, \boldsymbol{\beta}_{2}\right), \partial_{\gamma_{2}} \Gamma^{\beta}\left(x, \alpha, \boldsymbol{\beta}_{1}, \boldsymbol{\beta}_{2}\right)=-\partial_{\gamma_{1}} \Gamma^{\beta}\left(x, \alpha, \boldsymbol{\beta}_{1}, \boldsymbol{\beta}_{2}\right), \\
\frac{1}{\alpha^{2}} \partial_{\beta} b(x, \boldsymbol{\beta})\left(b\left(x, \boldsymbol{\beta}_{1}\right)-b\left(x, \boldsymbol{\beta}_{2}\right)\right)^{2}=\frac{1}{\alpha^{2}}\left(\left(\beta_{1}-\beta_{2}\right)-\left(\gamma_{1}-\gamma_{2}\right) \frac{x}{\sqrt{1+x^{2}}}\right), \\
\frac{1}{\alpha^{2}} \partial_{\gamma} b(x, \boldsymbol{\beta})\left(b\left(x, \boldsymbol{\beta}_{1}\right)-b\left(x, \boldsymbol{\beta}_{2}\right)\right)^{2}=\frac{-x}{\alpha^{2} \sqrt{1+x^{2}}}\left(\left(\beta_{1}-\beta_{2}\right)-\left(\gamma_{1}-\gamma_{2}\right) \frac{x}{\sqrt{1+x^{2}}}\right)
\end{gathered}
$$


and the boundedness of $\frac{x}{\sqrt{1+x^{2}}}$, that

$$
\begin{aligned}
& \sup _{x, \alpha, \boldsymbol{\beta}_{k}}\left|\partial_{\left(\alpha, \boldsymbol{\beta}_{1}, \boldsymbol{\beta}_{2}\right)} \Gamma^{\beta}\left(x, \alpha, \boldsymbol{\beta}_{1}, \boldsymbol{\beta}_{2}\right)\right|<C, \\
& \sup _{x, \alpha, \boldsymbol{\beta}_{k}}\left|\frac{1}{\alpha^{2}} \partial_{\boldsymbol{\beta}} b(x, \boldsymbol{\beta})\left(b\left(x, \boldsymbol{\beta}_{1}\right)-b\left(x, \boldsymbol{\beta}_{2}\right)\right)^{2}\right|<C
\end{aligned}
$$

and [B2-II] holds.

\section{Simulations}

6.1. Case A. In this subsection, we consider the 1-dimensional Ornstein-Uhlenbeck process:

$$
\mathrm{d} X_{t}=-\beta\left(X_{t}-\gamma\right) \mathrm{d} t+\alpha \mathrm{d} W_{t}, \quad X_{0}=x_{0},
$$

where $\alpha, \beta>0, \gamma \in \mathbb{R}$.

In order to check the asymptotic behavior of the estimator $\hat{\tau}_{n}^{\alpha}$ in Case A, we consider the following stochastic differential equation

$$
X_{t}=\left\{\begin{array}{l}
X_{0}-\int_{0}^{t} \beta^{*}\left(X_{s}-\gamma^{*}\right) \mathrm{d} s+\alpha_{1}^{*} W_{t}, \quad t \in\left[0, \tau_{*}^{\alpha} T\right), \\
X_{\tau_{*}^{\alpha} T}-\int_{\tau_{*}^{\alpha} T}^{t} \beta^{*}\left(X_{s}-\gamma^{*}\right) \mathrm{d} s+\alpha_{2}^{*}\left(W_{t}-W_{\tau_{*}^{\alpha} T}\right), \quad t \in\left[\tau_{*}^{\alpha} T, T\right],
\end{array}\right.
$$

where $x_{0}=2, \beta^{*}=1, \gamma^{*}=2$ and $\tau_{*}^{\alpha}=0.5$. The number of iteration is 1000 . We set that the sample size of the data $\left\{X_{t_{i}}\right\}_{i=0}^{n}$ is $n=10^{6}$ and $h_{n}=n^{-2 / 3}=10^{-4}$. Note that $T=n h_{n}=n^{1 / 3}=10^{2}, n h_{n}^{2}=n^{-1 / 3}=10^{-2}$, $\vartheta_{\alpha}=n^{-0.35} \approx 0.0079, n \vartheta_{\alpha}^{2}=n^{0.3} \approx 63.1, T \vartheta_{\alpha}=n^{-1 / 60} \approx 0.79, \alpha_{0}=0.1, \alpha_{1}^{*}=\alpha_{0}+n^{-0.35}$ and $\alpha_{2}^{*}=\alpha_{0}=0.1$. The existence of a change point in the intervals $[1 / 4 T, T]$ and $[0,3 / 4 T]$ was investigated using the method of Tonaki et al. (2020). In all 1000 iterations, the change point was detected. Therefore, we estimated $\hat{\alpha}_{1}$ and $\hat{\alpha}_{2}$ with $\underline{\tau}^{\alpha}=1 / 4$ and $\bar{\tau}^{\alpha}=3 / 4$, respectively. The estimates of $\alpha_{1}^{*}, \alpha_{2}^{*}$ and $\tau_{*}^{\alpha}$ are reported in Table 1. Moreover, one has that for $v \in \mathbb{R}$,

$$
\begin{aligned}
e_{\alpha} & =\lim _{n \rightarrow \infty} \vartheta_{\alpha}^{-1}\left(\alpha_{1}^{*}-\alpha_{2}^{*}\right)=1 \\
\mathcal{J}_{\alpha} & =\frac{1}{2} e_{\alpha}^{\top} \int_{\mathbb{R}^{d}} \Xi^{\alpha}\left(x, \alpha_{0}\right) \mathrm{d} \mu_{\alpha_{0}}(x) e_{\alpha}=\frac{2}{\alpha_{0}^{2}} \\
\mathbb{F}(v) & =-2 \mathcal{J}_{\alpha}^{1 / 2} \mathcal{W}(v)+\mathcal{J}_{\alpha}|v|=-2\left(\mathcal{J}_{\alpha}^{1 / 2} \mathcal{W}(v)-\frac{1}{2}\left|\mathcal{J}_{\alpha} v\right|\right) \stackrel{d}{=}-2\left(\mathcal{W}\left(\mathcal{J}_{\alpha} v\right)-\frac{1}{2}\left|\mathcal{J}_{\alpha} v\right|\right) .
\end{aligned}
$$

It follows from Theorem 1 that

$$
n \vartheta_{\alpha}^{2}\left(\hat{\tau}_{n}^{\alpha}-\tau_{*}^{\alpha}\right) \stackrel{d}{\longrightarrow} \underset{v \in \mathbb{R}}{\operatorname{argmin}} \mathbb{F}(v) .
$$

For $v \in \mathbb{R}$, let $G(v)=\mathcal{W}(v)-\frac{1}{2}|v|$ and $\hat{\eta}=\inf \left\{\eta \in \mathbb{R} \mid G(\eta)=\sup _{v \in \mathbb{R}} G(v)\right\}$. For the probability density function of the distribution of $\hat{\eta}$, see Lemma 1.6.3 of Csörgö and Horváth (1997). From Figure 3 , we can see that the distribution of the estimator almost corresponds with the asymptotic distribution in Theorem 1 and the estimators have good performance.

TABle 1. Mean and standard deviation of the estimators under $n=10^{6}, T=100$, $h=10^{-4}, \tau_{*}^{\alpha}=0.5, \alpha_{1}^{*} \approx 0.1079, \alpha_{2}^{*}=0.1$ in Case $\mathrm{A}$.

\begin{tabular}{ccc}
\hline$\hat{\alpha}_{1}$ & $\hat{\alpha}_{2}$ & $\hat{\tau}_{n}^{\alpha}$ \\
\hline 0.10795 & 0.10001 & 0.49999 \\
$(0.00016)$ & $(0.00014)$ & $(0.00041)$ \\
\hline
\end{tabular}



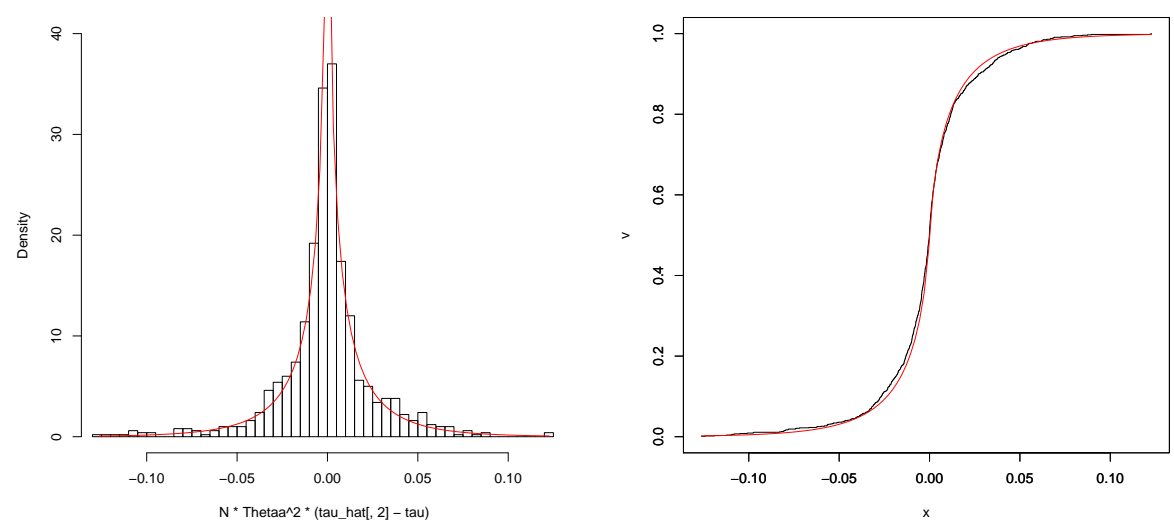

FiguRE 3. The figure on the left is the histogram of $n \vartheta_{\alpha}^{2}\left(\hat{\tau}_{n}^{\alpha}-\tau_{*}^{\alpha}\right)$ (black line) and the theoretical density function (red line). The figure on the right is the empirical distribution function of $n \vartheta_{\alpha}^{2}\left(\hat{\tau}_{n}^{\alpha}-\tau_{*}^{\alpha}\right)$ (black line) and the theoretical distribution function (red line).

For the simulations of the estimator $\hat{\tau}_{n}^{\beta}$ in Case A, we treat the stochastic differential equation as follows.

$$
X_{t}=\left\{\begin{array}{l}
X_{0}-\int_{0}^{t} \beta^{*}\left(X_{s}-\gamma_{1}^{*}\right) \mathrm{d} s+\alpha^{*} W_{t}, \quad t \in\left[0, \tau_{*}^{\beta} T\right), \\
X_{\tau_{*}^{\beta} T}-\int_{\tau_{*}^{\beta} T}^{t} \beta^{*}\left(X_{s}-\gamma_{2}^{*}\right) \mathrm{d} s+\alpha^{*}\left(W_{t}-W_{\tau_{*}^{\beta} T}\right), \quad t \in\left[\tau_{*}^{\beta} T, T\right],
\end{array}\right.
$$

where $x_{0}=5, \alpha^{*}=0.5, \beta^{*}=2.5$ and $\tau_{*}^{\beta}=0.5$. The number of iteration is 1000 . We set that the sample size of the data $\left\{X_{t_{i}}\right\}_{i=0}^{n}$ is $n=10^{6}$ and $h_{n}=n^{-4 / 7} \approx 3.73 \times 10^{-4}$. Note that $T=n h_{n}=n^{3 / 7} \approx 3.73 \times 10^{2}$, $n h_{n}^{2}=n^{-1 / 7} \approx 0.14, \vartheta_{\beta}=n^{-1 / 8} \approx 0.18, T \vartheta_{\beta}^{2}=n^{5 / 28} \approx 11.8, T \vartheta_{\beta}^{4}=n^{-1 / 14} \approx 0.37 \gamma_{1}^{*}=5+\vartheta_{\beta}$ and $\gamma_{2}^{*}=5$. In all iterations, the change point was detected in the intervals $[1 / 4 T, T]$ and $[0,3 / 4 T]$. Therefore, we estimated $\hat{\boldsymbol{\beta}}_{1}$ and $\hat{\boldsymbol{\beta}}_{2}$ with $\underline{\tau}^{\beta}=1 / 4$ and $\bar{\tau}^{\beta}=3 / 4$, respectively. The estimates of $\boldsymbol{\beta}_{1}^{*}, \boldsymbol{\beta}_{2}^{*}$ and $\tau_{*}^{\beta}$ are reported in Table 2. Noting that $\boldsymbol{\beta}_{1}^{*}=\left(\beta^{*}, \gamma_{1}^{*}\right)^{\top}, \boldsymbol{\beta}_{2}^{*}=\left(\beta^{*}, \gamma_{2}^{*}\right)^{\top}, \boldsymbol{\beta}_{0}=\left(\beta^{*}, \gamma_{2}^{*}\right)^{\top}, \mu_{\left(\alpha^{*}, \boldsymbol{\beta}_{0}\right)} \sim N\left(\gamma_{2}^{*}, \frac{\left(\alpha^{*}\right)^{2}}{2 \beta^{*}}\right)$ and

$$
\Xi^{\beta}(x, \alpha, \boldsymbol{\beta})=\frac{1}{\alpha^{2}}\left(\begin{array}{cc}
(x-\gamma)^{2} & -\beta(x-\gamma) \\
-\beta(x-\gamma) & \beta^{2}
\end{array}\right)
$$

we obtain that for $v \in \mathbb{R}$,

$$
\begin{aligned}
e_{\beta} & =\lim _{n \rightarrow \infty} \vartheta_{\beta}^{-1}\left(\boldsymbol{\beta}_{1}^{*}-\boldsymbol{\beta}_{2}^{*}\right)=\left(\begin{array}{c}
0 \\
1
\end{array}\right), \\
\mathcal{J}_{\beta} & =e_{\beta}^{\top} \int_{\mathbb{R}^{d}} \Xi^{\beta}\left(x, \alpha^{*}, \boldsymbol{\beta}_{0}\right) \mathrm{d} \mu_{\left(\alpha^{*}, \boldsymbol{\beta}_{0}\right)}(x) e_{\beta}=\frac{1}{\left(\alpha^{*}\right)^{2}}(0,1)\left(\begin{array}{cc}
\frac{\left(\alpha^{*}\right)^{2}}{2 \beta^{*}} & 0 \\
0 & \left(\beta^{*}\right)^{2}
\end{array}\right)\left(\begin{array}{l}
0 \\
1
\end{array}\right)=\left(\frac{\beta^{*}}{\alpha^{*}}\right)^{2}, \\
\mathbb{G}(v) & =-2 \mathcal{J}_{\beta}^{1 / 2} \mathcal{W}(v)+\mathcal{J}_{\beta}|v| \stackrel{d}{=}-2\left(\mathcal{W}\left(\mathcal{J}_{\beta} v\right)-\frac{1}{2}\left|\mathcal{J}_{\beta} v\right|\right) .
\end{aligned}
$$

By Theorem 3, we have that

$$
T \vartheta_{\beta}^{2}\left(\hat{\tau}_{n}^{\beta}-\tau_{*}^{\beta}\right) \stackrel{d}{\longrightarrow} \underset{v \in \mathbb{R}}{\operatorname{argmin}} \mathbb{G}(v) .
$$

Since Figure 4 shows that the distribution of the estimator is similar to the asymptotic distribution in Theorem 3 , the estimator has good behavior.

6.2. Case B. In this subsection, we consider the hyperbolic diffusion model

$$
\mathrm{d} X_{t}=\left(\beta-\frac{\gamma X_{t}}{\sqrt{1+X_{t}^{2}}}\right) \mathrm{d} t+\alpha \mathrm{d} W_{t}, \quad X_{0}=x_{0} . \quad(\alpha>0, \beta \in \mathbb{R}, \gamma>|\beta|) .
$$


TABLE 2. Mean and standard deviation of the estimators under $n=10^{6}, T \approx 3.73 \times 10^{2}$, $h \approx 3.73 \times 10^{-4}, \tau_{*}^{\beta}=0.5, \beta^{*}=2.5, \gamma_{1}^{*} \approx 5.1778, \gamma_{2}^{*}=5$ in Case $\mathrm{A}$.

\begin{tabular}{ccccc}
\hline$\hat{\beta}_{1}$ & $\hat{\gamma}_{1}$ & $\hat{\beta}_{2}$ & $\hat{\gamma}_{2}$ & $\hat{\tau}_{n}^{\beta}$ \\
\hline 2.54981 & 5.17730 & 2.54314 & 4.99981 & 0.49797 \\
$(0.22565)$ & $(0.02047)$ & $(0.24675)$ & $(0.02030)$ & $(0.01341)$ \\
\hline
\end{tabular}
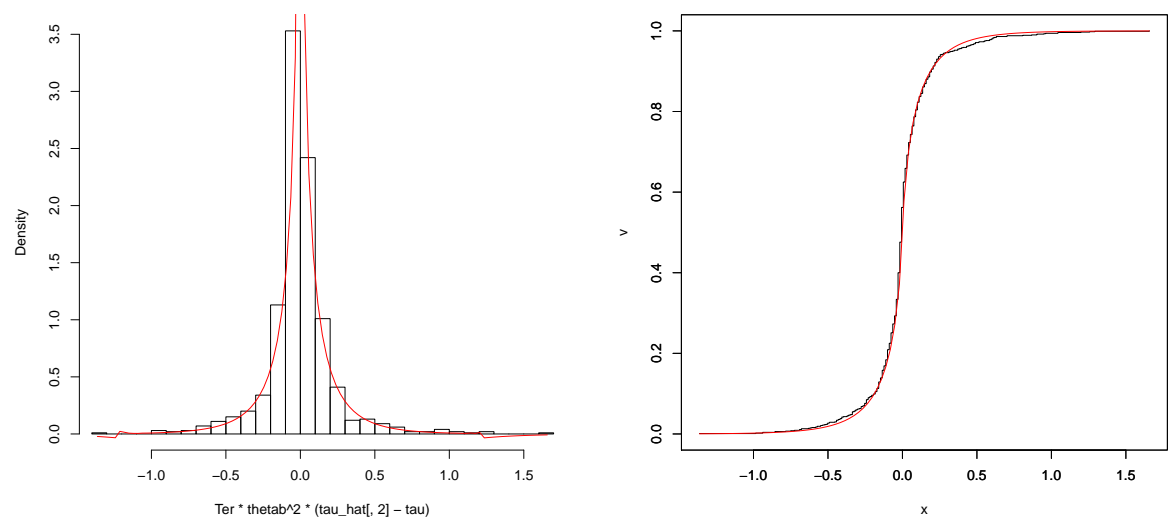

Figure 4. The figure on the left is the histogram of $T \vartheta_{\beta}^{2}\left(\hat{\tau}_{n}^{\beta}-\tau_{*}^{\beta}\right)$ (black line) and the theoretical density function (red line). The figure on the right is the empirical distribution function of $T \vartheta_{\beta}^{2}\left(\hat{\tau}_{n}^{\beta}-\tau_{*}^{\beta}\right)$ (black line) and the theoretical distribution function (red line).

For simulations of the estimator $\hat{\tau}_{n}^{\alpha}$ in Case B, we study the following stochastic differential equation

$$
X_{t}=\left\{\begin{array}{l}
X_{0}+\int_{0}^{t}\left(\beta^{*}-\frac{\gamma^{*} X_{s}}{\sqrt{1+X_{s}^{2}}}\right) \mathrm{d} s+\alpha_{1}^{*} W_{t}, \quad t \in\left[0, \tau_{*}^{\beta} T\right), \\
X_{\tau_{*}^{\beta} T}+\int_{\tau_{*}^{\beta} T}^{t}\left(\beta^{*}-\frac{\gamma^{*} X_{s}}{\sqrt{1+X_{s}^{2}}}\right) \mathrm{d} s+\alpha_{2}^{*}\left(W_{t}-W_{\tau_{*}^{\beta} T}\right), \quad t \in\left[\tau_{*}^{\beta} T, T\right],
\end{array}\right.
$$

where $x_{0}=2, \beta^{*}=0, \gamma^{*}=1, \tau_{*}^{\alpha}=0.5, \alpha_{1}^{*}=1, \alpha_{2}^{*}=2$. The number of iteration is 1000 . We set that the sample size of the data $\left\{X_{t_{i}}\right\}_{i=0}^{n}$ is $n=10^{5}$ or $10^{6}$ and $h_{n}=n^{-2 / 3}$. The existence of a change point in the intervals $[1 / 4 T, T]$ and $[0,3 / 4 T]$ was investigated and the change point was detected. Therefore, we estimated $\hat{\alpha}_{1}$ and $\hat{\alpha}_{2}$ with $\underline{\tau}^{\alpha}=1 / 4$ and $\bar{\tau}^{\alpha}=3 / 4$, respectively. The estimates of $\alpha_{1}^{*}, \alpha_{2}^{*}$ and $\tau_{*}^{\alpha}$ are reported in Table 3 . By Theorem 2, one has that

$$
n\left(\hat{\tau}_{n}^{\alpha}-\tau_{*}^{\alpha}\right)=O_{p}(1) .
$$

Since Figure 5 shows that $n\left(\hat{\tau}_{n}^{\alpha}-\tau_{*}^{\alpha}\right)$ does not diverges when increasing from $n=10^{5}$ to $n=10^{6}$, it seems that $n\left(\hat{\tau}_{n}^{\alpha}-\tau_{*}^{\alpha}\right)$ is $O_{p}(1)$ in this example.

TABLE 3. Mean and standard deviation of the estimators under $\tau_{*}^{\alpha}=0.5, \alpha_{1}^{*}=1, \alpha_{2}^{*}=2$ in Case B.

\begin{tabular}{cccccc}
\hline$n$ & $T$ & $h$ & $\hat{\alpha}_{1}$ & $\hat{\alpha}_{2}$ & $\hat{\tau}_{n}^{\alpha}$ \\
\hline $10^{5}$ & 46.4 & $4.64 \times 10^{-4}$ & 0.99976 & 2.00044 & 0.49521 \\
& & & $(0.00471)$ & $(0.00927)$ & $(0.00018)$ \\
$10^{6}$ & $10^{2}$ & $10^{-4}$ & 1.00003 & 1.99998 & 0.49966 \\
& & & $(0.00143)$ & $(0.00287)$ & $(0.00012)$ \\
\hline
\end{tabular}



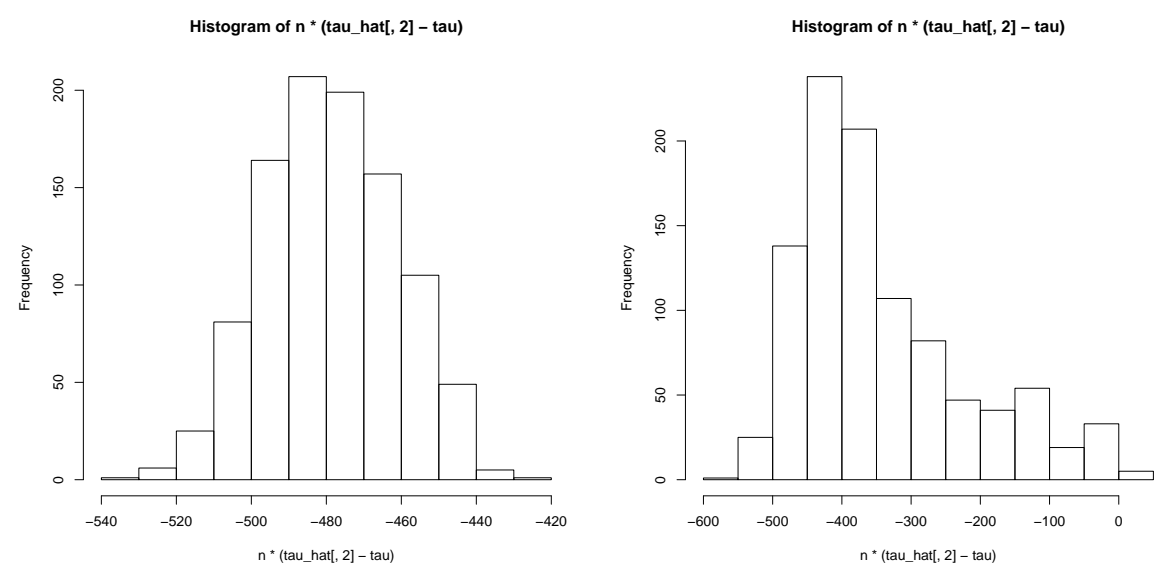

FiguRE 5 . The figure on the left is the histogram of $n\left(\hat{\tau}_{n}^{\alpha}-\tau_{*}^{\alpha}\right)$ with $n=10^{5}$. The figure on the right is the histogram of $n\left(\hat{\tau}_{n}^{\alpha}-\tau_{*}^{\alpha}\right)$ with $n=10^{6}$.

In order to investigate the asymptotic performance of the estimator $\hat{\tau}_{n}^{\beta}$ in Case $\mathrm{B}$, we consider the stochastic differential equation as follows.

$$
X_{t}=\left\{\begin{array}{l}
X_{0}+\int_{0}^{t}\left(\beta_{1}^{*}-\frac{\gamma^{*} X_{s}}{\sqrt{1+X_{s}^{2}}}\right) \mathrm{d} s+\alpha^{*} W_{t}, \quad t \in\left[0, \tau_{*}^{\beta} T\right), \\
X_{\tau_{*}^{\beta} T}+\int_{\tau_{*}^{\beta} T}^{t}\left(\beta_{2}^{*}-\frac{\gamma^{*} X_{s}}{\sqrt{1+X_{s}^{2}}}\right) \mathrm{d} s+\alpha^{*}\left(W_{t}-W_{\tau_{*}^{\beta} T}\right), \quad t \in\left[\tau_{*}^{\beta} T, T\right],
\end{array}\right.
$$

where $x_{0}=0.25, \alpha^{*}=0.2, \gamma^{*}=1.2, \tau_{*}^{\beta}=0.5, \beta_{1}^{*}=0.25, \beta_{2}^{*}=-0.25$. The number of iteration is 1000 . We set that the sample size of the data $\left\{X_{t_{i}}\right\}_{i=0}^{n}$ is $n=10^{6}$ or $10^{7}$ and $h_{n}=n^{-4 / 7}$. In all iterations, the change point was detected in the intervals $[1 / 4 T, T]$ and $[0,3 / 4 T]$. Therefore, we estimated $\hat{\beta}_{1}$ and $\hat{\beta}_{2}$ with $\underline{\tau}^{\beta}=1 / 4$ and $\bar{\tau}^{\beta}=3 / 4$, respectively. The estimates of $\boldsymbol{\beta}_{1}^{*}, \boldsymbol{\beta}_{2}^{*}$ and $\tau_{*}^{\beta}$ are reported in Table 4 It follows from Theorem 4 that

$$
T\left(\hat{\tau}_{n}^{\beta}-\tau_{*}^{\beta}\right)=O_{p}(1) .
$$

From Figure 6. $T\left(\hat{\tau}_{n}^{\beta}-\tau_{*}^{\beta}\right)$ does not diverge when increasing from $n=10^{6}$ to $n=10^{7}$. In this example, it appears that $T\left(\hat{\tau}_{n}^{\beta}-\tau_{*}^{\beta}\right)$ satisfies $O_{p}(1)$.

TABLE 4. Mean and standard deviation of the estimators under $\tau_{*}^{\beta}=0.5, \beta_{1}^{*}=0.25$,

$\beta_{2}^{*}=-0.25, \gamma^{*}=1.2$ in Case B.

\begin{tabular}{cccccccc}
\hline$n$ & $T$ & $h$ & $\hat{\beta}_{1}$ & $\hat{\gamma}_{1}$ & $\hat{\beta}_{2}$ & $\hat{\gamma}_{2}$ & $\hat{\tau}_{n}^{\beta}$ \\
\hline $10^{6}$ & $3.73 \times 10^{2}$ & $3.73 \times 10^{-4}$ & 0.25964 & 1.24852 & -0.26001 & 1.24677 & 0.49884 \\
& & & $(0.04142)$ & $(0.16744)$ & $(0.04451)$ & $(0.18435)$ & $(0.00191)$ \\
$10^{7}$ & $10^{3}$ & $10^{-4}$ & 0.25216 & 1.21212 & -0.25489 & 1.22162 & 0.50002 \\
& & & $(0.02565)$ & $(0.10281)$ & $(0.02450)$ & $(0.09966)$ & $(0.00055)$ \\
\hline
\end{tabular}

\section{Proofs}

Let $\mathscr{G}_{i-1}^{n}=\sigma\left[\left\{W_{s}\right\}_{s \leq t_{i}^{n}}\right]$, and $C, C^{\prime}>0$ denote universal constants. If $f$ is a function on $\mathbb{R}^{d} \times \Theta, f_{i-1}(\theta)$ denotes the value $f\left(X_{t_{i-1}}, \theta\right)$. If $\left\{u_{n}\right\}$ is a positive sequence, $R$ denotes a function on $\mathbb{R}^{d} \times \mathbb{R}_{+} \times \Theta$ for which there exists a constant $C>0$ such that

$$
\sup _{\theta \in \Theta}\left\|R\left(x, u_{n}, \theta\right)\right\| \leq u_{n} C(1+\|x\|)^{C} .
$$



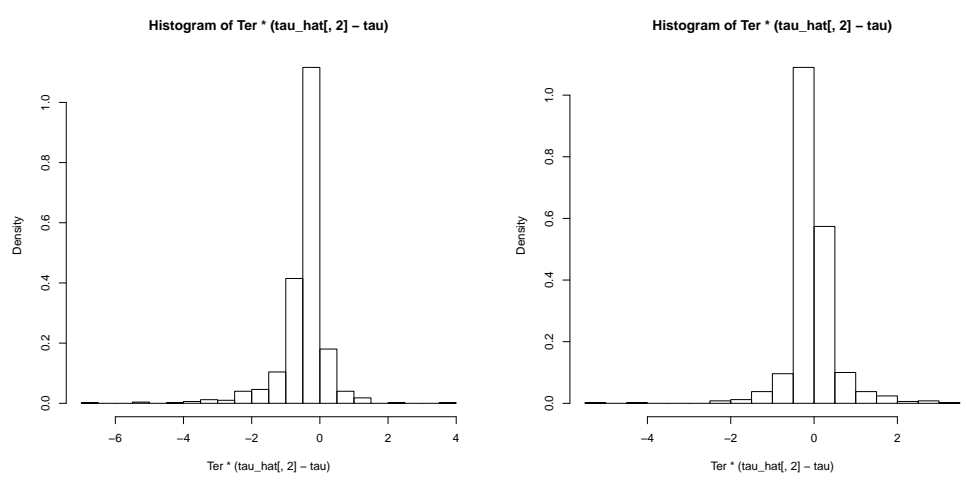

Figure 6 . The figure on the left is the histogram of $T\left(\hat{\tau}_{n}^{\beta}-\tau_{*}^{\beta}\right)$ with $n=10^{6}$. The figure on the right is the histogram of $T\left(\hat{\tau}_{n}^{\beta}-\tau_{*}^{\beta}\right)$ with $n=10^{7}$.

Let $R_{i-1}\left(u_{n}, \theta\right)=R\left(X_{t_{i-1}}, u_{n}, \theta\right)$. We set

$$
A \otimes x^{\otimes k}=\sum_{\ell_{1}, \ldots, \ell_{k}=1}^{d_{1}} A^{\ell_{1}, \ldots, \ell_{k}} x^{\ell_{1}} \cdots x^{\ell_{k}}, \quad \text { for } A \in \underbrace{\mathbb{R}^{d_{1}} \otimes \cdots \otimes \mathbb{R}^{d_{1}}}_{k}, x \in \mathbb{R}^{d_{1}} .
$$

Lemma 1 Let $\Upsilon_{n}\left(\tau: \theta_{1}, \theta_{2}\right)$ be a contrast function, and let $\hat{\theta}_{1}, \hat{\theta}_{2}$ be estimators of $\theta_{1}, \theta_{2}$, respectively, and let $\hat{\tau}_{n}=\operatorname{argmin}_{\tau \in[0,1]} \Upsilon_{n}\left(\tau: \hat{\theta}_{1}, \hat{\theta}_{2}\right)$ be the estimator of $\tau^{*}$, and let $\hat{\mathbb{H}}_{n}(v)=\Upsilon_{n}\left(\tau^{*}+v / r_{n}: \hat{\theta}_{1}, \hat{\theta}_{2}\right)-\Upsilon_{n}\left(\tau^{*}: \hat{\theta}_{1}, \hat{\theta}_{2}\right)$. If there exist a positive sequence $\left\{r_{n}\right\}$ with $r_{n} \longrightarrow \infty$ and a random field $\mathbb{H}(v)$ that satisfy the following conditions, then $r_{n}\left(\hat{\tau}_{n}-\tau^{*}\right) \stackrel{d}{\longrightarrow} \operatorname{argmin}_{v \in \mathbb{R}} \mathbb{H}(v)$.

(a) $r_{n}\left(\hat{\tau}_{n}-\tau^{*}\right)=O_{p}(1)$

(b) For all $L>0$,

$$
\hat{\mathbb{H}}_{n}(v) \stackrel{w}{\longrightarrow} \mathbb{H}(v) \quad \text { in } \mathbb{D}[-L, L] .
$$

Proof. Set $v^{\dagger}=\operatorname{argmin}_{v \in \mathbb{R}} \mathbb{H}(v)$. For all $x \in \mathbb{R}$,

$$
\begin{aligned}
P\left(r_{n}\left(\hat{\tau}_{n}-\tau^{*}\right) \leq x\right) \leq & P\left(r_{n}\left(\hat{\tau}_{n}-\tau^{*}\right) \leq x, r_{n}\left(\hat{\tau}_{n}-\tau^{*}\right) \in[-L, L], \inf _{v \in[-L, x]} \hat{\mathbb{H}}_{n}(v)>\inf _{v \in[x, L]} \hat{\mathbb{H}}_{n}(v)\right) \\
& +P\left(r_{n}\left(\hat{\tau}_{n}-\tau^{*}\right) \notin[-L, L]\right)+P\left(\inf _{v \in[-L, x]} \hat{\mathbb{H}}_{n}(v) \leq \inf _{v \in[x, L]} \hat{\mathbb{H}}_{n}(v)\right) .
\end{aligned}
$$

If $r_{n}\left(\hat{\tau}_{n}-\tau^{*}\right) \in[-L, L]$ and $\inf _{v \in[-L, x]} \hat{\mathbb{H}}_{n}(v)>\inf _{v \in[x, L]} \hat{\mathbb{H}}_{n}(v)$, then

$$
\inf _{v \in[-L, x]} \Upsilon_{n}\left(\tau^{*}+\frac{v}{r_{n}}: \hat{\theta}_{1}, \hat{\theta}_{2}\right)>\inf _{v \in[x, L]} \Upsilon_{n}\left(\tau^{*}+\frac{v}{r_{n}}: \hat{\theta}_{1}, \hat{\theta}_{2}\right),
$$

hence, from $\tau^{*}+\frac{x}{r_{n}}<\hat{\tau}_{n} \leq \tau^{*}+\frac{L}{r_{n}}, x<r_{n}\left(\hat{\tau}_{n}-\tau^{*}\right) \leq L$ and

$$
\begin{aligned}
& P\left(r_{n}\left(\hat{\tau}_{n}-\tau^{*}\right) \leq x, r_{n}\left(\hat{\tau}_{n}-\tau^{*}\right) \in[-L, L], \inf _{v \in[-L, x]} \hat{\mathbb{H}}_{n}(v)>\inf _{v \in[x, L]} \hat{\mathbb{H}}_{n}(v)\right) \\
& \leq P\left(r_{n}\left(\hat{\tau}_{n}-\tau^{*}\right) \leq x, x<r_{n}\left(\hat{\tau}_{n}-\tau^{*}\right) \leq L\right) \\
& =0 .
\end{aligned}
$$

From (b), as $n \rightarrow \infty, 7.1$ is evaluated as follows.

$$
\begin{aligned}
\varlimsup_{n \rightarrow \infty} P\left(r_{n}\left(\hat{\tau}_{n}-\tau^{*}\right) \leq x\right) & \leq \varlimsup_{n \rightarrow \infty} P\left(r_{n}\left(\hat{\tau}_{n}-\tau^{*}\right) \notin[-L, L]\right)+\varlimsup_{n \rightarrow \infty} P\left(\inf _{v \in[-L, x]} \hat{\mathbb{H}}_{n}(v) \leq \inf _{v \in[x, L]} \hat{\mathbb{H}}_{n}(v)\right) \\
& \leq \sup _{n \in \mathbb{N}} P\left(r_{n}\left(\hat{\tau}_{n}-\tau^{*}\right) \notin[-L, L]\right)+P\left(\inf _{v \in[-L, x]} \mathbb{H}(v) \leq \inf _{v \in[x, L]} \mathbb{H}(v)\right) .
\end{aligned}
$$


Now we have

$$
\begin{aligned}
P\left(\inf _{v \in[-L, x]} \mathbb{H}(v) \leq \inf _{v \in[x, L]} \mathbb{H}(v)\right) \leq & P\left(\inf _{v \in[-L, x]} \mathbb{H}(v) \leq \inf _{v \in[x, L]} \mathbb{H}(v), v^{\dagger} \in[-L, L], v^{\dagger}>x\right) \\
& +P\left(v^{\dagger} \notin[-L, L]\right)+P\left(v^{\dagger} \leq x\right) .
\end{aligned}
$$

Because if $\inf _{v \in[-L, x]} \mathbb{H}(v) \leq \inf _{v \in[x, L]} \mathbb{H}(v)$ and $v^{\dagger} \in[-L, L]$, then $-L \leq v^{\dagger} \leq x$, we have

$$
P\left(\inf _{v \in[-L, x]} \mathbb{H}(v) \leq \inf _{v \in[x, L]} \mathbb{H}(v), v^{\dagger} \in[-L, L], v^{\dagger}>x\right) \leq P\left(-L \leq v^{\dagger} \leq x, v^{\dagger}>x\right)=0 .
$$

Therefore from

$$
P\left(\inf _{v \in[-L, x]} \mathbb{H}(v) \leq \inf _{v \in[x, L]} \mathbb{H}(v)\right) \leq P\left(v^{\dagger} \notin[-L, L]\right)+P\left(v^{\dagger} \leq x\right)
$$

and (a), as $L \rightarrow \infty, 7.2$ is evaluated as follows.

$$
\begin{aligned}
\varlimsup_{n \rightarrow \infty} P\left(r_{n}\left(\hat{\tau}_{n}-\tau^{*}\right) \leq x\right) & \leq \varlimsup_{L \rightarrow \infty} \sup _{n \in \mathbb{N}} P\left(r_{n}\left(\hat{\tau}_{n}-\tau^{*}\right) \notin[-L, L]\right)+\varlimsup_{L \rightarrow \infty} P\left(v^{\dagger} \notin[-L, L]\right)+P\left(v^{\dagger} \leq x\right) \\
& =P\left(v^{\dagger} \leq x\right) .
\end{aligned}
$$

In the same way, we have

$$
\begin{aligned}
\varlimsup_{n \rightarrow \infty} P\left(r_{n}\left(\hat{\tau}_{n}-\tau^{*}\right)>x\right) & \leq \varlimsup_{n \rightarrow \infty} P\left(r_{n}\left(\hat{\tau}_{n}-\tau^{*}\right) \notin[-L, L]\right)+\varlimsup_{n \rightarrow \infty} P\left(\inf _{v \in[-L, x]} \hat{\mathbb{H}}_{n}(v) \geq \inf _{v \in[x, L]} \hat{\mathbb{H}}_{n}(v)\right) \\
& \leq \sup _{n \in \mathbb{N}} P\left(r_{n}\left(\hat{\tau}_{n}-\tau^{*}\right) \notin[-L, L]\right)+P\left(\inf _{v \in[-L, x]} \mathbb{H}(v) \geq \inf _{v \in[x, L]} \mathbb{H}(v)\right) \\
& \leq \sup _{n \in \mathbb{N}} P\left(r_{n}\left(\hat{\tau}_{n}-\tau^{*}\right) \notin[-L, L]\right)+P\left(v^{\dagger}>x\right)+P\left(v^{\dagger} \notin[-L, L]\right) .
\end{aligned}
$$

As $L \rightarrow \infty$,

$$
\varlimsup_{n \rightarrow \infty} P\left(r_{n}\left(\hat{\tau}_{n}-\tau^{*}\right)>x\right) \leq P\left(v^{\dagger}>x\right)
$$

i.e.,

$$
\begin{aligned}
\varliminf_{n \rightarrow \infty} P\left(r_{n}\left(\hat{\tau}_{n}-\tau^{*}\right) \leq x\right) & =\varliminf_{n \rightarrow \infty}\left[1-P\left(r_{n}\left(\hat{\tau}_{n}-\tau^{*}\right)>x\right)\right] \\
& =1-\varlimsup_{n \rightarrow \infty} P\left(r_{n}\left(\hat{\tau}_{n}-\tau^{*}\right)>x\right) \\
& \geq 1-P\left(v^{\dagger}>x\right) \\
& =P\left(v^{\dagger} \leq x\right) .
\end{aligned}
$$

From 7.3 and 7.4 , we obtain $\lim _{n \rightarrow \infty} P\left(r_{n}\left(\hat{\tau}_{n}-\tau^{*}\right) \leq x\right)=P\left(v^{\dagger} \leq x\right)$ and $r_{n}\left(\hat{\tau}_{n}-\tau^{*}\right) \stackrel{d}{\longrightarrow} v^{\dagger}$.

In Case A of Situation I, we set

$$
\begin{aligned}
& \mathbb{F}_{n}(v)=\Phi_{n}\left(\tau_{*}^{\alpha}+\frac{v}{n \vartheta_{\alpha}^{2}}: \alpha_{1}^{*}, \alpha_{2}^{*}\right)-\Phi_{n}\left(\tau_{*}^{\alpha}: \alpha_{1}^{*}, \alpha_{2}^{*}\right), \\
& \hat{\mathbb{F}}_{n}(v)=\Phi_{n}\left(\tau_{*}^{\alpha}+\frac{v}{n \vartheta_{\alpha}^{2}}: \hat{\alpha}_{1}, \hat{\alpha}_{2}\right)-\Phi_{n}\left(\tau_{*}^{\alpha}: \hat{\alpha}_{1}, \hat{\alpha}_{2}\right), \\
& \mathcal{D}_{n}^{\alpha}(v)=\hat{\mathbb{F}}_{n}(v)-\mathbb{F}_{n}(v) .
\end{aligned}
$$

Lemma 2 Suppose that $[\mathbf{C 1}]-[\mathbf{C 5}],[\mathbf{C 6}-\mathbf{I}],[\mathbf{A 1 - I}]$ and $[\mathbf{A 2 - I}]$ hold. Then, for all $L>0$,

$$
\sup _{v \in[-L, L]}\left|\mathcal{D}_{n}^{\alpha}(v)\right| \stackrel{p}{\longrightarrow} 0
$$

as $n \rightarrow \infty$. 
Proof. We assume that $v>0$. Then, we can express

$$
\begin{aligned}
\mathcal{D}_{n}^{\alpha}(v)= & \sum_{i=\left[n \tau_{*}^{\alpha}\right]+1}^{\left[n \tau_{*}^{\alpha}+v / \vartheta_{\alpha}^{2}\right]}\left(F_{i}\left(\hat{\alpha}_{1}\right)-F_{i}\left(\hat{\alpha}_{2}\right)\right)-\sum_{i=\left[n \tau_{*}^{\alpha}\right]+1}^{\left[n \tau_{*}^{\alpha}+v / \vartheta_{\alpha}^{2}\right]}\left(F_{i}\left(\alpha_{1}^{*}\right)-F_{i}\left(\alpha_{2}^{*}\right)\right) \\
= & \sum_{i=\left[n \tau_{*}^{\alpha}\right]+1}^{\left[n \tau_{*}^{\alpha}+v / \vartheta_{\alpha}^{2}\right]}\left[\left(F_{i}\left(\alpha_{1}^{*}\right)+\partial_{\alpha} F_{i}\left(\alpha_{1}^{*}\right)\left(\hat{\alpha}_{1}-\alpha_{1}^{*}\right)+\int_{0}^{1} \partial_{\alpha}^{2} F_{i}\left(\alpha_{1}^{*}+u\left(\hat{\alpha}_{1}-\alpha_{1}^{*}\right)\right) \mathrm{d} u \otimes\left(\hat{\alpha}_{1}-\alpha_{1}^{*}\right)^{\otimes 2}\right)\right. \\
& \left.-\left(F_{i}\left(\alpha_{2}^{*}\right)+\partial_{\alpha} F_{i}\left(\alpha_{2}^{*}\right)\left(\hat{\alpha}_{2}-\alpha_{2}^{*}\right)+\int_{0}^{1} \partial_{\alpha}^{2} F_{i}\left(\alpha_{2}^{*}+u\left(\hat{\alpha}_{2}-\alpha_{2}^{*}\right)\right) \mathrm{d} u \otimes\left(\hat{\alpha}_{2}-\alpha_{2}^{*}\right)^{\otimes 2}\right)\right] \\
= & \sum_{i=\left[n \tau_{*}^{\alpha}\right]+1}^{\left[n \tau_{*}^{\alpha}+v / \vartheta_{\alpha}^{2}\right]}\left(\partial_{\alpha} F_{i}\left(\alpha_{1}^{*}\right)\left(\hat{\alpha}_{1}-\alpha_{1}^{*}\right)+\partial_{\alpha} F_{i}\left(\alpha_{2}^{*}\right)\left(\hat{\alpha}_{2}-\alpha_{2}^{*}\right)\right) \\
& \left.+\sum_{\left.i n \tau_{*}^{\alpha}+v / \vartheta_{\alpha}^{2}\right]} \sum_{\left.i n \tau_{*}^{\alpha}\right]+1}^{\left[n \vartheta_{\alpha}^{2}\right]}\left(\int_{0}^{1} \partial_{\alpha}^{2} F_{i}\left(\alpha_{1}^{*}+u\left(\hat{\alpha}_{1}^{*}\right)-\alpha_{1}^{*}\right)\right) \mathrm{d} u \otimes\left(\hat{\alpha}_{1}-\alpha_{1}^{*}\right)\right) \\
& \left.-\int_{0}^{1} \partial_{\alpha}^{2} F_{i}\left(\alpha_{2}^{*}+u\left(\hat{\alpha}_{2}-\alpha_{2}^{*}\right)\right) \mathrm{d} u \otimes\left(\hat{\alpha}_{2}-\alpha_{2}^{*}\right)^{\otimes 2}\right) .
\end{aligned}
$$

Now we see

$$
\begin{aligned}
& \sup _{v \in[0, L]}\left|\sum_{i=\left[n \tau_{*}^{\alpha}\right]+1}^{\left[n \tau_{*}^{\alpha}+v / \vartheta_{\alpha}^{2}\right]} \int_{0}^{1} \partial_{\alpha}^{2} F_{i}\left(\alpha_{k}^{*}+u\left(\hat{\alpha}_{k}-\alpha_{k}^{*}\right)\right) \mathrm{d} u \otimes\left(\hat{\alpha}_{k}-\alpha_{k}^{*}\right)^{\otimes 2}\right| \\
& \leq \sum_{i=\left[n \tau_{*}^{\alpha}\right]+1}^{\left[n \tau_{*}^{\alpha}+L / \vartheta_{\alpha}^{2}\right]}\left|\int_{0}^{1} \partial_{\alpha}^{2} F_{i}\left(\alpha_{k}^{*}+u\left(\hat{\alpha}_{k}-\alpha_{k}^{*}\right)\right) \mathrm{d} u\right|\left|\hat{\alpha}_{k}-\alpha_{k}^{*}\right|^{2} \\
& \leq \frac{1}{n} \sum_{i=\left[n \tau_{*}^{\alpha}\right]+1}^{\left[n \tau_{*}^{\alpha}+L / \vartheta_{\alpha}^{2}\right]} \sup _{\alpha \in \Theta_{A}}\left|\partial_{\alpha}^{2} F_{i}(\alpha)\right|\left(\sqrt{n}\left|\hat{\alpha}_{k}-\alpha_{k}^{*}\right|\right)^{2} \\
& =O_{p}\left(\frac{1}{n \vartheta_{\alpha}^{2}}\right)=o_{p}(1)
\end{aligned}
$$

and

$$
\begin{aligned}
& \sum_{i=\left[n \tau_{*}^{\alpha}\right]+1}^{\left[n \tau_{*}^{\alpha}+v / \vartheta_{\alpha}^{2}\right]} \partial_{\alpha} F_{i}\left(\alpha_{k}^{*}\right)\left(\hat{\alpha}_{k}-\alpha_{k}^{*}\right) \\
= & \sum_{i=\left[n \tau_{*}^{\alpha}\right]+1}^{\left[n \tau_{*}^{\alpha}+v / \vartheta_{\alpha}^{2}\right]}\left(\partial_{\alpha} F_{i}\left(\alpha_{k}^{*}\right)-\mathbb{E}_{\alpha_{2}^{*}}\left[\partial_{\alpha} F_{i}\left(\alpha_{k}^{*}\right) \mid \mathscr{G}_{i-1}^{n}\right]\right)\left(\hat{\alpha}_{k}-\alpha_{k}^{*}\right)+\sum_{i=\left[n \tau_{*}^{\alpha}\right]+1}^{\left[n \tau_{*}^{\alpha}+v / \vartheta_{\alpha}^{2}\right]} \mathbb{E}_{\alpha_{2}^{*}}\left[\partial_{\alpha} F_{i}\left(\alpha_{k}^{*}\right) \mid \mathscr{G}_{i-1}^{n}\right]\left(\hat{\alpha}_{k}-\alpha_{k}^{*}\right) .
\end{aligned}
$$

By Theorem 2.11 of Hall and Heyde (1980), we have

$$
\begin{aligned}
& \mathbb{E}_{\alpha_{2}^{*}}\left[\frac{1}{n} \sup _{v \in[0, L]}\left|\sum_{i=\left[n \tau_{*}^{\alpha}\right]+1}^{\left[n \tau_{*}^{\alpha}+v / \vartheta_{\alpha}^{2}\right]}\left(\partial_{\alpha} F_{i}\left(\alpha_{k}^{*}\right)-\mathbb{E}_{\alpha_{2}^{*}}\left[\partial_{\alpha} F_{i}\left(\alpha_{k}^{*}\right) \mid \mathscr{G}_{i-1}^{n}\right]\right)\right|^{2}\right] \\
& \leq \frac{C}{n} \sum_{i=\left[n \tau_{*}^{\alpha}\right]+1}^{\left[n \tau_{*}^{\alpha}+L / \vartheta_{\alpha}^{2}\right]} \mathbb{E}_{\alpha_{2}^{*}}\left[\mathbb{E}_{\alpha_{2}^{*}}\left[\left|\partial_{\alpha} F_{i}\left(\alpha_{k}^{*}\right)-\mathbb{E}_{\alpha_{2}^{*}}\left[\partial_{\alpha} F_{i}\left(\alpha_{k}^{*}\right) \mid \mathscr{G}_{i-1}^{n}\right]\right|^{2} \mid \mathscr{G}_{i-1}^{n}\right]\right]
\end{aligned}
$$




$$
\leq \frac{C^{\prime}}{n \vartheta_{\alpha}^{2}} \longrightarrow 0
$$

and

$$
\frac{1}{\sqrt{n}} \sup _{v \in[0, L]}\left|\sum_{i=\left[n \tau_{*}^{\alpha}\right]+1}^{\left[n \tau_{*}^{\alpha}+v / \vartheta_{\alpha}^{2}\right]}\left(\partial_{\alpha} F_{i}\left(\alpha_{k}^{*}\right)-\mathbb{E}_{\alpha_{2}^{*}}\left[\partial_{\alpha} F_{i}\left(\alpha_{k}^{*}\right) \mid \mathscr{G}_{i-1}^{n}\right]\right)\right|=o_{p}(1) .
$$

Moreover, we see

$$
\begin{aligned}
& \sup _{v \in[0, L]}\left|\sum_{i=\left[n \tau_{*}^{\alpha}\right]+1}^{\left[n \tau_{*}^{\alpha}+v / \vartheta_{\alpha}^{2}\right]} \mathbb{E}_{\alpha_{2}^{*}}\left[\partial_{\alpha} F_{i}\left(\alpha_{k}^{*}\right) \mid \mathscr{G}_{i-1}^{n}\right]\right|\left|\hat{\alpha}_{k}-\alpha_{k}^{*}\right| \\
& =\sup _{v \in[0, L]}\left|\sum_{i=\left[n \tau_{*}^{\alpha}\right]+1}^{\left[n \tau_{*}^{\alpha}+v / \vartheta_{\alpha}^{2}\right]}\left(\left[\operatorname{tr}\left(A_{i-1}^{-1} \partial_{\alpha^{\ell}} A_{i-1} A_{i-1}^{-1}\left(\alpha_{k}^{*}\right)\left(A_{i-1}\left(\alpha_{2}^{*}\right)-A_{i-1}\left(\alpha_{k}^{*}\right)\right)\right)\right]_{\ell}+R_{i-1}(h, \theta)\right)\right|\left|\hat{\alpha}_{k}-\alpha_{k}^{*}\right| \\
& =\sup _{v \in[0, L]} \mid \sum_{i=\left[n \tau_{*}^{\alpha}\right]+1}^{\left[n \tau_{*}^{\alpha}+v / \vartheta_{\alpha}^{2}\right]}\left(\Xi_{i-1}^{\alpha}\left(\alpha_{k}^{*}\right)\left(\alpha_{2}^{*}-\alpha_{k}^{*}\right)\right. \\
& +\int_{0}^{1}(1-u)\left(\operatorname{tr}\left[A_{i-1}^{-1} \partial_{\alpha^{\ell_{1}}} A_{i-1} A_{i-1}^{-1}\left(\alpha_{k}^{*}\right) \partial_{\alpha^{\ell_{3}}} \partial_{\alpha^{\ell_{2}}} A_{i-1}\left(\alpha_{k}^{*}+u\left(\alpha_{2}^{*}-\alpha_{k}^{*}\right)\right)\right]\right)_{\ell_{1}, \ell_{2}, \ell_{3}} \mathrm{~d} u \otimes\left(\alpha_{2}^{*}-\alpha_{k}^{*}\right)^{\otimes 2} \\
& \left.+R_{i-1}(h, \theta)\right)|| \hat{\alpha}_{k}-\alpha_{k}^{*} \mid \\
& =\sup _{v \in[0, L]} \mid \sum_{i=\left[n \tau_{*}^{\alpha}\right]+1}^{\left[n \tau_{*}^{\alpha}+v / \vartheta_{\alpha}^{2}\right]}\left(\Xi_{i-1}^{\alpha}\left(\alpha_{0}\right)\left(\alpha_{2}^{*}-\alpha_{k}^{*}\right)+\int_{0}^{1} \partial_{\alpha} \Xi_{i-1}^{\alpha}\left(\alpha_{0}+u\left(\alpha_{k}^{*}-\alpha_{0}\right)\right) \mathrm{d} u \otimes\left(\alpha_{2}^{*}-\alpha_{k}^{*}\right) \otimes\left(\alpha_{k}^{*}-\alpha_{0}\right)\right. \\
& +\int_{0}^{1}(1-u)\left(\operatorname{tr}\left[A_{i-1}^{-1} \partial_{\alpha^{\ell_{1}}} A_{i-1} A_{i-1}^{-1}\left(\alpha_{k}^{*}\right) \partial_{\alpha^{\ell_{3}}} \partial_{\alpha^{\ell_{2}}} A_{i-1}\left(\alpha_{k}^{*}+u\left(\alpha_{2}^{*}-\alpha_{k}^{*}\right)\right)\right]\right)_{\ell_{1}, \ell_{2}, \ell_{3}} \mathrm{~d} u \otimes\left(\alpha_{2}^{*}-\alpha_{k}^{*}\right)^{\otimes 2} \\
& \left.+R_{i-1}(h, \theta)\right)|| \hat{\alpha}_{k}-\alpha_{k}^{*} \mid \\
& \leq \frac{\vartheta_{\alpha}}{\sqrt{n}} \sup _{v \in[0, L]}\left|\sum_{i=\left[n \tau_{*}^{\alpha}\right]+1}^{\left[n \tau_{*}^{\alpha}+v / \vartheta_{\alpha}^{2}\right]} \Xi_{i-1}^{\alpha}\left(\alpha_{0}\right)\right| \vartheta_{\alpha}^{-1}\left|\alpha_{2}^{*}-\alpha_{k}^{*}\right| \sqrt{n}\left|\hat{\alpha}_{k}-\alpha_{k}^{*}\right| \\
& +\frac{\vartheta_{\alpha}^{2}}{\sqrt{n}} \sum_{i=\left[n \tau_{*}^{\alpha}\right]+1}^{\left[n \tau_{*}^{\alpha}+L / \vartheta_{\alpha}^{2}\right]} R_{i-1}(1, \theta) \vartheta_{\alpha}^{-1}\left|\alpha_{2}^{*}-\alpha_{k}^{*}\right| \vartheta_{\alpha}^{-1}\left|\alpha_{2}^{*}-\alpha_{0}\right| \sqrt{n}\left|\hat{\alpha}_{k}-\alpha_{k}^{*}\right| \\
& +\frac{\vartheta_{\alpha}^{2}}{\sqrt{n}} \sum_{i=\left[n \tau_{*}^{\alpha}\right]+1}^{\left[n \tau_{*}^{\alpha}+L / \vartheta_{\alpha}^{2}\right]} R_{i-1}(1, \theta)\left(\vartheta_{\alpha}^{-1}\left|\alpha_{2}^{*}-\alpha_{k}^{*}\right|\right)^{2} \sqrt{n}\left|\hat{\alpha}_{k}-\alpha_{k}^{*}\right|+\frac{1}{\sqrt{n}} \sum_{i=\left[n \tau_{*}^{\alpha}\right]+1}^{\left[n \tau_{*}^{\alpha}+L / \vartheta_{\alpha}^{2}\right]} R_{i-1}(h, \theta) \sqrt{n}\left|\hat{\alpha}_{k}-\alpha_{k}^{*}\right| \\
& =O_{p}\left(\frac{1}{\sqrt{n} \vartheta_{\alpha}}\right)+O_{p}\left(\frac{1}{\sqrt{n}}\right)+O_{p}\left(\frac{h}{\sqrt{n} \vartheta_{\alpha}^{2}}\right) \\
& =o_{p}(1) \text {. }
\end{aligned}
$$

Therefore, from (7.5)-(7.9), we have

$$
\sup _{v \in[0, L]}\left|\mathcal{D}_{n}^{\alpha}(v)\right| \stackrel{p}{\longrightarrow} 0 .
$$

By the similar proof, we see $\sup _{v \in[-L, 0]}\left|\mathcal{D}_{n}^{\alpha}(v)\right| \stackrel{p}{\longrightarrow} 0$ and this proof is complete.

Lemma 3 Suppose that $[\mathbf{C 1}]-[\mathbf{C 5}],[\mathbf{C 6}-\mathbf{I}],[\mathbf{A 1 - I}]$ and $[\mathbf{A 2 - I}]$ hold. Then, for all $L>0$,

$$
\mathbb{F}_{n}(v) \stackrel{w}{\longrightarrow} \mathbb{F}(v) \text { in } \mathbb{D}[-L, L]
$$


as $n \rightarrow \infty$.

Proof. We consider $v>0$. We have

$$
\begin{aligned}
\mathbb{F}_{n}(v)= & \sum_{i=\left[n \tau_{*}^{\alpha}\right]+1}^{\left[n \tau_{*}^{\alpha}+v / \vartheta_{\alpha}^{2}\right]}\left(F_{i}\left(\alpha_{1}^{*}\right)-F_{i}\left(\alpha_{2}^{*}\right)\right) \\
= & \sum_{i=\left[n \tau_{*}^{\alpha}\right]+1}^{\left[n \tau_{*}^{\alpha}+v / \vartheta_{\alpha}^{2}\right]}\left(\partial_{\alpha} F_{i}\left(\alpha_{2}^{*}\right)\left(\alpha_{1}^{*}-\alpha_{2}^{*}\right)+\frac{1}{2} \partial_{\alpha}^{2} F_{i}\left(\alpha_{2}^{*}\right) \otimes\left(\alpha_{1}^{*}-\alpha_{2}^{*}\right)^{\otimes 2}\right. \\
& \left.\quad+\int_{0}^{1} \frac{(1-u)^{2}}{2} \partial_{\alpha}^{3} F_{i}\left(\alpha_{2}^{*}+u\left(\alpha_{1}^{*}-\alpha_{2}^{*}\right)\right) \mathrm{d} u \otimes\left(\alpha_{1}^{*}-\alpha_{2}^{*}\right)^{\otimes 3}\right) \\
= & \sum_{i=\left[n \tau_{*}^{\alpha}\right]+1}^{\left[n \tau_{*}^{\alpha}+v / \vartheta_{\alpha}^{2}\right]}\left(\partial_{\alpha} F_{i}\left(\alpha_{2}^{*}\right)\left(\alpha_{1}^{*}-\alpha_{2}^{*}\right)+\frac{1}{2} \partial_{\alpha}^{2} F_{i}\left(\alpha_{2}^{*}\right) \otimes\left(\alpha_{1}^{*}-\alpha_{2}^{*}\right)^{\otimes 2}\right)+\bar{o}_{p}(1) \\
= & : \mathbb{F}_{1, n}(v)+\mathbb{F}_{2, n}(v)+\bar{o}_{p}(1),
\end{aligned}
$$

where $Y_{n}(v)=\bar{o}_{p}(1) \operatorname{denotes} \sup _{v \in[0, L]}\left|Y_{n}(v)\right|=o_{p}(1)$. Now, we see

$$
\begin{aligned}
& \mathbb{F}_{1, n}(v)=\sum_{i=\left[n \tau_{*}^{\alpha}\right]+1}^{\left[n \tau_{*}^{\alpha}+v / \vartheta_{\alpha}^{2}\right]}\left(\partial_{\alpha} F_{i}\left(\alpha_{2}^{*}\right)-\mathbb{E}_{\alpha_{2}^{*}}\left[\partial_{\alpha} F_{i}\left(\alpha_{2}^{*}\right) \mid \mathscr{G}_{i-1}^{n}\right]\right)\left(\alpha_{1}^{*}-\alpha_{2}^{*}\right)+\sum_{i=\left[n \tau_{*}^{\alpha}\right]+1}^{\left[n \tau_{*}^{\alpha}+v / \vartheta_{\alpha}^{2}\right]} \mathbb{E}_{\alpha_{2}^{*}}\left[\partial_{\alpha} F_{i}\left(\alpha_{2}^{*}\right) \mid \mathscr{G}_{i-1}^{n}\right]\left(\alpha_{1}^{*}-\alpha_{2}^{*}\right), \\
& \sup _{v \in[0, L]}\left|\sum_{i=\left[n \tau_{*}^{\alpha}\right]+1}^{\left[n \tau_{*}^{\alpha}+v / \vartheta_{\alpha}^{2}\right]} \mathbb{E}_{\alpha_{2}^{*}}\left[\partial_{\alpha} F_{i}\left(\alpha_{2}^{*}\right) \mid \mathscr{G}_{i-1}^{n}\right]\left(\alpha_{1}^{*}-\alpha_{2}^{*}\right)\right| \leq \vartheta_{\alpha} \sum_{i=\left[n \tau_{*}^{\alpha}\right]+1}^{\left[n \tau_{*}^{\alpha}+L / \vartheta_{\alpha}^{2}\right]} R_{i-1}(h, \theta)=O_{p}\left(\frac{h}{\vartheta_{\alpha}}\right)=o_{p}(1), \\
& \sum_{i=\left[n \tau_{*}^{\alpha}\right]+1}^{\left[n \tau_{*}^{\alpha}+v / \vartheta_{\alpha}^{2}\right]} \mathbb{E}_{\alpha_{2}^{*}}\left[\left(\left(\partial_{\alpha} F_{i}\left(\alpha_{2}^{*}\right)-\mathbb{E}_{\alpha_{2}^{*}}\left[\partial_{\alpha} F_{i}\left(\alpha_{2}^{*}\right) \mid \mathscr{G}_{i-1}^{n}\right]\right)\left(\alpha_{1}^{*}-\alpha_{2}^{*}\right)\right)^{2} \mid \mathscr{G}_{i-1}^{n}\right] \\
& =\left(\alpha_{1}^{*}-\alpha_{2}^{*}\right)^{\top} \sum_{i=\left[n \tau_{*}^{\alpha}\right]+1}^{\left[n \tau_{*}^{\alpha}+v / \vartheta_{\alpha}^{2}\right]} \mathbb{E}_{\alpha_{2}^{*}}\left[\left(\partial_{\alpha} F_{i}\left(\alpha_{2}^{*}\right)-\mathbb{E}_{\alpha_{2}^{*}}\left[\partial_{\alpha} F_{i}\left(\alpha_{2}^{*}\right) \mid \mathscr{G}_{i-1}^{n}\right]\right)^{\top}\right. \\
& \left.\left(\partial_{\alpha} F_{i}\left(\alpha_{2}^{*}\right)-\mathbb{E}_{\alpha_{2}^{*}}\left[\partial_{\alpha} F_{i}\left(\alpha_{2}^{*}\right) \mid \mathscr{G}_{i-1}^{n}\right]\right) \mid \mathscr{G}_{i-1}^{n}\right]\left(\alpha_{1}^{*}-\alpha_{2}^{*}\right) \\
& =\left(\alpha_{1}^{*}-\alpha_{2}^{*}\right)^{\top} \sum_{i=\left[n \tau_{*}^{\alpha}\right]+1}^{\left[n \tau_{*}^{\alpha}+v / \vartheta_{\alpha}^{2}\right]}\left(2 \Xi_{i-1}^{\alpha}\left(\alpha_{2}^{*}\right)+R_{i-1}(h, \theta)\right)\left(\alpha_{1}^{*}-\alpha_{2}^{*}\right) \\
& \stackrel{p}{\longrightarrow} 2 e_{\alpha}^{\top} \int_{\mathbb{R}^{d}} \Xi^{\alpha}\left(x, \alpha_{0}\right) \mathrm{d} \mu_{\alpha_{0}}(x) e_{\alpha} v=4 \mathcal{J}_{\alpha} v
\end{aligned}
$$

and

$$
\sum_{i=\left[n \tau_{*}^{\alpha}\right]+1}^{\left[n \tau_{*}^{\alpha}+v / \vartheta_{\alpha}^{2}\right]} \mathbb{E}_{\alpha_{2}^{*}}\left[\left(\left(\partial_{\alpha} F_{i}\left(\alpha_{2}^{*}\right)-\mathbb{E}_{\alpha_{2}^{*}}\left[\partial_{\alpha} F_{i}\left(\alpha_{2}^{*}\right) \mid \mathscr{G}_{i-1}^{n}\right]\right)\left(\alpha_{1}^{*}-\alpha_{2}^{*}\right)\right)^{4} \mid \mathscr{G}_{i-1}^{n}\right]=\sum_{i=\left[n \tau_{*}^{\alpha}\right]+1}^{\left[n \tau_{*}^{\alpha}+v / \vartheta_{\alpha}^{2}\right]} \vartheta_{\alpha}^{4} R_{i-1}(1, \theta) \stackrel{p}{\longrightarrow} 0 .
$$

According to Corollary 3.8 of McLeish (1974), we obtain, from 7.12) and 7.13),

$$
\sum_{i=\left[n \tau_{*}^{\alpha}\right]+1}^{\left[n \tau_{*}^{\alpha}+v / \vartheta_{\alpha}^{2}\right]}\left(\partial_{\alpha} F_{i}\left(\alpha_{2}^{*}\right)-\mathbb{E}_{\alpha_{2}^{*}}\left[\partial_{\alpha} F_{i}\left(\alpha_{2}^{*}\right) \mid \mathscr{G}_{i-1}^{n}\right]\right)\left(\alpha_{1}^{*}-\alpha_{2}^{*}\right) \stackrel{w}{\longrightarrow}-2 \mathcal{J}_{\alpha}^{1 / 2} \mathcal{W}(v) \text { in } \mathbb{D}[0, L] .
$$

Further, from (7.10), (7.11) and (7.14), we have $\mathbb{F}_{1, n}(v) \stackrel{w}{\longrightarrow}-2 \mathcal{J}_{\alpha}^{1 / 2} \mathcal{W}(v)$ in $\mathbb{D}[0, L]$. 
Besides, we see

$$
\begin{aligned}
& \sup _{v \in[0, L]}\left|\sum_{i=\left[n \tau_{*}^{\alpha}\right]+1}^{\left[n \tau_{*}^{\alpha}+v / \vartheta_{\alpha}^{2}\right]} \partial_{\alpha}^{2} F_{i}\left(\alpha_{2}^{*}\right) \otimes\left(\alpha_{1}^{*}-\alpha_{2}^{*}\right)^{\otimes 2}-2 \mathcal{J}_{\alpha} v\right| \\
& \leq \sup _{v \in[0, L]}\left|\sum_{i=\left[n \tau_{*}^{\alpha}\right]+1}^{\left[n \tau_{*}^{\alpha}+v / \vartheta_{\alpha}^{2}\right]}\left(\partial_{\alpha}^{2} F_{i}\left(\alpha_{2}^{*}\right)-\mathbb{E}_{\alpha_{2}^{*}}\left[\partial_{\alpha}^{2} F_{i}\left(\alpha_{2}^{*}\right) \mid \mathscr{G}_{i-1}^{n}\right]\right) \otimes\left(\alpha_{1}^{*}-\alpha_{2}^{*}\right)^{\otimes 2}\right| \\
& \quad+\sup _{v \in[0, L]} \sum_{i=\left[n \tau_{*}^{\alpha}\right]+1}^{\left[n \tau^{\alpha}+v / \vartheta_{\alpha}^{2}\right]} \mathbb{E}_{\alpha_{2}^{*}}\left[\partial_{\alpha}^{2} F_{i}\left(\alpha_{2}^{*}\right) \mid \mathscr{G}_{i-1}^{n}\right] \otimes\left(\alpha_{1}^{*}-\alpha_{2}^{*}\right)^{\otimes 2}-2 \mathcal{J}_{\alpha} v \mid, \\
& \sup _{v \in[0, L]}\left|\sum_{i=\left[n \tau_{*}^{\alpha}\right]+1}^{\left[n \tau_{*}^{\alpha}+v / \vartheta_{\alpha}^{2}\right]}\left(\partial_{\alpha}^{2} F_{i}\left(\alpha_{2}^{*}\right)-\mathbb{E}_{\alpha_{2}^{*}}\left[\partial_{\alpha}^{2} F_{i}\left(\alpha_{2}^{*}\right) \mid \mathscr{G}_{i-1}^{n}\right]\right) \otimes\left(\alpha_{1}^{*}-\alpha_{2}^{*}\right)^{\otimes 2}\right| \stackrel{p}{\longrightarrow} 0
\end{aligned}
$$

and

$$
\begin{aligned}
& \sup _{v \in[0, L]}\left|\sum_{i=\left[n \tau_{*}^{\alpha}\right]+1}^{\left[n \tau_{*}^{\alpha}+v / \vartheta_{\alpha}^{2}\right]} \mathbb{E}_{\alpha_{2}^{*}}\left[\partial_{\alpha}^{2} F_{i}\left(\alpha_{2}^{*}\right) \mid \mathscr{G}_{i-1}^{n}\right] \otimes\left(\alpha_{1}^{*}-\alpha_{2}^{*}\right)^{\otimes 2}-2 \mathcal{J}_{\alpha} v\right| \\
& =\sup _{v \in[0, L]}\left|\sum_{i=\left[n \tau_{*}^{\alpha}\right]+1}^{\left[n \tau_{*}^{\alpha}+v / \vartheta_{\alpha}^{2}\right]}\left(\Xi_{i-1}^{\alpha}\left(\alpha_{2}^{*}\right)+R_{i-1}(h, \theta)\right) \otimes\left(\alpha_{1}^{*}-\alpha_{2}^{*}\right)^{\otimes 2}-2 \mathcal{J}_{\alpha} v\right| \\
& \leq \sup _{v \in[0, L]}\left|\sum_{i=\left[n \tau_{*}^{\alpha}\right]+1}^{\left[n \tau_{*}^{\alpha}+v / \vartheta_{\alpha}^{2}\right]} \Xi_{i-1}^{\alpha}\left(\alpha_{0}\right) \otimes\left(\alpha_{1}^{*}-\alpha_{2}^{*}\right)^{\otimes 2}-2 \mathcal{J}_{\alpha} v\right|+o_{p}(1) \\
& \stackrel{p}{\longrightarrow} 0,
\end{aligned}
$$

where 7.16 is obtained by

$$
\begin{aligned}
& \mathbb{E}_{\alpha_{2}^{*}}\left[\sup _{v \in[0, L]}\left|\sum_{i=\left[n \tau_{*}^{\alpha}\right]+1}^{\left[n \tau_{*}^{\alpha}+v / \vartheta_{\alpha}^{2}\right]}\left(\partial_{\alpha}^{2} F_{i}\left(\alpha_{2}^{*}\right)-\mathbb{E}_{\alpha_{2}^{*}}\left[\partial_{\alpha}^{2} F_{i}\left(\alpha_{2}^{*}\right) \mid \mathscr{G}_{i-1}^{n}\right]\right) \otimes\left(\alpha_{1}^{*}-\alpha_{2}^{*}\right)^{\otimes 2}\right|^{2}\right] \\
& \leq C \vartheta_{\alpha}^{4} \sum_{i=\left[n \tau_{*}^{\alpha}\right]+1}^{\left[n \tau_{*}^{\alpha}+L / \vartheta_{\alpha}^{2}\right]} \mathbb{E}_{\alpha_{2}^{*}}\left[\left|\partial_{\alpha}^{2} F_{i}\left(\alpha_{2}^{*}\right)-\mathbb{E}_{\alpha_{2}^{*}}\left[\partial_{\alpha}^{2} F_{i}\left(\alpha_{2}^{*}\right) \mid \mathscr{G}_{i-1}^{n}\right]\right|^{2}\right] \\
& \leq C^{\prime} \vartheta_{\alpha}^{2} \longrightarrow 0
\end{aligned}
$$

from Theorem 2.11 of Hall and Heyde (1980), and (7.17) is obtained by

$$
\begin{aligned}
& \sup _{v \in[0, L]}\left|\sum_{i=\left[n \tau_{*}^{\alpha}\right]+1}^{\left[n \tau_{*}^{\alpha}+v / \vartheta_{\alpha}^{2}\right]} \Xi_{i-1}^{\alpha}\left(\alpha_{0}\right) \otimes\left(\alpha_{1}^{*}-\alpha_{2}^{*}\right)^{\otimes 2}-2 \mathcal{J}_{\alpha} v\right| \\
& \leq \sup _{v \in\left[0, \epsilon_{n}\right]}\left|\sum_{i=\left[n \tau_{*}^{\alpha}\right]+1}^{\left[n \tau_{*}^{\alpha}+v / \vartheta_{\alpha}^{2}\right]} \Xi_{i-1}^{\alpha}\left(\alpha_{0}\right) \otimes\left(\alpha_{1}^{*}-\alpha_{2}^{*}\right)^{\otimes 2}-2 \mathcal{J}_{\alpha} v\right| \\
& +\sup _{v \in\left[\epsilon_{n}, L\right]}\left|\sum_{i=\left[n \tau_{*}^{\alpha}\right]+1}^{\left[n \tau_{*}^{\alpha}+v / \vartheta_{\alpha}^{2}\right]} \Xi_{i-1}^{\alpha}\left(\alpha_{0}\right) \otimes\left(\alpha_{1}^{*}-\alpha_{2}^{*}\right)^{\otimes 2}-2 \mathcal{J}_{\alpha} v\right| \\
& \leq \epsilon_{n} \sup _{v \in\left[0, \epsilon_{n}\right]}\left|\frac{1}{v} \sum_{i=\left[n \tau_{*}^{\alpha}\right]+1}^{\left[n \tau_{*}^{\alpha}+v / \vartheta_{\alpha}^{2}\right]} \Xi_{i-1}^{\alpha}\left(\alpha_{0}\right) \otimes\left(\alpha_{1}^{*}-\alpha_{2}^{*}\right)^{\otimes 2}-2 \mathcal{J}_{\alpha}\right|
\end{aligned}
$$




$$
\begin{aligned}
& +L \sup _{v \in\left[\epsilon_{n}, L\right]}\left|\frac{1}{v} \sum_{i=\left[n \tau_{*}^{\alpha}\right]+1}^{\left[n \tau_{*}^{\alpha}+v / \vartheta_{\alpha}^{2}\right]} \Xi_{i-1}^{\alpha}\left(\alpha_{0}\right) \otimes\left(\alpha_{1}^{*}-\alpha_{2}^{*}\right)^{\otimes 2}-2 \mathcal{J}_{\alpha}\right| \\
& \leq O_{p}\left(\epsilon_{n}\right)+L \max _{\left[n^{1 / r}\right] \leq k \leq n}\left|\frac{1}{k} \sum_{i=\left[n \tau_{*}^{\alpha}\right]+1}^{\left[n \tau_{*}^{\alpha}\right]+k} \Xi_{i-1}^{\alpha}\left(\alpha_{0}\right) \otimes\left(\vartheta_{\alpha}^{-1}\left(\alpha_{1}^{*}-\alpha_{2}^{*}\right)\right)^{\otimes 2}-2 \mathcal{J}_{\alpha}\right| \\
& =o_{p}(1) .
\end{aligned}
$$

Here $\left\{\epsilon_{n}\right\}_{n=1}^{\infty}$ is a positive sequence such that $\epsilon_{n} \longrightarrow 0$ and $\epsilon_{n} h / \vartheta_{\alpha}^{2} \longrightarrow \infty$, and $r$ is a constant with $r \in(1,2)$ and $n h^{r} \longrightarrow \infty$. From (7.15)-(7.17), we have $\sup _{v \in[0, L]}\left|\mathbb{F}_{2, n}(v)-\mathcal{J}_{\alpha} v\right| \stackrel{p}{\longrightarrow} 0$. Therefore we obtain

$$
\mathbb{F}_{n}(v) \stackrel{w}{\longrightarrow}-2 \mathcal{J}_{\alpha}^{1 / 2} \mathcal{W}(v)+\mathcal{J}_{\alpha} v \text { in } \mathbb{D}[0, L] .
$$

The argument for $v<0$ is proved as well.

Proof of Theorem 1, Let $M \geq 1$. We have

$$
P\left(n \vartheta_{\alpha}^{2}\left|\hat{\tau}_{n}^{\alpha}-\tau_{*}^{\alpha}\right|>M\right)=P\left(n \vartheta_{\alpha}^{2}\left(\hat{\tau}_{n}^{\alpha}-\tau_{*}^{\alpha}\right)>M\right)+P\left(n \vartheta_{\alpha}^{2}\left(\tau_{*}^{\alpha}-\hat{\tau}_{n}^{\alpha}\right)>M\right) .
$$

For $\tau>\tau_{*}^{\alpha}$, we have

$$
\begin{aligned}
\Phi_{n}\left(\tau: \alpha_{1}, \alpha_{2}\right)-\Phi_{n}\left(\tau_{*}^{\alpha}: \alpha_{1}, \alpha_{2}\right) & =\sum_{i=1}^{[n \tau]} F_{i}\left(\alpha_{1}\right)+\sum_{i=[n \tau]+1}^{n} F_{i}\left(\alpha_{2}\right)-\sum_{i=1}^{\left[n \tau_{*}^{\alpha}\right]} F_{i}\left(\alpha_{1}\right)-\sum_{i=\left[n \tau_{*}^{\alpha}\right]+1}^{n} F_{i}\left(\alpha_{2}\right) \\
& =\sum_{i=\left[n \tau_{*}^{\alpha}\right]+1}^{[n \tau]}\left(F_{i}\left(\alpha_{1}\right)-F_{i}\left(\alpha_{2}\right)\right) .
\end{aligned}
$$

Now, from

$$
\begin{aligned}
F_{i}\left(\alpha_{1}\right)-F_{i}\left(\alpha_{2}\right)= & F_{i}\left(\alpha_{1}\right)-F_{i}\left(\alpha_{2}\right)-\mathbb{E}_{\alpha_{2}^{*}}\left[F_{i}\left(\alpha_{1}\right)-F_{i}\left(\alpha_{2}\right) \mid \mathscr{G}_{i-1}^{n}\right] \\
& +\operatorname{tr}\left(A_{i-1}^{-1}\left(\alpha_{1}\right) A_{i-1}\left(\alpha_{2}\right)-I_{d}\right)-\log \operatorname{det} A_{i-1}^{-1}\left(\alpha_{1}\right) A_{i-1}\left(\alpha_{2}\right) \\
& -\operatorname{tr}\left(\left(A_{i-1}^{-1}\left(\alpha_{1}\right)-A_{i-1}^{-1}\left(\alpha_{2}\right)\right)\left(A_{i-1}\left(\alpha_{2}\right)-h^{-1} \mathbb{E}_{\alpha_{2}^{*}}\left[\left(\Delta X_{i}\right)^{\otimes 2} \mid \mathscr{G}_{i-1}^{n}\right]\right)\right),
\end{aligned}
$$

we obtain

$$
\begin{aligned}
& \Phi_{n}\left(\tau: \alpha_{1}, \alpha_{2}\right)-\Phi_{n}\left(\tau_{*}^{\alpha}: \alpha_{1}, \alpha_{2}\right) \\
& =\sum_{i=\left[n \tau_{*}^{\alpha}\right]+1}^{[n \tau]}\left(F_{i}\left(\alpha_{1}\right)-F_{i}\left(\alpha_{2}\right)-\mathbb{E}_{\alpha_{2}^{*}}\left[F_{i}\left(\alpha_{1}\right)-F_{i}\left(\alpha_{2}\right) \mid \mathscr{G}_{i-1}^{n}\right]\right) \\
& \quad+\sum_{i=\left[n \tau_{*}^{\alpha}\right]+1}^{[n \tau]}\left(\operatorname{tr}\left(A_{i-1}^{-1}\left(\alpha_{1}\right) A_{i-1}\left(\alpha_{2}\right)-I_{d}\right)-\log \operatorname{det} A_{i-1}^{-1}\left(\alpha_{1}\right) A_{i-1}\left(\alpha_{2}\right)\right) \\
& \quad-\sum_{i=\left[n \tau_{*}^{\alpha}\right]+1}^{n \tau]} \operatorname{tr}\left(\left(A_{i-1}^{-1}\left(\alpha_{1}\right)-A_{i-1}^{-1}\left(\alpha_{2}\right)\right)\left(A_{i-1}\left(\alpha_{2}\right)-h^{-1} \mathbb{E}_{\alpha_{2}^{*}}\left[\left(\Delta X_{i}\right)^{\otimes 2} \mid \mathscr{G}_{i-1}^{n}\right]\right)\right) \\
& =: \mathcal{M}_{n}^{\alpha}\left(\tau: \alpha_{1}, \alpha_{2}\right)+\mathcal{A}_{n}^{\alpha}\left(\tau: \alpha_{1}, \alpha_{2}\right)+\varrho_{n}^{\alpha}\left(\tau: \alpha_{1}, \alpha_{2}\right) .
\end{aligned}
$$

Let $D_{n, M}^{\alpha}=\left\{\tau \in[0,1] \mid n \vartheta_{\alpha}^{2}\left(\tau-\tau_{*}^{\alpha}\right)>M\right\}$. For all $\delta>0$, we have

$$
\begin{aligned}
P\left(n \vartheta_{\alpha}^{2}\left(\hat{\tau}_{n}-\tau_{*}^{\alpha}\right)>M\right) & \leq P\left(\inf _{\tau \in D_{n, M}^{\alpha}} \Phi_{n}\left(\tau: \hat{\alpha}_{1}, \hat{\alpha}_{2}\right) \leq \Phi_{n}\left(\tau_{*}^{\alpha}: \hat{\alpha}_{1}, \hat{\alpha}_{2}\right)\right) \\
& =P\left(\inf _{\tau \in D_{n, M}^{\alpha}}\left(\Phi_{n}\left(\tau: \hat{\alpha}_{1}, \hat{\alpha}_{2}\right)-\Phi_{n}\left(\tau_{*}^{\alpha}: \hat{\alpha}_{1}, \hat{\alpha}_{2}\right)\right) \leq 0\right) \\
& =P\left(\inf _{\tau \in D_{n, M}^{\alpha}}\left(\mathcal{M}_{n}^{\alpha}\left(\tau: \hat{\alpha}_{1}, \hat{\alpha}_{2}\right)+\mathcal{A}_{n}^{\alpha}\left(\tau: \hat{\alpha}_{1}, \hat{\alpha}_{2}\right)+\varrho_{n}^{\alpha}\left(\tau: \hat{\alpha}_{1}, \hat{\alpha}_{2}\right)\right) \leq 0\right)
\end{aligned}
$$




$$
\begin{aligned}
\leq & P\left(\inf _{\tau \in D_{n, M}^{\alpha}} \frac{\mathcal{M}_{n}^{\alpha}\left(\tau: \hat{\alpha}_{1}, \hat{\alpha}_{2}\right)+\mathcal{A}_{n}^{\alpha}\left(\tau: \hat{\alpha}_{1}, \hat{\alpha}_{2}\right)+\varrho_{n}^{\alpha}\left(\tau: \hat{\alpha}_{1}, \hat{\alpha}_{2}\right)}{\vartheta_{\alpha}^{2}\left([n \tau]-\left[n \tau_{*}^{\alpha}\right]\right)} \leq 0\right) \\
\leq & P\left(\inf _{\tau \in D_{n, M}^{\alpha}} \frac{\mathcal{M}_{n}^{\alpha}\left(\tau: \hat{\alpha}_{1}, \hat{\alpha}_{2}\right)}{\vartheta_{\alpha}^{2}\left([n \tau]-\left[n \tau_{*}^{\alpha}\right]\right)} \leq-\delta\right)+P\left(\inf _{\tau \in D_{n, M}^{\alpha}} \frac{\mathcal{A}_{n}^{\alpha}\left(\tau: \hat{\alpha}_{1}, \hat{\alpha}_{2}\right)}{\vartheta_{\alpha}^{2}\left([n \tau]-\left[n \tau_{*}^{\alpha}\right]\right)} \leq 2 \delta\right) \\
& +P\left(\inf _{\tau \in D_{n, M}^{\alpha}} \frac{\varrho_{n}^{\alpha}\left(\tau: \hat{\alpha}_{1}, \hat{\alpha}_{2}\right)}{\vartheta_{\alpha}^{2}\left([n \tau]-\left[n \tau_{*}^{\alpha}\right]\right)} \leq-\delta\right) \\
\leq & P\left(\sup _{\tau \in D_{n, M}^{\alpha}} \frac{\left|\mathcal{M}_{n}^{\alpha}\left(\tau: \hat{\alpha}_{1}, \hat{\alpha}_{2}\right)\right|}{\vartheta_{\alpha}^{2}\left([n \tau]-\left[n \tau_{*}^{\alpha}\right]\right)} \geq \delta\right)+P\left(\inf _{\tau \in D_{n, M}^{\alpha}} \frac{\mathcal{A}_{n}^{\alpha}\left(\tau: \hat{\alpha}_{1}, \hat{\alpha}_{2}\right)}{\vartheta_{\alpha}^{2}\left([n \tau]-\left[n \tau_{*}^{\alpha}\right]\right)} \leq 2 \delta\right) \\
& +P\left(\sup _{\tau \in D_{n, M}^{\alpha}} \frac{\left|\varrho_{n}^{\alpha}\left(\tau: \hat{\alpha}_{1}, \hat{\alpha}_{2}\right)\right|}{\vartheta_{\alpha}^{2}\left([n \tau]-\left[n \tau_{*}^{\alpha}\right]\right)} \geq \delta\right) \\
= & P_{1, n}^{\alpha}+P_{2, n}^{\alpha}+P_{3, n}^{\alpha} .
\end{aligned}
$$

[i] Evaluation of $P_{1, n}^{\alpha}$. Choose $\epsilon>0$ as you like. Let $\mathcal{O}_{\alpha}$ be an open neighborhood of $\alpha$. Because $\partial_{\alpha} F_{i}(\alpha)$ is continuous with respect to $\alpha \in \Theta_{A}$, we can choose $\bar{\alpha} \in \mathcal{O}_{\hat{\alpha}_{2}}$ so that

$$
\begin{aligned}
\mathcal{M}_{n}^{\alpha}\left(\tau: \hat{\alpha}_{1}, \hat{\alpha}_{2}\right) & =\sum_{i=\left[n \tau_{*}^{\alpha}\right]+1}^{[n \tau]}\left(F_{i}\left(\hat{\alpha}_{1}\right)-F_{i}\left(\hat{\alpha}_{2}\right)-\left.\mathbb{E}_{\alpha_{2}^{*}}\left[F_{i}\left(\alpha_{1}\right)-F_{i}\left(\alpha_{2}\right) \mid \mathscr{G}_{i-1}^{n}\right]\right|_{\alpha_{k}=\hat{\alpha}_{k}}\right) \\
& =\sum_{i=\left[n \tau_{*}^{\alpha}\right]+1}^{[n \tau]}\left(\partial_{\alpha} F_{i}(\bar{\alpha})-\left.\mathbb{E}_{\alpha_{2}^{*}}\left[\partial_{\alpha} F_{i}(\alpha) \mid \mathscr{G}_{i-1}^{n}\right]\right|_{\alpha=\bar{\alpha}}\right)\left(\hat{\alpha}_{1}-\hat{\alpha}_{2}\right) .
\end{aligned}
$$

If $\hat{\alpha}_{k} \in \mathcal{O}_{\alpha_{0}}$, then

$$
\begin{aligned}
\left|\mathcal{M}_{n}^{\alpha}\left(\tau: \hat{\alpha}_{1}, \hat{\alpha}_{2}\right)\right| & \leq\left|\sum_{i=\left[n \tau_{*}^{\alpha}\right]+1}^{[n \tau]}\left(\partial_{\alpha} F_{i}(\bar{\alpha})-\left.\mathbb{E}_{\alpha_{2}^{*}}\left[\partial_{\alpha} F_{i}(\alpha) \mid \mathscr{G}_{i-1}^{n}\right]\right|_{\alpha=\bar{\alpha}}\right)\right|\left|\hat{\alpha}_{1}-\hat{\alpha}_{2}\right| \\
& \leq \sup _{\alpha \in \Theta_{A}}\left|\sum_{i=\left[n \tau_{*}^{\alpha}\right]+1}^{[n \tau]}\left(\partial_{\alpha} F_{i}(\alpha)-\mathbb{E}_{\alpha_{2}^{*}}\left[\partial_{\alpha} F_{i}(\alpha) \mid \mathscr{G}_{i-1}^{n}\right]\right)\right|\left|\hat{\alpha}_{1}-\hat{\alpha}_{2}\right| \\
& =: \sup _{\alpha \in \Theta_{A}}\left|\mathbb{M}_{n}^{\alpha}(\tau: \alpha)\right|\left|\hat{\alpha}_{1}-\hat{\alpha}_{2}\right| .
\end{aligned}
$$

Hence we have

$$
\begin{aligned}
P_{1, n}^{\alpha}= & P\left(\sup _{\tau \in D_{n, M}^{\alpha}} \frac{\left|\mathcal{M}_{n}^{\alpha}\left(\tau: \hat{\alpha}_{1}, \hat{\alpha}_{2}\right)\right|}{\vartheta_{\alpha}^{2}\left([n \tau]-\left[n \tau_{*}^{\alpha}\right]\right)} \geq \delta\right) \\
\leq P & \left(\sup _{\tau \in D_{n, M}^{\alpha}} \frac{\left|\mathcal{M}_{n}^{\alpha}\left(\tau: \hat{\alpha}_{1}, \hat{\alpha}_{2}\right)\right|}{\vartheta_{\alpha}^{2}\left([n \tau]-\left[n \tau_{*}^{\alpha}\right]\right)} \geq \delta,\left|\hat{\alpha}_{1}-\hat{\alpha}_{2}\right| \leq 2 \vartheta_{\alpha}, \hat{\alpha}_{1}, \hat{\alpha}_{2} \in \mathcal{O}_{\alpha_{0}}\right) \\
& +P\left(\left|\hat{\alpha}_{1}-\hat{\alpha}_{2}\right|>2 \vartheta_{\alpha}\right)+P\left(\hat{\alpha}_{1} \notin \mathcal{O}_{\alpha_{0}}\right)+P\left(\hat{\alpha}_{2} \notin \mathcal{O}_{\alpha_{0}}\right) \\
\leq & P\left(\sup _{\tau \in D_{n, M}^{\alpha}} \frac{\sup _{\alpha \in \Theta_{A}}\left|\mathbb{M}_{n}^{\alpha}(\tau: \alpha)\right|}{\vartheta_{\alpha}^{2}\left([n \tau]-\left[n \tau_{*}^{\alpha}\right]\right)}\left|\hat{\alpha}_{1}-\hat{\alpha}_{2}\right| \geq \delta,\left|\hat{\alpha}_{1}-\hat{\alpha}_{2}\right| \leq 2 \vartheta_{\alpha}\right) \\
& +P\left(\left|\hat{\alpha}_{1}-\hat{\alpha}_{2}\right|>2 \vartheta_{\alpha}\right)+P\left(\hat{\alpha}_{1} \notin \mathcal{O}_{\alpha_{0}}\right)+P\left(\hat{\alpha}_{2} \notin \mathcal{O}_{\alpha_{0}}\right) \\
\leq & P\left(\sup _{\tau \in D_{n, M}^{\alpha}} \frac{2 \sup _{\alpha \in \Theta_{A}}\left|\mathbb{M}_{n}^{\alpha}(\tau: \alpha)\right|}{\vartheta_{\alpha}\left([n \tau]-\left[n \tau_{*}^{\alpha}\right]\right)} \geq \delta\right) \\
& +P\left(\left|\hat{\alpha}_{1}-\hat{\alpha}_{2}\right|>2 \vartheta_{\alpha}\right)+P\left(\hat{\alpha}_{1} \notin \mathcal{O}_{\alpha_{0}}\right)+P\left(\hat{\alpha}_{2} \notin \mathcal{O}_{\alpha_{0}}\right) .
\end{aligned}
$$

By the uniform version on the Hájek-Renyi inequality in Lemma 2 of Iacus and Yoshida (2012), we obtain

$$
P\left(\sup _{\tau \in D_{n, M}^{\alpha}} \frac{2 \sup _{\alpha \in \Theta_{A}}\left|\mathbb{M}_{n}^{\alpha}(\tau: \alpha)\right|}{\vartheta_{\alpha}\left([n \tau]-\left[n \tau_{*}^{\alpha}\right]\right)} \geq \delta\right)
$$




$$
\begin{aligned}
& =P\left(\sup _{\tau \in D_{n, M}^{\alpha}} \frac{2 \sup _{\alpha \in \Theta_{A}}\left|\mathbb{M}_{n}^{\alpha}(\tau: \alpha)\right|}{[n \tau]-\left[n \tau_{*}^{\alpha}\right]} \geq \delta \vartheta_{\alpha}\right) \\
& \leq P\left(\max _{j>M / \vartheta_{\alpha}^{2}-1} \frac{2}{j} \sup _{\alpha}\left|\sum_{i=\left[n \tau_{*}^{\alpha}\right]+1}^{\left[n \tau_{*}^{\alpha}\right]+j}\left(\partial_{\alpha} F_{i}(\alpha)-\mathbb{E}\left[\partial_{\alpha} F_{i}(\alpha) \mid \mathscr{G}_{i-1}^{n}\right]\right)\right| \geq \delta \vartheta_{\alpha}\right) \\
& \leq \sum_{j>M / \vartheta_{\alpha}^{2}-1} \frac{C}{\left(\delta \vartheta_{\alpha} j\right)^{2}} \\
& \leq \frac{C^{\prime}}{\left(\delta \vartheta_{\alpha}\right)^{2}} \frac{\vartheta_{\alpha}^{2}}{M}=\frac{C^{\prime}}{\delta^{2} M}=: \gamma_{\alpha}(M),
\end{aligned}
$$

Noting that if $\left|\hat{\alpha}_{k}-\alpha_{k}^{*}\right| \leq \vartheta_{\alpha} / 2$ holds, then $\left|\hat{\alpha}_{1}-\hat{\alpha}_{2}\right| \leq\left|\hat{\alpha}_{1}-\alpha_{1}^{*}\right|+\left|\alpha_{1}^{*}-\alpha_{2}^{*}\right|+\left|\hat{\alpha}_{2}-\alpha_{2}^{*}\right| \leq 2 \vartheta_{\alpha}$, i.e.,

$$
\left\{\left|\hat{\alpha}_{1}-\hat{\alpha}_{2}\right|>2 \vartheta_{\alpha}\right\} \subset\left\{\left|\hat{\alpha}_{1}-\alpha_{1}^{*}\right|>\frac{\vartheta_{\alpha}}{2}\right\} \cup\left\{\left|\hat{\alpha}_{2}-\alpha_{2}^{*}\right|>\frac{\vartheta_{\alpha}}{2}\right\},
$$

and for sufficiently large $n$ so that $P\left(\left|\hat{\alpha}_{k}-\alpha_{k}^{*}\right|>\vartheta_{\alpha} / 2\right)<\epsilon / 2$ because of $\vartheta_{\alpha}^{-1}\left(\hat{\alpha}_{k}-\alpha_{k}^{*}\right)=o_{p}(1)$, we see

$$
P\left(\left|\hat{\alpha}_{1}-\hat{\alpha}_{2}\right|>2 \vartheta_{\alpha}\right) \leq P\left(\left|\hat{\alpha}_{1}-\alpha_{1}^{*}\right|>\frac{\vartheta_{\alpha}}{2}\right)+P\left(\left|\hat{\alpha}_{2}-\alpha_{2}^{*}\right|>\frac{\vartheta_{\alpha}}{2}\right)<\epsilon .
$$

From [C6-I] and [A2-I], we have $\hat{\alpha}_{k} \stackrel{p}{\longrightarrow} \alpha_{0}$ as $n \rightarrow \infty$ and $P\left(\hat{\alpha}_{k} \notin \mathcal{O}_{\alpha_{0}}\right)<\epsilon$ for large $n$. Therefore, from 7.20)-7.22 and this, we have $P_{1, n}^{\alpha} \leq \gamma_{\alpha}(M)+3 \epsilon$ for large $n$.

[ii] Evaluation of $P_{2, n}^{\alpha}$. If $\hat{\alpha}_{k} \in \mathcal{O}_{\alpha_{0}}$, then we have

$$
\begin{aligned}
\operatorname{tr}( & \left.A_{i-1}^{-1}\left(\hat{\alpha}_{1}\right) A_{i-1}\left(\hat{\alpha}_{2}\right)-I_{d}\right)-\log \operatorname{det} A_{i-1}^{-1}\left(\hat{\alpha}_{1}\right) A_{i-1}\left(\hat{\alpha}_{2}\right) \\
=\operatorname{tr} & \left(A_{i-1}^{-1}\left(\hat{\alpha}_{2}\right) A_{i-1}\left(\hat{\alpha}_{2}\right)-I_{d}\right)-\log \operatorname{det} A_{i-1}^{-1}\left(\hat{\alpha}_{2}\right) A_{i-1}\left(\hat{\alpha}_{2}\right) \\
& +\left.\partial_{\alpha}\left(\operatorname{tr}\left(A_{i-1}^{-1}(\alpha) A_{i-1}\left(\hat{\alpha}_{2}\right)\right)-\log \operatorname{det} A_{i-1}^{-1}(\alpha) A_{i-1}\left(\hat{\alpha}_{2}\right)\right)\right|_{\alpha=\hat{\alpha}_{2}}\left(\hat{\alpha}_{1}-\hat{\alpha}_{2}\right) \\
& +\left.\frac{1}{2} \partial_{\alpha}^{2}\left(\operatorname{tr}\left(A_{i-1}^{-1}(\alpha) A_{i-1}\left(\hat{\alpha}_{2}\right)\right)-\log \operatorname{det} A_{i-1}^{-1}(\alpha) A_{i-1}\left(\hat{\alpha}_{2}\right)\right)\right|_{\alpha=\hat{\alpha}_{2}} \otimes\left(\hat{\alpha}_{1}-\hat{\alpha}_{2}\right)^{\otimes 2} \\
& +\left.\frac{1}{3 !} \partial_{\alpha}^{3}\left(\operatorname{tr}\left(A_{i-1}^{-1}(\alpha) A_{i-1}\left(\hat{\alpha}_{2}\right)\right)-\log \operatorname{det} A_{i-1}^{-1}(\alpha) A_{i-1}\left(\hat{\alpha}_{2}\right)\right)\right|_{\alpha=\hat{\alpha}_{2}} \otimes\left(\hat{\alpha}_{1}-\hat{\alpha}_{2}\right)^{\otimes 3} \\
& +\left.\int_{0}^{1} \frac{(1-u)^{3}}{3 !} \partial_{\alpha}^{4}\left(\operatorname{tr}\left(A_{i-1}^{-1}(\alpha) A_{i-1}\left(\hat{\alpha}_{2}\right)\right)-\log \operatorname{det} A_{i-1}^{-1}(\alpha) A_{i-1}\left(\hat{\alpha}_{2}\right)\right)\right|_{\alpha=\hat{\alpha}_{2}+u\left(\hat{\alpha}_{1}-\hat{\alpha}_{2}\right)} \mathrm{d} u \otimes\left(\hat{\alpha}_{1}-\hat{\alpha}_{2}\right)^{\otimes 4} \\
=\frac{1}{2} \Xi_{i-1}^{\alpha}\left(\hat{\alpha}_{2}\right) \otimes\left(\hat{\alpha}_{1}-\hat{\alpha}_{2}\right)^{\otimes 2} & \quad+\left.\frac{1}{3 !} \partial_{\alpha}^{3}\left(\operatorname{tr}\left(A_{i-1}^{-1}(\alpha) A_{i-1}\left(\hat{\alpha}_{2}\right)\right)-\log \operatorname{det} A_{i-1}^{-1}(\alpha) A_{i-1}\left(\hat{\alpha}_{2}\right)\right)\right|_{\alpha=\hat{\alpha}_{2}} \otimes\left(\hat{\alpha}_{1}-\hat{\alpha}_{2}\right)^{\otimes 3} \mathrm{~d} u \otimes\left(\hat{\alpha}_{1}-\hat{\alpha}_{2}\right)^{\otimes 4} \\
& +\left.\int_{0}^{1} \frac{(1-u)^{3}}{3 !} \partial_{\alpha}^{4}\left(\operatorname{tr}\left(A_{i-1}^{-1}(\alpha) A_{i-1}\left(\hat{\alpha}_{2}\right)\right)-\log \operatorname{det} A_{i-1}^{-1}(\alpha) A_{i-1}\left(\hat{\alpha}_{2}\right)\right)\right|_{\alpha=\hat{\alpha}_{2}+u\left(\hat{\alpha}_{1}-\hat{\alpha}_{2}\right)} \\
=\frac{1}{2} \Xi_{i-1}^{\alpha}\left(\alpha_{0}\right) \otimes\left(\hat{\alpha}_{1}-\hat{\alpha}_{2}\right)^{\otimes 2}+\frac{1}{2} \partial_{\alpha} \Xi_{i-1}^{\alpha}\left(\alpha_{0}\right) \otimes\left(\hat{\alpha}_{1}-\hat{\alpha}_{2}\right)^{\otimes 2} \otimes\left(\hat{\alpha}_{2}-\alpha_{0}\right) & \mathrm{d} u \otimes\left(\hat{\alpha}_{1}-\hat{\alpha}_{2}\right)^{\otimes 4} \\
& +\frac{1}{2} \int_{0}^{1}(1-u) \partial_{\alpha}^{2} \Xi_{i-1}^{\alpha}\left(\alpha_{0}+u\left(\hat{\alpha}_{2}-\alpha_{0}\right)\right) \mathrm{d} u \otimes\left(\hat{\alpha}_{1}-\hat{\alpha}_{2}\right)^{\otimes 2} \otimes\left(\hat{\alpha}_{2}-\alpha_{0}\right)^{\otimes 2} \\
& +\left.\frac{1}{3 !} \partial_{\alpha}^{3}\left(\operatorname{tr}\left(A_{i-1}^{-1}(\alpha) A_{i-1}\left(\hat{\alpha}_{2}\right)\right)-\log \operatorname{det} A_{i-1}^{-1}(\alpha) A_{i-1}\left(\hat{\alpha}_{2}\right)\right)\right|_{\alpha=\hat{\alpha}_{2}} \otimes\left(\hat{\alpha}_{1}-\hat{\alpha}_{2}\right)^{\otimes 3} \\
& +\left.\int_{0}^{1} \frac{(1-u)^{3}}{3 !} \partial_{\alpha}^{4}\left(\operatorname{tr}\left(A_{i-1}^{-1}(\alpha) A_{i-1}\left(\hat{\alpha}_{2}\right)\right)-\log \operatorname{det} A_{i-1}^{-1}(\alpha) A_{i-1}\left(\hat{\alpha}_{2}\right)\right)\right|_{\alpha=\hat{\alpha}_{2}+u\left(\hat{\alpha}_{1}-\hat{\alpha}_{2}\right)} \\
\geq & \left.\frac{1}{2} \lambda_{1}\left[\Xi_{i-1}^{\alpha}\left(\alpha_{0}\right)\right]+r_{i-1}\right)\left|\hat{\alpha}_{1}-\hat{\alpha}_{2}\right|^{2},
\end{aligned}
$$


where $\lambda_{1}[N]$ denotes the minimum eigenvalue of a symmetric matrix $N$, and $r_{i-1}$ satisfies

$$
\sup _{\tau \in D_{n, M}^{\alpha}}\left|\frac{1}{[n \tau]-\left[n \tau_{*}^{\alpha}\right]} \sum_{i=\left[n \tau_{*}^{\alpha}\right]+1}^{[n \tau]} r_{i-1}\right|=o_{p}(1)
$$

from $[\mathbf{A} 1-\mathbf{I}]$ and $[\mathbf{A 2}-\mathbf{I}]$. Therefore we obtain

$$
\begin{aligned}
P_{2, n}^{\alpha}= & P\left(\inf _{\tau \in D_{n, M}^{\alpha}} \frac{\mathcal{A}_{n}^{\alpha}\left(\tau: \hat{\alpha}_{1}, \hat{\alpha}_{2}\right)}{\vartheta_{\alpha}^{2}\left([n \tau]-\left[n \tau_{*}^{\alpha}\right]\right)} \leq 2 \delta\right) \\
\leq & P\left(\inf _{\tau \in D_{n, M}^{\alpha}} \frac{\mathcal{A}_{n}^{\alpha}\left(\tau: \hat{\alpha}_{1}, \hat{\alpha}_{2}\right)}{\vartheta_{\alpha}^{2}\left([n \tau]-\left[n \tau_{*}^{\alpha}\right]\right)} \leq 2 \delta,\left|\hat{\alpha}_{1}-\hat{\alpha}_{2}\right| \geq \frac{\vartheta_{\alpha}}{2}, \hat{\alpha}_{k} \in \mathcal{O}_{\alpha_{0}}\right) \\
& +P\left(\left|\hat{\alpha}_{1}-\hat{\alpha}_{2}\right|<\frac{\vartheta_{\alpha}}{2}\right)+P\left(\hat{\alpha}_{1} \notin \mathcal{O}_{\alpha_{0}}\right)+P\left(\hat{\alpha}_{2} \notin \mathcal{O}_{\alpha_{0}}\right) \\
\leq & P\left(\inf _{\tau \in D_{n, M}^{\alpha}} \frac{1}{\vartheta_{\alpha}^{2}\left([n \tau]-\left[n \tau_{*}^{\alpha}\right]\right)} \sum_{i=\left[n \tau_{*}^{\alpha}\right]+1}^{[n \tau]}\left(\frac{1}{2} \lambda_{1}\left[\Xi_{i-1}^{\alpha}\left(\alpha_{0}\right)\right]+r_{i-1}\right)\left|\hat{\alpha}_{1}-\hat{\alpha}_{2}\right|^{2} \leq 2 \delta,\left|\hat{\alpha}_{1}-\hat{\alpha}_{2}\right| \geq \frac{\vartheta_{\alpha}}{2}\right) \\
& +P\left(\left|\hat{\alpha}_{1}-\hat{\alpha}_{2}\right|<\frac{\vartheta_{\alpha}}{2}\right)+P\left(\hat{\alpha}_{1} \notin \mathcal{O}_{\alpha_{0}}\right)+P\left(\hat{\alpha}_{2} \notin \mathcal{O}_{\alpha_{0}}\right) \\
\leq & P\left(\inf _{\tau \in D_{n, M}^{\alpha}} \frac{1}{[n \tau]-\left[n \tau_{*}^{\alpha}\right]} \sum_{i=\left[n \tau_{*}^{\alpha}\right]+1}^{[n \tau]}\left(\frac{1}{2} \lambda_{1}\left[\Xi_{i-1}^{\alpha}\left(\alpha_{0}\right)\right]+r_{i-1}\right) \leq 8 \delta\right) \\
& +P\left(\left|\hat{\alpha}_{1}-\hat{\alpha}_{2}\right|<\frac{\vartheta_{\alpha}}{2}\right)+P\left(\hat{\alpha}_{1} \notin \mathcal{O}_{\alpha_{0}}\right)+P\left(\hat{\alpha}_{2} \notin \mathcal{O}_{\alpha_{0}}\right) .
\end{aligned}
$$

According to [A2-I], if we set

$$
\delta=\frac{1}{19} \int_{\mathbb{R}^{d}} \lambda_{1}\left[\Xi^{\alpha}\left(x, \alpha_{0}\right)\right] \mathrm{d} \mu_{\alpha_{0}}(x)>0,
$$

then for large $n$,

$$
\begin{aligned}
& P\left(\inf _{\tau \in D_{n, M}^{\alpha}} \frac{1}{[n \tau]-\left[n \tau_{*}^{\alpha}\right]} \sum_{i=\left[n \tau_{*}^{\alpha}\right]+1}^{[n \tau]}\left(\frac{1}{2} \lambda_{1}\left[\Xi_{i-1}^{\alpha}\left(\alpha_{0}\right)\right]+r_{i-1}\right) \leq 8 \delta\right) \\
& \leq P\left(\inf _{\tau \in D_{n, M}^{\alpha}} \frac{1}{[n \tau]-\left[n \tau_{*}^{\alpha}\right]} \sum_{i=\left[n \tau_{*}^{\alpha}\right]+1}^{[n \tau]} \frac{1}{2} \lambda_{1}\left[\Xi_{i-1}^{\alpha}\left(\alpha_{0}\right)\right] \leq 9 \delta\right)+P\left(\inf _{\tau \in D_{n, M}^{\alpha}} \frac{1}{[n \tau]-\left[n \tau_{*}^{\alpha}\right]} \sum_{i=\left[n \tau_{*}^{\alpha}\right]+1}^{[n \tau]} r_{i-1} \leq-\delta\right) \\
& \leq P\left(\inf _{\tau \in D_{n, M}^{\alpha}} \frac{1}{[n \tau]-\left[n \tau_{*}^{\alpha}\right]} \sum_{i=\left[n \tau_{*}^{\alpha}\right]+1}^{[n \tau]} \lambda_{1}\left[\Xi_{i-1}^{\alpha}\left(\alpha_{0}\right)\right] \leq 18 \delta\right)+P\left(\sup _{\tau \in D_{n, M}^{\alpha}}\left|\frac{1}{[n \tau]-\left[n \tau_{*}^{\alpha}\right]} \sum_{i=\left[n \tau_{*}^{\alpha}\right]+1}^{[n \tau]} r_{i-1}\right| \geq \delta\right) \\
& \leq P\left(\inf _{\tau \in D_{n, M}^{\alpha}}\left(\frac{1}{[n \tau]-\left[n \tau_{*}^{\alpha}\right]} \sum_{i=\left[n \tau_{*}^{\alpha}\right]+1}^{[n \tau]} \lambda_{1}\left[\Xi_{i-1}^{\alpha}\left(\alpha_{0}\right)\right]-19 \delta\right) \leq-\delta\right)+\epsilon \\
& \leq P\left(\sup _{\tau \in D_{n, M}^{\alpha}}\left|\frac{1}{[n \tau]-\left[n \tau_{*}^{\alpha}\right]} \sum_{i=\left[n \tau_{*}^{\alpha}\right]+1}^{n \tau]} \lambda_{1}\left[\Xi_{i-1}^{\alpha}\left(\alpha_{0}\right)\right]-19 \delta\right| \geq \delta\right)+\epsilon \\
& \leq P\left(\sup _{k>M / \vartheta_{\alpha}^{2}-1}\left|\frac{1}{k} \sum_{i=\left[n \tau_{*}^{\alpha}\right]+1}^{\left[n \tau_{*}^{\alpha}\right]+k} \lambda_{1}\left[\Xi_{i-1}^{\alpha}\left(\alpha_{0}\right)\right]-19 \delta\right| \geq \delta\right)+\epsilon \\
& \leq P\left(\max _{\left[n^{1 / r}\right] \leq k \leq n}\left|\frac{1}{k} \sum_{i=\left[n \tau_{*}^{\alpha}\right]+1}^{\left[n \tau_{*}^{\alpha}\right]+k} \lambda_{1}\left[\Xi_{i-1}^{\alpha}\left(\alpha_{0}\right)\right]-19 \delta\right| \geq \delta\right)+\epsilon \\
& \leq 2 \epsilon .
\end{aligned}
$$


Noting that if $\left|\hat{\alpha}_{k}-\alpha_{k}^{*}\right| \leq \vartheta_{\alpha} / 4$ holds, then $\vartheta_{\alpha}=\left|\alpha_{1}^{*}-\alpha_{2}^{*}\right| \leq\left|\alpha_{1}^{*}-\hat{\alpha}_{1}\right|+\left|\hat{\alpha}_{1}-\hat{\alpha}_{2}\right|+\left|\hat{\alpha}_{2}-\alpha_{2}^{*}\right| \leq\left|\hat{\alpha}_{1}-\hat{\alpha}_{2}\right|+\vartheta_{\alpha} / 2$, i.e.,

$$
\left\{\left|\hat{\alpha}_{1}-\hat{\alpha}_{2}\right|<\frac{\vartheta_{\alpha}}{2}\right\} \subset\left\{\left|\hat{\alpha}_{1}-\alpha_{1}^{*}\right|>\frac{\vartheta_{\alpha}}{4}\right\} \cup\left\{\left|\hat{\alpha}_{2}-\alpha_{2}^{*}\right|>\frac{\vartheta_{\alpha}}{4}\right\}
$$

and for sufficiently large $n$ so that $P\left(\left|\hat{\alpha}_{k}-\alpha_{k}^{*}\right|>\vartheta_{\alpha} / 4\right)<\epsilon / 2$ because of $\vartheta_{\alpha}^{-1}\left(\hat{\alpha}_{k}-\alpha_{k}^{*}\right)=o_{p}(1)$, we see

$$
P\left(\left|\hat{\alpha}_{1}-\hat{\alpha}_{2}\right|<\frac{\vartheta_{\alpha}}{2}\right) \leq P\left(\left|\hat{\alpha}_{1}-\alpha_{1}^{*}\right|>\frac{\vartheta_{\alpha}}{4}\right)+P\left(\left|\hat{\alpha}_{2}-\alpha_{2}^{*}\right|>\frac{\vartheta_{\alpha}}{4}\right)<\epsilon .
$$

Therefore, from (7.23)-77.25), we obtain $P_{2, n}^{\alpha} \leq 5 \epsilon$ for large $n$.

[iii] Evaluation of $P_{3, n}^{\alpha}$. We have, for large $n$,

$$
\begin{aligned}
& \operatorname{tr}\left(\left(A_{i-1}^{-1}\left(\hat{\alpha}_{1}\right)-A_{i-1}^{-1}\left(\hat{\alpha}_{2}\right)\right)\left(A_{i-1}\left(\hat{\alpha}_{2}\right)-h^{-1} \mathbb{E}_{\alpha_{2}^{*}}\left[\left(\Delta X_{i}\right)^{\otimes 2} \mid \mathscr{G}_{i-1}^{n}\right]\right)\right) \\
& =\operatorname{tr}\left(\left(A_{i-1}^{-1}\left(\hat{\alpha}_{1}\right)-A_{i-1}^{-1}\left(\hat{\alpha}_{2}\right)\right)\left(A_{i-1}\left(\hat{\alpha}_{2}\right)-A_{i-1}\left(\alpha_{2}^{*}\right)+R_{i-1}(h, \theta)\right)\right) \\
& \leq \operatorname{tr}\left(\left(A_{i-1}^{-1}\left(\hat{\alpha}_{1}\right)-A_{i-1}^{-1}\left(\hat{\alpha}_{2}\right)\right)\left(A_{i-1}\left(\hat{\alpha}_{2}\right)-A_{i-1}\left(\alpha_{2}^{*}\right)\right)\right)+\left|A_{i-1}^{-1}\left(\hat{\alpha}_{1}\right)-A_{i-1}^{-1}\left(\hat{\alpha}_{2}\right)\right|\left|R_{i-1}(h, \theta)\right| \\
& \leq\left[\operatorname{tr}\left(\partial_{\alpha^{\ell_{1}}} A_{i-1}^{-1}\left(\hat{\alpha}_{2}\right)\left(A_{i-1}\left(\hat{\alpha}_{2}\right)-A_{i-1}\left(\alpha_{2}^{*}\right)\right)\right)\right]_{\ell_{1}}\left(\hat{\alpha}_{1}-\hat{\alpha}_{2}\right) \\
& +\int_{0}^{1}(1-u)\left[\operatorname{tr}\left(\partial_{\alpha^{\ell_{2}}} \partial_{\alpha^{\ell_{1}}} A_{i-1}^{-1}\left(\hat{\alpha}_{2}+u\left(\hat{\alpha}_{1}-\hat{\alpha}_{2}\right)\right)\left(A_{i-1}\left(\hat{\alpha}_{2}\right)-A_{i-1}\left(\alpha_{2}^{*}\right)\right)\right)\right]_{\ell_{1}, \ell_{2}} \mathrm{~d} u \otimes\left(\hat{\alpha}_{1}-\hat{\alpha}_{2}\right)^{\otimes 2} \\
& +\left|\int_{0}^{1} \partial_{\alpha} A_{i-1}^{-1}\left(\hat{\alpha}_{2}+u\left(\hat{\alpha}_{1}-\hat{\alpha}_{2}\right)\right) \mathrm{d} u\right|\left|R_{i-1}(h, \theta)\right|\left|\hat{\alpha}_{1}-\hat{\alpha}_{2}\right| \\
& =\left[\operatorname{tr}\left(\partial_{\alpha^{\ell_{1}}} A_{i-1}^{-1}\left(\hat{\alpha}_{2}\right) \partial_{\alpha^{\ell_{2}}} A_{i-1}\left(\alpha_{2}^{*}\right)\right)\right]_{\ell_{1}, \ell_{2}} \otimes\left(\hat{\alpha}_{1}-\hat{\alpha}_{2}\right) \otimes\left(\hat{\alpha}_{2}-\alpha_{2}^{*}\right) \\
& +\int_{0}^{1}(1-u)\left[\operatorname{tr}\left(\partial_{\alpha^{\ell_{1}}} A_{i-1}^{-1}\left(\hat{\alpha}_{2}\right) \partial_{\alpha^{\ell_{3}}} \partial_{\alpha^{\ell_{2}}} A_{i-1}\left(\alpha_{2}^{*}+u\left(\hat{\alpha}_{2}-\alpha_{2}^{*}\right)\right)\right)\right]_{\ell_{1}, \ell_{2}, \ell_{3}} \mathrm{~d} u \otimes\left(\hat{\alpha}_{1}-\hat{\alpha}_{2}\right) \otimes\left(\hat{\alpha}_{2}-\alpha_{2}^{*}\right)^{\otimes 2} \\
& +\int_{0}^{1}(1-v) \int_{0}^{1}(1-u)\left[\operatorname{tr}\left(\partial_{\alpha^{\ell_{2}}} \partial_{\alpha^{\ell_{1}}} A_{i-1}^{-1}\left(\hat{\alpha}_{2}+u\left(\hat{\alpha}_{1}-\hat{\alpha}_{2}\right)\right) \partial_{\alpha^{\ell_{3}}} A_{i-1}\left(\alpha_{2}^{*}+v\left(\hat{\alpha}_{2}-\alpha_{2}^{*}\right)\right)\right)\right]_{\ell_{1}, \ell_{2}, \ell_{3}} \mathrm{~d} u \mathrm{~d} v \\
& \otimes\left(\hat{\alpha}_{1}-\hat{\alpha}_{2}\right)^{\otimes 2} \otimes\left(\hat{\alpha}_{2}-\alpha_{2}^{*}\right) \\
& +\left|\int_{0}^{1} \partial_{\alpha} A_{i-1}^{-1}\left(\hat{\alpha}_{2}+u\left(\hat{\alpha}_{1}-\hat{\alpha}_{2}\right)\right) \mathrm{d} u\right|\left|R_{i-1}(h, \theta)\right|\left|\hat{\alpha}_{1}-\hat{\alpha}_{2}\right| \\
& =\Xi_{i-1}^{\alpha}\left(\alpha_{2}^{*}\right) \otimes\left(\hat{\alpha}_{1}-\hat{\alpha}_{2}\right) \otimes\left(\hat{\alpha}_{2}-\alpha_{2}^{*}\right) \\
& +\int_{0}^{1}\left[\operatorname{tr}\left(\partial_{\alpha^{\ell_{3}}} \partial_{\alpha^{\ell_{1}}} A_{i-1}^{-1}\left(\alpha_{2}^{*}+u\left(\hat{\alpha}_{2}-\alpha_{2}^{*}\right)\right) \partial_{\alpha^{\ell_{2}}} A_{i-1}\left(\alpha_{2}^{*}\right)\right)\right]_{\ell_{1}, \ell_{2}, \ell_{3}} \mathrm{~d} u \otimes\left(\hat{\alpha}_{1}-\hat{\alpha}_{2}\right) \otimes\left(\hat{\alpha}_{2}-\alpha_{2}^{*}\right)^{\otimes 2} \\
& +\int_{0}^{1}(1-u)\left[\operatorname{tr}\left(\partial_{\alpha^{\ell_{1}}} A_{i-1}^{-1}\left(\hat{\alpha}_{2}\right) \partial_{\alpha^{\ell_{3}}} \partial_{\alpha^{\ell_{2}}} A_{i-1}\left(\alpha_{2}^{*}+u\left(\hat{\alpha}_{2}-\alpha_{2}^{*}\right)\right)\right)\right]_{\ell_{1}, \ell_{2}, \ell_{3}} \mathrm{~d} u \otimes\left(\hat{\alpha}_{1}-\hat{\alpha}_{2}\right) \otimes\left(\hat{\alpha}_{2}-\alpha_{2}^{*}\right)^{\otimes 2} \\
& +\int_{0}^{1}(1-v) \int_{0}^{1}(1-u)\left[\operatorname{tr}\left(\partial_{\alpha^{\ell_{2}}} \partial_{\alpha^{\ell_{1}}} A_{i-1}^{-1}\left(\hat{\alpha}_{2}+u\left(\hat{\alpha}_{1}-\hat{\alpha}_{2}\right)\right) \partial_{\alpha^{\ell_{3}}} A_{i-1}\left(\alpha_{2}^{*}+v\left(\hat{\alpha}_{2}-\alpha_{2}^{*}\right)\right)\right)\right]_{\ell_{1}, \ell_{2}, \ell_{3}} \mathrm{~d} u \mathrm{~d} v \\
& \otimes\left(\hat{\alpha}_{1}-\hat{\alpha}_{2}\right)^{\otimes 2} \otimes\left(\hat{\alpha}_{2}-\alpha_{2}^{*}\right) \\
& +\left|\int_{0}^{1} \partial_{\alpha} A_{i-1}^{-1}\left(\hat{\alpha}_{2}+u\left(\hat{\alpha}_{1}-\hat{\alpha}_{2}\right)\right) \mathrm{d} u\right|\left|R_{i-1}(h, \theta)\right|\left|\hat{\alpha}_{1}-\hat{\alpha}_{2}\right| \\
& =\Xi_{i-1}^{\alpha}\left(\alpha_{0}\right) \otimes\left(\hat{\alpha}_{1}-\hat{\alpha}_{2}\right) \otimes\left(\hat{\alpha}_{2}-\alpha_{2}^{*}\right)+\int_{0}^{1} \partial_{\alpha} \Xi_{i-1}^{\alpha}\left(\alpha_{0}+u\left(\alpha_{2}^{*}-\alpha_{0}\right)\right) \mathrm{d} u \otimes\left(\hat{\alpha}_{1}-\hat{\alpha}_{2}\right) \otimes\left(\hat{\alpha}_{2}-\alpha_{2}^{*}\right) \otimes\left(\alpha_{2}^{*}-\alpha_{0}\right) \\
& +\int_{0}^{1}\left[\operatorname{tr}\left(\partial_{\alpha^{\ell_{3}}} \partial_{\alpha^{\ell_{1}}} A_{i-1}^{-1}\left(\alpha_{2}^{*}+u\left(\hat{\alpha}_{2}-\alpha_{2}^{*}\right)\right) \partial_{\alpha^{\ell_{2}}} A_{i-1}\left(\alpha_{2}^{*}\right)\right)\right]_{\ell_{1}, \ell_{2}, \ell_{3}} \mathrm{~d} u \otimes\left(\hat{\alpha}_{1}-\hat{\alpha}_{2}\right) \otimes\left(\hat{\alpha}_{2}-\alpha_{2}^{*}\right)^{\otimes 2} \\
& +\int_{0}^{1}(1-u)\left[\operatorname{tr}\left(\partial_{\alpha^{\ell_{1}}} A_{i-1}^{-1}\left(\hat{\alpha}_{2}\right) \partial_{\alpha^{\ell_{3}}} \partial_{\alpha^{\ell_{2}}} A_{i-1}\left(\alpha_{2}^{*}+u\left(\hat{\alpha}_{2}-\alpha_{2}^{*}\right)\right)\right)\right]_{\ell_{1}, \ell_{2}, \ell_{3}} \mathrm{~d} u \otimes\left(\hat{\alpha}_{1}-\hat{\alpha}_{2}\right) \otimes\left(\hat{\alpha}_{2}-\alpha_{2}^{*}\right)^{\otimes 2}
\end{aligned}
$$




$$
\begin{aligned}
& +\int_{0}^{1}(1-v) \int_{0}^{1}(1-u)\left[\operatorname{tr}\left(\partial_{\alpha^{\ell_{2}}} \partial_{\alpha^{\ell_{1}}} A_{i-1}^{-1}\left(\hat{\alpha}_{2}+u\left(\hat{\alpha}_{1}-\hat{\alpha}_{2}\right)\right) \partial_{\alpha^{\ell_{3}}} A_{i-1}\left(\alpha_{2}^{*}+v\left(\hat{\alpha}_{2}-\alpha_{2}^{*}\right)\right)\right)\right]_{\ell_{1}, \ell_{2}, \ell_{3}} \mathrm{~d} u \mathrm{~d} v \\
& \quad \otimes\left(\hat{\alpha}_{1}-\hat{\alpha}_{2}\right)^{\otimes 2} \otimes\left(\hat{\alpha}_{2}-\alpha_{2}^{*}\right) \\
& +\left|\int_{0}^{1} \partial_{\alpha} A_{i-1}^{-1}\left(\hat{\alpha}_{2}+u\left(\hat{\alpha}_{1}-\hat{\alpha}_{2}\right)\right) \mathrm{d} u\right|\left|R_{i-1}(h, \theta)\right|\left|\hat{\alpha}_{1}-\hat{\alpha}_{2}\right| \\
& \leq \Xi_{i-1}^{\alpha}\left(\alpha_{0}\right) \otimes\left(\hat{\alpha}_{1}-\hat{\alpha}_{2}\right) \otimes\left(\hat{\alpha}_{2}-\alpha_{2}^{*}\right) \\
& +\frac{\vartheta_{\alpha}^{2}}{\sqrt{n}}\left|\int_{0}^{1} \partial_{\alpha} \Xi_{i-1}^{\alpha}\left(\alpha_{0}+u\left(\alpha_{2}^{*}-\alpha_{0}\right)\right) \mathrm{d} u\right| \vartheta_{\alpha}^{-1}\left|\hat{\alpha}_{1}-\hat{\alpha}_{2}\right| \sqrt{n}\left|\hat{\alpha}_{2}-\alpha_{2}^{*}\right| \vartheta_{\alpha}^{-1}\left|\alpha_{2}^{*}-\alpha_{0}\right| \\
& +\frac{\vartheta_{\alpha}}{n}\left|\int_{0}^{1}\left[\operatorname{tr}\left(\partial_{\alpha^{\ell_{3}}} \partial_{\alpha^{\ell_{1}}} A_{i-1}^{-1}\left(\alpha_{2}^{*}+u\left(\hat{\alpha}_{2}-\alpha_{2}^{*}\right)\right) \partial_{\alpha^{\ell_{2}}} A_{i-1}\left(\alpha_{2}^{*}\right)\right)\right]_{\ell_{1}, \ell_{2}, \ell_{3}} \mathrm{~d} u\right| \vartheta_{\alpha}^{-1}\left|\hat{\alpha}_{1}-\hat{\alpha}_{2}\right|\left(\sqrt{n}\left|\hat{\alpha}_{2}-\alpha_{2}^{*}\right|\right)^{2} \\
& +\frac{\vartheta_{\alpha}}{n}\left|\int_{0}^{1}(1-u)\left[\operatorname{tr}\left(\partial_{\alpha^{\ell_{1}}} A_{i-1}^{-1}\left(\hat{\alpha}_{2}\right) \partial_{\alpha^{\ell_{3}}} \partial_{\alpha^{\ell_{2}}} A_{i-1}\left(\alpha_{2}^{*}+u\left(\hat{\alpha}_{2}-\alpha_{2}^{*}\right)\right)\right)\right]_{\ell_{1}, \ell_{2}, \ell_{3}} \mathrm{~d} u\right| \vartheta_{\alpha}^{-1}\left|\hat{\alpha}_{1}-\hat{\alpha}_{2}\right|\left(\sqrt{n}\left|\hat{\alpha}_{2}-\alpha_{2}^{*}\right|\right)^{2} \\
& +\frac{\vartheta_{\alpha}^{2}}{\sqrt{n}}\left|\int_{0}^{1}(1-v) \int_{0}^{1}(1-u)\left[\operatorname{tr}\left(\partial_{\alpha^{\ell_{2}}} \partial_{\alpha^{\ell_{1}}} A_{i-1}^{-1}\left(\hat{\alpha}_{2}+u\left(\hat{\alpha}_{1}-\hat{\alpha}_{2}\right)\right) \partial_{\alpha^{\ell_{3}}} A_{i-1}\left(\alpha_{2}^{*}+v\left(\hat{\alpha}_{2}-\alpha_{2}^{*}\right)\right)\right)\right]_{\ell_{1}, \ell_{2}, \ell_{3}} \mathrm{~d} u \mathrm{~d} v\right| \\
& \quad\left(\vartheta_{\alpha}^{-1}\left|\hat{\alpha}_{1}-\hat{\alpha}_{2}\right|\right)^{2} \sqrt{n}\left|\hat{\alpha}_{2}-\alpha_{2}^{*}\right| \\
& +h \vartheta_{\alpha}\left|\int_{0}^{1} \partial_{\alpha} A_{i-1}^{-1}\left(\hat{\alpha}_{2}+u\left(\hat{\alpha}_{1}-\hat{\alpha}_{2}\right)\right) \mathrm{d} u\right| R_{i-1}(1, \theta) \vartheta_{\alpha}^{-1}\left|\hat{\alpha}_{1}-\hat{\alpha}_{2}\right| \\
& \leq \frac{\vartheta_{\alpha}}{\sqrt{n}} \Xi_{i-1}^{\alpha}\left(\alpha_{0}\right) \otimes \vartheta_{\alpha}^{-1}\left(\hat{\alpha}_{1}-\hat{\alpha}_{2}\right) \otimes \sqrt{n}\left(\hat{\alpha}_{2}-\alpha_{2}^{*}\right)+\left(\frac{\vartheta_{\alpha}^{2}}{\sqrt{n}}+\frac{\vartheta_{\alpha}}{n}+h \vartheta_{\alpha}\right) R_{i-1}(1, \theta)
\end{aligned}
$$

and

$$
\begin{aligned}
& \sup _{\tau \in D_{n, M}^{\alpha}} \frac{\left|\varrho_{n}^{\alpha}\left(\tau: \hat{\alpha}_{1}, \hat{\alpha}_{2}\right)\right|}{\vartheta_{\alpha}^{2}\left([n \tau]-\left[n \tau_{*}^{\alpha}\right]\right)} \leq \frac{1}{\sqrt{n} \vartheta_{\alpha}} \sup _{\tau \in D_{n, M}^{\alpha}}\left|\frac{1}{[n \tau]-\left[n \tau_{*}^{\alpha}\right]} \sum_{i=\left[n \tau_{*}^{\alpha}\right]+1}^{[n \tau]} \Xi_{i-1}^{\alpha}\left(\alpha_{0}\right)\right| \vartheta_{\alpha}^{-1}\left|\hat{\alpha}_{1}-\hat{\alpha}_{2}\right| \sqrt{n}\left|\hat{\alpha}_{2}-\alpha_{2}^{*}\right| \\
& +\left(\frac{\vartheta_{\alpha}^{2}}{\sqrt{n}}+\frac{\vartheta_{\alpha}}{n}+h \vartheta_{\alpha}\right) \sup _{\tau \in D_{n, M}^{\alpha}} \frac{1}{\vartheta_{\alpha}^{2}\left([n \tau]-\left[n \tau_{*}^{\alpha}\right]\right)} \sum_{i=\left[n \tau_{*}^{\alpha}\right]+1}^{[n \tau]} R_{i-1}(1, \theta) \\
& \leq O_{p}\left(\frac{1}{\sqrt{n} \vartheta_{\alpha}}\right)+O_{p}\left(\sqrt{n} \vartheta_{\alpha}^{2}\right)+O_{p}\left(\vartheta_{\alpha}\right)+O_{p}\left(T \vartheta_{\alpha}\right)=o_{p}(1) \text {. }
\end{aligned}
$$

Hence, we see

$$
\begin{aligned}
P_{3, n}^{\alpha} & \leq P\left(\sup _{\tau \in D_{n, M}^{\alpha}} \frac{\left|\varrho_{n}^{\alpha}\left(\tau: \hat{\alpha}_{1}, \hat{\alpha}_{2}\right)\right|}{\vartheta_{\alpha}^{2}\left([n \tau]-\left[n \tau_{*}^{\alpha}\right]\right)} \geq \delta, \hat{\alpha}_{1}, \hat{\alpha}_{2} \in \mathcal{O}_{\alpha_{0}}\right)+P\left(\hat{\alpha}_{1} \notin \mathcal{O}_{\alpha_{0}}\right)+P\left(\hat{\alpha}_{2} \notin \mathcal{O}_{\alpha_{0}}\right) \\
& \leq 3 \epsilon
\end{aligned}
$$

for large $n$.

[iv] From the evaluations in Steps [i]-[iii], we have

$$
\varlimsup_{n \rightarrow \infty} P\left(n \vartheta_{\alpha}^{2}\left(\hat{\tau}_{n}^{\alpha}-\tau_{*}^{\alpha}\right)>M\right) \leq \gamma_{\alpha}(M)+11 \epsilon
$$

for any $M \geq 1$ and $\epsilon>0$. Therefore

$$
\varlimsup_{M \rightarrow \infty} \varlimsup_{n \rightarrow \infty} P\left(n \vartheta_{\alpha}^{2}\left(\hat{\tau}_{n}^{\alpha}-\tau_{*}^{\alpha}\right)>M\right) \leq 11 \epsilon
$$

Since, for $\tau<\tau_{*}^{\alpha}$,

$$
\begin{aligned}
& \Phi_{n}\left(\tau: \alpha_{1}, \alpha_{2}\right)-\Phi_{n}\left(\tau_{*}^{\alpha}: \alpha_{1}, \alpha_{2}\right) \\
& =\sum_{i=[n \tau]+1}^{\left[n \tau_{*}^{\alpha}\right]}\left(F_{i}\left(\alpha_{2}\right)-F_{i}\left(\alpha_{1}\right)-\mathbb{E}_{\alpha_{1}^{*}}\left[F_{i}\left(\alpha_{2}\right)-F_{i}\left(\alpha_{1}\right) \mid \mathscr{G}_{i-1}^{n}\right]\right)
\end{aligned}
$$




$$
\begin{aligned}
& +\sum_{i=[n \tau]+1}^{\left[n \tau_{*}^{\alpha}\right]}\left(\operatorname{tr}\left(A_{i-1}^{-1}\left(\alpha_{2}\right) A_{i-1}\left(\alpha_{1}\right)-I_{d}\right)-\log \operatorname{det} A_{i-1}^{-1}\left(\alpha_{2}\right) A_{i-1}\left(\alpha_{1}\right)\right) \\
& -\sum_{i=[n \tau]+1}^{\left[n \tau_{*}^{\alpha}\right]} \operatorname{tr}\left(\left(A_{i-1}^{-1}\left(\alpha_{2}\right)-A_{i-1}^{-1}\left(\alpha_{1}\right)\right)\left(A_{i-1}\left(\alpha_{1}\right)-h^{-1} \mathbb{E}_{\alpha_{1}^{*}}\left[\left(\Delta X_{i}\right)^{\otimes 2} \mid \mathscr{G}_{i-1}^{n}\right]\right)\right),
\end{aligned}
$$

we obtain, in the same way as above,

$$
\varlimsup_{M \rightarrow \infty} \varlimsup_{n \rightarrow \infty} P\left(n \vartheta_{\alpha}^{2}\left(\tau_{*}^{\alpha}-\hat{\tau}_{n}^{\alpha}\right)>M\right) \leq 11 \epsilon .
$$

We see, from 7.18, 7.26) and 7.27), $\varlimsup_{M \rightarrow \infty} \varlimsup_{n \rightarrow \infty} P\left(n \vartheta_{\alpha}^{2}\left|\hat{\tau}_{n}^{\alpha}-\tau_{*}^{\alpha}\right|>M\right) \leq 22 \epsilon$, which shows

$$
n \vartheta_{\alpha}^{2}\left(\hat{\tau}_{n}^{\alpha}-\tau_{*}^{\alpha}\right)=O_{p}(1) .
$$

From Lemmas 1.3 and 7.28 , we obtain

$$
n \vartheta_{\alpha}^{2}\left(\hat{\tau}_{n}^{\alpha}-\tau_{*}^{\alpha}\right) \stackrel{d}{\longrightarrow} \underset{v \in \mathbb{R}}{\operatorname{argmin}} \mathbb{F}(v) .
$$

This completes the proof of Theorem 1 .

Proof of Theorem 2, As with the proof of Theorem 1, it is sufficient to show

$$
\varlimsup_{M \rightarrow \infty} \varlimsup_{n \rightarrow \infty} P\left(n\left(\hat{\tau}_{n}^{\alpha}-\tau_{*}^{\alpha}\right)>M\right)=0 .
$$

Let $D_{n, M}^{\alpha}=\left\{\tau \in[0,1] \mid n\left(\tau-\tau_{*}^{\alpha}\right)>M\right\}$. For all $\delta>0$, we have

$$
\begin{aligned}
P\left(n\left(\hat{\tau}_{n}-\tau_{*}^{\alpha}\right)>M\right) \leq & P\left(\sup _{\tau \in D_{n, M}^{\alpha}} \frac{\left|\mathcal{M}_{n}^{\alpha}\left(\tau: \hat{\alpha}_{1}, \hat{\alpha}_{2}\right)\right|}{[n \tau]-\left[n \tau_{*}^{\alpha}\right]} \geq \delta\right)+P\left(\inf _{\tau \in D_{n, M}^{\alpha}} \frac{\mathcal{A}_{n}^{\alpha}\left(\tau: \hat{\alpha}_{1}, \hat{\alpha}_{2}\right)}{[n \tau]-\left[n \tau_{*}^{\alpha}\right]} \leq 2 \delta\right) \\
& +P\left(\sup _{\tau \in D_{n, M}^{\alpha}} \frac{\left|\varrho_{n}^{\alpha}\left(\tau: \hat{\alpha}_{1}, \hat{\alpha}_{2}\right)\right|}{[n \tau]-\left[n \tau_{*}^{\alpha}\right]} \geq \delta\right) \\
= & : P_{1, n}^{\alpha}+P_{2, n}^{\alpha}+P_{3, n}^{\alpha} .
\end{aligned}
$$

[i] Evaluation of $P_{1, n}^{\alpha}$. For large $n$, we have

$$
\begin{aligned}
P_{1, n}^{\alpha} & =P\left(\sup _{\tau \in D_{n, M}^{\alpha}} \frac{\left|\mathcal{M}_{n}^{\alpha}\left(\tau: \hat{\alpha}_{1}, \hat{\alpha}_{2}\right)\right|}{[n \tau]-\left[n \tau_{*}^{\alpha}\right]} \geq \delta\right) \\
& \leq P\left(\sup _{\tau \in D_{n, M}^{\alpha}} \frac{\left|\mathcal{M}_{n}^{\alpha}\left(\tau: \hat{\alpha}_{1}, \hat{\alpha}_{2}\right)\right|}{[n \tau]-\left[n \tau_{*}^{\alpha}\right]} \geq \delta, \hat{\alpha}_{1} \in \mathcal{O}_{\alpha_{1}^{*}}, \hat{\alpha}_{2} \in \mathcal{O}_{\alpha_{2}^{*}}\right)+P\left(\hat{\alpha}_{1} \notin \mathcal{O}_{\alpha_{1}^{*}}\right)+P\left(\hat{\alpha}_{2} \notin \mathcal{O}_{\alpha_{2}^{*}}\right) \\
& \leq P\left(\sup _{\tau \in D_{n, M}^{\alpha}} \frac{\sup _{\alpha_{k} \in \mathcal{O}_{\alpha_{k}^{*}}}\left|\mathcal{M}_{n}^{\alpha}\left(\tau: \alpha_{1}, \alpha_{2}\right)\right|}{[n \tau]-\left[n \tau_{*}^{\alpha}\right]} \geq \delta\right)+P\left(\hat{\alpha}_{1} \notin \mathcal{O}_{\alpha_{1}^{*}}\right)+P\left(\hat{\alpha}_{2} \notin \mathcal{O}_{\alpha_{2}^{*}}\right) .
\end{aligned}
$$

By the uniform version on the Hájek-Renyi inequality in Lemma 2 of Iacus and Yoshida (2012), we obtain

$$
\begin{aligned}
& P\left(\sup _{\tau \in D_{n, M}^{\alpha}} \frac{1}{[n \tau]-\left[n \tau_{*}^{\alpha}\right]} \sup _{\alpha_{k} \in \mathcal{O}_{\alpha_{k}^{*}}}\left|\mathcal{M}_{n}^{\alpha}\left(\tau: \alpha_{1}, \alpha_{2}\right)\right| \geq \delta\right) \\
& \leq P\left(\max _{j>M-1} \frac{1}{j} \sup _{\alpha_{k} \in \mathcal{O}_{\alpha_{k}^{*}}}\left|\sum_{i=\left[n \tau_{*}^{\alpha}\right]+1}^{\left[n \tau_{*}^{\alpha}\right]+j}\left(F_{i}\left(\alpha_{1}\right)-F_{i}\left(\alpha_{2}\right)-\mathbb{E}\left[F_{i}\left(\alpha_{1}\right)-F_{i}\left(\alpha_{2}\right) \mid \mathscr{G}_{i-1}^{n}\right]\right)\right| \geq \delta\right) \\
& \leq \sum_{j>M-1} \frac{C}{(\delta j)^{2}} \\
& \leq \frac{C^{\prime}}{\delta^{2} M}=: \gamma_{\alpha}(M) .
\end{aligned}
$$

From [C6-I], we have $P\left(\hat{\alpha}_{k} \notin \mathcal{O}_{\alpha_{k}^{*}}\right)<\epsilon$ for large $n$. Therefore $P_{1, n}^{\alpha} \leq \gamma_{\alpha}(M)+2 \epsilon$ for large $n$. 
[ii] Evaluation of $P_{2, n}^{\alpha}$. If $\hat{\alpha}_{k} \in \mathcal{O}_{\alpha_{k}^{*}}$, then there exists a positive constant $c$ independent of $i$ such that

$$
\begin{aligned}
\Gamma_{i-1}^{\alpha}\left(\hat{\alpha}_{1}, \hat{\alpha}_{2}\right) & =\Gamma_{i-1}^{\alpha}\left(\alpha_{1}^{*}, \alpha_{2}^{*}\right)+\left.\int_{0}^{1} \partial_{\left(\alpha_{1}, \alpha_{2}\right)} \Gamma_{i-1}^{\alpha}\left(\alpha_{1}, \alpha_{2}\right)\right|_{\alpha_{k}=\alpha_{k}^{*}+u\left(\hat{\alpha}_{k}-\alpha_{k}^{*}\right)} \mathrm{d} u\left(\begin{array}{c}
\hat{\alpha}_{1}-\alpha_{1}^{*} \\
\hat{\alpha}_{2}-\alpha_{2}^{*}
\end{array}\right) \\
& \geq \Gamma_{i-1}^{\alpha}\left(\alpha_{1}^{*}, \alpha_{2}^{*}\right)-c\left(\left|\hat{\alpha}_{1}-\alpha_{1}^{*}\right|+\left|\hat{\alpha}_{2}-\alpha_{2}^{*}\right|\right) .
\end{aligned}
$$

According to [B1-I], if we set

$$
\delta=\frac{1}{4} \inf _{x} \Gamma^{\alpha}\left(x, \alpha_{1}^{*}, \alpha_{2}^{*}\right)>0
$$

then for large $n$,

$$
\begin{aligned}
P_{2, n}^{\alpha} \leq & P\left(\inf _{\tau \in D_{n, M}^{\alpha}} \frac{\mathcal{A}_{n}^{\alpha}\left(\tau: \hat{\alpha}_{1}, \hat{\alpha}_{2}\right)}{[n \tau]-\left[n \tau_{*}^{\alpha}\right]} \leq 2 \delta, \hat{\alpha}_{1} \in \mathcal{O}_{\alpha_{1}^{*}}, \hat{\alpha}_{2} \in \mathcal{O}_{\alpha_{2}^{*}}\right)+P\left(\hat{\alpha}_{1} \notin \mathcal{O}_{\alpha_{1}^{*}}\right)+P\left(\hat{\alpha}_{2} \notin \mathcal{O}_{\alpha_{2}^{*}}\right) \\
\leq & P\left(\inf _{\tau \in D_{n, M}^{\alpha}} \frac{1}{[n \tau]-\left[n \tau_{*}^{\alpha}\right]} \sum_{i=\left[n \tau_{*}^{\alpha}\right]+1}^{[n \tau]}\left(\Gamma_{i-1}^{\alpha}\left(\alpha_{1}^{*}, \alpha_{2}^{*}\right)-c\left(\left|\hat{\alpha}_{1}-\alpha_{1}^{*}\right|+\left|\hat{\alpha}_{2}-\alpha_{2}^{*}\right|\right)\right) \leq 2 \delta\right) \\
& +P\left(\hat{\alpha}_{1} \notin \mathcal{O}_{\alpha_{1}^{*}}\right)+P\left(\hat{\alpha}_{2} \notin \mathcal{O}_{\alpha_{2}^{*}}\right) \\
\leq & P\left(\inf _{\tau \in D_{n, M}^{\alpha}} \frac{1}{[n \tau]-\left[n \tau_{*}^{\alpha}\right]} \sum_{i=\left[n \tau_{*}^{\alpha}\right]+1}^{[n \tau]} \Gamma_{i-1}^{\alpha}\left(\alpha_{1}^{*}, \alpha_{2}^{*}\right)-c\left(\left|\hat{\alpha}_{1}-\alpha_{1}^{*}\right|+\left|\hat{\alpha}_{2}-\alpha_{2}^{*}\right|\right) \leq 2 \delta\right) \\
& +P\left(\hat{\alpha}_{1} \notin \mathcal{O}_{\alpha_{1}^{*}}\right)+P\left(\hat{\alpha}_{2} \notin \mathcal{O}_{\alpha_{2}^{*}}\right) \\
\leq & P\left(\inf _{\tau \in D_{n, M}^{\alpha}} \frac{1}{[n \tau]-\left[n \tau_{*}^{\alpha}\right]} \sum_{i=\left[n \tau_{*}^{\alpha}\right]+1}^{[n \tau]} \Gamma_{i-1}^{\alpha}\left(\alpha_{1}^{*}, \alpha_{2}^{*}\right) \leq 3 \delta\right)+P\left(-c\left(\left|\hat{\alpha}_{1}-\alpha_{1}^{*}\right|+\left|\hat{\alpha}_{2}-\alpha_{2}^{*}\right|\right) \leq-\delta\right) \\
& \quad+P\left(\hat{\alpha}_{1} \notin \mathcal{O}_{\alpha_{1}^{*}}\right)+P\left(\hat{\alpha}_{2} \notin \mathcal{O}_{\alpha_{2}^{*}}\right) \\
\leq & P\left(\inf _{x} \Gamma^{\alpha}\left(x, \alpha_{1}^{*}, \alpha_{2}^{*}\right) \leq 3 \delta\right)+P\left(\left|\hat{\alpha}_{1}-\alpha_{1}^{*}\right|+\left|\hat{\alpha}_{2}-\alpha_{2}^{*}\right| \geq \frac{\delta}{c}\right)+P\left(\hat{\alpha}_{1} \notin \mathcal{O}_{\alpha_{1}^{*}}\right)+P\left(\hat{\alpha}_{2} \notin \mathcal{O}_{\alpha_{2}^{*}}\right) \\
\leq & 4 \epsilon
\end{aligned}
$$

thanks to

$$
P\left(\left|\hat{\alpha}_{1}-\alpha_{1}^{*}\right|+\left|\hat{\alpha}_{2}-\alpha_{2}^{*}\right| \geq \frac{\delta}{c}\right) \leq P\left(\left|\hat{\alpha}_{1}-\alpha_{1}^{*}\right| \geq \frac{\delta}{2 c}\right)+P\left(\left|\hat{\alpha}_{2}-\alpha_{2}^{*}\right| \geq \frac{\delta}{2 c}\right) \leq 2 \epsilon
$$

from [C6-I].

[iii] Evaluation of $P_{3, n}^{\alpha}$.

$$
\begin{aligned}
& \operatorname{tr}\left(\left(A_{i-1}^{-1}\left(\hat{\alpha}_{1}\right)-A_{i-1}^{-1}\left(\hat{\alpha}_{2}\right)\right)\left(A_{i-1}\left(\hat{\alpha}_{2}\right)-h^{-1} \mathbb{E}_{\alpha_{2}^{*}}\left[\left(\Delta X_{i}\right)^{\otimes 2} \mid \mathscr{G}_{i-1}^{n}\right]\right)\right) \\
& =\operatorname{tr}\left(\left(A_{i-1}^{-1}\left(\hat{\alpha}_{1}\right)-A_{i-1}^{-1}\left(\hat{\alpha}_{2}\right)\right)\left(A_{i-1}^{-1}\left(\hat{\alpha}_{2}\right)-A_{i-1}^{-1}\left(\alpha_{2}^{*}\right)-h Q_{i-1}\left(\theta^{*}\right)+R_{i-1}\left(h^{2}, \theta\right)\right)\right) \\
& \leq\left.\int_{0}^{1}\left[\operatorname{tr}\left(\left(A_{i-1}^{-1}\left(\hat{\alpha}_{1}\right)-A_{i-1}^{-1}\left(\hat{\alpha}_{2}\right)\right) \partial_{\alpha^{\ell}} A_{i-1}(\alpha)\right)\right]_{\ell}\right|_{\alpha=\alpha_{2}^{*}+u\left(\hat{\alpha}_{2}-\alpha_{2}^{*}\right)} \mathrm{d} u\left(\hat{\alpha}_{2}-\alpha_{2}^{*}\right) \\
& \quad+h\left|A_{i-1}^{-1}\left(\hat{\alpha}_{1}\right)-A_{i-1}^{-1}\left(\hat{\alpha}_{2}\right)\right|\left|Q_{i-1}\left(\theta^{*}\right)\right|+R_{i-1}\left(h^{2}, \theta\right)
\end{aligned}
$$

and

$$
\begin{aligned}
& \sup _{\tau \in D_{n, M}^{\alpha}} \frac{\left|\varrho_{n}^{\alpha}\left(\tau: \hat{\alpha}_{1}, \hat{\alpha}_{2}\right)\right|}{[n \tau]-\left[n \tau_{*}^{\alpha}\right]} \\
& \leq \frac{1}{\sqrt{n}} \sup _{\tau \in D_{n, M}^{\alpha}}\left|\frac{1}{[n \tau]-\left[n \tau_{*}^{\alpha}\right]} \sum_{i=\left[n \tau_{*}^{\alpha}\right]+1}^{[n \tau]} \int_{0}^{1}\left[\operatorname{tr}\left(\left(A_{i-1}^{-1}\left(\hat{\alpha}_{1}\right)-A_{i-1}^{-1}\left(\hat{\alpha}_{2}\right)\right) \partial_{\alpha^{\ell}} A_{i-1}(\alpha)\right)\right]_{\ell}\right|_{\alpha=\alpha_{2}^{*}+u\left(\hat{\alpha}_{2}-\alpha_{2}^{*}\right)} \mathrm{d} u \mid \\
& \quad \times \sqrt{n}\left|\hat{\alpha}_{2}-\alpha_{2}^{*}\right|
\end{aligned}
$$




$$
\begin{aligned}
& \quad+\sup _{\tau \in D_{n, M}^{\alpha}} \frac{h}{[n \tau]-\left[n \tau_{*}^{\alpha}\right]} \sum_{i=\left[n \tau_{*}^{\alpha}\right]+1}^{[n \tau]}\left|A_{i-1}^{-1}\left(\hat{\alpha}_{1}\right)-A_{i-1}^{-1}\left(\hat{\alpha}_{2}\right)\right|\left|Q_{i-1}\left(\theta^{*}\right)\right| \\
& \quad+\sup _{\tau \in D_{n, M}^{\alpha}} \frac{h^{2}}{[n \tau]-\left[n \tau_{*}^{\alpha}\right]} \sum_{i=\left[n \tau_{*}^{\alpha}\right]+1}^{[n \tau]} R_{i-1}(1, \theta) \\
& \leq \frac{1}{\sqrt{n}} \sup _{x, \alpha_{k}}\left|\left[\operatorname{tr}\left(\left(A^{-1}\left(x, \alpha_{1}\right)-A^{-1}\left(x, \alpha_{2}\right)\right) \partial_{\alpha^{\ell}} A\left(x, \alpha_{3}\right)\right)\right]_{\ell}\right| \sqrt{n}\left|\hat{\alpha}_{2}-\alpha_{2}^{*}\right| \\
& \quad+h \sup _{x, \alpha_{k}}\left|A^{-1}\left(x, \alpha_{1}\right)-A^{-1}\left(x, \alpha_{2}\right)\right| \sup _{x, \theta}|Q(x, \theta)|+\frac{h^{2}}{M} \sum_{i=\left[n \tau_{*}^{\alpha}\right]+1}^{n} R_{i-1}(1, \theta) \\
& =o_{p}(1) .
\end{aligned}
$$

Hence, we see

$$
\begin{aligned}
P_{3, n}^{\alpha} & \leq P\left(\sup _{\tau \in D_{n, M}^{\alpha}} \frac{\left|\varrho_{n}^{\alpha}\left(\tau: \hat{\alpha}_{1}, \hat{\alpha}_{2}\right)\right|}{[n \tau]-\left[n \tau_{*}^{\alpha}\right]} \geq \delta, \hat{\alpha}_{1} \in \mathcal{O}_{\alpha_{1}^{*}}, \hat{\alpha}_{2} \in \mathcal{O}_{\alpha_{2}^{*}}\right)+P\left(\hat{\alpha}_{1} \notin \mathcal{O}_{\alpha_{1}^{*}}\right)+P\left(\hat{\alpha}_{2} \notin \mathcal{O}_{\alpha_{2}^{*}}\right) \\
& \leq 3 \epsilon
\end{aligned}
$$

for large $n$.

[iv] From the evaluations in Steps [i]-[iii], we have

$$
\varlimsup_{n \rightarrow \infty} P\left(n\left(\hat{\tau}_{n}^{\alpha}-\tau_{*}^{\alpha}\right)>M\right) \leq \gamma_{\alpha}(M)+9 \epsilon
$$

for any $M \geq 1$ and $\epsilon>0$. Therefore

$$
\varlimsup_{M \rightarrow \infty} \varlimsup_{n \rightarrow \infty} P\left(n\left(\hat{\tau}_{n}^{\alpha}-\tau_{*}^{\alpha}\right)>M\right) \leq 9 \epsilon .
$$

Proof of Corollary 1. It is sufficient to show, for all $\epsilon_{1} \in[0,1)$ and $M>0$,

$$
\varlimsup_{n \rightarrow \infty} P\left(n^{\epsilon_{1}}\left(\hat{\tau}_{n}^{\alpha}-\tau_{*}^{\alpha}\right)>M\right)=0 .
$$

Let $D_{n, M}^{\alpha}=\left\{\tau \in[0,1] \mid n^{\epsilon_{1}}\left(\tau-\tau_{*}^{\alpha}\right)>M\right\}$. Suppose that there exists $\delta_{1} \in\left(0, \frac{1-\epsilon_{1}}{2}\right)$ such that $n h^{1 /\left(\epsilon_{1}+\delta_{1}\right)} \longrightarrow$ 0 . We have

$$
\begin{aligned}
P\left(n^{\epsilon_{1}}\left(\hat{\tau}_{n}-\tau_{*}^{\alpha}\right)>M\right) \leq & P\left(\sup _{\tau \in D_{n, M}^{\alpha}} \frac{\left|\mathcal{M}_{n}^{\alpha}\left(\tau: \hat{\alpha}_{1}, \hat{\alpha}_{2}\right)\right|}{n^{-\delta_{1}}\left([n \tau]-\left[n \tau_{*}^{\alpha}\right]\right)} \geq 1\right)+P\left(\inf _{\tau \in D_{n, M}^{\alpha}} \frac{\mathcal{A}_{n}^{\alpha}\left(\tau: \hat{\alpha}_{1}, \hat{\alpha}_{2}\right)}{n^{-\delta_{1}}\left([n \tau]-\left[n \tau_{*}^{\alpha}\right]\right)} \leq 2\right) \\
& +P\left(\sup _{\tau \in D_{n, M}^{\alpha}} \frac{\left|\varrho_{n}^{\alpha}\left(\tau: \hat{\alpha}_{1}, \hat{\alpha}_{2}\right)\right|}{n^{-\delta_{1}}\left([n \tau]-\left[n \tau_{*}^{\alpha}\right]\right)} \geq 1\right) \\
= & : P_{1, n}^{\alpha}+P_{2, n}^{\alpha}+P_{3, n}^{\alpha} .
\end{aligned}
$$

[i] Evaluation of $P_{1, n}^{\alpha}$. For large $n$, we have

$$
\begin{aligned}
& P_{1, n}^{\alpha}=P\left(\sup _{\tau \in D_{n, M}^{\alpha}} \frac{\left|\mathcal{M}_{n}^{\alpha}\left(\tau: \hat{\alpha}_{1}, \hat{\alpha}_{2}\right)\right|}{n^{-\delta_{1}}\left([n \tau]-\left[n \tau_{*}^{\alpha}\right]\right)} \geq 1\right) \\
& \leq P\left(\sup _{\tau \in D_{n, M}^{\alpha}} \frac{\left|\mathcal{M}_{n}^{\alpha}\left(\tau: \hat{\alpha}_{1}, \hat{\alpha}_{2}\right)\right|}{n^{-\delta_{1}}\left([n \tau]-\left[n \tau_{*}^{\alpha}\right]\right)} \geq 1, \hat{\alpha}_{1} \in \mathcal{O}_{\alpha_{1}^{*}}, \hat{\alpha}_{2} \in \mathcal{O}_{\alpha_{2}^{*}}\right)+P\left(\hat{\alpha}_{1} \notin \mathcal{O}_{\alpha_{1}^{*}}\right)+P\left(\hat{\alpha}_{2} \notin \mathcal{O}_{\alpha_{2}^{*}}\right) \\
& \leq P\left(\sup _{\tau \in D_{n, M}^{\alpha}} \frac{\sup _{\alpha_{k} \in \mathcal{O}_{\alpha_{k}^{*}}}\left|\mathcal{M}_{n}^{\alpha}\left(\tau: \alpha_{1}, \alpha_{2}\right)\right|}{[n \tau]-\left[n \tau_{*}^{\alpha}\right]} \geq n^{-\delta_{1}}\right)+P\left(\hat{\alpha}_{1} \notin \mathcal{O}_{\alpha_{1}^{*}}\right)+P\left(\hat{\alpha}_{2} \notin \mathcal{O}_{\alpha_{2}^{*}}\right) .
\end{aligned}
$$

By the uniform version on the Hájek-Renyi inequality in Lemma 2 of Iacus and Yoshida (2012), we obtain

$$
P\left(\sup _{\tau \in D_{n, M}^{\alpha}} \frac{1}{[n \tau]-\left[n \tau_{*}^{\alpha}\right]} \sup _{\alpha_{k} \in \mathcal{O}_{\alpha_{k}^{*}}}\left|\mathcal{M}_{n}^{\alpha}\left(\tau: \alpha_{1}, \alpha_{2}\right)\right| \geq n^{-\delta_{1}}\right)
$$




$$
\begin{aligned}
& \leq P\left(\max _{j>M / n^{\epsilon_{1}-1}-1} \frac{1}{j} \sup _{\alpha_{k} \in \mathcal{O}_{\alpha_{k}^{*}}}\left|\sum_{i=\left[n \tau_{*}^{\alpha}\right]+1}^{\left[n \tau_{*}^{\alpha}\right]+j}\left(F_{i}\left(\alpha_{1}\right)-F_{i}\left(\alpha_{2}\right)-\mathbb{E}\left[F_{i}\left(\alpha_{1}\right)-F_{i}\left(\alpha_{2}\right) \mid \mathscr{G}_{i-1}^{n}\right]\right)\right| \geq n^{-\delta_{1}}\right) \\
& \leq \sum_{j>M / n^{\epsilon_{1}-1}-1} \frac{C}{\left(n^{-\delta_{1}} j\right)^{2}} \\
& \leq \frac{C^{\prime}}{n^{-2 \delta_{1}}} \frac{n^{\epsilon_{1}-1}}{M}=\frac{C^{\prime}}{M} n^{\epsilon_{1}+2 \delta_{1}-1} \longrightarrow 0 .
\end{aligned}
$$

From [C6-I], we have $P\left(\hat{\alpha}_{k} \notin \mathcal{O}_{\alpha_{k}^{*}}\right)<\epsilon$ for large $n$. Therefore $P_{1, n}^{\alpha} \leq 3 \epsilon$ for large $n$.

[ii] Evaluation of $P_{2, n}^{\alpha}$. If $\hat{\alpha}_{k} \in \mathcal{O}_{\alpha_{k}^{*}}$, then there exists a positive constant $c$ independent of $i$ such that

$$
\begin{aligned}
\Gamma_{i-1}^{\alpha}\left(\hat{\alpha}_{1}, \hat{\alpha}_{2}\right) & =\Gamma_{i-1}^{\alpha}\left(\alpha_{1}^{*}, \alpha_{2}^{*}\right)+\left.\int_{0}^{1} \partial_{\left(\alpha_{1}, \alpha_{2}\right)} \Gamma_{i-1}^{\alpha}\left(\alpha_{1}, \alpha_{2}\right)\right|_{\alpha_{k}=\alpha_{k}^{*}+u\left(\hat{\alpha}_{k}-\alpha_{k}^{*}\right)} \mathrm{d} u\left(\begin{array}{c}
\hat{\alpha}_{1}-\alpha_{1}^{*} \\
\hat{\alpha}_{2}-\alpha_{2}^{*}
\end{array}\right) \\
& \geq \Gamma_{i-1}^{\alpha}\left(\alpha_{1}^{*}, \alpha_{2}^{*}\right)-c\left(\left|\hat{\alpha}_{1}-\alpha_{1}^{*}\right|+\left|\hat{\alpha}_{2}-\alpha_{2}^{*}\right|\right) .
\end{aligned}
$$

We see, for large $n$,

$$
\begin{aligned}
& P_{2, n}^{\alpha} \leq P\left(\inf _{\tau \in D_{n, M}^{\alpha}} \frac{\mathcal{A}_{n}^{\alpha}\left(\tau: \hat{\alpha}_{1}, \hat{\alpha}_{2}\right)}{n^{-\delta_{1}}\left([n \tau]-\left[n \tau_{*}^{\alpha}\right]\right)} \leq 2, \hat{\alpha}_{1} \in \mathcal{O}_{\alpha_{1}^{*}}, \hat{\alpha}_{2} \in \mathcal{O}_{\alpha_{2}^{*}}\right)+P\left(\hat{\alpha}_{1} \notin \mathcal{O}_{\alpha_{1}^{*}}\right)+P\left(\hat{\alpha}_{2} \notin \mathcal{O}_{\alpha_{2}^{*}}\right) \\
& \leq P\left(\inf _{\tau \in D_{n, M}^{\alpha}} \frac{1}{[n \tau]-\left[n \tau_{*}^{\alpha}\right]} \sum_{i=\left[n \tau_{*}^{\alpha}\right]+1}^{[n \tau]}\left(\Gamma_{i-1}^{\alpha}\left(\alpha_{1}^{*}, \alpha_{2}^{*}\right)-c\left(\left|\hat{\alpha}_{1}-\alpha_{1}^{*}\right|+\left|\hat{\alpha}_{2}-\alpha_{2}^{*}\right|\right)\right) \leq 2 n^{-\delta_{1}}\right) \\
& +P\left(\hat{\alpha}_{1} \notin \mathcal{O}_{\alpha_{1}^{*}}\right)+P\left(\hat{\alpha}_{2} \notin \mathcal{O}_{\alpha_{2}^{*}}\right) \\
& \leq P\left(\inf _{\tau \in D_{n, M}^{\alpha}} \frac{1}{[n \tau]-\left[n \tau_{*}^{\alpha}\right]} \sum_{i=\left[n \tau_{*}^{\alpha}\right]+1}^{[n \tau]} \Gamma_{i-1}^{\alpha}\left(\alpha_{1}^{*}, \alpha_{2}^{*}\right)-c\left(\left|\hat{\alpha}_{1}-\alpha_{1}^{*}\right|+\left|\hat{\alpha}_{2}-\alpha_{2}^{*}\right|\right) \leq 2 n^{-\delta_{1}}\right) \\
& +P\left(\hat{\alpha}_{1} \notin \mathcal{O}_{\alpha_{1}^{*}}\right)+P\left(\hat{\alpha}_{2} \notin \mathcal{O}_{\alpha_{2}^{*}}\right) \\
& \leq P\left(\inf _{\tau \in D_{n, M}^{\alpha}} \frac{1}{[n \tau]-\left[n \tau_{*}^{\alpha}\right]} \sum_{i=\left[n \tau_{*}^{\alpha}\right]+1}^{[n \tau]} \Gamma_{i-1}^{\alpha}\left(\alpha_{1}^{*}, \alpha_{2}^{*}\right) \leq 3 n^{-\delta_{1}}\right)+P\left(-c\left(\left|\hat{\alpha}_{1}-\alpha_{1}^{*}\right|+\left|\hat{\alpha}_{2}-\alpha_{2}^{*}\right|\right) \leq-n^{-\delta_{1}}\right) \\
& +P\left(\hat{\alpha}_{1} \notin \mathcal{O}_{\alpha_{1}^{*}}\right)+P\left(\hat{\alpha}_{2} \notin \mathcal{O}_{\alpha_{2}^{*}}\right) \\
& \leq P\left(\inf _{x} \Gamma^{\alpha}\left(x, \alpha_{1}^{*}, \alpha_{2}^{*}\right) \leq 3 n^{-\delta_{1}}\right)+P\left(\left|\hat{\alpha}_{1}-\alpha_{1}^{*}\right|+\left|\hat{\alpha}_{2}-\alpha_{2}^{*}\right| \geq \frac{n^{-\delta_{1}}}{c}\right)+P\left(\hat{\alpha}_{1} \notin \mathcal{O}_{\alpha_{1}^{*}}\right)+P\left(\hat{\alpha}_{2} \notin \mathcal{O}_{\alpha_{2}^{*}}\right) \\
& \leq 5 \epsilon
\end{aligned}
$$

thanks to

$$
P\left(\left|\hat{\alpha}_{1}-\alpha_{1}^{*}\right|+\left|\hat{\alpha}_{2}-\alpha_{2}^{*}\right| \geq \frac{n^{-\delta_{1}}}{c}\right) \leq P\left(n^{\delta_{1}-1 / 2} \sqrt{n}\left|\hat{\alpha}_{1}-\alpha_{1}^{*}\right| \geq \frac{1}{2 c}\right)+P\left(n^{\delta_{1}-1 / 2} \sqrt{n}\left|\hat{\alpha}_{2}-\alpha_{2}^{*}\right| \geq \frac{1}{2 c}\right) \leq 2 \epsilon
$$

from [C6-I].

[iii] Evaluation of $P_{3, n}^{\alpha}$.

$$
\begin{aligned}
& \operatorname{tr}\left(\left(A_{i-1}^{-1}\left(\hat{\alpha}_{1}\right)-A_{i-1}^{-1}\left(\hat{\alpha}_{2}\right)\right)\left(A_{i-1}\left(\hat{\alpha}_{2}\right)-h^{-1} \mathbb{E}_{\alpha_{2}^{*}}\left[\left(\Delta X_{i}\right)^{\otimes 2} \mid G_{i-1}^{n}\right]\right)\right) \\
& =\operatorname{tr}\left(\left(A_{i-1}^{-1}\left(\hat{\alpha}_{1}\right)-A_{i-1}^{-1}\left(\hat{\alpha}_{2}\right)\right)\left(A_{i-1}\left(\hat{\alpha}_{2}\right)-A_{i-1}\left(\alpha_{2}^{*}\right)\right)\right)+R_{i-1}(h, \theta) \\
& \leq\left.\int_{0}^{1}\left[\operatorname{tr}\left(\left(A_{i-1}^{-1}\left(\hat{\alpha}_{1}\right)-A_{i-1}^{-1}\left(\hat{\alpha}_{2}\right)\right) \partial_{\alpha^{\ell}} A_{i-1}(\alpha)\right)\right]_{\ell}\right|_{\alpha=\alpha_{2}^{*}+u\left(\hat{\alpha}_{2}-\alpha_{2}^{*}\right)} \mathrm{d} u\left(\hat{\alpha}_{2}-\alpha_{2}^{*}\right)+R_{i-1}(h, \theta)
\end{aligned}
$$

and

$$
\sup _{\tau \in D_{n, M}^{\alpha}} \frac{\left|\varrho_{n}^{\alpha}\left(\tau: \hat{\alpha}_{1}, \hat{\alpha}_{2}\right)\right|}{n^{-\delta_{1}}\left([n \tau]-\left[n \tau_{*}^{\alpha}\right]\right)}
$$




$$
\begin{aligned}
& \leq \frac{1}{n^{-\delta_{1}+1 / 2}} \sup _{\tau \in D_{n, M}^{\alpha}}\left|\frac{1}{[n \tau]-\left[n \tau_{*}^{\alpha}\right]} \sum_{i=\left[n \tau_{*}^{\alpha}\right]+1}^{[n \tau]} \int_{0}^{1}\left[\operatorname{tr}\left(\left(A_{i-1}^{-1}\left(\hat{\alpha}_{1}\right)-A_{i-1}^{-1}\left(\hat{\alpha}_{2}\right)\right) \partial_{\alpha^{\ell}} A_{i-1}(\alpha)\right)\right]_{\ell}\right|_{\alpha=\alpha_{2}^{*}+u\left(\hat{\alpha}_{2}-\alpha_{2}^{*}\right)} \mathrm{d} u \mid \\
& \quad \times \sqrt{n}\left|\hat{\alpha}_{2}-\alpha_{2}^{*}\right| \\
& \quad+\frac{h}{n^{-\delta_{1}}} \sup _{\tau \in D_{n, M}^{\alpha}} \frac{1}{[n \tau]-\left[n \tau_{*}^{\alpha}\right]} \sum_{i=\left[n \tau_{*}^{\alpha}\right]+1}^{[n \tau]} R_{i-1}(1, \theta) \\
& \leq \frac{1}{n^{-\delta_{1}+1 / 2}} \sup _{x, \alpha_{k}}\left|\left[\operatorname{tr}\left(\left(A^{-1}\left(x, \alpha_{1}\right)-A^{-1}\left(x, \alpha_{2}\right)\right) \partial_{\alpha^{\ell}} A\left(x, \alpha_{3}\right)\right)\right]_{\ell}\right| \sqrt{n}\left|\hat{\alpha}_{2}-\alpha_{2}^{*}\right| \\
& \quad+\frac{h}{n^{-\delta_{1}}} \frac{n^{\epsilon_{1}-1}}{M} \sum_{i=\left[n \tau_{*}^{\alpha}\right]+1}^{n} R_{i-1}(1, \theta) \\
& =O_{p}\left(n^{\delta_{1}-1 / 2}\right)+O_{p}\left(n^{\epsilon_{1}+\delta_{1}} h\right)=O_{p}\left(n^{\delta_{1}-1 / 2}\right)+O_{p}\left(n h^{1 /\left(\epsilon_{1}+\delta_{1}\right)}\right)=o_{p}(1) .
\end{aligned}
$$

Hence, we see

$$
\begin{aligned}
P_{3, n}^{\alpha} & \leq P\left(\sup _{\tau \in D_{n, M}^{\alpha}} \frac{\left|\varrho_{n}^{\alpha}\left(\tau: \hat{\alpha}_{1}, \hat{\alpha}_{2}\right)\right|}{n^{-\delta_{1}}\left([n \tau]-\left[n \tau_{*}^{\alpha}\right]\right)} \geq 1, \hat{\alpha}_{1} \in \mathcal{O}_{\alpha_{1}^{*}}, \hat{\alpha}_{2} \in \mathcal{O}_{\alpha_{2}^{*}}\right)+P\left(\hat{\alpha}_{1} \notin \mathcal{O}_{\alpha_{1}^{*}}\right)+P\left(\hat{\alpha}_{2} \notin \mathcal{O}_{\alpha_{2}^{*}}\right) \\
& \leq 3 \epsilon
\end{aligned}
$$

for large $n$.

[iv] From the evaluations in Steps [i]-[iii], we have

$$
\varlimsup_{n \rightarrow \infty} P\left(n^{\epsilon_{1}}\left(\hat{\tau}_{n}^{\alpha}-\tau_{*}^{\alpha}\right)>M\right) \leq 11 \epsilon
$$

for any $M>0$ and $\epsilon>0$.

In Case A of Situation II, we set

$$
\begin{aligned}
& \mathbb{G}_{n}(v)=\Psi_{n}\left(\tau_{*}^{\beta}+\frac{v}{T \vartheta_{\beta}^{2}}: \beta_{1}^{*}, \beta_{2}^{*} \mid \alpha^{*}\right)-\Psi_{n}\left(\tau_{*}^{\beta}: \beta_{1}^{*}, \beta_{2}^{*} \mid \alpha^{*}\right), \\
& \hat{\mathbb{G}}_{n}(v)=\Psi_{n}\left(\tau_{*}^{\beta}+\frac{v}{T \vartheta_{\beta}^{2}}: \hat{\beta}_{1}, \hat{\beta}_{2} \mid \hat{\alpha}\right)-\Psi_{n}\left(\tau_{*}^{\beta}: \hat{\beta}_{1}, \hat{\beta}_{2} \mid \hat{\alpha}\right), \\
& \mathcal{D}_{n}^{\beta}(v)=\hat{\mathbb{G}}_{n}(v)-\mathbb{G}_{n}(v) .
\end{aligned}
$$

Lemma 4 Suppose that [C1]-[C5], [C6-II], [A1-II]-[A3-II] hold. Then, for all $L>0$,

$$
\sup _{v \in[-L, L]}\left|\mathcal{D}_{n}^{\beta}(v)\right| \stackrel{p}{\longrightarrow} 0
$$

as $n \rightarrow \infty$.

Proof. We assume that $v>0$. Then, we can express

$$
\begin{aligned}
\mathcal{D}_{n}^{\beta}(v)= & \sum_{i=\left[n \tau_{*}^{\beta}\right]+1}^{\left[n \tau_{*}^{\beta}+v / h \vartheta_{\beta}^{2}\right]}\left(G_{i}\left(\hat{\beta}_{1} \mid \hat{\alpha}\right)-G_{i}\left(\hat{\beta}_{2} \mid \hat{\alpha}\right)\right)-\sum_{i=\left[n \tau_{*}^{\beta}\right]+1}^{\left[n \tau_{*}^{\beta}+v / h \vartheta_{\beta}^{2}\right]}\left(G_{i}\left(\beta_{1}^{*} \mid \alpha^{*}\right)-G_{i}\left(\beta_{2}^{*} \mid \alpha^{*}\right)\right) \\
= & \sum_{i=\left[n \tau_{*}^{\beta}\right]+1}^{\left[n \tau_{*}^{\beta}+v / h \vartheta_{\beta}^{2}\right]}\left(G_{i}\left(\hat{\beta}_{1} \mid \alpha^{*}\right)-G_{i}\left(\hat{\beta}_{2} \mid \alpha^{*}\right)+\left.\int_{0}^{1}\left(\partial_{\alpha} G_{i}\left(\hat{\beta}_{1} \mid \alpha\right)-\partial_{\alpha} G_{i}\left(\hat{\beta}_{2} \mid \alpha\right)\right)\right|_{\alpha=\alpha^{*}+u\left(\hat{\alpha}-\alpha^{*}\right)} \mathrm{d} u\left(\hat{\alpha}-\alpha^{*}\right)\right) \\
& -\sum_{i=\left[n \tau_{*}^{\beta}\right]+1}^{\left[n \tau_{*}^{\beta}+v / h \vartheta_{\beta}^{2}\right]}\left(G_{i}\left(\beta_{1}^{*} \mid \alpha^{*}\right)-G_{i}\left(\beta_{2}^{*} \mid \alpha^{*}\right)\right) \\
= & \sum_{i=\left[n \tau_{*}^{\beta}\right]+1}^{\left[n \tau_{*}^{\beta}+v / h \vartheta_{\beta}^{2}\right]}\left(G_{i}\left(\beta_{1}^{*} \mid \alpha^{*}\right)-G_{i}\left(\beta_{2}^{*} \mid \alpha^{*}\right)+\sum_{j=1}^{m-1} \frac{1}{j !}\left(\partial_{\beta}^{j} G_{i}\left(\beta_{1}^{*} \mid \alpha^{*}\right) \otimes\left(\hat{\beta}_{1}-\beta_{1}^{*}\right)^{\otimes j}-\partial_{\beta}^{j} G_{i}\left(\beta_{2}^{*} \mid \alpha^{*}\right) \otimes\left(\hat{\beta}_{2}-\beta_{2}^{*}\right)^{\otimes j}\right)\right.
\end{aligned}
$$




$$
\begin{aligned}
& +\int_{0}^{1} \frac{(1-u)^{m-1}}{(m-1) !} \partial_{\beta}^{m} G_{i}\left(\beta_{1}^{*}+u\left(\hat{\beta}_{1}-\beta_{1}^{*}\right) \mid \alpha^{*}\right) \mathrm{d} u \otimes\left(\hat{\beta}_{1}-\beta_{1}^{*}\right)^{\otimes m} \\
& -\int_{0}^{1} \frac{(1-u)^{m-1}}{(m-1) !} \partial_{\beta}^{m} G_{i}\left(\beta_{2}^{*}+u\left(\hat{\beta}_{2}-\beta_{2}^{*}\right) \mid \alpha^{*}\right) \mathrm{d} u \otimes\left(\hat{\beta}_{2}-\beta_{2}^{*}\right)^{\otimes m} \\
& \left.+\int_{0}^{1} \int_{0}^{1} \partial_{\beta} \partial_{\alpha} G_{i}\left(\hat{\beta}_{2}+v\left(\hat{\beta}_{1}-\hat{\beta}_{2}\right) \mid \alpha^{*}+u\left(\hat{\alpha}-\alpha^{*}\right)\right) \mathrm{d} u \mathrm{~d} v \otimes\left(\hat{\alpha}-\alpha^{*}\right) \otimes\left(\hat{\beta}_{1}-\hat{\beta}_{2}\right)\right) \\
= & \sum_{i=\left[n \tau_{*}^{\beta}\right]+1}^{\left[n \tau_{*}^{\beta}+v / h \vartheta_{\beta}^{2}\right]}\left(G_{i}\left(\beta_{1}^{*} \mid \alpha^{*}\right)-G_{i}\left(\beta_{2}^{*} \mid \alpha^{*}\right)\right) \\
\sum_{i=\left[n \tau_{*}^{\beta}\right]+1}^{\left.m+v / h \vartheta_{\beta}^{2}\right]} & \sum_{j=1}^{\left[n \tau_{*}^{\beta}+v / h \vartheta_{\beta}^{2}\right]}\left(\partial_{\beta}^{j} G_{i}\left(\beta_{1}^{*} \mid \alpha^{*}\right) \otimes\left(\hat{\beta}_{1}-\beta_{1}^{*}\right)^{\otimes j}-\partial_{\beta}^{j} G_{i}\left(\beta_{2}^{*} \mid \alpha^{*}\right) \otimes\left(\hat{\beta}_{2}-\beta_{2}^{*}\right)^{\otimes j}\right) \\
+ & \sum_{i=\left[n \tau_{*}^{\beta}\right]+1}^{1}\left(\int_{0}^{1} \frac{(1-u)^{m-1}}{(m-1) !} \partial_{\beta}^{m} G_{i}\left(\beta_{1}^{*}+u\left(\hat{\beta}_{1}-\beta_{1}^{*}\right) \mid \alpha^{*}\right) \mathrm{d} u \otimes\left(\hat{\beta}_{1}-\beta_{1}^{*}\right)^{\otimes m}\right. \\
& -\int_{0}^{1} \frac{(1-u)^{m-1}}{(m-1) !} \partial_{\beta}^{m} G_{i}\left(\beta_{2}^{*}+u\left(\hat{\beta}_{2}-\beta_{2}^{*}\right) \mid \alpha^{*}\right) \mathrm{d} u \otimes\left(\hat{\beta}_{2}-\beta_{2}^{*}\right)^{\otimes m} \\
& \left.+\int_{0}^{1} \int_{0}^{1} \partial_{\beta} \partial_{\alpha} G_{i}\left(\hat{\beta}_{2}+v\left(\hat{\beta}_{1}-\hat{\beta}_{2}\right) \mid \alpha^{*}+u\left(\hat{\alpha}-\alpha^{*}\right)\right) \mathrm{d} u \mathrm{~d} v \otimes\left(\hat{\alpha}-\alpha^{*}\right) \otimes\left(\hat{\beta}_{1}-\hat{\beta}_{2}\right)\right) .
\end{aligned}
$$

Now we see

$$
\begin{aligned}
& \sup _{v \in[0, L]}\left|\sum_{i=\left[n \tau_{*}^{\beta}\right]+1}^{\left[n \tau_{*}^{\beta}+v / h \vartheta_{\beta}^{2}\right]} \int_{0}^{1} \frac{(1-u)^{m-1}}{(m-1) !} \partial_{\beta}^{m} G_{i}\left(\beta_{j}^{*}+u\left(\hat{\beta}_{j}-\beta_{j}^{*}\right) \mid \alpha^{*}\right) \mathrm{d} u \otimes\left(\hat{\beta}_{k}-\beta_{k}^{*}\right)^{\otimes m}\right| \\
& \leq \sum_{i=\left[n \tau_{*}^{\beta}\right]+1}^{\left[n \tau_{*}^{\beta}+L / h \vartheta_{\beta}^{2}\right]}\left|\int_{0}^{1} \frac{(1-u)^{m-1}}{(m-1) !} \partial_{\beta}^{m} G_{i}\left(\beta_{k}^{*}+u\left(\hat{\beta}_{k}-\beta_{k}^{*}\right) \mid \alpha^{*}\right) \mathrm{d} u\right|\left|\hat{\beta}_{k}-\beta_{k}^{*}\right|^{m} \\
& \leq \frac{1}{T^{m / 2}} \sum_{i=\left[n \tau_{*}^{\beta}\right]+1}^{\left[n \tau_{*}^{\beta}+L / h \vartheta_{\beta}^{2}\right]} \sup _{\beta \in \Theta_{B}}\left|\partial_{\beta}^{m} G_{i}\left(\beta \mid \alpha^{*}\right)\right|\left(\sqrt{T}\left|\hat{\beta}_{k}-\beta_{k}^{*}\right|\right)^{m} \\
& =O_{p}\left(\frac{1}{T^{m / 2} h^{1 / 2} \vartheta_{\beta}^{2}}\right)=O_{p}\left(\frac{1}{n^{m / 2-1} h^{(m-1) / 2}} \frac{1}{T \vartheta_{\beta}^{2}}\right) \\
& =o_{p}(1) \text {, } \\
& \sup _{v \in[0, L]}\left|\sum_{i=\left[n \tau_{*}^{\beta}\right]+1}^{\left[n \tau_{*}^{\beta}+v / h \vartheta_{\beta}^{2}\right]} \int_{0}^{1} \int_{0}^{1} \partial_{\beta} \partial_{\alpha} G_{i}\left(\hat{\beta}_{2}+v\left(\hat{\beta}_{1}-\hat{\beta}_{2}\right) \mid \alpha^{*}+u\left(\hat{\alpha}-\alpha^{*}\right)\right) \mathrm{d} u \mathrm{~d} v \otimes\left(\hat{\alpha}-\alpha^{*}\right) \otimes\left(\hat{\beta}_{1}-\hat{\beta}_{2}\right)\right| \\
& \leq \sum_{i=\left[n \tau_{*}^{\beta}\right]+1}^{\left[n \tau_{*}^{\beta}+L / h \vartheta_{\beta}^{2}\right]}\left|\int_{0}^{1} \int_{0}^{1} \partial_{\beta} \partial_{\alpha} G_{i}\left(\hat{\beta}_{2}+v\left(\hat{\beta}_{1}-\hat{\beta}_{2}\right) \mid \alpha^{*}+u\left(\hat{\alpha}-\alpha^{*}\right)\right) \mathrm{d} u \mathrm{~d} v\right|\left|\hat{\alpha}-\alpha^{*}\right|\left|\hat{\beta}_{1}-\hat{\beta}_{2}\right| \\
& \leq \frac{\vartheta_{\beta}}{\sqrt{n}} \sum_{i=\left[n \tau_{*}^{\beta}\right]+1}^{\left[n \tau_{*}^{\beta}+L / h \vartheta_{\beta}^{2}\right]} \sup _{(\alpha, \beta) \in \Theta}\left|\partial_{\beta} \partial_{\alpha} G_{i}(\beta \mid \alpha)\right| \vartheta_{\beta}^{-1}\left|\hat{\beta}_{1}-\hat{\beta}_{2}\right| \sqrt{n}\left|\hat{\alpha}-\alpha^{*}\right| \\
& =O_{p}\left(\frac{1}{\sqrt{T} \vartheta_{\beta}}\right)=o_{p}(1)
\end{aligned}
$$


and

$$
\begin{aligned}
& \sum_{i=\left[n \tau_{*}^{\beta}\right]+1}^{\left[n \tau_{*}^{\beta}+v / h \vartheta_{\beta}^{2}\right]} \partial_{\beta}^{j} G_{i}\left(\beta_{k}^{*} \mid \alpha^{*}\right) \otimes\left(\hat{\beta}_{k}-\beta_{k}^{*}\right)^{\otimes j} \\
& =\sum_{\substack{\left.i n \tau_{*}^{\beta}+v / h \vartheta_{\beta}^{2}\right]\\
}}\left(\partial_{\beta}^{j} G_{i}\left(\beta_{k}^{*} \mid \alpha^{*}\right)-\mathbb{E}_{\beta_{2}^{*}}\left[\partial_{\beta}^{j} G_{i}\left(\beta_{k}^{*} \mid \alpha^{*}\right) \mid \mathscr{G}_{i-1}^{n}\right]\right) \otimes\left(\hat{\beta}_{k}-\beta_{k}^{*}\right)^{\otimes j} \\
& \quad+\sum_{i=\left[n \tau_{*}^{\beta}\right]+1}^{\left[n \tau_{*}^{\beta}+v / h \vartheta_{\beta}^{2}\right]} \mathbb{E}_{\beta_{2}^{*}}\left[\partial_{\beta}^{j} G_{i}\left(\beta_{k}^{*} \mid \alpha^{*}\right) \mid \mathscr{G}_{i-1}^{n}\right] \otimes\left(\hat{\beta}_{k}-\beta_{k}^{*}\right)^{\otimes j} .
\end{aligned}
$$

By Theorem 2.11 of Hall and Heyde (1980), we have

$$
\begin{aligned}
& \mathbb{E}_{\beta_{2}^{*}}\left[\frac{1}{T^{j}} \sup _{v \in[0, L]}\left|\sum_{i=\left[n \tau_{*}^{\beta}\right]+1}^{\left[n \tau_{*}^{\beta}+v / h \vartheta_{\beta}^{2}\right]}\left(\partial_{\beta}^{j} G_{i}\left(\beta_{k}^{*} \mid \alpha^{*}\right)-\mathbb{E}_{\beta_{2}^{*}}\left[\partial_{\beta}^{j} G_{i}\left(\beta_{k}^{*} \mid \alpha^{*}\right) \mid \mathscr{G}_{i-1}^{n}\right]\right)\right|^{2}\right] \\
& \leq \frac{C}{T^{j}} \sum_{i=\left[n \tau_{*}^{\beta}\right]+1}^{\left[n \tau_{*}^{\beta}+L / h \vartheta_{\beta}^{2}\right]} \mathbb{E}_{\beta_{2}^{*}}\left[\mathbb{E}_{\beta_{2}^{*}}\left[\left|\partial_{\beta}^{j} G_{i}\left(\beta_{k}^{*} \mid \alpha^{*}\right)-\mathbb{E}\left[\partial_{\beta}^{j} G_{i}\left(\beta_{k}^{*} \mid \alpha^{*}\right) \mid \mathscr{G}_{i-1}^{n}\right]\right|^{2} \mid \mathscr{G}_{i-1}^{n}\right]\right] \\
& \leq \frac{C^{\prime}}{T} \frac{h}{h \vartheta_{\beta}^{2}}=\frac{C^{\prime}}{T \vartheta_{\beta}^{2}} \longrightarrow 0
\end{aligned}
$$

and

$$
\frac{1}{T^{j / 2}} \sup _{v \in[0, L]}\left|\sum_{i=\left[n \tau_{*}^{\beta}\right]+1}^{\left[n \tau_{*}^{\beta}+v / h \vartheta_{\beta}^{2}\right]}\left(\partial_{\beta}^{j} G_{i}\left(\beta_{k}^{*} \mid \alpha^{*}\right)-\mathbb{E}_{\beta_{2}^{*}}\left[\partial_{\beta}^{j} G_{i}\left(\beta_{k}^{*} \mid \alpha^{*}\right) \mid \mathscr{G}_{i-1}^{n}\right]\right)\right|=o_{p}(1) .
$$

Moreover, we see

$$
\begin{aligned}
& \sup _{v \in[0, L]}\left|\sum_{i=\left[n \tau_{*}^{\beta}\right]+1}^{\left[n \tau_{*}^{\beta}+v / h \vartheta_{\beta}^{2}\right]} \mathbb{E}_{\beta_{2}^{*}}\left[\partial_{\beta} G_{i}\left(\beta_{k}^{*} \mid \alpha^{*}\right) \mid \mathscr{G}_{i-1}^{n}\right]\right|\left|\hat{\beta}_{k}-\beta_{k}^{*}\right| \\
& =\sup _{v \in[0, L]}\left|\sum_{i=\left[n \tau_{*}^{\beta}\right]+1}^{\left[n \tau_{*}^{\beta}+v / h \vartheta_{\beta}^{2}\right]}\left(-2 h\left[\partial_{\beta^{\ell_{1}}} b_{i-1}\left(\beta_{k}^{*}\right)^{\top} A_{i-1}^{-1}\left(\alpha^{*}\right)\left(b_{i-1}\left(\beta_{2}^{*}\right)-b_{i-1}\left(\beta_{k}^{*}\right)\right)\right]_{\ell_{1}}+R_{i-1}\left(h^{2}, \theta\right)\right)\right|\left|\hat{\beta}_{k}-\beta_{k}^{*}\right| \\
& =\sup _{v \in[0, L]} \mid \sum_{i=\left[n \tau_{*}^{\beta}\right]+1}^{\left[n \tau_{*}^{\beta}+v / h \vartheta_{\beta}^{2}\right]}\left(-2 h\left(\Xi_{i-1}^{\beta}\left(\alpha^{*}, \beta_{k}^{*}\right)\left(\beta_{2}^{*}-\beta_{k}^{*}\right)\right.\right. \\
& \left.\quad+\int_{0}^{1}(1-u)\left[\partial_{\beta^{\ell_{1}}} b_{i-1}\left(\beta_{k}^{*}\right)^{\top} A_{i-1}^{-1}\left(\alpha^{*}\right) \partial_{\beta^{\ell_{3}}} \partial_{\beta^{\ell_{2}}} b_{i-1}\left(\beta_{k}^{*}+u\left(\beta_{2}^{*}-\beta_{k}^{*}\right)\right)\right]_{\ell_{1}, \ell_{2}, \ell_{3}} \mathrm{~d} u \otimes\left(\beta_{2}^{*}-\beta_{k}^{*}\right)^{\otimes 2}\right) \\
& \left.\quad+R_{i-1}\left(h^{2}, \theta\right)\right)|| \hat{\beta}_{k}-\beta_{k}^{*} \mid \\
& =\sup _{v \in[0, L]} \sum_{i=\left[n \tau_{*}^{\beta}\right]+1}^{\left[n \tau_{*}^{\beta}+v / h \vartheta_{\beta}^{2}\right]}\left(-2 h\left(\Xi_{i-1}^{\beta}\left(\alpha^{*}, \beta_{0}\right)\left(\beta_{2}^{*}-\beta_{k}^{*}\right)\right.\right. \\
& \quad+\int_{0}^{1} \partial_{\beta} \Xi_{i-1}^{\beta}\left(\alpha^{*}, \beta_{0}+u\left(\beta_{k}^{*}-\beta_{0}\right)\right) \mathrm{d} u \otimes\left(\beta_{2}^{*}-\beta_{k}^{*}\right) \otimes\left(\beta_{k}^{*}-\beta_{0}\right) \\
& \left.\quad+\int_{0}^{1}(1-u)\left[\partial_{\beta^{\ell_{1}}} b_{i-1}\left(\beta_{k}^{*}\right)^{\top} A_{i-1}^{-1}\left(\alpha^{*}\right) \partial_{\beta_{3} \ell_{3}} \partial_{\beta^{\ell_{2}}} b_{i-1}\left(\beta_{k}^{*}+u\left(\beta_{2}^{*}-\beta_{k}^{*}\right)\right)\right]_{\ell_{1}, \ell_{2}, \ell_{3}} \mathrm{~d} u \otimes\left(\beta_{2}^{*}-\beta_{k}^{*}\right)^{\otimes 2}\right)
\end{aligned}
$$




$$
\begin{aligned}
& \left.\quad+R_{i-1}\left(h^{2}, \theta\right)\right)|| \hat{\beta}_{k}-\beta_{k}^{*} \mid \\
& \leq \frac{2 h \vartheta_{\beta}}{\sqrt{T}} \sup _{v \in[0, L]}\left|\sum_{i=\left[n \tau_{*}^{\beta}\right]+1}^{\left[n \tau_{*}^{\beta}+v / h \vartheta_{\beta}^{2}\right]} \Xi_{i-1}^{\beta}\left(\alpha^{*}, \beta_{0}\right)\right| \vartheta_{\beta}^{-1}\left|\beta_{2}^{*}-\beta_{k}^{*}\right| \sqrt{T}\left|\hat{\beta}_{k}-\beta_{k}^{*}\right| \\
& \quad+\frac{h \vartheta_{\beta}^{2}}{\sqrt{T}} \sum_{i=\left[n \tau_{*}^{\beta}\right]+1}^{\left[n \tau_{*}^{\beta}+L / h \vartheta_{\beta}^{2}\right]} R_{i-1}(1, \theta) \vartheta_{\beta}^{-1}\left|\beta_{2}^{*}-\beta_{k}^{*}\right| \vartheta_{\beta}^{-1}\left|\beta_{k}^{*}-\beta_{0}\right| \sqrt{T}\left|\hat{\beta}_{k}-\beta_{k}^{*}\right| \\
& \quad+\frac{h \vartheta_{\beta}^{2}}{\sqrt{T}} \sum_{i=\left[n \tau_{*}^{\beta}+L / h \vartheta_{\beta}^{2}\right]}^{[n]+1} R_{i-1}(1, \theta)\left(\vartheta_{\beta}^{-1}\left|\beta_{2}^{*}-\beta_{k}^{*}\right|\right)^{2} \sqrt{T}\left|\hat{\beta}_{k}-\beta_{k}^{*}\right|+\frac{1}{\sqrt{T}} \sum_{i=\left[n \tau_{*}^{\beta}\right]+1}^{\left[n \tau_{*}^{\beta}+L / h \vartheta_{\beta}^{2}\right]} R_{i-1}\left(h^{2}, \theta\right) \sqrt{T}\left|\hat{\beta}_{k}-\beta_{k}^{*}\right| \\
& =O_{p}\left(\frac{1}{\sqrt{T} \vartheta_{\beta}}\right)+O_{p}\left(\frac{1}{\sqrt{T}}\right)+O_{p}\left(\frac{h}{\sqrt{T} \vartheta_{\beta}^{2}}\right) \\
& =o_{p}(1)
\end{aligned}
$$

and, for $j \geq 2$,

$$
\begin{aligned}
\sup _{v \in[0, L]}\left|\sum_{i=\left[n \tau_{*}^{\beta}\right]+1}^{\left[n \tau_{*}^{\beta}+v / h \vartheta_{\beta}^{2}\right]} \mathbb{E}_{\beta_{2}^{*}}\left[\partial_{\beta}^{j} G_{i}\left(\beta_{k}^{*} \mid \alpha^{*}\right) \mid \mathscr{G}_{i-1}^{n}\right]\right|\left|\hat{\beta}_{k}-\beta_{k}^{*}\right|^{j} & \leq \sum_{i=\left[n \tau_{*}^{\beta}\right]+1}^{\left[n \tau_{*}^{\beta}+L / h \vartheta_{\beta}^{2}\right]} R_{i-1}(h, \theta)\left|\hat{\beta}_{j}-\beta_{j}^{*}\right|^{j} \\
& =\frac{h}{T^{j / 2}} \sum_{i=\left[n \tau_{*}^{\beta}\right]+1}^{\left[n \tau_{*}^{\beta}+L / h \vartheta_{\beta}^{2}\right]} R_{i-1}(1, \theta)\left(\sqrt{T}\left|\hat{\beta}_{k}-\beta_{k}^{*}\right|\right)^{j} \\
& =O_{p}\left(\frac{1}{T \vartheta_{\beta}^{2}}\right) \\
& =o_{p}(1) .
\end{aligned}
$$

Therefore, from (7.29)-7.35), we have

$$
\sup _{v \in[0, L]}\left|\mathcal{D}_{n}^{\beta}(v)\right| \stackrel{p}{\longrightarrow} 0 .
$$

By the similar proof, we see $\sup _{v \in[-L, 0]}\left|\mathcal{D}_{n}^{\beta}(v)\right| \stackrel{p}{\longrightarrow} 0$, and this proof is complete.

Lemma 5 Suppose that [C1]-[C5], [C6-II], [A1-II]-[A3-II] hold. Then, for all $L>0$,

$$
\mathbb{G}_{n}(v) \stackrel{w}{\longrightarrow} \mathbb{G}(v) \text { in } \mathbb{D}[-L, L]
$$

as $n \rightarrow \infty$.

Proof. We consider $v>0$. We have

$$
\begin{aligned}
\mathbb{G}_{n}(v)= & \sum_{i=\left[n \tau_{*}^{\beta}\right]+1}^{\left[n \tau_{*}^{\beta}+v / h \vartheta_{\beta}^{2}\right]}\left(G_{i}\left(\beta_{1}^{*} \mid \alpha^{*}\right)-G_{i}\left(\beta_{2}^{*} \mid \alpha^{*}\right)\right) \\
= & \sum_{i=\left[n \tau_{*}^{\beta}\right]+1}^{\left[n \tau_{*}^{\beta}+v / h \vartheta_{\beta}^{2}\right]}\left(\sum_{j=1}^{m-1} \frac{1}{j !} \partial_{\beta}^{j} G_{i}\left(\beta_{2}^{*} \mid \alpha^{*}\right) \otimes\left(\beta_{1}^{*}-\beta_{2}^{*}\right)^{\otimes j}\right. \\
& \left.\quad+\int_{0}^{1} \frac{(1-u)^{m-1}}{(m-1) !} \partial_{\beta}^{m} G_{i}\left(\beta_{2}^{*}+u\left(\beta_{1}^{*}-\beta_{2}^{*}\right) \mid \alpha^{*}\right) \mathrm{d} u \otimes\left(\beta_{1}^{*}-\beta_{2}^{*}\right)^{\otimes m}\right) \\
= & \sum_{i=\left[n \tau_{*}^{\beta}\right]+1}^{\left[n \tau_{*}^{\beta}+v / h \vartheta_{\beta}^{2}\right]}\left(\partial_{\beta} G_{i}\left(\beta_{2}^{*} \mid \alpha^{*}\right)\left(\beta_{1}^{*}-\beta_{2}^{*}\right)+\frac{1}{2} \partial_{\beta}^{2} G_{i}\left(\beta_{2}^{*} \mid \alpha^{*}\right) \otimes\left(\beta_{1}^{*}-\beta_{2}^{*}\right)^{\otimes 2}\right)
\end{aligned}
$$




$$
\begin{aligned}
& +\sum_{i=\left[n \tau_{*}^{\beta}\right]+1}^{\left[n \tau_{*}^{\beta}+v / h \vartheta_{\beta}^{2}\right]}\left(\sum_{j=3}^{m-1} \frac{1}{j !} \partial_{\beta}^{j} G_{i}\left(\beta_{2}^{*} \mid \alpha^{*}\right) \otimes\left(\beta_{1}^{*}-\beta_{2}^{*}\right)^{\otimes j}\right. \\
& \left.\quad+\int_{0}^{1} \frac{(1-u)^{m-1}}{(m-1) !} \partial_{\beta}^{m} G_{i}\left(\beta_{2}^{*}+u\left(\beta_{1}^{*}-\beta_{2}^{*}\right) \mid \alpha^{*}\right) \mathrm{d} u \otimes\left(\beta_{1}^{*}-\beta_{2}^{*}\right)^{\otimes m}\right) \\
& =\sum_{i=\left[n \tau_{*}^{\beta}\right]+1}^{\left[n \tau_{*}^{\beta}+v / h \vartheta_{\beta}^{2}\right]}\left(\partial_{\beta} G_{i}\left(\beta_{2}^{*} \mid \alpha^{*}\right)\left(\beta_{1}^{*}-\beta_{2}^{*}\right)+\frac{1}{2} \partial_{\beta}^{2} G_{i}\left(\beta_{2}^{*} \mid \alpha^{*}\right) \otimes\left(\beta_{1}^{*}-\beta_{2}^{*}\right)^{\otimes 2}\right)+\bar{o}_{p}(1) \\
& =: \mathbb{G}_{1, n}(v)+\mathbb{G}_{2, n}(v)+\bar{o}_{p}(1),
\end{aligned}
$$

where, for $j \geq 3$,

$$
\begin{aligned}
& \vartheta_{\beta}^{j} \sup _{v \in[0, L]}\left|\sum_{i=\left[n \tau_{*}^{\beta}\right]+1}^{\left[n \tau_{*}^{\beta}+v / h \vartheta_{\beta}^{2}\right]}\left(\partial_{\beta}^{j} G_{i}\left(\beta_{2}^{*} \mid \alpha^{*}\right)-\mathbb{E}\left[\partial_{\beta}^{j} G_{i}\left(\beta_{2}^{*} \mid \alpha^{*}\right) \mid \mathscr{G}_{i-1}^{n}\right]\right)\right|=o_{p}(1), \\
& \vartheta_{\beta}^{j} \sup _{v \in[0, L]}\left|\sum_{i=\left[n \tau_{*}^{\beta}\right]+1}^{\left[n \tau_{*}^{\beta}+v / h \vartheta_{\beta}^{2}\right]} \mathbb{E}_{\beta_{2}^{*}}\left[\partial_{\beta}^{j} G_{i}\left(\beta_{2}^{*} \mid \alpha^{*}\right) \mid \mathscr{G}_{i-1}^{n}\right]\right|=o_{p}(1), \\
& \sup _{v \in[0, L]}\left|\sum_{i=\left[n \tau_{*}^{\beta}\right]+1}^{\left[n \tau_{*}^{\beta}+v / h \vartheta_{\beta}^{2}\right]} \int_{0}^{1} \frac{(1-u)^{m-1}}{(m-1) !} \partial_{\beta}^{m} G_{i}\left(\beta_{2}^{*}+u\left(\beta_{1}^{*}-\beta_{2}^{*}\right) \mid \alpha^{*}\right) \mathrm{d} u \otimes\left(\beta_{1}^{*}-\beta_{2}^{*}\right)^{\otimes m}\right|=o_{p}(1) .
\end{aligned}
$$

Now, we see

$$
\begin{aligned}
& \mathbb{G}_{1, n}(v)=\sum_{i=\left[n \tau_{*}^{\beta}\right]+1}^{\left[n \tau_{*}^{\beta}+v / h \vartheta_{\beta}^{2}\right]}\left(\partial_{\beta} G_{i}\left(\beta_{2}^{*} \mid \alpha^{*}\right)-\mathbb{E}_{\beta_{2}^{*}}\left[\partial_{\beta} G_{i}\left(\beta_{2}^{*} \mid \alpha^{*}\right) \mid \mathscr{G}_{i-1}^{n}\right]\right)\left(\beta_{1}^{*}-\beta_{2}^{*}\right) \\
& +\sum_{i=\left[n \tau_{*}^{\beta}\right]+1}^{\left[n \tau_{*}^{\beta}+v / h \vartheta_{\beta}^{2}\right]} \mathbb{E}_{\beta_{2}^{*}}\left[\partial_{\beta} G_{i}\left(\beta_{2}^{*} \mid \alpha^{*}\right) \mid \mathscr{G}_{i-1}^{n}\right]\left(\beta_{1}^{*}-\beta_{2}^{*}\right), \\
& \sup _{v \in[0, L]}\left|\sum_{i=\left[n \tau_{*}^{\beta}\right]+1}^{\left[n \tau_{*}^{\beta}+v / h \vartheta_{\beta}^{2}\right]} \mathbb{E}_{\beta_{2}^{*}}\left[\partial_{\beta} G_{i}\left(\beta_{2}^{*} \mid \alpha^{*}\right) \mid \mathscr{G}_{i-1}^{n}\right]\left(\beta_{1}^{*}-\beta_{2}^{*}\right)\right| \leq \vartheta_{\beta} \sum_{i=\left[n \tau_{*}^{\beta}\right]+1}^{\left[n \tau_{*}^{\beta}+L / h \vartheta_{\beta}^{2}\right]} R_{i-1}\left(h^{2}, \theta\right)=O_{p}\left(\frac{h}{\vartheta_{\beta}}\right)=o_{p}(1), \\
& \sum_{i=\left[n \tau_{*}^{\beta}\right]+1}^{\left[n \tau_{*}^{\beta}+v / h \vartheta_{\beta}^{2}\right]} \mathbb{E}_{\beta_{2}^{*}}\left[\left(\left(\partial_{\beta} G_{i}\left(\beta_{2}^{*} \mid \alpha^{*}\right)-\mathbb{E}_{\beta_{2}^{*}}\left[\partial_{\beta} G_{i}\left(\beta_{2}^{*} \mid \alpha^{*}\right) \mid \mathscr{G}_{i-1}^{n}\right]\right)\left(\beta_{1}^{*}-\beta_{2}^{*}\right)\right)^{2} \mid \mathscr{G}_{i-1}^{n}\right] \\
& =\left(\beta_{1}^{*}-\beta_{2}^{*}\right)^{\top} \sum_{i=\left[n \tau_{*}^{\beta}\right]+1}^{\left[n \tau_{*}^{\beta}+v / h \vartheta_{\beta}^{2}\right]} \mathbb{E}_{\beta_{2}^{*}}\left[\left(\partial_{\beta} G_{i}\left(\beta_{2}^{*} \mid \alpha^{*}\right)-\mathbb{E}_{\beta_{2}^{*}}\left[\partial_{\beta} G_{i}\left(\beta_{2}^{*} \mid \alpha^{*}\right) \mid \mathscr{G}_{i-1}^{n}\right]\right)^{\top}\right. \\
& \left.\left(\partial_{\beta} G_{i}\left(\beta_{2}^{*} \mid \alpha^{*}\right)-\mathbb{E}_{\beta_{2}^{*}}\left[\partial_{\beta} G_{i}\left(\beta_{2}^{*} \mid \alpha^{*}\right) \mid \mathscr{G}_{i-1}^{n}\right]\right) \mid \mathscr{G}_{i-1}^{n}\right]\left(\beta_{1}^{*}-\beta_{2}^{*}\right) \\
& =\left(\beta_{1}^{*}-\beta_{2}^{*}\right)^{\top} \sum_{i=\left[n \tau_{*}^{\beta}\right]+1}^{\left[n \tau_{*}^{\beta}+v / h \vartheta_{\beta}^{2}\right]}\left(4 h \Xi_{i-1}^{\beta}\left(\alpha^{*}, \beta_{2}^{*}\right)+R_{i-1}\left(h^{2}, \theta\right)\right)\left(\beta_{1}^{*}-\beta_{2}^{*}\right) \\
& \stackrel{p}{\longrightarrow} 4 e_{\beta}^{\top} \int_{\mathbb{R}^{d}} \Xi^{\beta}\left(x, \alpha^{*}, \beta_{0}\right) \mathrm{d} \mu_{\left(\alpha^{*}, \beta_{0}\right)}(x) e_{\beta} v=4 \mathcal{J}_{\beta} v
\end{aligned}
$$


and

$$
\begin{aligned}
& \sum_{i=\left[n \tau_{*}^{\beta}\right]+1}^{\left[n \tau_{*}^{\beta}+v / h \vartheta_{\beta}^{2}\right]} \mathbb{E}\left[\left(\left(\partial_{\beta} G_{i}\left(\beta_{2}^{*} \mid \alpha^{*}\right)-\mathbb{E}_{\beta_{2}^{*}}\left[\partial_{\beta} G_{i}\left(\beta_{2}^{*} \mid \alpha^{*}\right) \mid \mathscr{G}_{i-1}^{n}\right]\right)\left(\beta_{1}^{*}-\beta_{2}^{*}\right)\right)^{4} \mid \mathscr{G}_{i-1}^{n}\right] \\
= & \sum_{i=\left[n \tau_{*}^{\beta}\right]+1}^{\left[n \tau_{*}^{\beta}+v / h \vartheta_{\beta}^{2}\right]} \vartheta_{\beta}^{4} R_{i-1}\left(h^{2}, \theta\right) \stackrel{p}{\longrightarrow} 0 .
\end{aligned}
$$

According to Corollary 3.8 of McLeish (1974), we obtain, from (7.38) and 7.39),

$$
\sum_{i=\left[n \tau_{*}^{\beta}\right]+1}^{\left[n \tau_{*}^{\beta}+v / h \vartheta_{\beta}^{2}\right]}\left(\partial_{\beta} G_{i}\left(\beta_{2}^{*} \mid \alpha^{*}\right)-\mathbb{E}_{\beta_{2}^{*}}\left[\partial_{\beta} G_{i}\left(\beta_{2}^{*} \mid \alpha^{*}\right) \mid \mathscr{G}_{i-1}^{n}\right]\right)\left(\beta_{1}^{*}-\beta_{2}^{*}\right) \stackrel{w}{\longrightarrow}-2 \mathcal{J}_{\beta}^{1 / 2} \mathcal{W}(v) \text { in } \mathbb{D}[0, L] .
$$

Further, from (7.36), (7.37) and $(7.40)$, we have $\mathbb{G}_{1, n}(v) \stackrel{w}{\longrightarrow}-2 \mathcal{J}_{\beta}^{1 / 2} \mathcal{W}(v)$ in $\mathbb{D}[0, L]$.

Besides, we see

$$
\begin{aligned}
& \sup _{v \in[0, L]}\left|\sum_{i=\left[n \tau_{*}^{\beta}\right]+1}^{\left[n \tau_{*}^{\beta}+v / h \vartheta_{\beta}^{2}\right]} \partial_{\beta}^{2} G_{i}\left(\beta_{2}^{*} \mid \alpha^{*}\right) \otimes\left(\beta_{1}^{*}-\beta_{2}^{*}\right)^{\otimes 2}-2 \mathcal{J}_{\beta} v\right| \\
& \leq \sup _{v \in[0, L]}\left|\sum_{i=\left[n \tau_{*}^{\beta}\right]+1}^{\left[n \tau_{*}^{\beta}+v / h \vartheta_{\beta}^{2}\right]}\left(\partial_{\beta}^{2} G_{i}\left(\beta_{2}^{*} \mid \alpha^{*}\right)-\mathbb{E}_{\beta_{2}^{*}}\left[\partial_{\beta}^{2} G_{i}\left(\beta_{2}^{*} \mid \alpha^{*}\right) \mid \mathscr{G}_{i-1}^{n}\right]\right) \otimes\left(\beta_{1}^{*}-\beta_{2}^{*}\right)^{\otimes 2}\right| \\
& +\sup _{v \in[0, L]} \sum_{i=\left[n \tau_{*}^{\beta}\right]+1}^{\left[n \tau_{*}^{\beta}+v / h \vartheta_{\beta}^{2}\right]} \mathbb{E}_{\beta_{2}^{*}}\left[\partial_{\beta}^{2} G_{i}\left(\beta_{2}^{*} \mid \alpha^{*}\right) \mid \mathscr{G}_{i-1}^{n}\right] \otimes\left(\beta_{1}^{*}-\beta_{2}^{*}\right)^{\otimes 2}-2 \mathcal{J}_{\beta} v \mid, \\
& \sup _{v \in[0, L]}\left|\sum_{i=\left[n \tau_{*}^{\beta}\right]+1}^{\left[n \tau_{*}^{\beta}+v / h \vartheta_{\beta}^{2}\right]}\left(\partial_{\beta}^{2} G_{i}\left(\beta_{2}^{*} \mid \alpha^{*}\right)-\mathbb{E}_{\beta_{2}^{*}}\left[\partial_{\beta}^{2} G_{i}\left(\beta_{2}^{*} \mid \alpha^{*}\right) \mid \mathscr{G}_{i-1}^{n}\right]\right) \otimes\left(\beta_{1}^{*}-\beta_{2}^{*}\right)^{\otimes 2}\right| \stackrel{p}{\longrightarrow} 0
\end{aligned}
$$

and

$$
\begin{aligned}
& \sup _{v \in[0, L]}\left|\sum_{i=\left[n \tau_{*}^{\beta}\right]+1}^{\left[n \tau_{*}^{\beta}+v / h \vartheta_{\beta}^{2}\right]} \mathbb{E}_{\beta_{2}^{*}}\left[\partial_{\beta}^{2} G_{i}\left(\beta_{2}^{*} \mid \alpha^{*}\right) \mid \mathscr{G}_{i-1}^{n}\right] \otimes\left(\beta_{1}^{*}-\beta_{2}^{*}\right)^{\otimes 2}-2 \mathcal{J}_{\beta} v\right| \\
& =\sup _{v \in[0, L]}\left|\sum_{i=\left[n \tau_{*}^{\beta}\right]+1}^{\left[n \tau_{*}^{\beta}+v / h \vartheta_{\beta}^{2}\right]}\left(2 h \Xi_{i-1}^{\beta}\left(\alpha^{*}, \beta_{2}^{*}\right)+R_{i-1}\left(h^{2}, \theta\right)\right) \otimes\left(\beta_{1}^{*}-\beta_{2}^{*}\right)^{\otimes 2}-2 \mathcal{J}_{\beta} v\right| \\
& \leq 2 \sup _{v \in[0, L]}\left|\sum_{i=\left[n \tau_{*}^{\beta}\right]+1}^{\left[n \tau_{*}^{\beta}+v / h \vartheta_{\beta}^{2}\right]} h \Xi_{i-1}^{\beta}\left(\alpha^{*}, \beta_{0}\right) \otimes\left(\beta_{1}^{*}-\beta_{2}^{*}\right)^{\otimes 2}-\mathcal{J}_{\beta} v\right|+o_{p}(1) \\
& \stackrel{p}{\longrightarrow} 0,
\end{aligned}
$$

where 7.42 is obtained by

$$
\begin{aligned}
& \mathbb{E}_{\beta_{2}^{*}}\left[\sup _{v \in[0, L]}\left|\sum_{i=\left[n \tau_{*}^{\beta}\right]+1}^{\left[n \tau_{*}^{\beta}+v / h \vartheta_{\beta}^{2}\right]}\left(\partial_{\beta}^{2} G_{i}\left(\beta_{2}^{*} \mid \alpha^{*}\right)-\mathbb{E}_{\beta_{2}^{*}}\left[\partial_{\beta}^{2} G_{i}\left(\beta_{2}^{*} \mid \alpha^{*}\right) \mid \mathscr{G}_{i-1}^{n}\right]\right) \otimes\left(\beta_{1}^{*}-\beta_{2}^{*}\right)^{\otimes 2}\right|^{2}\right] \\
& \leq C \vartheta_{\beta}^{4} \sum_{i=\left[n \tau_{*}^{\beta}\right]+1}^{\left[n \tau_{*}^{\beta}+L / h \vartheta_{\beta}^{2}\right]} \mathbb{E}_{\beta_{2}^{*}}\left[\left|\partial_{\beta}^{2} G_{i}\left(\beta_{2}^{*} \mid \alpha^{*}\right)-\mathbb{E}_{\beta_{2}^{*}}\left[\partial_{\beta}^{2} G_{i}\left(\beta_{2}^{*} \mid \alpha^{*}\right) \mid \mathscr{G}_{i-1}^{n}\right]\right|^{2}\right] \\
& \leq C^{\prime} \vartheta_{\beta}^{2} \longrightarrow 0
\end{aligned}
$$


from Theorem 2.11 of Hall and Heyde (1980), and 7.43 is obtained by

$$
\begin{aligned}
& \sup _{v \in[0, L]}\left|\sum_{i=\left[n \tau_{*}^{\beta}\right]+1}^{\left[n \tau_{*}^{\beta}+v / h \vartheta_{\beta}^{2}\right]} h \Xi_{i-1}^{\beta}\left(\alpha^{*}, \beta_{0}\right) \otimes\left(\beta_{1}^{*}-\beta_{2}^{*}\right)^{\otimes 2}-\mathcal{J}_{\beta} v\right| \\
& \leq \sup _{v \in\left[0, \epsilon_{n}\right]}\left|\sum_{i=\left[n \tau_{*}^{\beta}\right]+1}^{\left[n \tau_{*}^{\beta}+v / h \vartheta_{\beta}^{2}\right]} h \Xi_{i-1}^{\beta}\left(\alpha^{*}, \beta_{0}\right) \otimes\left(\beta_{1}^{*}-\beta_{2}^{*}\right)^{\otimes 2}-\mathcal{J}_{\beta} v\right| \\
& \quad+\sup _{v \in\left[\epsilon_{n}, L\right]}\left|\sum_{i=\left[n \tau_{*}^{\beta}\right]+1}^{\left[n \tau_{*}^{\beta}+v / h \vartheta_{\beta}^{2}\right]} h \Xi_{i-1}^{\beta}\left(\alpha^{*}, \beta_{0}\right) \otimes\left(\beta_{1}^{*}-\beta_{2}^{*}\right)^{\otimes 2}-\mathcal{J}_{\beta} v\right| \\
& \leq \epsilon_{n} \sup _{v \in\left[0, \epsilon_{n}\right]}\left|\frac{1}{v} \sum_{i=\left[n \tau_{*}^{\beta}\right]+1}^{\left[n \tau_{*}^{\beta}+v / h \vartheta_{\beta}^{2}\right]} h \Xi_{i-1}^{\beta}\left(\alpha^{*}, \beta_{0}\right) \otimes\left(\beta_{1}^{*}-\beta_{2}^{*}\right)^{\otimes 2}-\mathcal{J}_{\beta}\right| \\
& \quad+L \sup _{v \in\left[\epsilon_{n}, L\right]} \sum_{i=\left[n \tau_{*}^{\beta}\right]+1}^{\left[\frac{1}{v} \tau_{*}^{\beta}+v / h \vartheta_{\beta}^{2}\right]} \sum_{i-1}^{\beta}\left(\alpha^{*}, \beta_{0}\right) \otimes\left(\beta_{1}^{*}-\beta_{2}^{*}\right)^{\otimes 2}-\mathcal{J}_{\beta} \mid \\
& \leq O_{p}\left(\epsilon_{n}\right)+L \max _{\left[n^{1 / r}\right] \leq k \leq n}\left|\frac{1}{k} \sum_{i=\left[n \tau_{*}^{\beta}\right]+1}^{\left[n \tau_{*}^{\beta}\right]+k} h \Xi_{i-1}^{\beta}\left(\alpha^{*}, \beta_{0}\right) \otimes\left(\vartheta_{\beta}^{-1}\left(\beta_{1}^{*}-\beta_{2}^{*}\right)\right)^{\otimes 2}-\mathcal{J}_{\beta}\right| \\
& =o_{p}(1)
\end{aligned}
$$

Here $\left\{\epsilon_{n}\right\}_{n=1}^{\infty}$ is a positive sequence such that $\epsilon_{n} \longrightarrow 0$ and $\epsilon_{n} / \vartheta_{\beta}^{2} \longrightarrow \infty$, and $r$ is a constant with $r \in(1,2)$ and $n h^{r} \longrightarrow \infty$. From (7.41)-(7.43), we have $\sup _{v \in[0, L]}\left|\mathbb{G}_{2, n}(v)-\mathcal{J}_{\beta} v\right| \stackrel{p}{\longrightarrow} 0$. Therefore we obtain

$$
\mathbb{G}_{n}(v) \stackrel{w}{\longrightarrow}-2 \mathcal{J}_{\beta}^{1 / 2} \mathcal{W}(v)+\mathcal{J}_{\beta} v \text { in } \mathbb{D}[0, L] .
$$

The argument for $v<0$ is proved as well.

Proof of Theorem 3, It is enough to show

$$
\varlimsup_{M \rightarrow \infty} \varlimsup_{n \rightarrow \infty} P\left(T \vartheta_{\beta}^{2}\left(\hat{\tau}_{n}^{\beta}-\tau_{*}^{\beta}\right)>M\right)=0 .
$$

Because in the same way as the proof of Theorem 1, (7.44) leads to $T \vartheta_{\beta}^{2}\left(\hat{\tau}_{n}^{\beta}-\tau_{*}^{\beta}\right)=O_{p}(1)$, and this and Lemmas 1, 4, 5yield

$$
T \vartheta_{\beta}^{2}\left(\hat{\tau}_{n}^{\beta}-\tau_{*}^{\beta}\right) \stackrel{d}{\longrightarrow} \arg \min _{v \in \mathbb{R}} \mathbb{G}(v) .
$$

We show (7.44) below. For $\tau>\tau_{*}^{\beta}$, we have

$$
\begin{aligned}
\Psi_{n}\left(\tau: \beta_{1}, \beta_{2} \mid \alpha\right)-\Psi_{n}\left(\tau_{*}^{\beta}: \beta_{1}, \beta_{2} \mid \alpha\right) & =\sum_{i=1}^{[n \tau]} G_{i}\left(\beta_{1} \mid \alpha\right)+\sum_{i=[n \tau]+1}^{n} G_{i}\left(\beta_{2} \mid \alpha\right)-\sum_{i=1}^{\left[n \tau_{*}^{\beta}\right]} G_{i}\left(\beta_{1} \mid \alpha\right)-\sum_{i=\left[n \tau_{*}^{\beta}\right]+1}^{n} G_{i}\left(\beta_{2} \mid \alpha\right) \\
& =\sum_{i=\left[n \tau_{*}^{\beta}\right]+1}^{[n \tau]}\left(G_{i}\left(\beta_{1} \mid \alpha\right)-G_{i}\left(\beta_{2} \mid \alpha\right)\right) .
\end{aligned}
$$

Now, from

$$
\begin{aligned}
G_{i}\left(\beta_{1} \mid \alpha\right)-G_{i}\left(\beta_{2} \mid \alpha\right)= & G_{i}\left(\beta_{1} \mid \alpha\right)-G_{i}\left(\beta_{2} \mid \alpha\right)-\mathbb{E}_{\beta_{2}^{*}}\left[G_{i}\left(\beta_{1} \mid \alpha\right)-G_{i}\left(\beta_{2} \mid \alpha\right) \mid \mathscr{G}_{i-1}^{n}\right] \\
& +h \operatorname{tr}\left(A_{i-1}^{-1}(\alpha)\left(b_{i-1}\left(\beta_{1}\right)-b_{i-1}\left(\beta_{2}\right)\right)^{\otimes 2}\right) \\
& +2 \operatorname{tr}\left(A_{i-1}^{-1}(\alpha)\left(h b_{i-1}\left(\beta_{2}\right)-\mathbb{E}_{\beta_{2}^{*}}\left[\Delta X_{i} \mid \mathscr{G}_{i-1}^{n}\right]\right)\left(b_{i-1}\left(\beta_{1}\right)-b_{i-1}\left(\beta_{2}\right)\right)^{\top}\right),
\end{aligned}
$$

we see

$$
\Psi_{n}\left(\tau: \beta_{1}, \beta_{2} \mid \alpha\right)-\Psi_{n}\left(\tau_{*}^{\beta}: \beta_{1}, \beta_{2} \mid \alpha\right)
$$




$$
\begin{aligned}
= & \sum_{i=\left[n \tau_{*}^{\beta}\right]+1}^{[n \tau]}\left(G_{i}\left(\beta_{1} \mid \alpha\right)-G_{i}\left(\beta_{2} \mid \alpha\right)-\mathbb{E}_{\beta_{2}^{*}}\left[G_{i}\left(\beta_{1} \mid \alpha\right)-G_{i}\left(\beta_{2} \mid \alpha\right) \mid \mathscr{G}_{i-1}^{n}\right]\right) \\
& +h \sum_{i=\left[n \tau_{*}^{\beta}\right]+1}^{[n \tau]} \operatorname{tr}\left(A_{i-1}^{-1}(\alpha)\left(b_{i-1}\left(\beta_{1}\right)-b_{i-1}\left(\beta_{2}\right)\right)^{\otimes 2}\right) \\
& +2 \sum_{i=\left[n \tau_{*}^{\beta}\right]+1}^{[n \tau]} \operatorname{tr}\left(A_{i-1}^{-1}(\alpha)\left(h b_{i-1}\left(\beta_{2}\right)-\mathbb{E}_{\beta_{2}^{*}}\left[\Delta X_{i} \mid \mathscr{G}_{i-1}^{n}\right]\right)\left(b_{i-1}\left(\beta_{1}\right)-b_{i-1}\left(\beta_{2}\right)\right)^{\top}\right) \\
=: & \mathcal{M}_{n}^{\beta}\left(\tau: \beta_{1}, \beta_{2} \mid \alpha\right)+\mathcal{A}_{n}^{\beta}\left(\tau: \beta_{1}, \beta_{2} \mid \alpha\right)+\varrho_{n}^{\beta}\left(\tau: \beta_{1}, \beta_{2} \mid \alpha\right) .
\end{aligned}
$$

Let $M \geq 1, D_{n, M}^{\beta}=\left\{\tau \in[0,1] \mid T \vartheta_{\beta}^{2}\left(\tau-\tau_{*}^{\beta}\right)>M\right\}$. For all $\delta>0$, we have

$$
\begin{aligned}
P\left(T \vartheta_{\beta}^{2}\left(\hat{\tau}_{n}^{\beta}-\tau_{*}^{\beta}\right)>M\right) \leq & P\left(\inf _{\tau \in D_{n, M}^{\beta}} \Psi_{n}\left(\tau: \hat{\beta}_{1}, \hat{\beta}_{2} \mid \hat{\alpha}\right) \leq \Psi_{n}\left(\tau_{*}^{\beta}: \hat{\beta}_{1}, \hat{\beta}_{2} \mid \hat{\alpha}\right)\right) \\
= & P\left(\inf _{\tau \in D_{n, M}^{\beta}}\left(\Psi_{n}\left(\tau: \hat{\beta}_{1}, \hat{\beta}_{2} \mid \hat{\alpha}\right)-\Psi_{n}\left(\tau_{*}^{\beta}: \hat{\beta}_{1}, \hat{\beta}_{2} \mid \hat{\alpha}\right)\right) \leq 0\right) \\
= & P\left(\inf _{\tau \in D_{n, M}^{\beta}}\left(\mathcal{M}_{n}^{\beta}\left(\tau: \hat{\beta}_{1}, \hat{\beta}_{2} \mid \hat{\alpha}\right)+\mathcal{A}_{n}^{\beta}\left(\tau: \hat{\beta}_{1}, \hat{\beta}_{2} \mid \hat{\alpha}\right)+\varrho_{n}^{\beta}\left(\tau: \hat{\beta}_{1}, \hat{\beta}_{2} \mid \hat{\alpha}\right)\right) \leq 0\right) \\
\leq & P\left(\inf _{\tau \in D_{n, M}^{\beta}} \frac{\mathcal{M}_{n}^{\beta}\left(\tau: \hat{\beta}_{1}, \hat{\beta}_{2} \mid \hat{\alpha}\right)+\mathcal{A}_{n}^{\beta}\left(\tau: \hat{\beta}_{1}, \hat{\beta}_{2} \mid \hat{\alpha}\right)+\varrho_{n}^{\beta}\left(\tau: \hat{\beta}_{1}, \hat{\beta}_{2} \mid \hat{\alpha}\right)}{h \vartheta_{\beta}^{2}\left([n \tau]-\left[n \tau_{*}^{\beta}\right]\right)} \leq 0\right) \\
\leq & P\left(\inf _{\tau \in D_{n, M}^{\beta}} \frac{\mathcal{M}_{n}^{\beta}\left(\tau: \hat{\beta}_{1}, \hat{\beta}_{2} \mid \hat{\alpha}\right)}{h \vartheta_{\beta}^{2}\left([n \tau]-\left[n \tau_{*}^{\beta}\right]\right)} \leq-\delta\right)+P\left(\inf _{\tau \in D_{n, M}^{\beta}} \frac{\mathcal{A}_{n}^{\beta}\left(\tau: \hat{\beta}_{1}, \hat{\beta}_{2} \mid \hat{\alpha}\right)}{h \vartheta_{\beta}^{2}\left([n \tau]-\left[n \tau_{*}^{\beta}\right]\right)} \leq 2 \delta\right) \\
& +P\left(\inf _{\tau \in D_{n, M}^{\beta}} \frac{\varrho_{n}^{\beta}\left(\tau: \hat{\beta}_{1}, \hat{\beta}_{2} \mid \hat{\alpha}\right)}{h \vartheta_{\beta}^{2}\left([n \tau]-\left[n \tau_{*}^{\beta}\right]\right)} \leq-\delta\right) \\
\leq & P\left(\sup _{\tau \in D_{n, M}^{\beta}} \frac{\left|\mathcal{M}_{n}^{\beta}\left(\tau: \hat{\beta}_{1}, \hat{\beta}_{2} \mid \hat{\alpha}\right)\right|}{h \vartheta_{\beta}^{2}\left([n \tau]-\left[n \tau_{*}^{\beta}\right]\right)} \geq \delta\right)+P\left(\inf _{\tau \in D_{n, M}^{\beta}} \frac{\mathcal{A}_{n}^{\beta}\left(\tau: \hat{\beta}_{1}, \hat{\beta}_{2} \mid \hat{\alpha}\right)}{h \vartheta_{\beta}^{2}\left([n \tau]-\left[n \tau_{*}^{\beta}\right]\right)} \leq 2 \delta\right) \\
& +P\left(\sup _{\tau \in D_{n, M}^{\beta}} \frac{\left|\varrho_{n}^{\beta}\left(\tau: \hat{\beta}_{1}, \hat{\beta}_{2} \mid \hat{\alpha}\right)\right|}{h \vartheta_{\beta}^{2}\left([n \tau]-\left[n \tau_{*}^{\beta}\right]\right)} \geq \delta\right) \\
= & P_{1, n}^{\beta}+P_{2, n}^{\beta}+P_{3, n}^{\beta} .
\end{aligned}
$$

[i] Evaluation of $P_{1, n}^{\beta}$. Choose $\epsilon>0$ as you like. Because $\partial_{\beta} G_{i}(\beta \mid \alpha)$ is continuous with respect to $\beta \in \Theta_{B}$, we can choose $\bar{\beta} \in \mathcal{O}_{\hat{\beta}_{2}}$ so that

$$
\begin{aligned}
\mathcal{M}_{n}^{\beta}\left(\tau: \hat{\beta}_{1}, \hat{\beta}_{2} \mid \alpha\right) & =\sum_{i=\left[n \tau_{*}^{\beta}\right]+1}^{[n \tau]}\left(G_{i}\left(\hat{\beta}_{1} \mid \alpha\right)-G_{i}\left(\hat{\beta}_{2} \mid \alpha\right)-\mathbb{E}_{\beta_{2}^{*}}\left[G_{i}\left(\hat{\beta}_{1} \mid \alpha\right)-G_{i}\left(\hat{\beta}_{2} \mid \alpha\right) \mid \mathscr{G}_{i-1}^{n}\right]\right) \\
& =\sum_{i=\left[n \tau \tau_{*}^{\beta}\right]+1}^{[n \tau]}\left(\partial_{\beta} G_{i}(\bar{\beta} \mid \alpha)-\left.\mathbb{E}_{\beta_{2}^{*}}\left[\partial_{\beta} G_{i}(\beta \mid \alpha) \mid \mathscr{G}_{i-1}^{n}\right]\right|_{\beta=\bar{\beta}}\right)\left(\hat{\beta}_{1}-\hat{\beta}_{2}\right) .
\end{aligned}
$$

If $\hat{\alpha} \in \mathcal{O}_{\alpha^{*}}$ and $\hat{\beta}_{k} \in \mathcal{O}_{\beta_{0}}$, then

$$
\left|\mathcal{M}_{n}^{\beta}\left(\tau: \hat{\beta}_{1}, \hat{\beta}_{2} \mid \hat{\alpha}\right)\right| \leq\left|\sum_{i=\left[n \tau_{*}^{\beta}\right]+1}^{[n \tau]}\left(\partial_{\beta} G_{i}(\bar{\beta} \mid \hat{\alpha})-\mathbb{E}_{\beta_{2}^{*}}\left[\partial_{\beta} G_{i}(\bar{\beta} \mid \hat{\alpha}) \mid \mathscr{G}_{i-1}^{n}\right]\right)\right|\left|\hat{\beta}_{1}-\hat{\beta}_{2}\right|
$$




$$
\begin{aligned}
& \leq \sup _{(\alpha, \beta) \in \Theta}\left|\sum_{i=\left[n \tau_{*}^{\beta}\right]+1}^{[n \tau]}\left(\partial_{\beta} G_{i}(\beta \mid \alpha)-\mathbb{E}_{\beta_{2}^{*}}\left[\partial_{\beta} G_{i}(\beta \mid \alpha) \mid \mathscr{G}_{i-1}^{n}\right]\right)\right|\left|\hat{\beta}_{1}-\hat{\beta}_{2}\right| \\
& =: \sup _{(\alpha, \beta) \in \Theta}\left|\mathbb{M}_{n}^{\beta}(\tau: \beta \mid \alpha)\right|\left|\hat{\beta}_{1}-\hat{\beta}_{2}\right| .
\end{aligned}
$$

Hence we have

$$
\begin{aligned}
P_{1, n}^{\beta}= & P\left(\sup _{\tau \in D_{n, M}^{\beta}} \frac{\left|\mathcal{M}_{n}^{\beta}\left(\tau: \hat{\beta}_{1}, \hat{\beta}_{2} \mid \hat{\alpha}\right)\right|}{h \vartheta_{\beta}^{2}\left([n \tau]-\left[n \tau_{*}^{\beta}\right]\right)} \geq \delta\right) \\
\leq & P\left(\sup _{\tau \in D_{n, M}^{\beta}} \frac{\left|\mathcal{M}_{n}^{\beta}\left(\tau: \hat{\beta}_{1}, \hat{\beta}_{2} \mid \hat{\alpha}\right)\right|}{h \vartheta_{\beta}^{2}\left([n \tau]-\left[n \tau_{*}^{\beta}\right]\right)} \geq \delta,\left|\hat{\beta}_{1}-\hat{\beta}_{2}\right| \leq 2 \vartheta_{\beta}, \hat{\alpha} \in \mathcal{O}_{\alpha^{*}}, \hat{\beta}_{k} \in \mathcal{O}_{\beta_{0}}\right) \\
& +P\left(\left|\hat{\beta}_{1}-\hat{\beta}_{2}\right|>2 \vartheta_{\beta}\right)+P\left(\hat{\alpha} \notin \mathcal{O}_{\alpha^{*}}\right)+P\left(\hat{\beta}_{1} \notin \mathcal{O}_{\beta_{0}}\right)+P\left(\hat{\beta}_{2} \notin \mathcal{O}_{\beta_{0}}\right) \\
\leq & P\left(\sup _{\tau \in D_{n, M}^{\beta}} \frac{\sup _{(\alpha, \beta) \in \Theta}\left|\mathbb{M}_{n}^{\beta}(\tau: \beta \mid \alpha)\right|}{h \vartheta_{\beta}^{2}\left([n \tau]-\left[n \tau_{*}^{\beta}\right]\right)}\left|\hat{\beta}_{1}-\hat{\beta}_{2}\right| \geq \delta,\left|\hat{\beta}_{1}-\hat{\beta}_{2}\right| \leq 2 \vartheta_{\beta}\right) \\
& +P\left(\left|\hat{\beta}_{1}-\hat{\beta}_{2}\right|>2 \vartheta_{\beta}\right)+P\left(\hat{\alpha} \notin \mathcal{O}_{\alpha^{*}}\right)+P\left(\hat{\beta}_{1} \notin \mathcal{O}_{\beta_{0}}\right)+P\left(\hat{\beta}_{2} \notin \mathcal{O}_{\beta_{0}}\right) \\
\leq & P\left(\sup _{\tau \in D_{n, M}^{\beta}} \frac{2 \sup _{(\alpha, \beta) \in \Theta}\left|\mathbb{M}_{n}^{\beta}(\tau: \beta \mid \alpha)\right|}{h \vartheta_{\beta}\left([n \tau]-\left[n \tau_{*}^{\beta}\right]\right)} \geq \delta\right) \\
& +P\left(\left|\hat{\beta}_{1}-\hat{\beta}_{2}\right|>2 \vartheta_{\beta}\right)+P\left(\hat{\alpha} \notin \mathcal{O}_{\alpha^{*}}\right)+P\left(\hat{\beta}_{1} \notin \mathcal{O}_{\beta_{0}}\right)+P\left(\hat{\beta}_{2} \notin \mathcal{O}_{\beta_{0}}\right) .
\end{aligned}
$$

By the uniform version on the Hájek-Renyi inequality in Lemma 2 of Iacus and Yoshida (2012), we obtain

$$
\begin{aligned}
& P\left(\sup _{\tau \in D_{n, M}^{\beta}} \frac{2 \sup _{(\alpha, \beta) \in \Theta}\left|\mathbb{M}_{n}^{\beta}(\tau: \beta \mid \alpha)\right|}{h \vartheta_{\beta}\left([n \tau]-\left[n \tau_{*}^{\beta}\right]\right)} \geq \delta\right) \\
& =P\left(\sup _{\tau \in D_{n, M}^{\beta}} \frac{2 \sup _{(\alpha, \beta) \in \Theta}\left|\mathbb{M}_{n}^{\beta}(\tau: \beta \mid \alpha)\right|}{[n \tau]-\left[n \tau_{*}^{\beta}\right]} \geq \delta h \vartheta_{\beta}\right) \\
& \leq P\left(\max _{j>M / h \vartheta_{\beta}^{2}-1} \frac{2}{j} \sup _{(\alpha, \beta) \in \Theta}\left|\sum_{i=\left[n \tau_{*}^{\beta}\right]+1}^{\left[n \tau_{*}^{\beta}\right]+j}\left(\partial_{\beta} G_{i}(\beta \mid \alpha)-\mathbb{E}\left[\partial_{\beta} G_{i}(\beta \mid \alpha) \mid \mathscr{G}_{i-1}^{n}\right]\right)\right| \geq \delta h \vartheta_{\alpha}\right) \\
& \leq \sum_{j>M / h \vartheta_{\beta}^{2}-1} \frac{C h}{\left(\delta h \vartheta_{\beta} j\right)^{2}} \\
& \leq \frac{C^{\prime} h}{\left(\delta h \vartheta_{\beta}\right)^{2}} \frac{h \vartheta_{\beta}^{2}}{M}=\frac{C^{\prime}}{\delta^{2} M}=: \gamma_{\beta}(M) .
\end{aligned}
$$

Noting that if $\left|\hat{\beta}_{k}-\beta_{k}^{*}\right| \leq \vartheta_{\beta} / 2$ holds, then $\left|\hat{\beta}_{1}-\hat{\beta}_{2}\right| \leq\left|\hat{\beta}_{1}-\beta_{1}^{*}\right|+\left|\beta_{1}^{*}-\beta_{2}^{*}\right|+\left|\hat{\beta}_{2}-\beta_{2}^{*}\right| \leq 2 \vartheta_{\beta}$, i.e.,

$$
\left\{\left|\hat{\beta}_{1}-\hat{\beta}_{2}\right|>2 \vartheta_{\beta}\right\} \subset\left\{\left|\hat{\beta}_{1}-\beta_{1}^{*}\right|>\frac{\vartheta_{\beta}}{2}\right\} \cup\left\{\left|\hat{\beta}_{2}-\beta_{2}^{*}\right|>\frac{\vartheta_{\beta}}{2}\right\}
$$

and for sufficiently large $n$ so that $P\left(\left|\hat{\beta}_{k}-\beta_{k}^{*}\right|>\vartheta_{\beta} / 2\right)<\epsilon / 2$ because of $\vartheta_{\beta}^{-1}\left(\hat{\beta}_{k}-\beta_{k}^{*}\right)=o_{p}(1)$, we see

$$
P\left(\left|\hat{\beta}_{1}-\hat{\beta}_{2}\right|>2 \vartheta_{\beta}\right) \leq P\left(\left|\hat{\beta}_{1}-\beta_{1}^{*}\right|>\frac{\vartheta_{\beta}}{2}\right)+P\left(\left|\hat{\beta}_{2}-\beta_{2}^{*}\right|>\frac{\vartheta_{\beta}}{2}\right)<\epsilon .
$$

From [C6-II] and [A2-II], we have $\hat{\alpha} \stackrel{p}{\longrightarrow} \alpha^{*}$ and $\hat{\beta}_{k} \stackrel{p}{\longrightarrow} \beta_{0}$ as $n \rightarrow \infty$, that is, $P\left(\hat{\alpha} \notin \mathcal{O}_{\alpha^{*}}\right)<\epsilon$ and $P\left(\hat{\beta}_{k} \notin \mathcal{O}_{\beta_{0}}\right)<\epsilon$ for large $n$. Therefore, from (7.45)-7.47) and these, we have $P_{1, n}^{\beta} \leq \gamma_{\beta}(M)+4 \epsilon$ for large $n$. 
[ii] Evaluation of $P_{2, n}^{\beta}$. If $\hat{\alpha} \in \mathcal{O}_{\alpha^{*}}$ and $\hat{\beta}_{k} \in \mathcal{O}_{\beta_{0}}$, then we have

$$
\begin{aligned}
& \operatorname{tr}\left(A_{i-1}^{-1}(\hat{\alpha})\left(b_{i-1}\left(\hat{\beta}_{1}\right)-b_{i-1}\left(\hat{\beta}_{2}\right)\right)^{\otimes 2}\right) \\
& =\operatorname{tr}\left(A_{i-1}^{-1}\left(\alpha^{*}\right)\left(b_{i-1}\left(\hat{\beta}_{1}\right)-b_{i-1}\left(\hat{\beta}_{2}\right)\right)^{\otimes 2}\right) \\
& +\left.\int_{0}^{1} \partial_{\alpha} \operatorname{tr}\left(A_{i-1}^{-1}(\alpha)\left(b_{i-1}\left(\hat{\beta}_{1}\right)-b_{i-1}\left(\hat{\beta}_{2}\right)\right)^{\otimes 2}\right)\right|_{\alpha=\alpha^{*}+u\left(\hat{\alpha}-\alpha^{*}\right)} \mathrm{d} u\left(\hat{\alpha}-\alpha^{*}\right) \\
& =\left.\partial_{\beta} \operatorname{tr}\left(A_{i-1}^{-1}\left(\alpha^{*}\right)\left(b_{i-1}(\beta)-b_{i-1}\left(\hat{\beta}_{2}\right)\right)^{\otimes 2}\right)\right|_{\beta=\hat{\beta}_{2}}\left(\hat{\beta}_{1}-\hat{\beta}_{2}\right) \\
& +\left.\frac{1}{2} \partial_{\beta}^{2} \operatorname{tr}\left(A_{i-1}^{-1}\left(\alpha^{*}\right)\left(b_{i-1}(\beta)-b_{i-1}\left(\hat{\beta}_{2}\right)\right)^{\otimes 2}\right)\right|_{\beta=\hat{\beta}_{2}} \otimes\left(\hat{\beta}_{1}-\hat{\beta}_{2}\right)^{\otimes 2} \\
& +\left.\frac{1}{3 !} \partial_{\beta}^{3} \operatorname{tr}\left(A_{i-1}^{-1}\left(\alpha^{*}\right)\left(b_{i-1}(\beta)-b_{i-1}\left(\hat{\beta}_{2}\right)\right)^{\otimes 2}\right)\right|_{\beta=\hat{\beta}_{2}} \otimes\left(\hat{\beta}_{1}-\hat{\beta}_{2}\right)^{\otimes 3} \\
& +\left.\int_{0}^{1} \frac{(1-u)^{3}}{3 !} \partial_{\beta}^{4} \operatorname{tr}\left(A_{i-1}^{-1}\left(\alpha^{*}\right)\left(b_{i-1}(\beta)-b_{i-1}\left(\hat{\beta}_{2}\right)\right)^{\otimes 2}\right)\right|_{\beta=\hat{\beta}_{2}+u\left(\hat{\beta}_{1}-\hat{\beta}_{2}\right)} \mathrm{d} u \otimes\left(\hat{\beta}_{1}-\hat{\beta}_{2}\right)^{\otimes 4} \\
& +\left.\int_{0}^{1} \int_{0}^{1} \partial_{\beta} \partial_{\alpha} \operatorname{tr}\left(A_{i-1}^{-1}(\alpha)\left(b_{i-1}(\beta)-b_{i-1}\left(\hat{\beta}_{2}\right)\right)^{\otimes 2}\right)\right|_{\substack{\alpha=\alpha^{*}+u\left(\hat{\alpha}-\alpha^{*}\right) \\
\beta=\hat{\beta}_{2}+v\left(\hat{\beta}_{1}-\hat{\beta}_{2}\right)}} \mathrm{d} u \mathrm{~d} v \otimes\left(\hat{\alpha}-\alpha^{*}\right) \otimes\left(\hat{\beta}_{1}-\hat{\beta}_{2}\right) \\
& =\Xi_{i-1}^{\beta}\left(\alpha^{*}, \hat{\beta}_{2}\right) \otimes\left(\hat{\beta}_{1}-\hat{\beta}_{2}\right)^{\otimes 2} \\
& +\left.\frac{1}{3 !} \partial_{\beta}^{3} \operatorname{tr}\left(A_{i-1}^{-1}\left(\alpha^{*}\right)\left(b_{i-1}(\beta)-b_{i-1}\left(\hat{\beta}_{2}\right)\right)^{\otimes 2}\right)\right|_{\beta=\hat{\beta}_{2}} \otimes\left(\hat{\beta}_{1}-\hat{\beta}_{2}\right)^{\otimes 3} \\
& +\left.\int_{0}^{1} \frac{(1-u)^{3}}{3 !} \partial_{\beta}^{4} \operatorname{tr}\left(A_{i-1}^{-1}\left(\alpha^{*}\right)\left(b_{i-1}(\beta)-b_{i-1}\left(\hat{\beta}_{2}\right)\right)^{\otimes 2}\right)\right|_{\beta=\hat{\beta}_{2}+u\left(\hat{\beta}_{1}-\hat{\beta}_{2}\right)} \mathrm{d} u \otimes\left(\hat{\beta}_{1}-\hat{\beta}_{2}\right)^{\otimes 4} \\
& +\left.\int_{0}^{1} \int_{0}^{1} \partial_{\beta} \partial_{\alpha} \operatorname{tr}\left(A_{i-1}^{-1}(\alpha)\left(b_{i-1}(\beta)-b_{i-1}\left(\hat{\beta}_{2}\right)\right)^{\otimes 2}\right)\right|_{\substack{\alpha=\alpha^{*}+u\left(\hat{\alpha}-\alpha^{*}\right) \\
\beta=\hat{\beta}_{2}+v\left(\hat{\beta}_{1}-\hat{\beta}_{2}\right)}} \mathrm{d} u \mathrm{~d} v \otimes\left(\hat{\alpha}-\alpha^{*}\right) \otimes\left(\hat{\beta}_{1}-\hat{\beta}_{2}\right) \\
& =\Xi_{i-1}^{\beta}\left(\alpha^{*}, \beta_{0}\right) \otimes\left(\hat{\beta}_{1}-\hat{\beta}_{2}\right)^{\otimes 2}+\partial_{\beta} \Xi_{i-1}^{\beta}\left(\alpha^{*}, \beta_{0}\right) \otimes\left(\hat{\beta}_{1}-\hat{\beta}_{2}\right)^{\otimes 2} \otimes\left(\hat{\beta}_{2}-\beta_{0}\right) \\
& +\int_{0}^{1}(1-u) \partial_{\beta}^{2} \Xi_{i-1}^{\beta}\left(\alpha^{*}, \beta_{0}+u\left(\hat{\beta}_{2}-\beta_{0}\right)\right) \mathrm{d} u \otimes\left(\hat{\beta}_{1}-\hat{\beta}_{2}\right)^{\otimes 2} \otimes\left(\hat{\beta}_{2}-\beta_{0}\right)^{\otimes 2} \\
& +\left.\frac{1}{3 !} \partial_{\beta}^{3} \operatorname{tr}\left(A_{i-1}^{-1}\left(\alpha^{*}\right)\left(b_{i-1}(\beta)-b_{i-1}\left(\hat{\beta}_{2}\right)\right)^{\otimes 2}\right)\right|_{\beta=\hat{\beta}_{2}} \otimes\left(\hat{\beta}_{1}-\hat{\beta}_{2}\right)^{\otimes 3} \\
& +\left.\int_{0}^{1} \frac{(1-u)^{3}}{3 !} \partial_{\beta}^{4} \operatorname{tr}\left(A_{i-1}^{-1}\left(\alpha^{*}\right)\left(b_{i-1}(\beta)-b_{i-1}\left(\hat{\beta}_{2}\right)\right)^{\otimes 2}\right)\right|_{\beta=\hat{\beta}_{2}+u\left(\hat{\beta}_{1}-\hat{\beta}_{2}\right)} \mathrm{d} u \otimes\left(\hat{\beta}_{1}-\hat{\beta}_{2}\right)^{\otimes 4} \\
& +\left.\int_{0}^{1} \int_{0}^{1} \partial_{\beta} \partial_{\alpha} \operatorname{tr}\left(A_{i-1}^{-1}(\alpha)\left(b_{i-1}(\beta)-b_{i-1}\left(\hat{\beta}_{2}\right)\right)^{\otimes 2}\right)\right|_{\substack{\alpha=\alpha^{*}+u\left(\hat{\alpha}-\alpha^{*}\right) \\
\beta=\hat{\beta}_{2}+v\left(\hat{\beta}_{1}-\hat{\beta}_{2}\right)}} \mathrm{d} u \mathrm{~d} v \otimes\left(\hat{\alpha}-\alpha^{*}\right) \otimes\left(\hat{\beta}_{1}-\hat{\beta}_{2}\right) \\
& \geq\left(\lambda_{1}\left[\Xi_{i-1}^{\beta}\left(\alpha^{*}, \beta_{0}\right)\right]+r_{i-1}\right)\left|\hat{\beta}_{1}-\hat{\beta}_{2}\right|^{2},
\end{aligned}
$$

where $r_{i-1}$ satisfies

$$
\sup _{\tau \in D_{n, M}^{\beta}}\left|\frac{1}{[n \tau]-\left[n \tau_{*}^{\beta}\right]} \sum_{i=\left[n \tau_{*}^{\beta}\right]+1}^{[n \tau]} r_{i-1}\right|=o_{p}(1)
$$

from [A1-II $]$ and [A2-II]. Therefore we have

$$
\begin{aligned}
P_{2, n}^{\beta}= & P\left(\inf _{\tau \in D_{n, M}^{\beta}} \frac{\mathcal{A}_{n}^{\beta}\left(\tau: \hat{\beta}_{1}, \hat{\beta}_{2} \mid \hat{\alpha}\right)}{h \vartheta_{\beta}^{2}\left([n \tau]-\left[n \tau_{*}^{\beta}\right]\right)} \leq 2 \delta\right) \\
\leq & P\left(\inf _{\tau \in D_{n, M}^{\beta}} \frac{\mathcal{A}_{n}^{\beta}\left(\tau: \hat{\beta}_{1}, \hat{\beta}_{2} \mid \hat{\alpha}\right)}{h \vartheta_{\beta}^{2}\left([n \tau]-\left[n \tau_{*}^{\beta}\right]\right)} \leq 2 \delta,\left|\hat{\beta}_{1}-\hat{\beta}_{2}\right| \geq \frac{\vartheta_{\beta}}{2}, \hat{\alpha} \in \mathcal{O}_{\alpha^{*}}, \hat{\beta}_{k} \in \mathcal{O}_{\beta_{0}}\right) \\
& +P\left(\left|\hat{\beta}_{1}-\hat{\beta}_{2}\right|<\frac{\vartheta_{\beta}}{2}\right)+P\left(\hat{\alpha} \notin \mathcal{O}_{\alpha^{*}}\right)+P\left(\hat{\beta}_{1} \notin \mathcal{O}_{\beta_{0}}\right)+P\left(\hat{\beta}_{2} \notin \mathcal{O}_{\beta_{0}}\right)
\end{aligned}
$$




$$
\begin{aligned}
\leq & P\left(\inf _{\tau \in D_{n, M}^{\beta}} \frac{1}{\vartheta_{\beta}^{2}\left([n \tau]-\left[n \tau_{*}^{\beta}\right]\right)} \sum_{i=\left[n \tau_{*}^{\beta}\right]+1}^{[n \tau]}\left(\lambda_{1}\left[\Xi_{i-1}^{\beta}\left(\alpha^{*}, \beta_{0}\right)\right]+r_{i-1}\right)\left|\hat{\beta}_{1}-\hat{\beta}_{2}\right|^{2} \leq 2 \delta,\left|\hat{\beta}_{1}-\hat{\beta}_{2}\right| \geq \frac{\vartheta_{\beta}}{2}\right) \\
& +P\left(\left|\hat{\beta}_{1}-\hat{\beta}_{2}\right|<\frac{\vartheta_{\beta}}{2}\right)+P\left(\hat{\alpha} \notin \mathcal{O}_{\alpha^{*}}\right)+P\left(\hat{\beta}_{1} \notin \mathcal{O}_{\beta_{0}}\right)+P\left(\hat{\beta}_{2} \notin \mathcal{O}_{\beta_{0}}\right) \\
\leq & P\left(\inf _{\tau \in D_{n, M}^{\beta}} \frac{1}{[n \tau]-\left[n \tau_{*}^{\beta}\right]} \sum_{i=\left[n \tau_{*}^{\beta}\right]+1}^{[n \tau]}\left(\lambda_{1}\left[\Xi_{i-1}^{\beta}\left(\beta_{0}\right)\right]+r_{i-1}\right) \leq 8 \delta\right) \\
& +P\left(\left|\hat{\beta}_{1}-\hat{\beta}_{2}\right|<\frac{\vartheta_{\beta}}{2}\right)+P\left(\hat{\alpha} \notin \mathcal{O}_{\alpha^{*}}\right)+P\left(\hat{\beta}_{1} \notin \mathcal{O}_{\beta_{0}}\right)+P\left(\hat{\beta}_{2} \notin \mathcal{O}_{\beta_{0}}\right) .
\end{aligned}
$$

According to [A2-II], if we set

$$
\delta=\frac{1}{10} \int_{\mathbb{R}^{d}} \lambda_{1}\left[\Xi^{\beta}\left(x, \alpha^{*}, \beta_{0}\right)\right] \mathrm{d} \mu_{\left(\alpha^{*}, \beta_{0}\right)}(x)>0,
$$

then for large $n$,

$$
\begin{aligned}
& P\left(\inf _{\tau \in D_{n, M}^{\beta}} \frac{1}{[n \tau]-\left[n \tau_{*}^{\beta}\right]} \sum_{i=\left[n \tau_{*}^{\beta}\right]+1}^{[n \tau]}\left(\lambda_{1}\left[\Xi_{i-1}^{\beta}\left(\alpha^{*}, \beta_{0}\right)\right]+r_{i-1}\right) \leq 8 \delta\right) \\
& \leq P\left(\inf _{\tau \in D_{n, M}^{\beta}} \frac{1}{[n \tau]-\left[n \tau_{*}^{\beta}\right]} \sum_{i=\left[n \tau_{*}^{\beta}\right]+1}^{[n \tau]} \lambda_{1}\left[\Xi_{i-1}^{\beta}\left(\alpha^{*}, \beta_{0}\right)\right] \leq 9 \delta\right)+P\left(\inf _{\tau \in D_{n, M}^{\beta}} \frac{1}{[n \tau]-\left[n \tau_{*}^{\beta}\right]} \sum_{i=\left[n \tau_{*}^{\beta}\right]+1}^{[n \tau]} r_{i-1} \leq-\delta\right) \\
& \leq P\left(\inf _{\tau \in D_{n, M}^{\beta}} \frac{1}{[n \tau]-\left[n \tau_{*}^{\beta}\right]} \sum_{i=\left[n \tau_{*}^{\beta}\right]+1}^{[n \tau]} \lambda_{1}\left[\Xi_{i-1}^{\beta}\left(\alpha^{*}, \beta_{0}\right)\right] \leq 9 \delta\right)+P\left(\sup _{\tau \in D_{n, M}^{\beta}}\left|\frac{1}{[n \tau]-\left[n \tau_{*}^{\beta}\right]} \sum_{i=\left[n \tau_{*}^{\beta}\right]+1}^{[n \tau]} r_{i-1}\right| \geq \delta\right) \\
& \leq P\left(\inf _{\tau \in D_{n, M}^{\beta}}\left(\frac{1}{[n \tau]-\left[n \tau_{*}^{\beta}\right]} \sum_{i=\left[n \tau_{*}^{\beta}\right]+1}^{[n \tau]} \lambda_{1}\left[\Xi_{i-1}^{\beta}\left(\alpha^{*}, \beta_{0}\right)\right]-10 \delta\right) \leq-\delta\right)+\epsilon \\
& \leq P\left(\sup _{\tau \in D_{n, M}^{\beta}}\left|\frac{1}{[n \tau]-\left[n \tau_{*}^{\beta}\right]} \sum_{i=\left[n \tau_{*}^{\beta}\right]+1}^{[n \tau]} \lambda_{1}\left[\Xi_{i-1}^{\beta}\left(\alpha^{*}, \beta_{0}\right)\right]-10 \delta\right| \geq \delta\right)+\epsilon \\
& \leq P\left(\sup _{k>M / h \vartheta_{\beta}^{2}-1}\left|\frac{1}{k} \sum_{i=\left[n \tau_{*}^{\beta}\right]+1}^{\left[n \tau_{*}^{\beta}\right]+k} \lambda_{1}\left[\Xi_{i-1}^{\beta}\left(\alpha^{*}, \beta_{0}\right)\right]-10 \delta\right| \geq \delta\right)+\epsilon \\
& \leq P\left(\max _{\left[n^{1 / r}\right] \leq k \leq n}\left|\frac{1}{k} \sum_{i=\left[n \tau_{*}^{\beta}\right]+1}^{\left[n \tau_{*}^{\beta}\right]+k} \lambda_{1}\left[\Xi_{i-1}^{\beta}\left(\alpha^{*}, \beta_{0}\right)\right]-10 \delta\right| \geq \delta\right)+\epsilon \\
& \leq 2 \epsilon \text {. }
\end{aligned}
$$

Noting that if $\left|\hat{\beta}_{k}-\beta_{k}^{*}\right| \leq \vartheta_{\beta} / 4$ holds, then $\vartheta_{\beta}=\left|\beta_{1}^{*}-\beta_{2}^{*}\right| \leq\left|\beta_{1}^{*}-\hat{\beta}_{1}\right|+\left|\hat{\beta}_{1}-\hat{\beta}_{2}\right|+\left|\hat{\beta}_{2}-\beta_{2}^{*}\right| \leq\left|\hat{\beta}_{1}-\hat{\beta}_{2}\right|+\vartheta_{\beta} / 2$, i.e.,

$$
\left\{\left|\hat{\beta}_{1}-\hat{\beta}_{2}\right|<\frac{\vartheta_{\beta}}{2}\right\} \subset\left\{\left|\hat{\beta}_{1}-\beta_{1}^{*}\right|>\frac{\vartheta_{\beta}}{4}\right\} \cup\left\{\left|\hat{\beta}_{2}-\beta_{2}^{*}\right|>\frac{\vartheta_{\beta}}{4}\right\}
$$

and for sufficiently large $n$ so that $P\left(\left|\hat{\beta}_{k}-\beta_{k}^{*}\right|>\vartheta_{\beta} / 4\right)<\epsilon / 2$ because of $\vartheta_{\beta}^{-1}\left(\hat{\beta}_{k}-\beta_{k}^{*}\right)=o_{p}(1)$, we see

$$
P\left(\left|\hat{\beta}_{1}-\hat{\beta}_{2}\right|<\frac{\vartheta_{\beta}}{2}\right) \leq P\left(\left|\hat{\beta}_{1}-\beta_{1}^{*}\right|>\frac{\vartheta_{\beta}}{4}\right)+P\left(\left|\hat{\beta}_{2}-\beta_{2}^{*}\right|>\frac{\vartheta_{\beta}}{4}\right)<\epsilon .
$$

Therefore, from 7.48-7.50, we obtain $P_{2, n}^{\beta} \leq 6 \epsilon$ for large $n$. 
[iii] Evaluation of $P_{3, n}^{\beta}$. We have, for large $n$,

$$
\begin{aligned}
& \operatorname{tr}\left(A_{i-1}^{-1}(\hat{\alpha})\left(b_{i-1}\left(\hat{\beta}_{2}\right)-h \mathbb{E}\left[\Delta X_{i} \mid \mathscr{G}_{i-1}^{n}\right]\right)\left(b_{i-1}\left(\hat{\beta}_{1}\right)-b_{i-1}\left(\hat{\beta}_{2}\right)\right)^{\top}\right) \\
& \leq h \operatorname{tr}\left(A_{i-1}^{-1}(\hat{\alpha})\left(b_{i-1}\left(\hat{\beta}_{2}\right)-b_{i-1}\left(\beta_{2}^{*}\right)\right)\left(b_{i-1}\left(\hat{\beta}_{1}\right)-b_{i-1}\left(\hat{\beta}_{2}\right)\right)^{\top}\right)+R_{i-1}\left(h^{2}, \theta\right)\left|b_{i-1}\left(\hat{\beta}_{1}\right)-b_{i-1}\left(\hat{\beta}_{2}\right)\right| \\
& =h\left[\operatorname{tr}\left(A_{i-1}^{-1}(\hat{\alpha}) \partial_{\beta^{\ell_{1}}} b_{i-1}\left(\beta_{2}^{*}\right)\left(b_{i-1}\left(\hat{\beta}_{1}\right)-b_{i-1}\left(\hat{\beta}_{2}\right)\right)^{\top}\right)\right]_{\ell_{1}}\left(\hat{\beta}_{2}-\beta_{2}^{*}\right) \\
& +\left.h \int_{0}^{1}(1-u)\left[\operatorname{tr}\left(A_{i-1}^{-1}(\hat{\alpha}) \partial_{\beta^{\ell_{2}}} \partial_{\beta^{\ell_{1}}} b_{i-1}(\beta)\left(b_{i-1}\left(\hat{\beta}_{1}\right)-b_{i-1}\left(\hat{\beta}_{2}\right)\right)^{\top}\right)\right]_{\ell_{1}, \ell_{2}}\right|_{\beta=\beta_{2}^{*}+u\left(\hat{\beta}_{2}-\beta_{2}^{*}\right)} \mathrm{d} u \\
& \otimes\left(\hat{\beta}_{2}-\beta_{2}^{*}\right)^{\otimes 2}+R_{i-1}\left(h^{2}, \theta\right)\left|b_{i-1}\left(\hat{\beta}_{1}\right)-b_{i-1}\left(\hat{\beta}_{2}\right)\right| \\
& =h\left[\operatorname{tr}\left(A_{i-1}^{-1}(\hat{\alpha}) \partial_{\beta^{\ell_{1}}} b_{i-1}\left(\beta_{2}^{*}\right) \partial_{\beta^{\ell_{2}}} b_{i-1}\left(\hat{\beta}_{2}\right)^{\top}\right)\right]_{\ell_{1}, \ell_{2}} \otimes\left(\hat{\beta}_{2}-\beta_{2}^{*}\right) \otimes\left(\hat{\beta}_{1}-\hat{\beta}_{2}\right) \\
& +\left.h \int_{0}^{1}(1-u)\left[\operatorname{tr}\left(A_{i-1}^{-1}(\hat{\alpha}) \partial_{\beta^{\ell_{1}}} b_{i-1}\left(\beta_{2}^{*}\right) \partial_{\beta^{\ell_{3}}} \partial_{\beta^{\ell_{2}}} b_{i-1}(\beta)^{\top}\right)\right]_{\ell_{1}, \ell_{2}, \ell_{3}}\right|_{\beta=\hat{\beta}_{2}+u\left(\hat{\beta}_{1}-\hat{\beta}_{2}\right)} \mathrm{d} u \\
& \otimes\left(\hat{\beta}_{2}-\beta_{2}^{*}\right) \otimes\left(\hat{\beta}_{1}-\hat{\beta}_{2}\right)^{\otimes 2} \\
& +\left.h \int_{0}^{1}(1-u)\left[\operatorname{tr}\left(A_{i-1}^{-1}(\hat{\alpha}) \partial_{\beta^{\ell_{2}}} \partial_{\beta^{\ell_{1}}} b_{i-1}(\beta)\left(b_{i-1}\left(\hat{\beta}_{1}\right)-b_{i-1}\left(\hat{\beta}_{2}\right)\right)^{\top}\right)\right]_{\ell_{1}, \ell_{2}}\right|_{\beta=\beta_{2}^{*}+u\left(\hat{\beta}_{2}-\beta_{2}^{*}\right)} \mathrm{d} u \\
& \otimes\left(\hat{\beta}_{2}-\beta_{2}^{*}\right)^{\otimes 2}+R_{i-1}\left(h^{2}, \theta\right)\left|b_{i-1}\left(\hat{\beta}_{1}\right)-b_{i-1}\left(\hat{\beta}_{2}\right)\right| \\
& =h \Xi_{i-1}^{\beta}\left(\hat{\alpha}, \beta_{2}^{*}\right) \otimes\left(\hat{\beta}_{2}-\beta_{2}^{*}\right) \otimes\left(\hat{\beta}_{1}-\hat{\beta}_{2}\right) \\
& +h \int_{0}^{1}\left[\operatorname{tr}\left(A_{i-1}^{-1}(\hat{\alpha}) \partial_{\beta^{\ell_{1}}} b_{i-1}\left(\beta_{2}^{*}\right) \partial_{\beta^{\ell_{3}}} \partial_{\beta^{\ell_{2}}} b_{i-1}\left(\beta_{2}^{*}+u\left(\hat{\beta}_{2}-\beta_{2}^{*}\right)\right)^{\top}\right)\right]_{\ell_{1}, \ell_{2}, \ell_{3}} \mathrm{~d} u \\
& \otimes\left(\hat{\beta}_{2}-\beta_{2}^{*}\right)^{\otimes 2} \otimes\left(\hat{\beta}_{1}-\hat{\beta}_{2}\right) \\
& +h \int_{0}^{1}(1-u)\left[\operatorname{tr}\left(A_{i-1}^{-1}(\hat{\alpha}) \partial_{\beta^{\ell_{1}}} b_{i-1}\left(\beta_{2}^{*}\right) \partial_{\beta^{\ell_{3}}} \partial_{\beta^{\ell_{2}}} b_{i-1}\left(\hat{\beta}_{2}+u\left(\hat{\beta}_{1}-\hat{\beta}_{2}\right)\right)^{\top}\right)\right]_{\ell_{1}, \ell_{2}, \ell_{3}} \mathrm{~d} u \\
& \otimes\left(\hat{\beta}_{2}-\beta_{2}^{*}\right) \otimes\left(\hat{\beta}_{1}-\hat{\beta}_{2}\right)^{\otimes 2} \\
& +h \int_{0}^{1}(1-u)\left[\operatorname{tr}\left(A_{i-1}^{-1}(\hat{\alpha}) \partial_{\beta^{\ell_{2}}} \partial_{\beta^{\ell_{1}}} b_{i-1}\left(\beta_{2}^{*}+u\left(\hat{\beta}_{2}-\beta_{2}^{*}\right)\right)\left(b_{i-1}\left(\hat{\beta}_{1}\right)-b_{i-1}\left(\hat{\beta}_{2}\right)\right)^{\top}\right)\right]_{\ell_{1}, \ell_{2}} \mathrm{~d} u \\
& \otimes\left(\hat{\beta}_{2}-\beta_{2}^{*}\right)^{\otimes 2}+R_{i-1}\left(h^{2}, \theta\right)\left|b_{i-1}\left(\hat{\beta}_{1}\right)-b_{i-1}\left(\hat{\beta}_{2}\right)\right| \\
& =h \Xi_{i-1}^{\beta}\left(\alpha^{*}, \beta_{0}\right) \otimes\left(\hat{\beta}_{2}-\beta_{2}^{*}\right) \otimes\left(\hat{\beta}_{1}-\hat{\beta}_{2}\right) \\
& +\left.h \int_{0}^{1} \partial_{(\alpha, \beta)} \Xi_{i-1}^{\beta}(\alpha, \beta)\right|_{\substack{\alpha=\alpha^{*}+u\left(\hat{\alpha}-\alpha^{*}\right) \\
\beta=\beta_{0}+u\left(\beta_{2}^{*}-\beta_{0}\right)}} \mathrm{d} u \otimes\left(\hat{\beta}_{2}-\beta_{2}^{*}\right) \otimes\left(\hat{\beta}_{1}-\hat{\beta}_{2}\right) \otimes\left(\begin{array}{c}
\hat{\alpha}-\alpha^{*} \\
\beta_{2}^{*}-\beta_{0}
\end{array}\right) \\
& +h \int_{0}^{1}\left[\operatorname{tr}\left(A_{i-1}^{-1}(\hat{\alpha}) \partial_{\beta^{\ell_{1}}} b_{i-1}\left(\beta_{2}^{*}\right) \partial_{\beta^{\ell_{3}}} \partial_{\beta^{\ell_{2}}} b_{i-1}\left(\beta_{2}^{*}+u\left(\hat{\beta}_{2}-\beta_{2}^{*}\right)\right)^{\top}\right)\right]_{\ell_{1}, \ell_{2}, \ell_{3}} \mathrm{~d} u \\
& \otimes\left(\hat{\beta}_{2}-\beta_{2}^{*}\right)^{\otimes 2} \otimes\left(\hat{\beta}_{1}-\hat{\beta}_{2}\right) \\
& +h \int_{0}^{1}(1-u)\left[\operatorname{tr}\left(A_{i-1}^{-1}(\hat{\alpha}) \partial_{\beta^{\ell_{1}}} b_{i-1}\left(\beta_{2}^{*}\right) \partial_{\beta^{\ell_{3}}} \partial_{\beta^{\ell_{2}}} b_{i-1}\left(\hat{\beta}_{2}+u\left(\hat{\beta}_{1}-\hat{\beta}_{2}\right)\right)^{\top}\right)\right]_{\ell_{1}, \ell_{2}, \ell_{3}} \mathrm{~d} u \\
& \otimes\left(\hat{\beta}_{2}-\beta_{2}^{*}\right) \otimes\left(\hat{\beta}_{1}-\hat{\beta}_{2}\right)^{\otimes 2} \\
& +h \int_{0}^{1}(1-u)\left[\operatorname{tr}\left(A_{i-1}^{-1}(\hat{\alpha}) \partial_{\beta^{\ell_{2}}} \partial_{\beta^{\ell_{1}}} b_{i-1}\left(\beta_{2}^{*}+u\left(\hat{\beta}_{2}-\beta_{2}^{*}\right)\right)\left(b_{i-1}\left(\hat{\beta}_{1}\right)-b_{i-1}\left(\hat{\beta}_{2}\right)\right)^{\top}\right)\right]_{\ell_{1}, \ell_{2}} \mathrm{~d} u \\
& \otimes\left(\hat{\beta}_{2}-\beta_{2}^{*}\right)^{\otimes 2}+R_{i-1}\left(h^{2}, \theta\right)\left|b_{i-1}\left(\hat{\beta}_{1}\right)-b_{i-1}\left(\hat{\beta}_{2}\right)\right| \\
& \leq h \Xi_{i-1}^{\beta}\left(\alpha^{*}, \beta_{0}\right) \otimes\left(\hat{\beta}_{2}-\beta_{2}^{*}\right) \otimes\left(\hat{\beta}_{1}-\hat{\beta}_{2}\right) \\
& +\frac{h \vartheta_{\beta}}{\sqrt{T}}\left|\int_{0}^{1} \partial_{(\alpha, \beta)} \Xi_{i-1}^{\beta}(\alpha, \beta)\right|_{\substack{\alpha=\alpha^{*}+u\left(\hat{\alpha}-\alpha^{*}\right) \\
\beta=\beta_{0}+u\left(\beta_{2}^{*}-\beta_{0}\right)}} \mathrm{d} u|\sqrt{T}| \hat{\beta}_{2}-\beta_{2}^{*}\left|\vartheta_{\beta}^{-1}\right| \hat{\beta}_{1}-\hat{\beta}_{2} \mid\left(\left|\hat{\alpha}-\alpha^{*}\right|+\left|\beta_{2}^{*}-\beta_{0}\right|\right)
\end{aligned}
$$




$$
\begin{aligned}
& +\frac{h \vartheta_{\beta}}{T}\left|\int_{0}^{1}\left[\operatorname{tr}\left(A_{i-1}^{-1}(\hat{\alpha}) \partial_{\beta^{\ell_{1}}} b_{i-1}\left(\beta_{2}^{*}\right) \partial_{\beta^{\ell_{3}}} \partial_{\beta^{\ell_{2}}} b_{i-1}\left(\beta_{2}^{*}+u\left(\hat{\beta}_{2}-\beta_{2}^{*}\right)\right)^{T}\right)\right]_{\ell_{1}, \ell_{2}, \ell_{3}} \mathrm{~d} u\right| \\
& \times\left(\sqrt{T}\left|\hat{\beta}_{2}-\beta_{2}^{*}\right|\right)^{2} \vartheta_{\beta}^{-1}\left|\hat{\beta}_{1}-\hat{\beta}_{2}\right| \\
& +\frac{h \vartheta_{\beta}^{2}}{\sqrt{T}}\left|\int_{0}^{1}(1-u)\left[\operatorname{tr}\left(A_{i-1}^{-1}(\hat{\alpha}) \partial_{\beta^{\ell_{1}}} b_{i-1}\left(\beta_{2}^{*}\right) \partial_{\beta^{\ell_{3}}} \partial_{\beta^{\ell_{2}}} b_{i-1}\left(\hat{\beta}_{2}+u\left(\hat{\beta}_{1}-\hat{\beta}_{2}\right)\right)^{\top}\right)\right]_{\ell_{1}, \ell_{2}, \ell_{3}} \mathrm{~d} u\right| \\
& \times \sqrt{T}\left|\hat{\beta}_{2}-\beta_{2}^{*}\right|\left(\vartheta_{\beta}^{-1}\left|\hat{\beta}_{1}-\hat{\beta}_{2}\right|\right)^{2} \\
& +\frac{h \vartheta_{\beta}}{T} \mid \int_{0}^{1} \int_{0}^{1}(1-u)\left[\operatorname { t r } \left(A_{i-1}^{-1}(\hat{\alpha}) \partial_{\beta^{\ell_{2}}} \partial_{\beta^{\ell_{1}}} b_{i-1}\left(\beta_{2}^{*}+u\left(\hat{\beta}_{2}-\beta_{2}^{*}\right)\right)\right.\right. \\
& \left.\left.\times \partial_{\beta^{\ell_{3}}} b_{i-1}\left(\hat{\beta}_{2}+v\left(\hat{\beta}_{1}-\hat{\beta}_{2}\right)\right)^{\top}\right)\right]_{\ell_{1}, \ell_{2}, \ell_{3}} \mathrm{~d} u \mathrm{~d} v\left|\left(\sqrt{T}\left|\hat{\beta}_{2}-\beta_{2}^{*}\right|\right)^{2} \vartheta_{\beta}^{-1}\right| \hat{\beta}_{1}-\hat{\beta}_{2} \mid \\
& +R_{i-1}\left(h^{2}, \theta\right)\left|b_{i-1}\left(\hat{\beta}_{1}\right)-b_{i-1}\left(\hat{\beta}_{2}\right)\right| \\
& \leq \frac{h \vartheta_{\beta}}{\sqrt{T}} \Xi_{i-1}^{\beta}\left(\alpha^{*}, \beta_{0}\right) \otimes \sqrt{T}\left(\hat{\beta}_{2}-\beta_{2}^{*}\right) \otimes \vartheta_{\beta}^{-1}\left(\hat{\beta}_{1}-\hat{\beta}_{2}\right)+\left(\frac{h \vartheta_{\beta}}{\sqrt{n T}}+\frac{h \vartheta_{\beta}^{2}}{\sqrt{T}}+\frac{h \vartheta_{\beta}}{T}+h^{2}\right) R_{i-1}(1, \theta)
\end{aligned}
$$

and

$$
\begin{aligned}
\sup _{\tau \in D_{n, M}^{\beta}} \frac{\left|\varrho_{n}^{\beta}\left(\tau: \hat{\beta}_{1}, \hat{\beta}_{2} \mid \hat{\alpha}\right)\right|}{h \vartheta_{\beta}^{2}\left([n \tau]-\left[n \tau_{*}^{\beta}\right]\right)} \leq & \frac{1}{\sqrt{T} \vartheta_{\beta}} \sup _{\tau \in D_{n, M}^{\beta}}\left|\frac{1}{[n \tau]-\left[n \tau_{*}^{\beta}\right]} \sum_{i=\left[n \tau_{*}^{\beta}\right]+1}^{[n \tau]} \Xi_{i-1}^{\beta}\left(\alpha^{*}, \beta_{0}\right)\right| \sqrt{T}\left|\hat{\beta}_{2}-\beta_{2}^{*}\right| \vartheta_{\beta}^{-1}\left|\hat{\beta}_{1}-\hat{\beta}_{2}\right| \\
& +\left(\frac{h \vartheta_{\beta}}{\sqrt{n T}}+\frac{h \vartheta_{\beta}^{2}}{\sqrt{T}}+\frac{h \vartheta_{\beta}}{T}+h^{2}\right) \sup _{\tau \in D_{n, M}^{\beta}} \frac{1}{h \vartheta_{\beta}^{2}\left([n \tau]-\left[n \tau_{*}^{\beta}\right]\right)} \sum_{i=\left[n \tau_{*}^{\beta}\right]+1}^{[n \tau]} R_{i-1}(1, \theta) \\
\leq & O_{p}\left(\frac{1}{\sqrt{T} \vartheta_{\beta}}\right)+O_{p}\left(\sqrt{h} \vartheta_{\beta}\right)+O_{p}\left(\sqrt{T} \vartheta_{\beta}^{2}\right)+O_{p}\left(\vartheta_{\beta}\right)+O_{p}\left(n h^{2}\right)=o_{p}(1) .
\end{aligned}
$$

Hence, we see

$$
\begin{aligned}
P_{3, n}^{\beta} \leq & P\left(\sup _{\tau \in D_{n, M}^{\beta}} \frac{\left|\varrho_{n}^{\beta}\left(\tau: \hat{\beta}_{1}, \hat{\beta}_{2} \mid \hat{\alpha}\right)\right|}{h \vartheta_{\beta}^{2}\left([n \tau]-\left[n \tau_{*}^{\beta}\right]\right)} \geq \delta, \hat{\alpha} \in \mathcal{O}_{\alpha^{*}}, \hat{\beta}_{1}, \hat{\beta}_{2} \in \mathcal{O}_{\beta_{0}}\right) \\
& \quad+P\left(\hat{\alpha} \notin \mathcal{O}_{\alpha^{*}}\right)+P\left(\hat{\beta}_{1} \notin \mathcal{O}_{\beta_{0}}\right)+P\left(\hat{\beta}_{2} \notin \mathcal{O}_{\beta_{0}}\right) \\
\leq 4 \epsilon &
\end{aligned}
$$

for large $n$.

[iv] From the evaluations in Steps [i]-[iii], we have

$$
\varlimsup_{n \rightarrow \infty} P\left(T \vartheta_{\beta}^{2}\left(\hat{\tau}_{n}^{\beta}-\tau_{*}^{\beta}\right)>M\right) \leq \gamma_{\beta}(M)+14 \epsilon
$$

for any $M \geq 1$ and $\epsilon>0$. Therefore

$$
\varlimsup_{M \rightarrow \infty} \varlimsup_{n \rightarrow \infty} P\left(T \vartheta_{\beta}^{2}\left(\hat{\tau}_{n}^{\beta}-\tau_{*}^{\beta}\right)>M\right) \leq 14 \epsilon .
$$

Proof of Theorem 4. It is sufficient to show

$$
\varlimsup_{M \rightarrow \infty} \varlimsup_{n \rightarrow \infty} P\left(T\left(\hat{\tau}_{n}^{\beta}-\tau_{*}^{\beta}\right)>M\right)=0 .
$$

Let $M \geq 1, D_{n, M}^{\beta}=\left\{\tau \in[0,1] \mid T\left(\tau-\tau_{*}^{\beta}\right)>M\right\}$. For all $\delta>0$, we have

$$
P\left(T\left(\hat{\tau}_{n}^{\beta}-\tau_{*}^{\beta}\right)>M\right) \leq P\left(\sup _{\tau \in D_{n, M}^{\beta}} \frac{\left|\mathcal{M}_{n}^{\beta}\left(\tau: \hat{\beta}_{1}, \hat{\beta}_{2} \mid \hat{\alpha}\right)\right|}{h\left([n \tau]-\left[n \tau_{*}^{\beta}\right]\right)} \geq \delta\right)+P\left(\inf _{\tau \in D_{n, M}^{\beta}} \frac{\mathcal{A}_{n}^{\beta}\left(\tau: \hat{\beta}_{1}, \hat{\beta}_{2} \mid \hat{\alpha}\right)}{h\left([n \tau]-\left[n \tau_{*}^{\beta}\right]\right)} \leq 2 \delta\right)
$$




$$
\begin{aligned}
& +P\left(\sup _{\tau \in D_{n, M}^{\beta}} \frac{\left|\varrho_{n}^{\beta}\left(\tau: \hat{\beta}_{1}, \hat{\beta}_{2} \mid \hat{\alpha}\right)\right|}{h\left([n \tau]-\left[n \tau_{*}^{\beta}\right]\right)} \geq \delta\right) \\
= & : P_{1, n}^{\beta}+P_{2, n}^{\beta}+P_{3, n}^{\beta} .
\end{aligned}
$$

[i] Evaluation of $P_{1, n}^{\beta}$. For large $n$, we have

$$
\begin{aligned}
& P_{1, n}^{\beta} \leq P\left(\sup _{\tau \in D_{n, M}^{\beta}} \frac{\left|\mathcal{M}_{n}^{\beta}\left(\tau: \hat{\beta}_{1}, \hat{\beta}_{2} \mid \hat{\alpha}\right)\right|}{h\left([n \tau]-\left[n \tau_{*}^{\beta}\right]\right)} \geq \delta, \hat{\alpha} \in \mathcal{O}_{\alpha^{*}} . \hat{\beta}_{1} \in \mathcal{O}_{\beta_{1}^{*}}, \hat{\beta}_{2} \in \mathcal{O}_{\beta_{2}^{*}}\right) \\
& +P\left(\hat{\beta}_{1} \in \mathcal{O}_{\beta_{1}^{*}}\right)+P\left(\hat{\beta}_{2} \in \mathcal{O}_{\beta_{2}^{*}}\right)+P\left(\hat{\alpha} \in \mathcal{O}_{\alpha^{*}}\right)
\end{aligned}
$$

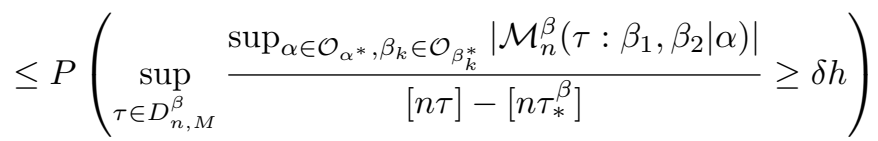

$$
\begin{aligned}
& +P\left(\hat{\beta}_{1} \in \mathcal{O}_{\beta_{1}^{*}}\right)+P\left(\hat{\beta}_{2} \in \mathcal{O}_{\beta_{2}^{*}}\right)+P\left(\hat{\alpha} \in \mathcal{O}_{\alpha^{*}}\right)
\end{aligned}
$$

By the uniform version on the Hájek-Renyi inequality in Lemma 2 of Iacus and Yoshida (2012), we obtain

$$
\begin{aligned}
& P\left(\sup _{\tau \in D_{n, M}^{\beta}} \frac{1}{[n \tau]-\left[n \tau_{*}^{\beta}\right]} \sup _{\substack{\alpha \in \mathcal{O}_{\alpha^{*}}, \beta_{k} \in \mathcal{O}_{\beta_{k}^{*}}}}\left|\mathcal{M}_{n}^{\beta}\left(\tau: \beta_{1}, \beta_{2} \mid \alpha\right)\right| \geq \delta h\right) \\
& \leq P\left(\max _{j>M / h-1} \frac{1}{j} \sup _{\substack{\alpha \in \mathcal{O}_{\alpha^{*}}, \beta_{k} \in \mathcal{O}_{\beta_{k}^{*}}}}\left|\sum_{i=\left[n \tau_{*}^{\beta}\right]+1}^{\left[n \tau_{*}^{\beta}\right]+j}\left(G_{i}\left(\beta_{1} \mid \alpha\right)-G_{i}\left(\beta_{2} \mid \alpha\right)-\mathbb{E}_{\beta_{2}^{*}}\left[G_{i}\left(\beta_{1} \mid \alpha\right)-G_{i}\left(\beta_{2} \mid \alpha\right) \mid \mathscr{G}_{i-1}^{n}\right]\right)\right| \geq \delta h\right) \\
& \leq \sum_{j>M / h-1} \frac{C h}{(\delta h j)^{2}} \\
& \leq \frac{C^{\prime} h}{(\delta h)^{2}} \frac{h}{M}=\frac{C^{\prime}}{\delta^{2} M}=: \gamma_{\beta}(M) .
\end{aligned}
$$

From [C6-II], we have $P\left(\hat{\alpha} \notin \mathcal{O}_{\alpha^{*}}\right)<\epsilon$ and $P\left(\hat{\beta}_{k} \notin \mathcal{O}_{\beta_{k}^{*}}\right)<\epsilon$ for large $n$. Therefore $P_{1, n}^{\beta} \leq \gamma_{\beta}(M)+3 \epsilon$ for large $n$.

[ii] Evaluation of $P_{2, n}^{\beta}$. If $\hat{\alpha} \in \mathcal{O}_{\alpha^{*}}$ and $\hat{\beta}_{k} \in \mathcal{O}_{\beta_{k}^{*}}$, then there exists a positive constant $c$ independent of $i$ such that

$$
\begin{aligned}
\Gamma_{i-1}^{\beta}\left(\hat{\alpha}, \hat{\beta}_{1}, \hat{\beta}_{2}\right) & =\Gamma_{i-1}^{\beta}\left(\alpha^{*}, \beta_{1}^{*}, \beta_{2}^{*}\right)+\left.\int_{0}^{1} \partial_{\left(\alpha, \beta_{1}, \beta_{2}\right)} \Gamma_{i-1}^{\beta}\left(\alpha, \beta_{1}, \beta_{2}\right)\right|_{\substack{\alpha=\alpha^{*}+u\left(\hat{\alpha}-\alpha^{*}\right) \\
\beta_{k}=\beta_{k}^{*}+u\left(\hat{\beta}_{k}-\beta_{k}^{*}\right)}} \mathrm{d} u\left(\begin{array}{c}
\hat{\alpha}-\alpha^{*} \\
\hat{\beta}_{1}-\beta_{1}^{*} \\
\hat{\beta}_{2}-\beta_{2}^{*}
\end{array}\right) \\
& \geq \Gamma_{i-1}^{\beta}\left(\alpha^{*}, \beta_{1}^{*}, \beta_{2}^{*}\right)-c\left(\left|\hat{\alpha}-\alpha^{*}\right|+\left|\hat{\beta}_{1}-\beta_{1}^{*}\right|+\left|\hat{\beta}_{2}-\beta_{2}^{*}\right|\right) .
\end{aligned}
$$

According to $[\mathbf{B} 1-\mathbf{I I}]$, if we set

$$
\delta=\frac{1}{4} \inf _{x} \Gamma^{\beta}\left(x, \alpha^{*}, \beta_{1}^{*}, \beta_{2}^{*}\right)>0
$$

then for large $n$,

$$
\begin{aligned}
P_{2, n}^{\beta} \leq & P\left(\inf _{\tau \in D_{n, M}^{\beta}} \frac{\mathcal{A}_{n}^{\beta}\left(\tau: \hat{\beta}_{1}, \hat{\beta}_{2} \mid \hat{\alpha}\right)}{h\left([n \tau]-\left[n \tau_{*}^{\beta}\right]\right)} \leq 2 \delta, \hat{\alpha} \in \mathcal{O}_{\alpha^{*}}, \hat{\beta}_{1} \in \mathcal{O}_{\beta_{1}^{*}} \hat{\beta}_{2} \in \mathcal{O}_{\beta_{2}^{*}}\right) \\
& +P\left(\hat{\alpha} \notin \mathcal{O}_{\alpha^{*}}\right)+P\left(\hat{\beta}_{1} \notin \mathcal{O}_{\beta_{1}^{*}}\right)+P\left(\hat{\beta}_{2} \notin \mathcal{O}_{\beta_{2}^{*}}\right) \\
\leq & P\left(\inf _{\tau \in D_{n, M}^{\beta}} \frac{1}{[n \tau]-\left[n \tau_{*}^{\beta}\right]} \sum_{i=\left[n \tau_{*}^{\beta}\right]+1}^{[n \tau]}\left(\Gamma_{i-1}^{\beta}\left(\alpha^{*}, \beta_{1}^{*}, \beta_{2}^{*}\right)-c\left(\left|\hat{\alpha}-\alpha^{*}\right|+\left|\hat{\beta}_{1}-\beta_{1}^{*}\right|+\left|\hat{\beta}_{2}-\beta_{2}^{*}\right|\right)\right) \leq 2 \delta\right) \\
& +P\left(\hat{\alpha} \notin \mathcal{O}_{\alpha^{*}}\right)+P\left(\hat{\beta}_{1} \notin \mathcal{O}_{\beta_{1}^{*}}\right)+P\left(\hat{\beta}_{2} \notin \mathcal{O}_{\beta_{2}^{*}}\right)
\end{aligned}
$$




$$
\begin{aligned}
& \leq P\left(\inf _{\tau \in D_{n, M}^{\beta}} \frac{1}{[n \tau]-\left[n \tau_{*}^{\beta}\right]} \sum_{i=\left[n \tau_{*}^{\beta}\right]+1}^{[n \tau]} \Gamma_{i-1}^{\beta}\left(\alpha^{*}, \beta_{1}^{*}, \beta_{2}^{*}\right) \leq 3 \delta\right) \\
& \quad+P\left(-c\left(\left|\hat{\alpha}-\alpha^{*}\right|+\left|\hat{\beta}_{1}-\beta_{1}^{*}\right|+\left|\hat{\beta}_{2}-\beta_{2}^{*}\right|\right) \leq-\delta\right)+P\left(\hat{\alpha} \notin \mathcal{O}_{\alpha^{*}}\right)+P\left(\hat{\beta}_{1} \notin \mathcal{O}_{\beta_{1}^{*}}\right)+P\left(\hat{\beta}_{2} \notin \mathcal{O}_{\beta_{2}^{*}}\right) \\
& \leq P\left(\inf _{x} \Gamma^{\beta}\left(x, \alpha^{*}, \beta_{1}^{*}, \beta_{2}^{*}\right) \leq 3 \delta\right)+P\left(\left|\hat{\alpha}-\alpha^{*}\right|+\left|\hat{\beta}_{1}-\beta_{1}^{*}\right|+\left|\hat{\beta}_{2}-\beta_{2}^{*}\right| \geq \frac{\delta}{c}\right) \\
& \quad+P\left(\hat{\alpha} \notin \mathcal{O}_{\alpha^{*}}\right)+P\left(\hat{\beta}_{1} \notin \mathcal{O}_{\beta_{1}^{*}}\right)+P\left(\hat{\beta}_{2} \notin \mathcal{O}_{\beta_{2}^{*}}\right) \\
& \leq 6 \epsilon
\end{aligned}
$$

thanks to

$$
\begin{aligned}
& P\left(\left|\hat{\alpha}-\alpha^{*}\right|+\left|\hat{\beta}_{1}-\beta_{1}^{*}\right|+\left|\hat{\beta}_{2}-\beta_{2}^{*}\right| \geq \frac{\delta}{c}\right) \\
& \leq P\left(\left|\hat{\alpha}-\alpha^{*}\right| \geq \frac{\delta}{3 c}\right)+P\left(\left|\hat{\beta}_{1}-\beta_{1}^{*}\right| \geq \frac{\delta}{3 c}\right)+P\left(\left|\hat{\beta}_{2}-\beta_{2}^{*}\right| \geq \frac{\delta}{3 c}\right) \\
& \leq 3 \epsilon
\end{aligned}
$$

from [C6-II].

[iii] Evaluation of $P_{3, n}^{\beta}$. We have, for large $n$,

$$
\begin{aligned}
& \operatorname{tr}\left(A_{i-1}^{-1}(\hat{\alpha})\left(b_{i-1}\left(\hat{\beta}_{2}\right)-h \mathbb{E}_{\beta_{2}^{*}}\left[\Delta X_{i} \mid G_{i-1}^{n}\right]\right)\left(b_{i-1}\left(\hat{\beta}_{1}\right)-b_{i-1}\left(\hat{\beta}_{2}\right)\right)^{\top}\right) \\
& =h \operatorname{tr}\left(A_{i-1}^{-1}(\hat{\alpha})\left(b_{i-1}\left(\hat{\beta}_{2}\right)-b_{i-1}\left(\beta_{2}^{*}\right)\right)\left(b_{i-1}\left(\hat{\beta}_{1}\right)-b_{i-1}\left(\hat{\beta}_{2}\right)\right)^{\top}\right)+R_{i-1}\left(h^{2}, \theta\right) \\
& =\left.h \int_{0}^{1} \partial_{\beta} \operatorname{tr}\left(A_{i-1}^{-1}(\hat{\alpha})\left(b_{i-1}(\beta)-b_{i-1}\left(\beta_{2}^{*}\right)\right)\left(b_{i-1}\left(\hat{\beta}_{1}\right)-b_{i-1}\left(\hat{\beta}_{2}\right)\right)^{\top}\right)\right|_{\beta=\beta_{2}^{*}+u\left(\hat{\beta}_{2}-\beta_{2}^{*}\right)} \mathrm{d} u\left(\hat{\beta}_{2}-\beta_{2}^{*}\right)+R_{i-1}\left(h^{2}, \theta\right)
\end{aligned}
$$

and

$$
\begin{aligned}
& \sup _{\tau \in D_{n, M}^{\beta}} \frac{\left|\varrho_{n}^{\beta}\left(\tau: \hat{\beta}_{1}, \hat{\beta}_{2}\right)\right|}{h\left([n \tau]-\left[n \tau_{*}^{\beta}\right]\right)} \\
& \leq \frac{1}{\sqrt{T}} \sup _{\tau \in D_{n, M}^{\beta}} \mid \frac{1}{[n \tau]-\left[n \tau_{*}^{\beta}\right]} \sum_{i=\left[n \tau_{*}^{\beta}\right]+1}^{[n \tau]} \\
& \left.\quad \int_{0}^{1} \partial_{\beta} \operatorname{tr}\left(A_{i-1}^{-1}(\hat{\alpha})\left(b_{i-1}(\beta)-b_{i-1}\left(\beta_{2}^{*}\right)\right)\left(b_{i-1}\left(\hat{\beta}_{1}\right)-b_{i-1}\left(\hat{\beta}_{2}\right)\right)^{\top}\right)\right|_{\beta=\beta_{2}^{*}+u\left(\hat{\beta}_{2}-\beta_{2}^{*}\right)} \mathrm{d} u|\sqrt{T}| \hat{\beta}_{2}-\beta_{2}^{*} \mid \\
& \quad+\sup _{\tau \in D_{n, M}^{\beta}} \frac{h^{2}}{h\left([n \tau]-\left[n \tau_{*}^{\beta}\right]\right)} \sum_{i=\left[n \tau_{*}^{\beta}\right]+1}^{[n \tau]} R_{i-1}(1, \theta) \\
& \leq \frac{1}{\sqrt{T}} \sup _{x, \alpha, \beta_{k}}\left|\left[\operatorname{tr}\left(A^{-1}(x, \alpha) \partial_{\beta^{\ell}} b\left(x, \beta_{1}\right)\left(b\left(x, \beta_{2}\right)-b\left(x, \beta_{3}\right)\right)^{\top}\right)\right]_{\ell}\right| \sqrt{T}\left|\hat{\beta}_{2}-\beta_{2}^{*}\right| \\
& \quad+\frac{h^{2}}{M} \sum_{i=\left[n \tau_{*}^{\beta}\right]+1}^{n} R_{i-1}(1, \theta) \\
& =o_{p}(1) .
\end{aligned}
$$

Hence, we see

$$
\begin{aligned}
P_{3, n}^{\beta} \leq & P\left(\sup _{\tau \in D_{n, M}^{\beta}} \frac{\left|\varrho_{n}^{\beta}\left(\tau: \hat{\beta}_{1}, \hat{\beta}_{2}\right)\right|}{h\left([n \tau]-\left[n \tau_{*}^{\beta}\right]\right)} \geq \delta, \hat{\alpha} \in \mathcal{O}_{\alpha^{*}}, \hat{\beta}_{1} \in \mathcal{O}_{\beta_{1}^{*}}, \hat{\beta}_{2} \in \mathcal{O}_{\beta_{2}^{*}}\right) \\
& \quad+P\left(\hat{\alpha} \notin \mathcal{O}_{\alpha^{*}}\right)+P\left(\hat{\beta}_{1} \notin \mathcal{O}_{\beta_{1}^{*}}\right)+P\left(\hat{\beta}_{2} \notin \mathcal{O}_{\beta_{2}^{*}}\right) \\
\leq & 4 \epsilon
\end{aligned}
$$

for large $n$. 
[iv] From the evaluations in Steps [i]-[iii], we have

$$
\varlimsup_{n \rightarrow \infty} P\left(T\left(\hat{\tau}_{n}^{\beta}-\tau_{*}^{\beta}\right)>M\right) \leq \gamma_{\beta}(M)+13 \epsilon
$$

for any $M \geq 1$ and $\epsilon>0$. Therefore

$$
\varlimsup_{M \rightarrow \infty} \varlimsup_{n \rightarrow \infty} P\left(T\left(\hat{\tau}_{n}^{\beta}-\tau_{*}^{\beta}\right)>M\right) \leq 13 \epsilon
$$

Proof of Proposition 1. We see

$$
\begin{aligned}
\mathcal{T}_{n}^{\alpha}=\frac{1}{\sqrt{2 d n}} \max _{1 \leq k \leq n}\left|\sum_{i=1}^{k} \hat{\eta}_{i}-\frac{k}{n} \sum_{i=1}^{n} \hat{\eta}_{i}\right| & \geq \frac{1}{\sqrt{2 d n}}\left|\sum_{i=1}^{\left[n \tau_{*}^{\alpha}\right]} \hat{\eta}_{i}-\frac{\left[n \tau_{*}^{\alpha}\right]}{n} \sum_{i=1}^{n} \hat{\eta}_{i}\right| \\
& =\sqrt{\frac{n \vartheta_{\alpha}^{2}}{2 d}}\left|\frac{1}{n \vartheta_{\alpha}} \sum_{i=1}^{\left[n \tau_{*}^{\alpha}\right]} \hat{\eta}_{i}-\frac{\left[n \tau_{*}^{\alpha}\right]}{n} \frac{1}{n \vartheta_{\alpha}} \sum_{i=1}^{n} \hat{\eta}_{i}\right| .
\end{aligned}
$$

Now we can express

$$
\begin{aligned}
\hat{\eta}_{i}= & \operatorname{tr}\left(A_{i-1}^{-1}(\hat{\alpha}) \frac{\left(\Delta X_{i}\right)^{\otimes 2}}{h}\right) \\
= & \operatorname{tr}\left(A_{i-1}^{-1}\left(\alpha_{0}\right) \frac{\left(\Delta X_{i}\right)^{\otimes 2}}{h}\right)+\left.\partial_{\alpha} \operatorname{tr}\left(A_{i-1}^{-1}(\alpha) \frac{\left(\Delta X_{i}\right)^{\otimes 2}}{h}\right)\right|_{\alpha=\alpha_{0}}\left(\hat{\alpha}-\alpha_{0}\right) \\
& +\left.\int_{0}^{1}(1-u) \partial_{\alpha}^{2} \operatorname{tr}\left(A_{i-1}^{-1}(\alpha) \frac{\left(\Delta X_{i}\right)^{\otimes 2}}{h}\right)\right|_{\alpha=\alpha_{0}+u\left(\hat{\alpha}-\alpha_{0}\right)} \mathrm{d} u \otimes\left(\hat{\alpha}-\alpha_{0}\right)^{\otimes 2} \\
= & \operatorname{tr}\left(A_{i-1}^{-1}\left(\alpha_{0}\right) \frac{\left(\Delta X_{i}\right)^{\otimes 2}}{h}\right)+\left(\operatorname{tr}\left(A^{-1} \partial_{\alpha^{\ell}} A A_{i-1}^{-1}\left(\alpha_{0}\right) \frac{\left(\Delta X_{i}\right)^{\otimes 2}}{h}\right)\right)_{\ell}\left(\hat{\alpha}-\alpha_{0}\right) \\
& +\left.\int_{0}^{1}(1-u) \partial_{\alpha}^{2} \operatorname{tr}\left(A_{i-1}^{-1}(\alpha) \frac{\left(\Delta X_{i}\right)^{\otimes 2}}{h}\right)\right|_{\alpha=\alpha_{0}+u\left(\hat{\alpha}-\alpha_{0}\right)} \mathrm{d} u \otimes\left(\hat{\alpha}-\alpha_{0}\right)^{\otimes 2} \\
= & : \eta_{1, i}+\eta_{2, i}\left(\hat{\alpha}-\alpha_{0}\right)+\left.\int_{0}^{1}(1-u) \partial_{\alpha}^{2} \operatorname{tr}\left(A_{i-1}^{-1}(\alpha) \frac{\left(\Delta X_{i}\right)^{\otimes 2}}{h}\right)\right|_{\alpha=\alpha_{0}+u\left(\hat{\alpha}-\alpha_{0}\right)} \mathrm{d} u \otimes\left(\hat{\alpha}-\alpha_{0}\right)^{\otimes 2} .
\end{aligned}
$$

Therefore we have, from $[\mathbf{E} 1]$,

$$
\begin{aligned}
& \frac{1}{n \vartheta_{\alpha}} \sum_{i=1}^{\left[n \tau_{*}^{\alpha}\right]} \hat{\eta}_{i}-\frac{\left[n \tau_{*}^{\alpha}\right]}{n} \frac{1}{n \vartheta_{\alpha}} \sum_{i=1}^{n} \hat{\eta}_{i} \\
& =\frac{1}{n \vartheta_{\alpha}} \sum_{i=1}^{\left[n \tau_{*}^{\alpha}\right]} \eta_{1, i}-\frac{\left[n \tau_{*}^{\alpha}\right]}{n} \frac{1}{n \vartheta_{\alpha}} \sum_{i=1}^{n} \eta_{1, i}+\left(\frac{1}{n} \sum_{i=1}^{\left[n \tau_{*}^{\alpha}\right]} \eta_{2, i}-\frac{\left[n \tau_{*}^{\alpha}\right]}{n} \frac{1}{n} \sum_{i=1}^{n} \eta_{2, i}\right) \vartheta_{\alpha}^{-1}\left(\hat{\alpha}-\alpha_{0}\right)+o_{p}(1) \\
& =: \mathcal{H}_{1, n}+\mathcal{H}_{2, n} \vartheta_{\alpha}^{-1}\left(\hat{\alpha}-\alpha_{0}\right)+o_{p}(1) .
\end{aligned}
$$

Here $\mathcal{H}_{1, n}$ and $\mathcal{H}_{2, n}$ can be transformed as follows.

$$
\begin{aligned}
& \mathcal{H}_{1, n}=\left(1-\frac{\left[n \tau_{*}^{\alpha}\right]}{n}\right) \frac{1}{n \vartheta_{\alpha}} \sum_{i=1}^{\left[n \tau_{*}^{\alpha}\right]} \eta_{1, i}-\frac{\left[n \tau_{*}^{\alpha}\right]}{n} \frac{1}{n \vartheta_{\alpha}} \sum_{i=\left[n \tau_{*}^{\alpha}\right]+1}^{n} \eta_{1, i}, \\
& \mathcal{H}_{2, n}=\left(1-\frac{\left[n \tau_{*}^{\alpha}\right]}{n}\right) \frac{1}{n} \sum_{i=1}^{\left[n \tau_{*}^{\alpha}\right]} \eta_{2, i}-\frac{\left[n \tau_{*}^{\alpha}\right]}{n} \frac{1}{n} \sum_{i=\left[n \tau_{*}^{\alpha}\right]+1}^{n} \eta_{2, i} .
\end{aligned}
$$

We have

$$
\left(1-\frac{\left[n \tau_{*}^{\alpha}\right]}{n}\right) \frac{1}{n \vartheta_{\alpha}} \sum_{i=1}^{\left[n \tau_{*}^{\alpha}\right]} \mathbb{E}_{\alpha_{1}^{*}}\left[\eta_{1, i} \mid \mathscr{G}_{i-1}^{n}\right]-\frac{\left[n \tau_{*}^{\alpha}\right]}{n} \frac{1}{n \vartheta_{\alpha}} \sum_{i=\left[n \tau_{*}^{\alpha}\right]+1}^{n} \mathbb{E}_{\alpha_{2}^{*}}\left[\eta_{1, i} \mid \mathscr{G}_{i-1}^{n}\right]
$$




$$
\begin{aligned}
= & \left(1-\frac{\left[n \tau_{*}^{\alpha}\right]}{n}\right) \frac{1}{n \vartheta_{\alpha}} \sum_{i=1}^{\left[n \tau_{*}^{\alpha}\right]} \operatorname{tr}\left(A_{i-1}^{-1}\left(\alpha_{0}\right) A_{i-1}\left(\alpha_{1}^{*}\right)\right) \\
& -\frac{\left[n \tau_{*}^{\alpha}\right]}{n} \frac{1}{n \vartheta_{\alpha}} \sum_{i=\left[n \tau_{*}^{\alpha}\right]+1}^{n} \operatorname{tr}\left(A_{i-1}^{-1}\left(\alpha_{0}\right) A_{i-1}\left(\alpha_{2}^{*}\right)\right)+O_{p}\left(h / \vartheta_{\alpha}\right) \\
= & \left(1-\frac{\left[n \tau_{*}^{\alpha}\right]}{n}\right) \frac{1}{n \vartheta_{\alpha}} \sum_{i=1}^{\left[n \tau_{*}^{\alpha}\right]}\left(d+\left.\partial_{\alpha} \operatorname{tr}\left(A_{i-1}^{-1}\left(\alpha_{0}\right) A_{i-1}(\alpha)\right)\right|_{\alpha=\alpha_{0}}\left(\alpha_{1}^{*}-\alpha_{0}\right)\right. \\
& \left.+\left.\int_{0}^{1}(1-u) \partial_{\alpha}^{2} \operatorname{tr}\left(A_{i-1}^{-1}\left(\alpha_{0}\right) A_{i-1}(\alpha)\right)\right|_{\alpha=\alpha_{0}+u\left(\alpha_{1}^{*}-\alpha_{0}\right)} \otimes\left(\alpha_{1}^{*}-\alpha_{0}\right)^{\otimes 2}\right) \\
& -\frac{\left[n \tau_{*}^{\alpha}\right]}{n} \frac{1}{n \vartheta_{\alpha}} \sum_{i=\left[n \tau_{*}^{\alpha}\right]+1}^{n}\left(d+\left.\partial_{\alpha} \operatorname{tr}\left(A_{i-1}^{-1}\left(\alpha_{0}\right) A_{i-1}(\alpha)\right)\right|_{\alpha=\alpha_{0}}\left(\alpha_{2}^{*}-\alpha_{0}\right)\right. \\
= & \left(1-\frac{\left[n \tau_{*}^{\alpha}\right]}{n}\right) \frac{1}{n} \sum_{i=1}^{\left[n \tau_{*}^{\alpha}\right]}\left(\operatorname{tr}\left(A_{i-1}^{-1}\left(\alpha_{0}\right) \partial_{\alpha^{\ell}} A_{i-1}\left(\alpha_{0}\right)\right)\right)_{\ell} \vartheta_{\alpha}^{-1}\left(\alpha_{1}^{*}-\alpha_{0}\right) \\
& -\frac{\left[n \tau_{*}^{\alpha}\right]}{n} \frac{1}{n} \sum_{i=\left[n \tau_{*}^{\alpha}\right]+1}^{n}\left(\operatorname{tr}\left(A_{i-1}^{-1}\left(\alpha_{0}\right) \partial_{\alpha^{\ell}} A_{i-1}\left(\alpha_{0}\right)\right)\right)_{\ell} \vartheta_{\alpha}^{-1}\left(\alpha_{2}^{*}-\alpha_{0}\right)+o_{p}(1) \\
\stackrel{p}{\longrightarrow} & \tau_{*}^{\alpha}\left(1-\tau_{*}^{\alpha}\right) \int_{\mathbb{R}^{d}}\left(\operatorname{tr}\left(A^{-1} \partial_{\alpha^{\ell}} A\left(x, \alpha_{0}\right)\right)\right)_{\ell} \mathrm{d} \mu_{\alpha_{0}}(x)\left(c_{1}-c_{2}\right)
\end{aligned}
$$

and

$$
\begin{aligned}
& \left(1-\frac{\left[n \tau_{*}^{\alpha}\right]}{n}\right)^{2} \frac{1}{\left(n \vartheta_{\alpha}\right)^{2}} \sum_{i=1}^{\left[n \tau_{*}^{\alpha}\right]} \mathbb{E}_{\alpha_{1}^{*}}\left[\eta_{1, i}^{2} \mid \mathscr{G}_{i-1}^{n}\right]-\left(\frac{\left[n \tau_{*}^{\alpha}\right]}{n}\right)^{2} \frac{1}{\left(n \vartheta_{\alpha}\right)^{2}} \sum_{i=\left[n \tau_{*}^{\alpha}\right]+1}^{n} \mathbb{E}_{\alpha_{2}^{*}}\left[\eta_{1, i}^{2} \mid \mathscr{G}_{i-1}^{n}\right] \\
& =\frac{1}{n \vartheta_{\alpha}^{2}}\left(\left(1-\frac{\left[n \tau_{*}^{\alpha}\right]}{n}\right)^{2} \frac{1}{n} \sum_{i=1}^{\left[n \tau_{*}^{\alpha}\right]} \mathbb{E}_{\alpha_{1}^{*}}\left[\eta_{1, i}^{2} \mid \mathscr{G}_{i-1}^{n}\right]-\left(\frac{\left[n \tau_{*}^{\alpha}\right]}{n}\right)^{2} \frac{1}{n} \sum_{i=\left[n \tau_{*}^{\alpha}\right]+1}^{n} \mathbb{E}_{\alpha_{2}^{*}}\left[\eta_{1, i}^{2} \mid \mathscr{G}_{i-1}^{n}\right]\right) \\
& \stackrel{p}{\longrightarrow} 0 .
\end{aligned}
$$

Therefore, from Lemma 9 of Genon-Catalot and Jacod (1993), we obtain

$$
\mathcal{H}_{1, n} \stackrel{p}{\longrightarrow} \tau_{*}^{\alpha}\left(1-\tau_{*}^{\alpha}\right) \int_{\mathbb{R}^{d}}\left(\operatorname{tr}\left(A^{-1} \partial_{\alpha^{\ell}} A\left(x, \alpha_{0}\right)\right)\right)_{\ell} \mathrm{d} \mu_{\alpha_{0}}(x)\left(c_{1}-c_{2}\right) .
$$

Further, we have

$$
\begin{aligned}
& \left(1-\frac{\left[n \tau_{*}^{\alpha}\right]}{n}\right) \frac{1}{n} \sum_{i=1}^{\left[n \tau_{*}^{\alpha}\right]} \mathbb{E}_{\alpha_{1}^{*}}\left[\eta_{2, i} \mid \mathscr{G}_{i-1}^{n}\right]-\frac{\left[n \tau_{*}^{\alpha}\right]}{n} \frac{1}{n} \sum_{i=\left[n \tau_{*}^{\alpha}\right]+1}^{n} \mathbb{E}_{\alpha_{2}^{*}}\left[\eta_{2, i} \mid \mathscr{G}_{i-1}^{n}\right] \\
& =\left(1-\frac{\left[n \tau_{*}^{\alpha}\right]}{n}\right) \frac{1}{n} \sum_{i=1}^{\left[n \tau_{*}^{\alpha}\right]}\left[\operatorname{tr}\left(A^{-1} \partial_{\alpha^{\ell}} A A_{i-1}^{-1}\left(\alpha_{0}\right) A_{i-1}\left(\alpha_{1}^{*}\right)\right)\right]_{\ell} \\
& \quad-\frac{\left[n \tau_{*}^{\alpha}\right]}{n} \frac{1}{n} \sum_{i=\left[n \tau_{*}^{\alpha}\right]+1}^{n}\left[\operatorname{tr}\left(A^{-1} \partial_{\alpha^{\ell}} A A_{i-1}^{-1}\left(\alpha_{0}\right) A_{i-1}\left(\alpha_{2}^{*}\right)\right)\right]_{\ell}+O_{p}(h) \\
& =\left(1-\frac{\left[n \tau_{*}^{\alpha}\right]}{n}\right) \frac{1}{n} \sum_{i=1}^{\left[n \tau_{*}^{\alpha}\right]}\left(\left[\operatorname{tr}\left(A^{-1} \partial_{\alpha^{\ell}} A_{i-1}\left(\alpha_{0}\right)\right)\right]_{\ell}\right. \\
& \left.\quad+\left.\int_{0}^{1} \partial_{\alpha}\left[\operatorname{tr}\left(A^{-1} \partial_{\alpha^{\ell}} A A_{i-1}^{-1}\left(\alpha_{0}\right) A_{i-1}(\alpha)\right)\right]_{\ell}\right|_{\alpha=\alpha_{0}+u\left(\alpha_{1}^{*}-\alpha_{0}\right)}\left(\alpha_{1}^{*}-\alpha_{0}\right)\right)
\end{aligned}
$$




$$
\begin{aligned}
& \quad-\frac{\left[n \tau_{*}^{\alpha}\right]}{n} \frac{1}{n} \sum_{i=\left[n \tau_{*}^{\alpha}\right]+1}^{n}\left(\left[\operatorname{tr}\left(A^{-1} \partial_{\alpha^{\ell}} A_{i-1}\left(\alpha_{0}\right)\right)\right]_{\ell}\right. \\
& \left.\quad+\left.\int_{0}^{1} \partial_{\alpha}\left[\operatorname{tr}\left(A^{-1} \partial_{\alpha^{\ell}} A A_{i-1}^{-1}\left(\alpha_{0}\right) A_{i-1}(\alpha)\right)\right]_{\ell}\right|_{\alpha=\alpha_{0}+u\left(\alpha_{2}^{*}-\alpha_{0}\right)}\left(\alpha_{2}^{*}-\alpha_{0}\right)\right)+o_{p}(1) \\
& =\left(1-\frac{\left[n \tau_{*}^{\alpha}\right]}{n}\right) \frac{1}{n} \sum_{i=1}^{\left[n \tau_{*}^{\alpha}\right]}\left[\operatorname{tr}\left(A^{-1} \partial_{\alpha^{\ell}} A_{i-1}\left(\alpha_{0}\right)\right)\right]_{\ell}-\frac{\left[n \tau_{*}^{\alpha}\right]}{n} \frac{1}{n} \sum_{i=\left[n \tau_{*}^{\alpha}\right]+1}^{n}\left[\operatorname{tr}\left(A^{-1} \partial_{\alpha^{\ell}} A_{i-1}\left(\alpha_{0}\right)\right)\right]_{\ell}+o_{p}(1) \\
& \stackrel{p}{\longrightarrow} 0
\end{aligned}
$$

and

$$
\begin{aligned}
& \left(1-\frac{\left[n \tau_{*}^{\alpha}\right]}{n}\right)^{2} \frac{1}{n^{2}} \sum_{i=1}^{\left[n \tau_{*}^{\alpha}\right]} \mathbb{E}_{\alpha_{1}^{*}}\left[\eta_{2, i}^{2} \mid \mathscr{G}_{i-1}^{n}\right]-\left(\frac{\left[n \tau_{*}^{\alpha}\right]}{n}\right)^{2} \frac{1}{n^{2}} \sum_{i=\left[n \tau_{*}^{\alpha}\right]+1}^{n} \mathbb{E}_{\alpha_{2}^{*}}\left[\eta_{2, i}^{2} \mid \mathscr{G}_{i-1}^{n}\right] \\
& =\frac{1}{n}\left(\left(1-\frac{\left[n \tau_{*}^{\alpha}\right]}{n}\right)^{2} \frac{1}{n} \sum_{i=1}^{\left[n \tau_{*}^{\alpha}\right]} \mathbb{E}_{\alpha_{1}^{*}}\left[\eta_{2, i}^{2} \mid \mathscr{G}_{i-1}^{n}\right]-\left(\frac{\left[n \tau_{*}^{\alpha}\right]}{n}\right)^{2} \frac{1}{n} \sum_{i=\left[n \tau_{*}^{\alpha}\right]+1}^{n} \mathbb{E}_{\alpha_{2}^{*}}\left[\eta_{2, i}^{2} \mid \mathscr{G}_{i-1}^{n}\right]\right) \\
& \stackrel{p}{\longrightarrow} 0 .
\end{aligned}
$$

Therefore, from Lemma 9 of Genon-Catalot and Jacod (1993), we see $\mathcal{H}_{2, n} \stackrel{p}{\longrightarrow}$ 0. Hence, from 7.52 , 77.53 and this, we obtain

$$
\frac{1}{n \vartheta_{\alpha}} \sum_{i=1}^{\left[n \tau_{*}^{\alpha}\right]} \hat{\eta}_{i}-\frac{\left[n \tau_{*}^{\alpha}\right]}{n} \frac{1}{n \vartheta_{\alpha}} \sum_{i=1}^{n} \hat{\eta}_{i} \stackrel{p}{\longrightarrow} \tau_{*}^{\alpha}\left(1-\tau_{*}^{\alpha}\right) \int_{\mathbb{R}^{d}}\left[\operatorname{tr}\left(A^{-1} \partial_{\alpha^{\ell}} A\left(x, \alpha_{0}\right)\right)\right]_{\ell} \mathrm{d} \mu_{\alpha_{0}}(x)\left(c_{1}-c_{2}\right) \neq 0,
$$

which implies $\mathcal{T}_{n}^{\alpha} \longrightarrow \infty$ from 7.51 and $n \vartheta_{\alpha}^{2} \longrightarrow \infty$, that is, $P\left(\mathcal{T}_{n}^{\alpha}>w_{1}(\epsilon)\right) \longrightarrow 1$.

Proof of Proposition 2, We see

$$
\begin{aligned}
\mathcal{T}_{1, n}^{\beta}=\frac{1}{\sqrt{d T}} \max _{1 \leq k \leq n}\left|\sum_{i=1}^{k} \hat{\xi}_{i}-\frac{k}{n} \sum_{i=1}^{n} \hat{\xi}_{i}\right| & \geq \frac{1}{\sqrt{d T}}\left|\sum_{i=1}^{\left[n \tau_{*}^{\beta}\right]} \hat{\xi}_{i}-\frac{\left[n \tau_{*}^{\beta}\right]}{n} \sum_{i=1}^{n} \hat{\xi}_{i}\right| \\
& =\sqrt{\frac{T \vartheta_{\beta}^{2}}{d}}\left|\frac{1}{T \vartheta_{\beta}} \sum_{i=1}^{\left[n \tau_{*}^{\beta}\right]} \hat{\xi}_{i}-\frac{\left[n \tau_{*}^{\beta}\right]}{n} \frac{1}{T \vartheta_{\beta}} \sum_{i=1}^{n} \hat{\xi}_{i}\right| .
\end{aligned}
$$

Now we can express

$$
\begin{aligned}
\hat{\xi}_{i}= & 1_{d}^{\top} a_{i-1}^{-1}(\hat{\alpha})\left(\Delta X_{i}-h b_{i-1}(\hat{\beta})\right) \\
= & 1_{d}^{\top} a_{i-1}^{-1}\left(\alpha^{*}\right)\left(\Delta X_{i}-h b_{i-1}(\hat{\beta})\right)+\left.\int_{0}^{1} \partial_{\alpha}\left(1_{d}^{\top} a_{i-1}^{-1}(\alpha)\left(\Delta X_{i}-h b_{i-1}(\hat{\beta})\right)\right)\right|_{\alpha=\alpha^{*}+u\left(\hat{\alpha}-\alpha^{*}\right)} \mathrm{d} u\left(\hat{\alpha}-\alpha^{*}\right) \\
= & 1_{d}^{\top} a_{i-1}^{-1}\left(\alpha^{*}\right)\left(\Delta X_{i}-h b_{i-1}\left(\bar{\beta}^{*}\right)\right)-h 1_{d}^{\top} a_{i-1}^{-1}\left(\alpha^{*}\right)\left(b_{i-1}(\hat{\beta})-b_{i-1}\left(\bar{\beta}^{*}\right)\right) \\
& +\left.\int_{0}^{1} \partial_{\alpha}\left(1_{d}^{\top} a_{i-1}^{-1}(\alpha)\left(\Delta X_{i}-h b_{i-1}(\hat{\beta})\right)\right)\right|_{\alpha=\alpha^{*}+u\left(\hat{\alpha}-\alpha^{*}\right)} \mathrm{d} u\left(\hat{\alpha}-\alpha^{*}\right) \\
= & : \xi_{i}-h \int_{0}^{1} 1_{d}^{\top} a_{i-1}^{-1}\left(\alpha^{*}\right) \partial_{\beta} b_{i-1}\left(\bar{\beta}^{*}+u\left(\hat{\beta}-\bar{\beta}^{*}\right)\right) \mathrm{d} u\left(\hat{\beta}-\bar{\beta}^{*}\right) \\
& +\left.\int_{0}^{1} \partial_{\alpha}\left(1_{d}^{\top} a_{i-1}^{-1}(\alpha)\left(\Delta X_{i}-h b_{i-1}(\hat{\beta})\right)\right)\right|_{\alpha=\alpha^{*}+u\left(\hat{\alpha}-\alpha^{*}\right)} \mathrm{d} u\left(\hat{\alpha}-\alpha^{*}\right)
\end{aligned}
$$

Therefore, we have

$$
\frac{1}{T \vartheta_{\beta}} \sum_{i=1}^{\left[n \tau_{*}^{\beta}\right]} \hat{\xi}_{i}-\frac{\left[n \tau_{*}^{\beta}\right]}{n} \frac{1}{T \vartheta_{\beta}} \sum_{i=1}^{n} \hat{\xi}_{i}=\frac{1}{T \vartheta_{\beta}} \sum_{i=1}^{\left[n \tau_{*}^{\beta}\right]} \xi_{i}-\frac{\left[n \tau_{*}^{\beta}\right]}{n} \frac{1}{T \vartheta_{\beta}} \sum_{i=1}^{n} \xi_{i}+o_{p}(1)
$$




$$
\begin{aligned}
& =\left(1-\frac{\left[n \tau_{*}^{\beta}\right]}{n}\right) \frac{1}{T \vartheta_{\beta}} \sum_{i=1}^{\left[n \tau_{*}^{\beta}\right]} \xi_{i}-\frac{\left[n \tau_{*}^{\beta}\right]}{n} \frac{1}{T \vartheta_{\beta}} \sum_{i=\left[n \tau_{*}^{\beta}\right]+1}^{n} \xi_{i}+o_{p}(1), \\
& \left(1-\frac{\left[n \tau_{*}^{\beta}\right]}{n}\right) \frac{1}{T \vartheta_{\beta}} \sum_{i=1}^{\left[n \tau_{*}^{\beta}\right]} \mathbb{E}_{\beta_{1}^{*}}\left[\xi_{i} \mid \mathscr{G}_{i-1}^{n}\right]-\frac{\left[n \tau_{*}^{\beta}\right]}{n} \frac{1}{T \vartheta_{\beta}} \sum_{i=\left[n \tau_{*}^{\beta}\right]+1}^{n} \mathbb{E}_{\beta_{2}^{*}}\left[\xi_{i} \mid \mathscr{G}_{i-1}^{n}\right] \\
& =\left(1-\frac{\left[n \tau_{*}^{\beta}\right]}{n}\right) \frac{1}{T \vartheta_{\beta}} \sum_{i=1}^{\left[n \tau_{*}^{\beta}\right]} h 1_{d}^{\top} a_{i-1}^{-1}\left(\alpha^{*}\right)\left(b_{i-1}\left(\beta_{1}^{*}\right)-b_{i-1}\left(\bar{\beta}^{*}\right)\right) \\
& -\frac{\left[n \tau_{*}^{\beta}\right]}{n} \frac{1}{T \vartheta_{\beta}} \sum_{i=\left[n \tau_{*}^{\beta}\right]+1}^{n} h 1_{d}^{\top} a_{i-1}^{-1}\left(\alpha^{*}\right)\left(b_{i-1}\left(\beta_{2}^{*}\right)-b_{i-1}\left(\bar{\beta}^{*}\right)\right)+O_{p}\left(h / \vartheta_{\beta}\right) \\
& =\left(1-\frac{\left[n \tau_{*}^{\beta}\right]}{n}\right) \frac{1}{n \vartheta_{\beta}} \sum_{i=1}^{\left[n \tau_{*}^{\beta}\right]} 1_{d}^{\top} a_{i-1}^{-1}\left(\alpha^{*}\right)\left(b_{i-1}\left(\beta_{0}\right)-b_{i-1}\left(\bar{\beta}^{*}\right)\right) \\
& -\frac{\left[n \tau_{*}^{\beta}\right]}{n} \frac{1}{n \vartheta_{\beta}} \sum_{i=\left[n \tau_{*}^{\beta}\right]+1}^{n} 1_{d}^{\top} a_{i-1}^{-1}\left(\alpha^{*}\right)\left(b_{i-1}\left(\beta_{0}\right)-b_{i-1}\left(\bar{\beta}^{*}\right)\right) \\
& +\left(1-\frac{\left[n \tau_{*}^{\beta}\right]}{n}\right) \frac{1}{n \vartheta_{\beta}} \sum_{i=1}^{\left[n \tau_{*}^{\beta}\right]} 1_{d}^{\top} a_{i-1}^{-1}\left(\alpha^{*}\right) \partial_{\beta} b_{i-1}\left(\beta_{0}\right)\left(\beta_{1}^{*}-\beta_{0}\right) \\
& -\frac{\left[n \tau_{*}^{\beta}\right]}{n} \frac{1}{n \vartheta_{\beta}} \sum_{i=\left[n \tau_{*}^{\beta}\right]+1}^{n} 1_{d}^{\top} a_{i-1}^{-1}\left(\alpha^{*}\right) \partial_{\beta} b_{i-1}\left(\beta_{0}\right)\left(\beta_{2}^{*}-\beta_{0}\right) \\
& +\left(1-\frac{\left[n \tau_{*}^{\beta}\right]}{n}\right) \frac{1}{n \vartheta_{\beta}} \sum_{i=1}^{\left[n \tau_{*}^{\beta}\right]} 1_{d}^{\top} a_{i-1}^{-1}\left(\alpha^{*}\right) \int_{0}^{1}(1-u) \partial_{\beta}^{2} b_{i-1}\left(\beta_{0}+u\left(\beta_{1}^{*}-\beta_{0}\right)\right) \mathrm{d} u \otimes\left(\beta_{1}^{*}-\beta_{0}\right)^{\otimes 2} \\
& -\frac{\left[n \tau_{*}^{\beta}\right]}{n} \frac{1}{n \vartheta_{\beta}} \sum_{i=\left[n \tau_{*}^{\beta}\right]+1}^{n} 1_{d}^{\top} a_{i-1}^{-1}\left(\alpha^{*}\right) \int_{0}^{1}(1-u) \partial_{\beta}^{2} b_{i-1}\left(\beta_{0}+u\left(\beta_{2}^{*}-\beta_{0}\right)\right) \mathrm{d} u \otimes\left(\beta_{2}^{*}-\beta_{0}\right)^{\otimes 2}+o_{p}(1) \\
& =-\left(1-\frac{\left[n \tau_{*}^{\beta}\right]}{n}\right) \frac{1}{n \vartheta_{\beta}} \sum_{i=1}^{\left[n \tau_{*}^{\beta}\right]} 1_{d}^{\top} a_{i-1}^{-1}\left(\alpha^{*}\right)\left(\partial_{\beta} b_{i-1}\left(\beta_{0}\right)\left(\bar{\beta}^{*}-\beta_{0}\right)\right. \\
& \left.+\int_{0}^{1}(1-u) \partial_{\beta}^{2} b_{i-1}\left(\beta_{0}+u\left(\bar{\beta}^{*}-\beta_{0}\right)\right) \mathrm{d} u \otimes\left(\bar{\beta}^{*}-\beta_{0}\right)^{\otimes 2}\right) \\
& +\frac{\left[n \tau_{*}^{\beta}\right]}{n} \frac{1}{n \vartheta_{\beta}} \sum_{i=\left[n \tau_{*}^{\beta}\right]+1}^{n} 1_{d}^{\top} a_{i-1}^{-1}\left(\alpha^{*}\right)\left(\partial_{\beta} b_{i-1}\left(\beta_{0}\right)\left(\bar{\beta}^{*}-\beta_{0}\right)\right. \\
& \left.+\int_{0}^{1}(1-u) \partial_{\beta}^{2} b_{i-1}\left(\beta_{0}+u\left(\bar{\beta}^{*}-\beta_{0}\right)\right) \mathrm{d} u \otimes\left(\bar{\beta}^{*}-\beta_{0}\right)^{\otimes 2}\right) \\
& +\left(1-\frac{\left[n \tau_{*}^{\beta}\right]}{n}\right) \frac{1}{n} \sum_{i=1}^{\left[n \tau_{*}^{\beta}\right]} 1_{d}^{\top} a_{i-1}^{-1}\left(\alpha^{*}\right) \partial_{\beta} b_{i-1}\left(\beta_{0}\right) \vartheta_{\beta}^{-1}\left(\beta_{1}^{*}-\beta_{0}\right) \\
& -\frac{\left[n \tau_{*}^{\beta}\right]}{n} \frac{1}{n} \sum_{i=\left[n \tau_{*}^{\beta}\right]+1}^{n} 1_{d}^{\top} a_{i-1}^{-1}\left(\alpha^{*}\right) \partial_{\beta} b_{i-1}\left(\beta_{0}\right) \vartheta_{\beta}^{-1}\left(\beta_{2}^{*}-\beta_{0}\right)+o_{p}(1) \\
& =-\left(1-\frac{\left[n \tau_{*}^{\beta}\right]}{n}\right) \frac{1}{n} \sum_{i=1}^{\left[n \tau_{*}^{\beta}\right]} 1_{d}^{\top} a_{i-1}^{-1}\left(\alpha^{*}\right) \partial_{\beta} b_{i-1}\left(\beta_{0}\right) \vartheta_{\beta}^{-1}\left(\bar{\beta}^{*}-\beta_{0}\right)
\end{aligned}
$$




$$
\begin{aligned}
&+ \frac{\left[n \tau_{*}^{\beta}\right]}{n} \frac{1}{n} \sum_{i=\left[n \tau_{*}^{\beta}\right]+1}^{n} 1_{d}^{\top} a_{i-1}^{-1}\left(\alpha^{*}\right) \partial_{\beta} b_{i-1}\left(\beta_{0}\right) \vartheta_{\beta}^{-1}\left(\bar{\beta}^{*}-\beta_{0}\right) \\
&+\left(1-\frac{\left[n \tau_{*}^{\beta}\right]}{n}\right) \frac{1}{n} \sum_{i=1}^{\left[n \tau_{*}^{\beta}\right]} 1_{d}^{\top} a_{i-1}^{-1}\left(\alpha^{*}\right) \partial_{\beta} b_{i-1}\left(\beta_{0}\right) \vartheta_{\beta}^{-1}\left(\beta_{1}^{*}-\beta_{0}\right) \\
&-\frac{\left[n \tau_{*}^{\beta}\right]}{n} \frac{1}{n} \sum_{i=\left[n \tau_{*}^{\beta}\right]+1}^{n} 1_{d}^{\top} a_{i-1}^{-1}\left(\alpha^{*}\right) \partial_{\beta} b_{i-1}\left(\beta_{0}\right) \vartheta_{\beta}^{-1}\left(\beta_{2}^{*}-\beta_{0}\right)+o_{p}(1) \\
& \stackrel{p}{\longrightarrow} \tau_{*}^{\beta}\left(1-\tau_{*}^{\beta}\right) \int_{\mathbb{R}^{d}} 1_{d}^{\top} a^{-1}\left(x, \alpha^{*}\right) \partial_{\beta} b\left(x, \beta_{0}\right) \mathrm{d} \mu_{\left(\alpha^{*}, \beta_{0}\right)}(x)\left(d_{1}-d_{2}\right),
\end{aligned}
$$

and

$$
\begin{aligned}
& \left(1-\frac{\left[n \tau_{*}^{\beta}\right]}{n}\right)^{2} \frac{1}{\left(T \vartheta_{\beta}\right)^{2}} \sum_{i=1}^{\left[n \tau_{*}^{\beta}\right]} \mathbb{E}_{\beta_{1}^{*}}\left[\xi_{i}^{2} \mid \mathscr{G}_{i-1}^{n}\right]-\left(\frac{\left[n \tau_{*}^{\beta}\right]}{n}\right)^{2} \frac{1}{\left(T \vartheta_{\beta}\right)^{2}} \sum_{i=\left[n \tau^{*}\right]+1}^{n} \mathbb{E}_{\beta_{2}^{*}}\left[\xi_{i}^{2} \mid \mathscr{G}_{i-1}^{n}\right] \\
& =\frac{1}{T \vartheta_{\beta}^{2}}\left(\left(1-\frac{\left[n \tau_{*}^{\beta}\right]}{n}\right)^{2} \frac{1}{T} \sum_{i=1}^{\left[n \tau_{*}^{\beta}\right]} \mathbb{E}_{\beta_{1}^{*}}\left[\xi_{i}^{2} \mid \mathscr{G}_{i-1}^{n}\right]-\left(\frac{\left[n \tau_{*}^{\beta}\right]}{n}\right)^{2} \frac{1}{T} \sum_{i=\left[n \tau^{*}\right]+1}^{n} \mathbb{E}_{\beta_{2}^{*}}\left[\xi_{i}^{2} \mid \mathscr{G}_{i-1}^{n}\right]\right) \\
& \stackrel{p}{\longrightarrow} 0 .
\end{aligned}
$$

Therefore, from Lemma 9 of Genon-Catalot and Jacod (1993), we see

$$
\frac{1}{T \vartheta_{\beta}} \sum_{i=1}^{\left[n \tau_{*}^{\beta}\right]} \xi_{i}-\frac{\left[n \tau_{*}^{\beta}\right]}{n} \frac{1}{T \vartheta_{\beta}} \sum_{i=1}^{n} \xi_{i} \stackrel{p}{\longrightarrow} \tau_{*}^{\beta}\left(1-\tau_{*}^{\beta}\right) \int_{\mathbb{R}^{d}} 1_{d}^{\top} a^{-1}\left(x, \alpha^{*}\right) \partial_{\beta} b\left(x, \beta_{0}\right) \mathrm{d} \mu_{\beta_{0}}(x)\left(d_{1}-d_{2}\right) .
$$

Hence, from 7.55 and this, we obtain

$$
\frac{1}{T \vartheta_{\beta}} \sum_{i=1}^{\left[n \tau_{*}^{\beta}\right]} \hat{\xi}_{i}-\frac{\left[n \tau_{*}^{\beta}\right]}{n} \frac{1}{T \vartheta_{\beta}} \sum_{i=1}^{n} \hat{\xi}_{i} \stackrel{p}{\longrightarrow} \tau_{*}^{\beta}\left(1-\tau_{*}^{\beta}\right) \int_{\mathbb{R}^{d}} 1_{d}^{\top} a^{-1}\left(x, \alpha^{*}\right) \partial_{\beta} b\left(x, \beta_{0}\right) \mathrm{d} \mu_{\beta_{0}}(x)\left(d_{1}-d_{2}\right) \neq 0,
$$

which implies $\mathcal{T}_{1, n}^{\beta} \longrightarrow \infty$ from $\left(7.54\right.$ and $T \vartheta_{\beta}^{2} \longrightarrow \infty$, that is, $P\left(\mathcal{T}_{1, n}^{\beta}>w_{1}(\epsilon)\right) \longrightarrow 1$.

Proof of Proposition 3 . We see

$$
\begin{aligned}
\mathcal{T}_{2, n}^{\beta} & =\frac{1}{\sqrt{T}} \max _{1 \leq k \leq n}\left\|\mathcal{I}_{n}^{-1 / 2}\left(\sum_{i=1}^{k} \hat{\zeta}_{i}-\frac{k}{n} \sum_{i=1}^{n} \hat{\zeta}_{i}\right)\right\| \\
& \geq \frac{1}{\sqrt{T}}\left\|\mathcal{I}_{n}^{-1 / 2}\left(\sum_{i=1}^{\left[n \tau_{*}^{\beta}\right]} \hat{\zeta}_{i}-\frac{\left[n \tau_{*}^{\beta}\right]}{n} \sum_{i=1}^{n} \hat{\zeta}_{i}\right)\right\| \\
& =\sqrt{T \vartheta_{\beta}^{2}}\left\|\mathcal{I}_{n}^{-1 / 2}\left(\frac{1}{T \vartheta_{\beta}} \sum_{i=1}^{\left[n \tau_{*}^{\beta}\right]} \hat{\zeta}_{i}-\frac{\left[n \tau_{*}^{\beta}\right]}{n} \frac{1}{T \vartheta_{\beta}} \sum_{i=1}^{n} \hat{\zeta}_{i}\right)\right\| .
\end{aligned}
$$

Now we can express

$$
\begin{aligned}
\hat{\zeta}_{i}= & \partial_{\beta} b_{i-1}(\hat{\beta})^{\top} A_{i-1}^{-1}(\hat{\alpha})\left(\Delta X_{i}-h b_{i-1}(\hat{\beta})\right) \\
= & \partial_{\beta} b_{i-1}(\hat{\beta})^{\top} A_{i-1}^{-1}\left(\alpha^{*}\right)\left(\Delta X_{i}-h b_{i-1}(\hat{\beta})\right) \\
& \quad+\left.\int_{0}^{1} \partial_{\alpha}\left(\partial_{\beta} b_{i-1}(\hat{\beta})^{\top} A_{i-1}^{-1}(\alpha)\left(\Delta X_{i}-h b_{i-1}(\hat{\beta})\right)\right)\right|_{\alpha=\alpha^{*}+u\left(\hat{\alpha}-\alpha^{*}\right)} \mathrm{d} u\left(\hat{\alpha}-\alpha^{*}\right) \\
= & \partial_{\beta} b_{i-1}(\hat{\beta})^{\top} A_{i-1}^{-1}\left(\alpha^{*}\right)\left(\Delta X_{i}-h b_{i-1}\left(\bar{\beta}^{*}\right)\right)-h \partial_{\beta} b_{i-1}(\hat{\beta})^{\top} A_{i-1}^{-1}\left(\alpha^{*}\right)\left(b_{i-1}(\hat{\beta})-b_{i-1}\left(\bar{\beta}^{*}\right)\right) \\
& \quad+\left.\int_{0}^{1} \partial_{\alpha}\left(\partial_{\beta} b_{i-1}(\hat{\beta})^{\top} A_{i-1}^{-1}(\alpha)\left(\Delta X_{i}-h b_{i-1}(\hat{\beta})\right)\right)\right|_{\alpha=\alpha^{*}+u\left(\hat{\alpha}-\alpha^{*}\right)} \mathrm{d} u\left(\hat{\alpha}-\alpha^{*}\right) \\
& =\partial_{\beta} b_{i-1}\left(\beta^{*}\right)^{\top} A_{i-1}^{-1}\left(\alpha^{*}\right)\left(\Delta X_{i}-h b_{i-1}\left(\bar{\beta}^{*}\right)\right)
\end{aligned}
$$




$$
\begin{aligned}
& +\left.\sum_{j=1}^{M-1} \partial_{\beta}^{j}\left(\partial_{\beta} b_{i-1}(\beta)^{\top} A_{i-1}^{-1}\left(\alpha^{*}\right)\left(\Delta X_{i}-h b_{i-1}\left(\bar{\beta}^{*}\right)\right)\right)\right|_{\beta=\bar{\beta}^{*}} \otimes\left(\hat{\beta}-\bar{\beta}^{*}\right)^{\otimes j} \\
& +\left.\int_{0}^{1} \partial_{\beta}^{M}\left(\partial_{\beta} b_{i-1}(\beta)^{\top} A_{i-1}^{-1}\left(\alpha^{*}\right)\left(\Delta X_{i}-h b_{i-1}\left(\bar{\beta}^{*}\right)\right)\right)\right|_{\beta=\bar{\beta}^{*}+u\left(\hat{\beta}-\bar{\beta}^{*}\right)} \otimes\left(\hat{\beta}-\bar{\beta}^{*}\right)^{\otimes M} \\
& -h \int_{0}^{1} \partial_{\beta} b_{i-1}(\hat{\beta})^{\top} A_{i-1}^{-1}\left(\alpha^{*}\right) \partial_{\beta} b_{i-1}\left(\bar{\beta}^{*}+u\left(\hat{\beta}-\bar{\beta}^{*}\right)\right) \mathrm{d} u\left(\hat{\beta}-\bar{\beta}^{*}\right) \\
& +\left.\int_{0}^{1} \partial_{\alpha}\left(\partial_{\beta} b_{i-1}(\hat{\beta})^{\top} A_{i-1}^{-1}(\alpha)\left(\Delta X_{i}-h b_{i-1}(\hat{\beta})\right)\right)\right|_{\alpha=\alpha^{*}+u\left(\hat{\alpha}-\alpha^{*}\right)} \mathrm{d} u\left(\hat{\alpha}-\alpha^{*}\right) \\
=: & \zeta_{i}+\left.\sum_{j=1}^{M-1} \partial_{\beta}^{j}\left(\partial_{\beta} b_{i-1}(\beta)^{\top} A_{i-1}^{-1}\left(\alpha^{*}\right)\left(\Delta X_{i}-h b_{i-1}\left(\bar{\beta}^{*}\right)\right)\right)\right|_{\beta=\bar{\beta}^{*}} \otimes\left(\hat{\beta}-\bar{\beta}^{*}\right)^{\otimes j} \\
& +\left.\int_{0}^{1} \partial_{\beta}^{M}\left(\partial_{\beta} b_{i-1}(\beta)^{\top} A_{i-1}^{-1}\left(\alpha^{*}\right)\left(\Delta X_{i}-h b_{i-1}\left(\bar{\beta}^{*}\right)\right)\right)\right|_{\beta=\bar{\beta}^{*}+u\left(\hat{\beta}-\bar{\beta}^{*}\right)} \otimes\left(\hat{\beta}-\bar{\beta}^{*}\right)^{\otimes M} \\
& -h \int_{0}^{1} \partial_{\beta} b_{i-1}(\hat{\beta})^{\top} A_{i-1}^{-1}\left(\alpha^{*}\right) \partial_{\beta} b_{i-1}\left(\bar{\beta}^{*}+u\left(\hat{\beta}-\bar{\beta}^{*}\right)\right) \mathrm{d} u\left(\hat{\beta}-\bar{\beta}^{*}\right) \\
& +\left.\int_{0}^{1} \partial_{\alpha}\left(\partial_{\beta} b_{i-1}(\hat{\beta})^{\top} A_{i-1}^{-1}(\alpha)\left(\Delta X_{i}-h b_{i-1}(\hat{\beta})\right)\right)\right|_{\alpha=\alpha^{*}+u\left(\hat{\alpha}-\alpha^{*}\right)} \mathrm{d} u\left(\hat{\alpha}-\alpha^{*}\right) .
\end{aligned}
$$

Thus, we have

$$
\begin{aligned}
& \frac{1}{T \vartheta_{\beta}} \sum_{i=1}^{\left[n \tau_{*}^{\beta}\right]} \hat{\zeta}_{i}-\frac{\left[n \tau_{*}^{\beta}\right]}{n} \frac{1}{T \vartheta_{\beta}} \sum_{i=1}^{n} \hat{\zeta}_{i}=\frac{1}{T \vartheta_{\beta}} \sum_{i=1}^{\left[n \tau_{*}^{\beta}\right]} \zeta_{i}-\frac{\left[n \tau_{*}^{\beta}\right]}{n} \frac{1}{T \vartheta_{\beta}} \sum_{i=1}^{n} \zeta_{i}+o_{p}(1) \\
& =\left(1-\frac{\left[n \tau_{*}^{\beta}\right]}{n}\right) \frac{1}{T \vartheta_{\beta}} \sum_{i=1}^{\left[n \tau_{*}^{\beta}\right]} \zeta_{i}-\frac{\left[n \tau_{*}^{\beta}\right]}{n} \frac{1}{T \vartheta_{\beta}} \sum_{i=\left[n \tau_{*}^{\beta}\right]+1}^{n} \zeta_{i}+o_{p}(1), \\
& \left(1-\frac{\left[n \tau_{*}^{\beta}\right]}{n}\right) \frac{1}{T \vartheta_{\beta}} \sum_{i=1}^{\left[n \tau_{*}^{\beta}\right]} \mathbb{E}_{\beta_{1}^{*}}\left[\zeta_{i} \mid \mathscr{G}_{i-1}^{n}\right]-\frac{\left[n \tau_{*}^{\beta}\right]}{n} \frac{1}{T \vartheta_{\beta}} \sum_{i=\left[n \tau_{*}^{\beta}\right]+1}^{n} \mathbb{E}_{\beta_{2}^{*}}\left[\zeta_{i} \mid \mathscr{G}_{i-1}^{n}\right] \\
& =\left(1-\frac{\left[n \tau_{*}^{\beta}\right]}{n}\right) \frac{1}{T \vartheta_{\beta}} \sum_{i=1}^{\left[n \tau_{*}^{\beta}\right]} h \partial_{\beta} b_{i-1}\left(\bar{\beta}^{*}\right)^{\top} A_{i-1}^{-1}\left(\alpha^{*}\right)\left(b_{i-1}\left(\beta_{1}^{*}\right)-b_{i-1}\left(\bar{\beta}^{*}\right)\right) \\
& -\frac{\left[n \tau_{*}^{\beta}\right]}{n} \frac{1}{T \vartheta_{\beta}} \sum_{i=\left[n \tau_{*}^{\beta}\right]+1}^{n} h \partial_{\beta} b_{i-1}\left(\bar{\beta}^{*}\right)^{\top} A_{i-1}^{-1}\left(\alpha^{*}\right)\left(b_{i-1}\left(\beta_{2}^{*}\right)-b_{i-1}\left(\bar{\beta}^{*}\right)\right)+O_{p}\left(h / \vartheta_{\beta}\right) \\
& =\left(1-\frac{\left[n \tau_{*}^{\beta}\right]}{n}\right) \frac{1}{n \vartheta_{\beta}} \sum_{i=1}^{\left[n \tau_{*}^{\beta}\right]} \partial_{\beta} b_{i-1}\left(\bar{\beta}^{*}\right)^{\top} A_{i-1}^{-1}\left(\alpha^{*}\right)\left(b_{i-1}\left(\beta_{0}\right)-b_{i-1}\left(\bar{\beta}^{*}\right)\right) \\
& -\frac{\left[n \tau_{*}^{\beta}\right]}{n} \frac{1}{n \vartheta_{\beta}} \sum_{i=\left[n \tau_{*}^{\beta}\right]+1}^{n} \partial_{\beta} b_{i-1}\left(\bar{\beta}^{*}\right)^{\top} A_{i-1}^{-1}\left(\alpha^{*}\right)\left(b_{i-1}\left(\beta_{0}\right)-b_{i-1}\left(\bar{\beta}^{*}\right)\right) \\
& +\left(1-\frac{\left[n \tau_{*}^{\beta}\right]}{n}\right) \frac{1}{n \vartheta_{\beta}} \sum_{i=1}^{\left[n \tau_{*}^{\beta}\right]} \partial_{\beta} b_{i-1}\left(\bar{\beta}^{*}\right)^{\top} A_{i-1}^{-1}\left(\alpha^{*}\right) \partial_{\beta} b_{i-1}\left(\beta_{0}\right)\left(\beta_{1}^{*}-\beta_{0}\right) \\
& -\frac{\left[n \tau_{*}^{\beta}\right]}{n} \frac{1}{n \vartheta_{\beta}} \sum_{i=\left[n \tau_{*}^{\beta}\right]+1}^{n} \partial_{\beta} b_{i-1}\left(\bar{\beta}^{*}\right)^{\top} A_{i-1}^{-1}\left(\alpha^{*}\right) \partial_{\beta} b_{i-1}\left(\beta_{0}\right)\left(\beta_{2}^{*}-\beta_{0}\right) \\
& +\left(1-\frac{\left[n \tau_{*}^{\beta}\right]}{n}\right) \frac{1}{n \vartheta_{\beta}} \sum_{i=1}^{\left[n \tau_{*}^{\beta}\right]} \partial_{\beta} b_{i-1}\left(\bar{\beta}^{*}\right)^{\top} A_{i-1}^{-1}\left(\alpha^{*}\right) \int_{0}^{1}(1-u) \partial_{\beta}^{2} b_{i-1}\left(\beta_{0}+u\left(\beta_{1}^{*}-\beta_{0}\right)\right) \mathrm{d} u \otimes\left(\beta_{1}^{*}-\beta_{0}\right)^{\otimes 2}
\end{aligned}
$$




$$
\begin{aligned}
& -\frac{\left[n \tau_{*}^{\beta}\right]}{n} \frac{1}{n \vartheta_{\beta}} \sum_{i=\left[n \tau_{*}^{\beta}\right]+1}^{n} \partial_{\beta} b_{i-1}\left(\bar{\beta}^{*}\right)^{\top} A_{i-1}^{-1}\left(\alpha^{*}\right) \int_{0}^{1}(1-u) \partial_{\beta}^{2} b_{i-1}\left(\beta_{0}+u\left(\beta_{2}^{*}-\beta_{0}\right)\right) \mathrm{d} u \otimes\left(\beta_{2}^{*}-\beta_{0}\right)^{\otimes 2}+o_{p}(1) \\
& =-\left(1-\frac{\left[n \tau_{*}^{\beta}\right]}{n}\right) \frac{1}{n \vartheta_{\beta}} \sum_{i=1}^{\left[n \tau_{*}^{\beta}\right]} \partial_{\beta} b_{i-1}\left(\bar{\beta}^{*}\right)^{\top} A_{i-1}^{-1}\left(\alpha^{*}\right)\left(\partial_{\beta} b_{i-1}\left(\beta_{0}\right)\left(\bar{\beta}^{*}-\beta_{0}\right)\right. \\
& \left.+\int_{0}^{1}(1-u) \partial_{\beta}^{2} b_{i-1}\left(\beta_{0}+u\left(\bar{\beta}^{*}-\beta_{0}\right)\right) \mathrm{d} u \otimes\left(\bar{\beta}^{*}-\beta_{0}\right)^{\otimes 2}\right) \\
& +\frac{\left[n \tau_{*}^{\beta}\right]}{n} \frac{1}{n \vartheta_{\beta}} \sum_{i=\left[n \tau_{*}^{\beta}\right]+1}^{n} \partial_{\beta} b_{i-1}\left(\bar{\beta}^{*}\right)^{\top} A_{i-1}^{-1}\left(\alpha^{*}\right)\left(\partial_{\beta} b_{i-1}\left(\beta_{0}\right)\left(\bar{\beta}^{*}-\beta_{0}\right)\right. \\
& \left.+\int_{0}^{1}(1-u) \partial_{\beta}^{2} b_{i-1}\left(\beta_{0}+u\left(\bar{\beta}^{*}-\beta_{0}\right)\right) \mathrm{d} u \otimes\left(\bar{\beta}^{*}-\beta_{0}\right)^{\otimes 2}\right) \\
& +\left(1-\frac{\left[n \tau_{*}^{\beta}\right]}{n}\right) \frac{1}{n} \sum_{i=1}^{\left[n \tau_{*}^{\beta}\right]} \partial_{\beta} b_{i-1}\left(\bar{\beta}^{*}\right)^{\top} A_{i-1}^{-1}\left(\alpha^{*}\right) \partial_{\beta} b_{i-1}\left(\beta_{0}\right) \vartheta_{\beta}^{-1}\left(\beta_{1}^{*}-\beta_{0}\right) \\
& -\frac{\left[n \tau_{*}^{\beta}\right]}{n} \frac{1}{n} \sum_{i=\left[n \tau_{*}^{\beta}\right]+1}^{n} \partial_{\beta} b_{i-1}\left(\bar{\beta}^{*}\right)^{\top} A_{i-1}^{-1}\left(\alpha^{*}\right) \partial_{\beta} b_{i-1}\left(\beta_{0}\right) \vartheta_{\beta}^{-1}\left(\beta_{2}^{*}-\beta_{0}\right)+o_{p}(1) \\
& =-\left(1-\frac{\left[n \tau_{*}^{\beta}\right]}{n}\right) \frac{1}{n} \sum_{i=1}^{\left[n \tau_{*}^{\beta}\right]} \partial_{\beta} b_{i-1}\left(\bar{\beta}^{*}\right)^{\top} A_{i-1}^{-1}\left(\alpha^{*}\right) \partial_{\beta} b_{i-1}\left(\beta_{0}\right) \vartheta_{\beta}^{-1}\left(\bar{\beta}^{*}-\beta_{0}\right) \\
& +\frac{\left[n \tau_{*}^{\beta}\right]}{n} \frac{1}{n} \sum_{i=\left[n \tau_{*}^{\beta}\right]+1}^{n} \partial_{\beta} b_{i-1}\left(\bar{\beta}^{*}\right)^{\top} A_{i-1}^{-1}\left(\alpha^{*}\right) \partial_{\beta} b_{i-1}\left(\beta_{0}\right) \vartheta_{\beta}^{-1}\left(\bar{\beta}^{*}-\beta_{0}\right) \\
& +\left(1-\frac{\left[n \tau_{*}^{\beta}\right]}{n}\right) \frac{1}{n} \sum_{i=1}^{\left[n \tau_{*}^{\beta}\right]} \partial_{\beta} b_{i-1}\left(\bar{\beta}^{*}\right)^{\top} A_{i-1}^{-1}\left(\alpha^{*}\right) \partial_{\beta} b_{i-1}\left(\beta_{0}\right) \vartheta_{\beta}^{-1}\left(\beta_{1}^{*}-\beta_{0}\right) \\
& -\frac{\left[n \tau_{*}^{\beta}\right]}{n} \frac{1}{n} \sum_{i=\left[n \tau_{*}^{\beta}\right]+1}^{n} \partial_{\beta} b_{i-1}\left(\bar{\beta}^{*}\right)^{\top} A_{i-1}^{-1}\left(\alpha^{*}\right) \partial_{\beta} b_{i-1}\left(\beta_{0}\right) \vartheta_{\beta}^{-1}\left(\beta_{2}^{*}-\beta_{0}\right)+o_{p}(1) \\
& \stackrel{p}{\longrightarrow} \tau_{*}^{\beta}\left(1-\tau_{*}^{\beta}\right) \int_{\mathbb{R}^{d}} \partial_{\beta} b\left(x, \beta_{0}\right)^{\top} A^{-1}\left(x, \alpha^{*}\right) \partial_{\beta} b\left(x, \beta_{0}\right) \mathrm{d} \mu_{\beta_{0}}(x)\left(d_{1}-d_{2}\right),
\end{aligned}
$$

and

$$
\begin{aligned}
& \left(1-\frac{\left[n \tau_{*}^{\beta}\right]}{n}\right)^{2} \frac{1}{\left(T \vartheta_{\beta}\right)^{2}} \sum_{i=1}^{\left[n \tau_{*}^{\beta}\right]} \mathbb{E}_{\beta_{1}^{*}}\left[\zeta_{i}^{2} \mid \mathscr{G}_{i-1}^{n}\right]-\left(\frac{\left[n \tau_{*}^{\beta}\right]}{n}\right)^{2} \frac{1}{\left(T \vartheta_{\beta}\right)^{2}} \sum_{i=\left[n \tau^{*}\right]+1}^{n} \mathbb{E}_{\beta_{2}^{*}}\left[\zeta_{i}^{2} \mid \mathscr{G}_{i-1}^{n}\right] \\
& =\frac{1}{T \vartheta_{\beta}^{2}}\left(\left(1-\frac{\left[n \tau_{*}^{\beta}\right]}{n}\right)^{2} \frac{1}{T} \sum_{i=1}^{\left[n \tau_{*}^{\beta}\right]} \mathbb{E}_{\beta_{1}^{*}}\left[\zeta_{i}^{2} \mid \mathscr{G}_{i-1}^{n}\right]-\left(\frac{\left[n \tau_{*}^{\beta}\right]}{n}\right)^{2} \frac{1}{T} \sum_{i=\left[n \tau^{*}\right]+1}^{n} \mathbb{E}_{\beta_{2}^{*}}\left[\zeta_{i}^{2} \mid \mathscr{G}_{i-1}^{n}\right]\right) \\
& \stackrel{p}{\longrightarrow} 0 .
\end{aligned}
$$

Therefore, from Lemma 9 of Genon-Catalot and Jacod (1993), we see

$$
\frac{1}{T \vartheta_{\beta}} \sum_{i=1}^{\left[n \tau_{*}^{\beta}\right]} \zeta_{i}-\frac{\left[n \tau_{*}^{\beta}\right]}{n} \frac{1}{T \vartheta_{\beta}} \sum_{i=1}^{n} \zeta_{i} \stackrel{p}{\longrightarrow} \tau_{*}^{\beta}\left(1-\tau_{*}^{\beta}\right) \int_{\mathbb{R}^{d}} \partial_{\beta} b\left(x, \beta_{0}\right)^{\top} A^{-1}\left(x, \alpha^{*}\right) \partial_{\beta} b\left(x, \beta_{0}\right) \mathrm{d} \mu_{\beta_{0}}(x)\left(d_{1}-d_{2}\right)
$$

Hence, from (7.57) and this, we obtain

$$
\frac{1}{T \vartheta_{\beta}} \sum_{i=1}^{\left[n \tau_{*}^{\beta}\right]} \hat{\zeta}_{i}-\frac{\left[n \tau_{*}^{\beta}\right]}{n} \frac{1}{T \vartheta_{\beta}} \sum_{i=1}^{n} \hat{\zeta}_{i} \stackrel{p}{\longrightarrow} \tau_{*}^{\beta}\left(1-\tau_{*}^{\beta}\right) \int_{\mathbb{R}^{d}} \partial_{\beta} b\left(x, \beta_{0}\right)^{\top} A^{-1}\left(x, \alpha^{*}\right) \partial_{\beta} b\left(x, \beta_{0}\right) \mathrm{d} \mu_{\beta_{0}}(x)\left(d_{1}-d_{2}\right) \neq 0,
$$


which implies $\mathcal{T}_{2, n}^{\beta} \longrightarrow \infty$ from $\left(7.56\right.$ and $T \vartheta_{\beta}^{2} \longrightarrow \infty$, that is, $P\left(\mathcal{T}_{2, n}^{\beta}>w_{q}(\epsilon)\right) \longrightarrow 1$

\section{ACKNOWLEDGEMENTS}

This work was partially supported by JST CREST Grant Number JPMJCR14D7 and JSPS KAKENHI Grant Number JP17H01100.

\section{REFERENCES}

Csörgö, M., Horváth, L. (1997). Limit Theorems in Change-Point Analysis, Wiley, New York.

De Gregorio, A., Iacus, S. M. (2008). Least squares volatility change point estimation for partially observed diffusion processes. Communications in Statistics - Theory and Methods, 37, 2342-2357.

Genon-Catalot, V., Jacod, J. (1993). On the estimation of the diffusion coefficient for multidimensional diffusion processes. Annales de l'Institut Henri Poincaré Probabilités et Statistiques, 29, 119-151.

Hall, P., Heyde, C. C. (1980). Martingale limit theory and its application. Academic Press, New York.

Iacus, S. M., Yoshida, N. (2012). Estimation for the change point of volatility in a stochastic differential equation. Stochastic Processes and their Applications, 122, 1068-1092.

Kessler, M. (1995). Estimation des paramètres d'une diffusion par des contrastes corrigés. Comptes rendus de l'Académie des sciences. Paris Serie I 320, 359-362.

Kessler, M. (1997). Estimation of an Ergodic Diffusion from Discrete Observations. Scandinavian Journal of Statistics, 24, 211-229.

Lee, S. (2011). Change point test for dispersion parameter based on discretely observed sample from SDE models. Bulletin of the Korean Mathematical Society, 48, 839-845.

McLeish, D., L. (1974). Dependent central limit theorems and invariance principles. The Annals of Probability, 2, $620-628$.

Negri, I., Nishiyama, Y. (2017). Z-process method for change point problems with applications to discretely observed diffusion processes. Statistical Methods and Applications, 26, 231-250.

Song, J. (2020). Robust test for dispersion parameter change in discretely observed diffusion processes. Computational Statistics and Data Analysis, 142, 106832.

Song, J., Lee, S. (2009). Test for parameter change in discretely observed diffusion processes. Statistical Inference for Stochastic Processes, 12, 165-183.

Tonaki, Y., Kaino, Y., Uchida, M. (2020). Adaptive tests for parameter changes in ergodic diffusion processes from discrete observations. arXiv:2004.13998

Uchida, M., Yoshida, N. (2011). Estimation for misspecified ergodic diffusion processes from discrete observations. European Series in Applied and Industrial Mathematics: Probability and Statistics, Volume 15, 270-290.

Uchida, M., Yoshida, N. (2012). Adaptive estimation of an ergodic diffusion process based on sampled data. Stochastic Processes and their Applications, 122, 2885-2924. 